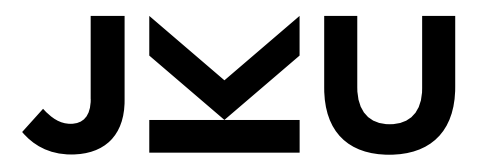

JOHANNES KEPLER UNIVERSITÄT LINZ

Eingereicht von

MMag. Dr. Markus Gole

Angefertigt am

Institut für Philosophie und

Wissenschaftstheorie

Betreuer / Betreuerin und

Erstbeurteiler / Erstbeurteilerin

Emeritus Dr. Volker

Gadenne

\title{
Der Physikalismus als Lösung des Bewusstseinsproblems
}

Zweitbeurteiler/

Zweitbeurteilerin

Ao. Univ.-Prof. i. R. Dr.

Johann C. Marek

Juli 2021

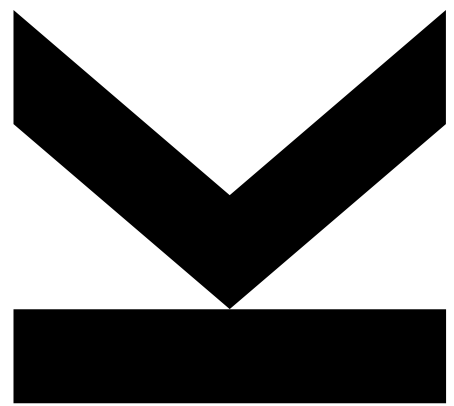

Dissertation

zur Erlangung des akademischen Grades

Doktor der Geistes- und Kulturwissenschaften

im Doktoratsstudium

Geistes- und Kulturwissenschaften 


\section{EIDESSTATTLICHE ERKLÄRUNG}

Ich erkläre an Eides statt, dass ich die vorliegende Dissertation selbstständig und ohne fremde Hilfe verfasst, andere als die angegebenen Quellen und Hilfsmittel nicht benutzt bzw. die wörtlich oder sinngemäß entnommenen Stellen als solche kenntlich gemacht habe.

Die vorliegende Dissertation ist mit dem elektronisch übermittelten Textdokument identisch.

Linz, 13.07.2021

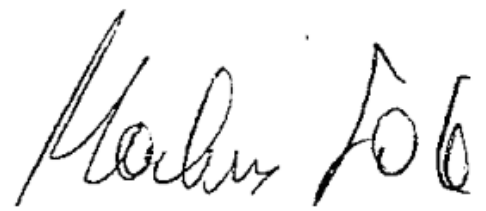

Markus Gole 


\section{Vorbemerkung Sprachgebrauch}

Die Lektüre wissenschaftlicher Texte an sich ist bereits eine Herausforderung, zwar genüsslich und genugtuend, aber nichtsdestotrotz kognitiv fordernd. Um zur maximalen Einfachheit des Textes im Sinne der Lesbarkeit und des Leseflusses beizutragen, wurde bewusst auf ein Gendern verzichtet. Dieser Verzicht bedeutet nicht, dass es keine Philosophinnen, Autorinnen, Dualistinnen oder Physikalistinnen gibt, oder wenn es sie gibt, dass sie gar unwichtig seien. Es bedeutet vielmehr, dass die Lektüre für jedweden Lesenden insofern aufbereitet wurde, dass durch ein Vermeiden unnötig komplexer Formulierungen und Stilblüten einer gendersensiblen deutschen Sprache der Fokus ausschließlich auf den Inhalt gelegt werden kann.

Selbstverständlich ist bei jedem männlichen Ausdruck auch das weibliche Geschlecht mitgemeint, ohne auch nur in irgendeiner Weise das Weibliche zu schmälern, zu vergessen, unter den Teppich zu kehren oder zu ignorieren. 


\section{Danksagung}

Ich möchte mich vor allem bei Volker Gadenne für die Betreuung dieser Arbeit bedanken.

Philosophie muss zum Denken und Diskutieren anstoßen; das geschieht wohl am besten, wenn nicht alle einer Meinung sind. Volker Gadenne und ich liegen sehr weit mit unseren philosophischen Überzeugungen auseinander, dennoch waren unsere Diskussionen von Wohlwollen, Scharfsinn und gegenseitigem Verständnis geprägt. Ich hätte mir keine bessere Unterstützung und kein freieres Arbeiten wünschen können. Es ist mir auch eine große Freude, Johann Marek als weiteren Beurteiler dieser Arbeit gewonnen zu haben. Mit Anfang 20 belegte ich die ersten Kurse zur Philosophie des Geistes bei ihm. Nun, fast 20 Jahre später, begleitet er mich durch meine Promotion. Niemand kennt meine ersten Schritte am Parkett der Philosophie des Geistes so gut wie er, genauso wie meine Entwicklungen, die ich hoffentlich eher mehr als weniger seit unserem ersten Zusammentreffen durchlaufen habe. 


\section{Kurzzusammenfassung (Deutsch)}

Wir wissen, wie es ist, Schmerzen zu haben, jemanden zu lieben oder Angst zu haben. Diese subjektiven Erlebnisqualitäten werden "Qualia" (Singular: Quale) genannt und die Frage nach deren Platz in einer objektiven, naturwissenschaftlich geprägten Welt stellt das

Bewusstseinsproblem dar. Eine Lösung ist die These des Physikalismus, die behauptet, dass alles Existierende physikalisch sei. Das Ziel der vorliegenden Arbeit ist es, die These des Physikalismus zu explizieren und zu zeigen, dass die hier erarbeitete Version auch wahr ist. Ich beginne damit, den Begriff "physikalisch" zu definieren, um daraufhin vorhandene Spielarten des Physikalismus darzulegen. Dabei werden bisherige ungelöste Probleme der zeitgenössischen Debatte gelöst, um daraus eine gangbare These des Physikalismus zu entwickeln. Die hier zu verteidigende Spielart des Physikalismus wird als eine Typen-Identitätsthese a priori mit disjunktiver Erweiterung um den Eliminativismus verstanden. Das bedeutet, dass entweder die Identität zwischen mentalen Entitäten und physikalischen Entitäten ohne Rückgriff auf Erfahrung aus der Summe aller naturwissenschaftlichen Erkenntnisse abgeleitet werden kann oder mentale Entitäten von der ontologischen Bildfläche vollständig eliminiert werden können. Schließlich wird durch ein neues Argument gezeigt, welches ich als das generalistische Argument für den Physikalismus bezeichne, dass die Wahrheit des Physikalismus deduktiv aus dessen Prämissen folgt. Der Physikalismus setzt sich somit innerhalb der Philosophie des Geistes als die überzeugendste Lösung des Bewusstseinsproblems durch. 


\section{Abstract (English)}

We know what it is like to be in pain, to love someone, or to be anxious. These subjective experiences are so-called "qualia" (in its singular form: "quale") and the question of how qualia fit into our world being described by the standards of objective natural sciences, constitutes the problem of consciousness. One solution to this problem is the thesis of physicalism which states that every existing thing is physical. The two goals of the present work are as follows. First, I make explicit to what the physicalistic thesis amounts to and second, how that version of physicalism turns out to be true. I start out by defining the concept of the physical and afterwards, I introduce different options of how the thesis of physicalism might be fleshed out further. In doing so, I solve previously unsolved problems in the current debate which ultimately sets the stage for the development of a kind of physicalism deserving the name and being worthy of defending. The thesis of physicalism, as I develop it here, is an a priori type identity thesis as the first disjunct paired together with eliminativism as the second disjunct. That is, either I know with justification independent of experience that all mental entities are identical with physical entities, for the identity claim can be derived from all the physical facts I know beforehand, or all mental entities can simply be eliminated from our ontology, without any remainder. Finally, I introduce a new argument for physicalism which I call generalized argument for physicalism. It shows that the truth of physicalism follows deductively from a set of premises. Thus, at the end of the day, physicalism stands its ground as the most compelling solution to the problem of consciousness. 


\section{DER PHYSIKALISMUS ALS LÖSUNG DES BEWUSSTSEINSPROBLEMS}

\section{Inhaltsverzeichnis}

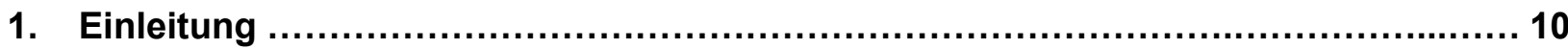

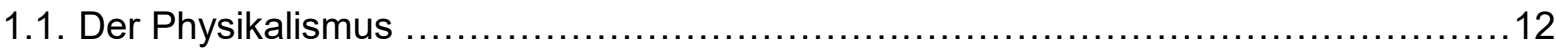

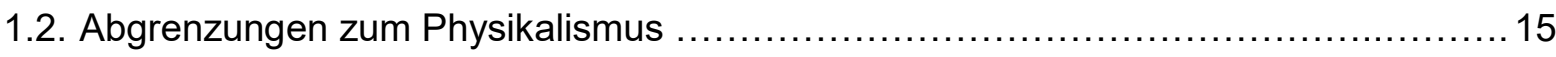

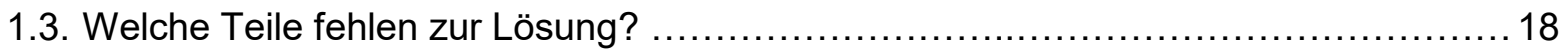

Erster Exkurs E1: Die Intentionalität der Gemütsbewegungen bei Brentano ................ 22

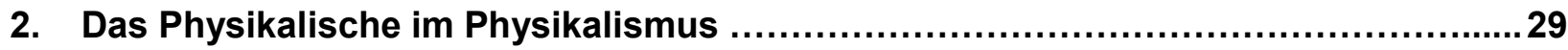

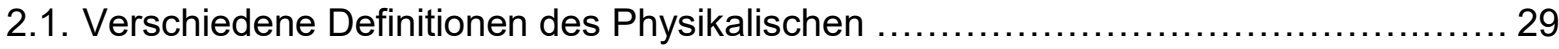

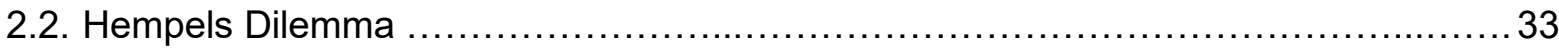

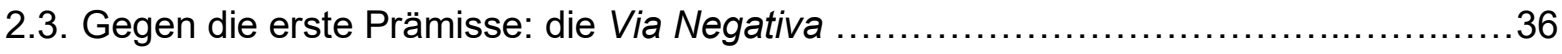

2.4. Das erneute Auftauchen von Hempels Dilemma ....................................... 40

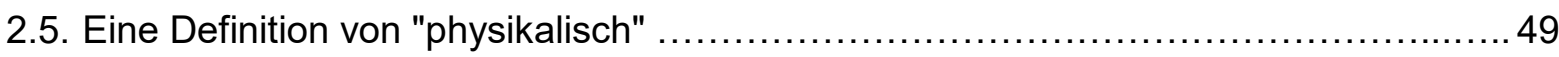

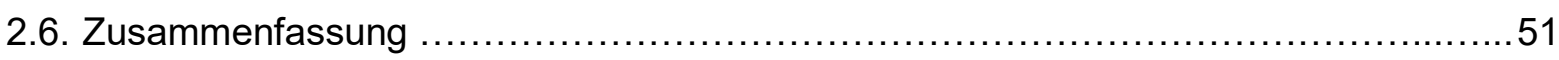

Zweiter Exkurs E2: Die Via Negativa und schwacher Mentalismus ........................... 53

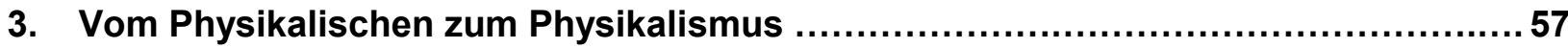

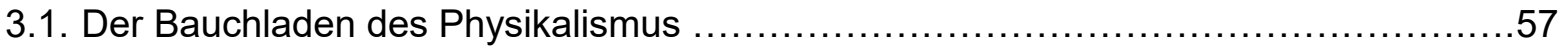

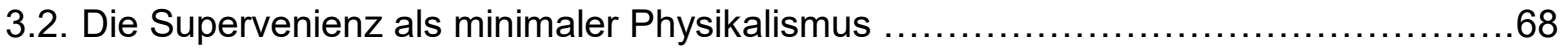

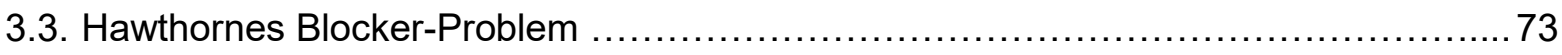

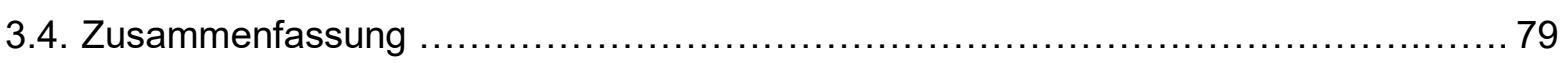

Dritter Exkurs E3: Eine Symmetrie-Relation zwischen möglichen Welten ................... 81

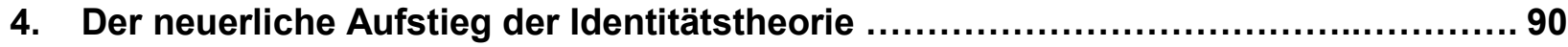

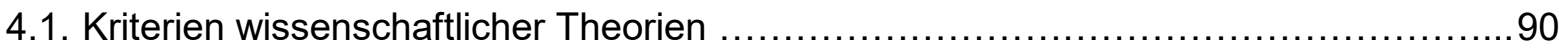

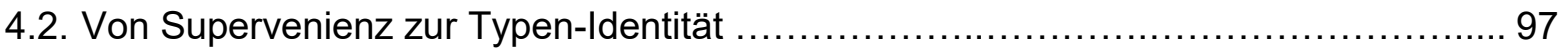

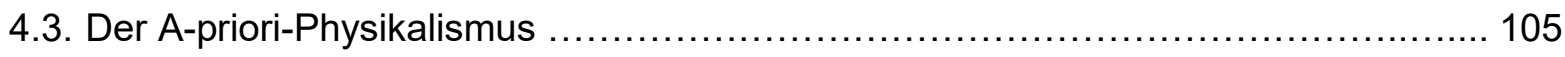

4.4. Der Physikalismus als Sprachprogramm ............................................... 110

4.5. Das Mentale und Physikalische: eine Definition deren Relation Teil 1 .................119 


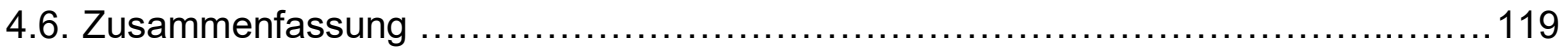

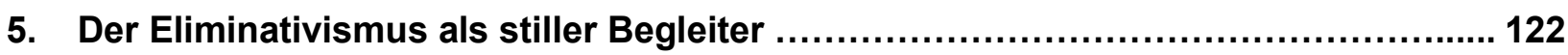

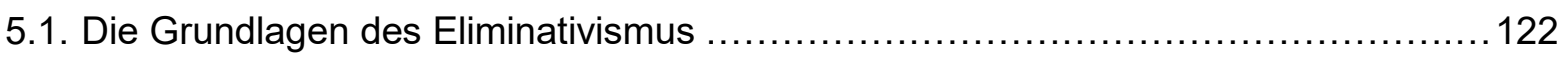

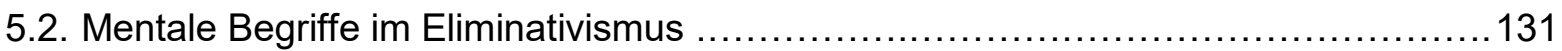

5.3. Wie einfach und sparsam ist der liberale A-priori-Eliminativismus? ................. 138

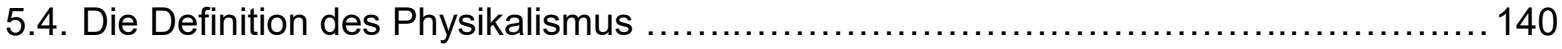

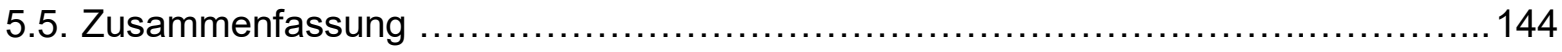

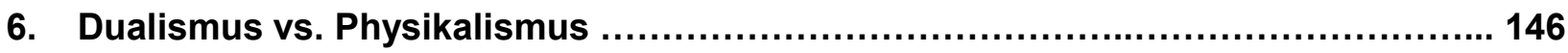

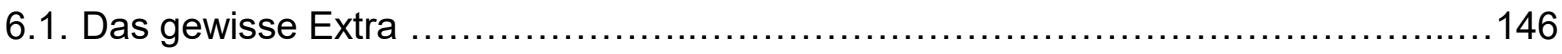

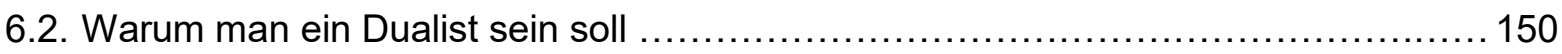

6.3. Warum man ein Physikalist sein soll ................................................ 156

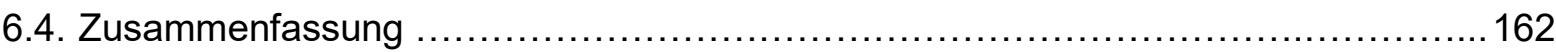

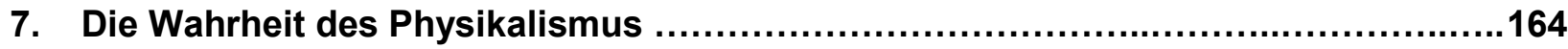

7.1. Das generalistische Argument für den Physikalismus .............................. 164

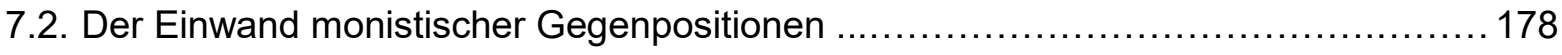

7.3. Warum der physikalische Monismus gesichert ist ............................... 181

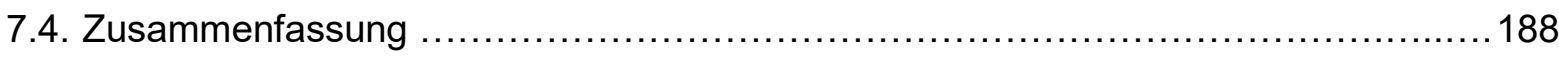

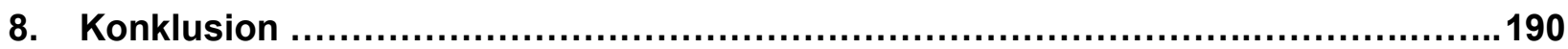

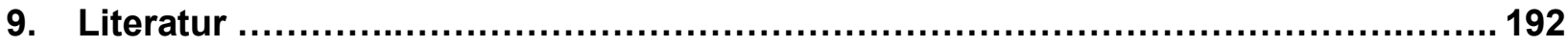




\section{Well}

\section{What's the story, morning glory?}

Oasis (1995) 


\section{Einleitung}

In der Philosophie des Geistes nimmt das Leib-Seele-Problem eine zentrale Rolle ein. Dieses Problem besteht darin, anzugeben, in welcher Art und Weise etwas Mentales (Seele) mit der physikalischen Welt (Leib) in Verbindung steht. In der gegenwärtigen Diskussion wird das LeibSeele-Problem gerne in der Formulierung des Qualia-Problems gebracht. In einem intuitiven Verständnis wird mit einem Quale (Plural: Qualia) die subjektive Erlebnisqualität, eine phänomenale Eigenschaft, bezeichnet. Der Begriff "Quale" wurde Anfang des 20. Jahrhunderts von Lewis eingeführt und lässt sich auf folgende Textstelle zurückverfolgen (Lewis, 1929, S. 121):

There are recognizable qualitative characters of the given, which may be repeated in different experiences, and are thus a sort of universals; I call these "qualia". But although such qualia are universals, in the sense of being recognized from one to another experience, they must be distinguished from the properties of objects.

Aus diesem Zitat geht hervor, dass das Gegebene eine qualitative Eigenschaft besitzt - ein Quale - und verschieden zu Eigenschaften von Objekten ist, wie beispielsweise die Farbe Rot einer Rosenblüte. In der aktuellen Diskussion in der Philosophie des Geistes hat sich an diesem Grundverständnis von Qualia nichts Gravierendes verändert. Mit einem Quale wird die subjektive Erlebnisqualität einer Rotwahrnehmung gemeint, wenn ich eine reife Tomate betrachte oder auch die subjektive Erlebnisqualität des Schmerzes, wenn ich mich beim Rasieren in die Wange schneide. Ein Quale ist das Wie-es-Ist eines bewussten Erlebnisses (Nagel, 1974) $)^{1}$.

Qualia stellen nun deswegen ein Problem dar, weil es keinen Konsens hinsichtlich deren ontologischen Status gibt. Das Lager der Physikalisten meint, dass Qualia nichts anderes als physikalische Eigenschaften des Gehirns sind (z.B. Smart, 1959), und das Lager der Dualisten hingegen meint, dass Qualia fundamental mentale Eigenschaften des physikalischen Gehirns (z.B. Chalmers, 1996) bzw. fundamental mentale Eigenschaften einer immateriellen Seele sind (z.B. Swinburne, 1986). Eliminativisten gehen sogar noch einen Schritt weiter und argumentieren, dass Qualia bloße Illusion sind (z.B. Rorty, 1965). Zwischen diesen verschiedenen typischen Positionen innerhalb der Philosophie des Geistes gibt es wiederum verschiedene Nuancen und Schattierungen. Die Antwort auf die Frage nach der Existenz von Qualia und das Verhältnis von Qualia zu physikalischen Eigenschaften ist ausschlaggebend

\footnotetext{
1 Siehe dazu auch Farrell (1950, S. 183), der diese Phrase als Erstes gebraucht hat und bereits die wesentliche Frage von Nagel vorweggenommen hat: "I wonder what it would be like to be, and hear like, a bat". Darüber hinaus wird in der aktuellen Literatur immer noch die genaue Bedeutung dieser kanonischen Phrase diskutiert (Stoljar, 2016). 
dafür, welche Bewusstseinstheorie letztendlich vertreten wird. Das Finden dieser Antwort stellt das Qualia-Problem bzw. das philosophische Bewusstseinsproblem dar.

In der gegenwärtigen Psychologie und den Neurowissenschaften wird im Großen und Ganzen nur vage Stellung genommen zum ontologischen Status vermeintlich mentaler Eigenschaften. Hier wird die Diskussion nicht im Vokabular des Qualia-Problems geführt, sondern vor allem im Vokabular der Emotionswissenschaften. Dabei verwendet die Psychologie als Wissenschaft des Verhaltens und Erlebens einen begrifflichen Dualismus. Damit ist gemeint, dass neben der physikalischen Sprechweise, wie beispielsweise das Feuern von Neuronen in gewissen Gehirnarealen, ebenfalls eine mentale Sprechweise Anwendung findet, wie beispielsweise die über Schmerzen. Hier ist wichtig, herauszuheben, dass die Frage nach der konkreten Abhängigkeitsrelation (z.B. die Relation einer Identität) von Schmerzen und einem Neuronenfeuern in ontologischer Hinsicht unbeantwortet bleibt und durch einen begrifflichen Dualismus weder bejaht noch verneint wird. Gleichzeitig geht mit einem begrifflichen Dualismus in der Psychologie auch eine andere Art und Weise, wie Wissen über diese Gebiete gesammelt wird, einher. So zieht eine mentale Sprechweise teilweise einen Rückgriff auf Daten aus der Erste-Person-Perspektive nach sich und eine physikalische Sprechweise einen Rückgriff auf Daten aus der Dritte-Person-Perspektive. Dieser Sachverhalt lässt sich wie folgt näher beschreiben.

Um herauszufinden, wie intensiv Schmerzen von den jeweiligen Personen empfunden werden, kommt beispielsweise ein Fragebogen oder auch eine Methode aus den Neurowissenschaften zum Einsatz. Dadurch wird versucht, die erlebte Schmerzintensität so weit wie möglich objektiv messbar zu machen. In diesem Sinne kann das psychologische Bewusstseinsproblem formuliert werden: Es besteht im geeigneten Messbarmachen und objektiven Erfassen von mentalen Eigenschaften. Objektivität bezieht sich in diesem Sinne nur darauf, dass unabhängig von der Person, die als Versuchsleiter fungiert und die Daten auswertet, zum selben Ergebnis gelangt wird. Es ist nicht objektiv im Sinne eines Auskommens ohne Rückgriff auf Introspektion aufseiten des Befragten. Die empfundene Schmerzintensität wird indirekt erhoben und beispielsweise als ein bestimmter Punktewert in einem Fragebogen messbar gemacht bzw. operationalisiert, oder auch als eine Gehirnaktivität während einer Schmerzapplikation im Vergleich zu einer Kontrollbedingung erhoben und dergleichen. Ob nun aber das messbar Gemachte (Punktewert im Fragebogen) dasselbe ist wie das zu messen Wollende (empfundene Schmerzintensität) selbst, bedarf eines weiteren Arguments, welches jemanden entweder in das physikalistische oder dualistische Lager einordnet. Die Psychologie macht oftmals von Methoden Gebrauch, die ebenfalls die Erste-Person-Perspektive des jeweiligen Subjekts miteinbeziehen. Denn ohne eine vorige introspektive Erfassung der eigenen mentalen Vorgänge gibt es im Zuge der Erkenntnisgewinnung in der Psychologie nichts objektiv Messbares. Im Vergleich dazu benötigt das Messen der Halbwertszeit eines radioaktiven Stoffes ausschließlich Methoden aus der Dritte-Person-Perspektive, ohne jemals im Zuge der 
Erkenntnisgewinnung auf Methoden aus der Erste-Person-Perspektive des radioaktiven Stoffes selbst angewiesen zu sein.

In diesem Sinne ist ein Rekurs auf subjektive Erlebnisse ebenfalls im psychopathologischen Bereich zu finden. Die aktuelle Version des Diagnostic and Statistical Manual of Mental Disorders (DSM-V, American Psychiatric Association, 2013) führt verschiedenste mentale Faktoren als die Kardinalsymptome vieler psychischer Störungen an. Beispiele hierfür sind eine depressive Verstimmung für unipolare affektive Störungen und eine Furcht vor spezifischen Dingen bei bestimmten Angststörungen. Des Weiteren werden auch diese mentalen Faktoren in der Psychotherapie in den Mittelpunkt gerückt und zu verändern versucht, was interessanterweise mit spezifischen neuroplastischen Veränderungen im Gehirn einhergeht (siehe für eine Übersicht Beauregard, 2007). Dieser Punkt ist deswegen philosophisch interessant, weil - methodisch - etwas Mentales zu verändern versucht wird und gleichzeitig auch etwas Physikalisches verändert wird. Dies entspricht unserer Intuition von kausal wirksamen mentalen Eigenschaften. Aber gleichzeitig wird dadurch das Problem der mentalen Kausalität heraufbeschworen, falls eine (ontologisch-)dualistische Position vertreten wird und gleichzeitig die kausale Geschlossenheit des Physikalischen angenommen wird. Die kausale Geschlossenheit des Physikalischen besagt, dass alle Wirkungen in unserer physikalischen Welt hinreichend durch physikalische Ursachen erklärt werden können. Das Problem der mentalen Kausalität besteht im Wesentlichen nun darin, dass etwas Wesensverschiedenes zu etwas Physikalischem (hier: etwas Mentales) tatsächlich auch Veränderungen in unserer physikalischen Welt veranlasst. Hier gibt es nun den ersten Anknüpfungspunkt zwischen der Psychologie und der Philosophie. Es ist einerseits das Erkennen einer Unvereinbarkeit zwischen unseren alltäglichen Erfahrungen unseres kausal wirksamen und außerordentlich besonderen mentalen Lebens und dem bis jetzt vorhandenen und anerkannten Wissen, wie unser nicht-mentales Lebens funktioniert. Denn unser nichtmentales Leben kommt ohne Mentalität aus, aber unser mentales Leben hängt mit physikalischen, biologischen, chemischen und allen weiteren naturwissenschaftlichen Gegebenheiten zusammen. Andererseits fehlen klare und einleuchtende Theorien, seien es naturwissenschaftliche oder philosophische, die das gemeinsame Wirken von zwei völlig wesensverschiedenen Dingen erklären.

\subsection{Der Physikalismus}

Es gibt viele verschiedene Charakterisierungen des Physikalismus. Intuitiv besagt der Physikalismus, dass alles in unserer Welt physikalisch sei. Es gibt nichts anderes als genau das Physikalische und Materielle und nichts darüber hinaus hat einen Platz in unserer Welt. Alles Existierende sei schlichtweg nichts anderes als etwas Physikalisches. Im Rahmen dieser 
vorliegenden Arbeit verwende ich "physikalisch" in einem weitgefassten Sinne, um mich damit auch auf biologische, chemische und alle anderen Wahrheiten aus den Naturwissenschaften zu beziehen. Gleichsam verwende ich "Physik" als einen Begriff, der sich auf die Naturwissenschaft als Gesamtes bezieht und somit auch Disziplinen wie beispielsweise Biologie und Chemie umfasst. Dieses Vorgehen soll im Wesentlichen einer besseren Lesbarkeit und Übersichtlichkeit dienen. Dem gegenüber stehen Positionen, die nicht-physikalische Entitäten wie mentale Substanzen oder mentale Eigenschaften in ihrer Ontologie zulassen. Diese dualistischen Positionen erlauben somit zwei ontologische Kategorien, wobei in einer Kategorie alles Physikalische und Materielle zu finden ist sowie in einer anderen Kategorie alles Mentale und Geistige zu finden ist. Es hat sich auch in der gegenwärtigen Literatur eingebürgert, dass von mentalen und physikalischen Eigenschaften die Rede ist. Hier kann in der vorliegenden Arbeit getrost der Begriff der Eigenschaften durch Begriffe wie Vorgänge, Sachverhalte, Vorkommnisse, Dinge, Entitäten und dergleichen ausgetauscht werden, denn es hängt nichts Wesentliches an diesen Ausdrücken. Zur Einheitlichkeit werde ich großteils den Begriff der Entität verwenden und damit Eigenschaften, Vorgänge, Sachverhalte, Vorkommnisse, Dinge und dergleichen mitmeinen.

Die ursprüngliche Motivation, solch eine gewagte These des Physikalismus vorzulegen, ist es, eine überschaubare, sparsame, vollständige und richtige Beschreibung unserer Welt zu liefern, in der sowohl Entitäten ohne Bewusstsein als auch Entitäten mit Bewusstsein auftauchen. Der Physikalismus ist somit eine These in der Philosophie des Geistes mit dem vorrangigen Ziel, eine befriedigende Erklärung zu geben, wie und warum das Bewusstsein, und ebenso alles andere Mentale, in einer wissenschaftlichen und physikalistischen Welt seinen Platz findet. Das physikalistische Unterfangen ist es somit, dass am Ende des Tages dem vermeintlich wesensverschiedenen Bewusstsein sein angestammter Platz in einer physikalistischen Welt zugewiesen wird. Viele Argumentationen für den Physikalismus sind induktiver Natur und beziehen sich auf den immensen Fortschritt der Naturwissenschaften und insbesondere den voranschreitenden Neurowissenschaften. Eine Verfechterin einer physikalistischen Lösung des Körper-Geist-Problems auf Basis der ständig erweiternden Naturwissenschaften ist P. S. Churchland. So fasst sie diesen Punkt treffend zusammen (P. S. Churchland, 2008, S. 409):

The history of science can be seen as a gradual process whereby speculative philosophy cedes intellectual space to increasingly well-grounded experimental disciplines - first astronomy, but followed by physics, chemistry, geology, biology, archaeology, and more recently, ethology, psychology, and neuroscience. Science now encompasses plausible theories in many domains, including large-scale theories about the cosmos, life, matter, and energy. The mind's turn has now come. 
Dies hängt natürlich auch mit den immensen Datenmengen zusammen, die neuere Forschungsmethoden der Neurowissenschaften generieren, begonnen bei der Einzelzellableitung bis hin zur funktionellen Magnetresonanz (siehe v.a. Graphik 1 in Sejnowski et al., 2014). Diese stetig wachsenden Erkenntnisse vermögen noch mehr Einsichten in das klassische philosophische Bewusstseinsproblem zu bringen.

Die Tatsache, dass mentale Vorgänge mit Vorgängen im Gehirn zusammenhängen, ist unumstritten und wird sowohl von Dualisten als auch Physikalisten akzeptiert. In den letzten Dekaden hat sich das Wissen um die neuronalen Korrelate des Bewusstseins rapide angehäuft. Verschiedene Fragen auf diesem Gebiete sind begrifflicher, methodischer und ontologischer Natur (Chalmers, 2000). Es ist eine begriffliche Frage, was man unter einem neuronalen Korrelat des Bewusstseins versteht, eine methodische Frage, wie man diese neuronalen Korrelate am besten identifiziert, und schließlich eine ontologische Frage, ob die beiden korrelierten Vorgänge im Grunde ein und derselbe Vorgang sind. So wurde das neuronale Korrelat des Bewusstseins als die minimale Menge an neuronaler Aktivität und beteiligter Strukturen definiert, die für ein bewusstes Erleben hinreichend sind (Crick \& Koch, 1990). Eine Möglichkeit zur Identifizierung der neuronalen Korrelate stellen bildgebende Verfahren in den Neurowissenschaften dar (v.a. die funktionelle Magnetresonanztomographie) und eine Forschungsrichtung ist die der affektiven Neurowissenschaften, bei der unter anderem nach den neurobiologischen Grundlagen des Emotionserlebens gesucht wird. Eine wichtige Frage ist, welche ontologischen Aussagen über Qualia, ausgehend von diesen naturwissenschaftlichen Aussagen, getroffen werden können. Es scheint beinahe so zu sein, dass es in den Naturwissenschaften eher verpönt ist, sich auf solche metaphysischen - rein philosophischen Diskussionen einzulassen und nach der Natur von mentalen Eigenschaften zu fragen. Glücklicherweise haben Naturwissenschaftler begonnen, genau diese Fragen zu stellen, ersichtlich in den steigenden Publikationen in naturwissenschaftlichen Journalen. Denn jedes Forschungsergebnis muss in geeigneter Weise interpretiert und in das vorhandene Wissen eingeordnet werden.

So finden sich hie und da auch explizit Diskussionen zum ontologischen Status von mentalen Eigenschaften. Barrett et al. (2007) sehen Qualia als emergente Phänomene an, die aus der gleichzeitigen Aktivität verschiedener präfrontaler und limbischer Areale entstehen. Sie übernehmen explizit Searles Biologischen Naturalismus, ohne sich jedoch seiner idiosynkratischen Sichtweise näher zu widmen. Searle (z.B. 2002) versteht unter seinem Biologischen Naturalismus lediglich, dass Qualia kausal auf neurobiologische Prozesse zurückführbar sind, mentale Eigenschaften aber nicht ontologisch darauf reduzierbar sind. Im selben Atemzug wird behauptet, dass damit kein Eigenschaftsdualismus hergestellt ist, sondern Qualia ein Teil unserer physikalischen Wirklichkeit sind. Zudem spricht er sich explizit gegen die klassische Trennung von objektiven und subjektiven Dingen aus, was physikalische und mentale 
Dinge in verschiedene, einander ausschließende, Kategorien gibt. Zu guter Letzt wehrt sich Searle dagegen, dass seine Position als nicht-reduktiver Physikalismus aufgefasst wird, nämlich weder im Sinne einer Supervenienz noch einer Emergenz. Unter einer Supervenienz versteht man, dass jede Änderung im mentalen Bereich auch mit einer Änderung im physikalischen Bereich einhergeht. Wenn also beispielsweise ein Schmerz-Quale durch ein Angst-Quale abgelöst wird, und eine bestimmte Gehirnaktivität die Supervenienzbasis darstellt, dann hat sich auch die zugrundeliegende Gehirnaktivität verändert. Hingegen bedeutet eine Emergenz von Qualia, dass diese aus bestimmten Gehirnaktivitäten plötzlich hervortreten und so auf der ontologischen Bildfläche erscheinen. Dabei sind emergente Qualia nicht auf die Emergenzbasis reduzierbar, sondern sind etwas komplett Neues. In ähnlicher Weise wird von psychiatrischer Seite dezidiert eine Art nicht-reduktiver Materialismus angenommen (Kendler, 2005), ohne jedoch diese Position näher zu beleuchten und sich deren Probleme bewusst zu sein. Wieder andere bekennen sich offen zur genuin mentalen Natur der Qualia, die aus der zugrunde liegenden neuronalen Aktivität emergieren (Libet, 2006). Rätselhaft bleibt bei all diesen Ansätzen, was genau darunter verstanden wird, denn ein zu Erklärendes durch etwas zu erklären, das selbst nebulos bleibt, ist kein wirklicher Schritt voran.

Ein Autor, der sich zwischen den Welten der Naturwissenschaften und der Philosophie bewegt und dabei auch beiden Seiten gerecht werden will, ist Antonio Damasio. Nach Damasio (z.B. 1994) sind Qualia dasjenige, was vor allem von bestimmten somatosensorischen und limbischen Arealen repräsentiert wird. Damasio kann also guten Rechts als Repräsentationalist bezeichnet werden, da er Qualia im ersten Schritt mit dem intentionalen Gehalt dieser subjektiven Erlebnisinhalte gleichsetzt. Unter Intentionalität wird die Gerichtetheit von gewissen mentalen Zuständen verstanden. Im zweiten Schritt stellt er Repräsentationen als nichts anderes als gewisse Erregungsmuster im Gehirn dar. Er spricht sich gegen den Irrtum von Descartes aus, der Körper und Geist als zwei verschiedene Substanzen ansieht, und folgert daraus seinen Ansatz. Was Damasio aber nicht bringt, ist ein Argument für seine Position und ein Vergleich mit konkurrierenden Positionen. Er argumentiert nicht umsichtig genug für seinen Ansatz, denn aus der Falschheit eines cartesischen Substanzdualismus folgt nicht automatisch Damasios Repräsentationalismus. Hier zeigt sich interessanterweise, dass dieselben empirische Erkenntnisse von philosophisch arbeitenden Naturwissenschaftlern anderes interpretiert werden und auch hier Abschweifungen in dualistische Positionen geschehen.

\subsection{Abgrenzungen zum Physikalismus}

Ich möchte hier auch sagen, was der Physikalismus nicht ist. Er ist keine triviale Wahrheit, so wie es eine Tautologie darstellen würde. Falls der Physikalismus wahr ist, ist es eine nichttriviale Wahrheit, denn die Wahrheit hängt konkret davon ab, wie unsere aktuale Welt und hinreichend ähnliche Welten tatsächlich beschaffen sind (Jackson, 1998a; Lewis, 1994). Als 
Konsequenz ist der Physikalismus eine kontingente These über kontingente Entitäten. Diese These ist kontingent, weil der Physikalismus wahr oder falsch sein kann, wobei die Wahrmacher kontingente Entitäten wie Steine, Bäume und Menschen mit ihren vielschichtigen psychischen Vorgängen sind. An diesem Punkt wird klar, dass der Physikalismus auch keine These über nicht-kontingente Entitäten ist. Nicht-kontingente Entitäten sind notwendige Entitäten, zu denen typischerweise Zahlen, Mengen, Funktionen, Universalien und dergleichen zählen. Ob diese notwendigen abstrakten Entitäten auch tatsächlich existieren, ist wiederum eine andere Frage. Gleichsam sind viele weitere interessante Fragen mit der Existenz von Abstrakta verbunden und jeder Physikalist, aus welchem Grund auch immer, vermag die eine oder andere Frage aufzugreifen. Physikalisten sollten sich frei fühlen, dies auch zu tun, doch sie sollten sich nicht verpflichtet fühlen, es zu müssen. Der Grund dafür ist, dass der Physikalismus eine These für eine bestimmte Angelegenheit ist und Thesen zu abstrakten Entitäten eine andere Angelegenheit ist, die von der Wahrheit des Physikalismus abgekoppelt ist (Crane \& Mellor, 1990; Crook \& Gillett, 2001).

Vielleicht ändert sich, aus welchen Gründen auch immer, der Konsens unter Physikalisten, sodass die Frage der Abstrakta aufgegriffen werden muss. In diesem Fall möchte ich den folgenden Notfallplan hervorkehren und zeigen, warum eine bestimmte Haltung zu Abstrakta keine Relevanz für die Wahrheit des Physikalismus hat ${ }^{2}$. Quine (1948, S. 32) hat weichenstellend argumentiert, dass zu existieren bedeutet, der Wert einer gebundenen Variable zu sein: "To be is, purely and simply, to be the value of a bound variable". Sobald eine Variable durch einen Quantor gebunden ist, ist man ontologisch zur Existenz dessen verpflichtet, wofür die Variable steht. Um Quines Beispiel zu Primzahlen zu verwenden, sollte die Aussage "Es gibt eine Primzahl zwischen 1000 und 1010" wie folgt analysiert werden: "Es gibt ein X zwischen 1000 und 1010, das die Eigenschaft 'eine Primzahl zu sein' hat". Durch diese Paraphrasierung wird $X$ zu einer gebunden Variable des Existenzquantors und man verpflichtet sich auf die Existenz von Primzahlen im Allgemeinen und in diesem Beispiel auf die Existenz der konkreten Primzahl 1009. Hinsichtlich der Mathematik stand für Quine immer klar fest, dass sie bis über beide Ohren hinaus zur Existenz von Abstrakta verpflichtet ist. Interessanterweise verteidigt Quine in späteren Arbeiten den Physikalismus, obwohl sich Quine auch gleichzeitig auf die Existenz von Abstrakta wie Funktionen und Mengen verpflichtet (Quine, 1960). Der Hauptgrund ist der, dass die Mathematik für die Physik vorausgesetzt wird, denn ohne Mathematik kann die Physik nicht ihrer Arbeit nachgehen und a fortiori wäre auch kein Physikalismus möglich. In derselben Argumentationslinie ist Mathematik auch für den Dualismus eine Voraussetzung, denn ohne Mathematik gäbe es auch keinen Substanzdualismus, der für zwei separate Substanzen argumentiert, oder auch keinen Eigenschaftsdualismus, der für zwei separate Eigenschaften argumentiert. Somit reicht es unterm Strich nicht aus, den Physikalismus

2 Siehe Rabin (2020), der das Problem von Abstrakta für den Physikalismus aufzeigt und drei Lösungsvorschläge anbietet. 
widerlegt zu wissen auf Basis der Akzeptanz von Abstrakta, oder gar noch strikter auf Basis der Möglichkeit von Abstrakta.

Des Weiteren ist der Physikalismus nicht ausschließlich eine empirische These (Prelevic, 2017; Dove, 2018; Elpidorou \& Dove, 2018) oder eine bloße Haltung (van Fraassen, 2002; Ney, 2008). Diese alternativen Ansätze des Physikalismus sind interessant und sagen beide etwas Wahres. So stimme ich überein, dass ein Physikalist eine objektiv zugängliche Wirklichkeit annimmt, die mit empirischen Methoden zugänglich ist. Ebenso stimme ich überein, dass ein Physikalist auch eine gewisse Haltung zu den empirischen Erkenntnissen hegt. Obwohl die Wahrheit des Physikalismus abhängig davon ist, wie unsere Welt empirisch beschaffen ist, und ein Physikalist auch typischerweise eine empirisch-wissenschaftliche Haltung mitbringt, sind beide Punkte jedoch nicht hinreichend für eine adäquate Definition des Physikalismus. Wenn man sich ausschließlich auf ein Forschungsprogramm oder eine Haltung beschränkt, fehlt zusätzlich genau das, was auch in der Philosophie des Geistes interessant ist. Von Interesse ist nämlich die Natur der mentalen Entitäten, die im physikalistischen Lager etwas Physikalisches darstellt und im dualistischen Lager etwas genuin Mentales darstellt. Kontra Prevelic, Dove sowie Elpidorou und Dove ist eine empirische These nicht mit dem Physikalismus gleichzusetzen, weil die Frage nach der Natur der mentalen Entitäten nicht mit dem Bewähren irgendeiner empirischen These gleichzusetzen ist. Der Schluss von empirischen Tatsachen, wie die einer Korrelation von Empfindungen und Gehirnprozessen, zur ontologischen Reduktion, wie die einer Identität zwischen Empfindungen und Gehirnprozessen, bedarf mehr als einer bloßen empirischen Arbeit. Ich möchte auch gegen van Fraassen sowie Ney in ähnlicher Weise argumentieren. Eine Haltung zur empirischen Forschung als Gesamtes (siehe van Fraassen) oder eine Haltung, wie man zu ontologischen Verpflichtungen kommt (siehe Ney), ist nicht mit dem Physikalismus gleichzusetzen, weil auch hier die Frage nach der Natur der mentalen Entitäten nicht mit irgendeiner Haltung gleichzusetzen ist.

Schließlich ist der Physikalismus auch nicht nicht-reduktiv. Das bedeutet, dass es keinen Raum zwischen einer Reduktion des Mentalen auf das Physikalische oder einer Elimination des Mentalen einerseits und der Existenz des Mentalen als etwas Fundamentales andererseits gibt. Dabei schließe ich mich den Analysen von Kim (1989) an und stimme überein, dass ein Raum zwischen den beiden zuvor beschriebenen Endpolen ein Mythos ist. Entweder kollabiert ein vermeintlicher nicht-reduktiver Physikalismus bei näherer Betrachtung in eine reduktive physikalistische Position oder er wandelt sich in eine dualistische Position, in welcher konkreten Spielart auch immer. Falls der nicht-reduktive Physikalist darauf besteht, den Mythos des Zwischenraumes zu leben und auf halbem Wege beiden Positionen entgegen zu kommen, ohne dabei eine Forderung beider Lager aufzugeben, dann verliert er sich bestenfalls in Unverständlichkeit oder schlechtestenfalls gar in Widersprüchlichkeit.

Andere halten jedoch dagegen und sehen den Mythos eines nicht-reduktiven Physikalismus als ein Produkt fehlgeleiteter und antiquierter Hintergrundannahmen, die 
gemeinsam mit reduktiven physikalistischen Positionen verschwinden hätten sollen (D'oro et al., 2019). Wie ich den Ansatz von D'oro und Kollegen verstehe, sichern sie die Autonomie und Unabhängigkeit des Mentalen als etwas intrinsisch Mentales, indem sie eine Integration in die Methodik der Naturwissenschaften ausschließen, denn unser mentales Leben lässt sich nur durch geisteswissenschaftliche Methoden erfassen. Unter diesen geisteswissenschaftlichen Methoden fallen all jene Methoden, die nicht auf eine objektive, subjektunabhängige Erkenntnisgewinnung ausgerichtet sind, sondern stets in der Erkenntnisgewinnung an das Subjekt selbst gebunden sind. Nach D'oro und Kollegen haben die naturwissenschaftlichen und geisteswissenschaftlichen Methoden auch immer einen Gegenstand der Betrachtung und unser mentales Leben muss mit beiden Methoden betrachtet werden, um es vollständig zu erfassen. Damit ist der nicht-reduktive Teil gerettet. Um den physikalistischen Teil ihres Ansatzes zu retten, sprechen sie sich für eine liberale Sicht aus auf das, was als Teil unserer Natur zu gelten hat. Somit weiten sie das Verständnis des Physikalischen so weit aus, dass sie auch dem Mentalen einen Platz in der Natur als etwas intrinsisch Physikalisches sichern können. Ich denke nicht, dass D'oro und Kollegen erfolgreich sind, denn sie haben lediglich die Grenzen ins Unkenntliche verwischt. Es bleibt völlig unbeantwortet, was das Mentale im Gegensatz zum Physikalischen nun tatsächlich unterscheidet und wie etwas gleichzeitig intrinsisch mental und intrinsisch physikalisch sein kann. Um diese Probleme aufzulösen, so fürchte ich, müssen auch D'oro und Kollegen Kims ursprüngliche Konklusion akzeptieren und entweder in das Lager des Physikalisten oder des Dualisten wechseln.

\subsection{Welche Teile fehlen zur Lösung?}

Der Physikalismus ist die These, dass alles Existierende nichts anderes als etwas Physikalisches sei, und somit wird behauptet, dass alles physikalisch ist. Ein integraler Bestandteil dieser Definition ist der Ausdruck "etwas Physikalisches". Doch was bedeutet es, "physikalisch" zu sein und was genau macht ein Ding zu einem physikalischen Ding? Versuche, diese Frage und eng damit zusammenhängende Fragen zu klären, gibt es zahlreiche. So wurde beispielsweise mit einiger Überzeugungskraft argumentiert, dass eine Definition des Begriffes "physikalisch" und die darauf aufbauende Charakterisierung des Physikalismus ein hoffnungsloses Unterfangen sei (v.a. Crane \& Mellor, 1990) und in einem Dilemma mündet, das ursprünglich von Hempel $(1969,1980)$ vorgebracht wurde. Doch um letztendlich zu einer bedeutungsvollen Diskussion innerhalb der Philosophie des Geistes zu gelangen, ist eine klare Definition unumgänglich und stellt den ersten Teil einer Lösung dar. Es kann erst dann in eine bedeutungsvolle Diskussion in der Philosophie des Geistes eingetreten werden, sobald sich die beteiligten Lager über die Sache, worum es letztendlich geht, klar verständigen können. Der Weg zu dieser Lösung wird in Kapitel 2 erarbeitet und schließt mit dem Vorschlag, dass 
"physikalisch" durch einen Bezug auf eine vollendete Physik definiert werden soll. Dabei wird gegen Hempels Dilemma (Hempel, 1969, 1980) argumentiert, das den Bezug auf irgendeine Physik, sei es eine gegenwärtige oder zukünftige, als fehlgeleitet in Definitionsversuchen des Physikalismus zeigen will. Das Dilemma besteht darin, dass der Physikalismus im Lichte einer gegenwärtigen physikalischen Theorie falsch ist und im Lichte einer zukünftigen physikalischen Theorie inhaltsleer ist. Im Zuge dieser Argumentation wird der Ansatz der Via Negativa diskutiert, der eine alternative Lösung des Definitionsproblems des Physikalischen vorschlägt. Anhänger der Via Negativa versuchen nicht, "physikalisch" zu definieren, sondern gehen den Weg, das "Nicht-Mentale" zu definieren. Dabei muss keine Referenz mehr zu physikalischen Theorien gegeben werden. Dieser Ansatz wird schließlich wieder verworfen auf Basis einer Version von Hempels Dilemma, die auch auf die Via Negativa Anwendung findet.

Ein weiterer integraler Bestandteil der Definition des Physikalismus ist der Ausdruck "nichts anderes als" bzw. der Ausdruck "alles". Hier gibt es im physikalischen Lager eine Vielzahl an Möglichkeiten, wie die physikalische Natur der Dinge gesichert werden kann. Es wird in Kapitel 3 auf diese verschiedenen Möglichkeiten eingegangen und argumentiert, dass alle Spielarten des Physikalismus die Supervenienz des vermeintlich Mentalen auf dem Physikalischen als gemeinsamen Nenner haben. Davidson (1970) hat den Begriff der Supervenienz in die Diskussionen der Philosophie des Geistes eingebracht und darunter versteht man in einer allgemeinen Formulierung, dass es keine Veränderungen in vermeintlichen mentalen Facetten gibt ohne eine Änderung in physikalischen Facetten. Anders formuliert: Wenn zwei Personen komplett identisch in all ihren physikalischen Facetten sind, dann sind sie auch komplett identisch in all ihren mentalen Facetten. Diese Formulierung kann auch noch weiter generalisiert werden, sodass eine identische physikalische Aufmachung zwischen zwei Welten auch eine identische Aufmachung in allen anderen Facetten nach sich zieht, inklusive der mentalen Dinge, Eigenschaften, Relation und dergleichen. Ein bisher ungelöster Einwand gegen die Supervenienz ist das Blocker-Problem von Hawthorne (2002), das besagt, dass die Relation zwischen dem Physikalischen und dem Mentalen eigentlich schwächer ist als Supervenienz und die Supervenienzrelation durch sogenannte Blocker blockiert werden können. Nach Hawthorne kann der Physikalist die Möglichkeit von Blockern nicht ausschließen, weshalb Supervenienzformulierungen des Physikalismus, und auch logische stärkere Formulierung des Physikalismus wie die Identitätstheorie, problematisch macht. Ich werde Hawthornes Problem aufgreifen und zeigen, dass der Physikalist davon nicht betroffen ist, denn das Blocker-Szenario führt zu absurden Konsequenzen.

Da nach Kapitel 3 die Supervenienz als minimaler Physikalismus gerettet ist, gilt es noch festzumachen, ob Supervenienz allein reicht oder eine stärke Relation zwischen dem Mentalen und dem Physikalischen postuliert werden muss. In Kapitel 4 gehe ich den Weg, die stärkste Form der Relation zu verteidigen, nämlich die Identitätsrelation. Ich werde anhand zweier Kriterien wissenschaftlicher Theorien, die als oberste Entscheidungshilfe heranzuziehen sind, für 
die Reduktion des Mentalen auf das Physikalische im Sinne einer Identität zwischen dem Mentalen und dem Physikalischen auf Typenebene argumentieren. Zudem werde ich zeigen, dass eine Version des Physikalismus am besten als A-priori-Physikalismus verstanden werden soll, bei dem alle Fakten, Wahrheiten und Begebenheiten aus der Masse des physikalischen Wissens vom Schreibtisch aus abgeleitet werden können. Zu guter Letzt wird in diesem Kapitel gezeigt, dass ein konsequentes Weiterverfolgen der Argumentation, die zu einer TypenIdentitätstheorie a priori führt, auch zu einem semantischen Physikalismus führt. Ein semantischer Physikalismus wird hier in der Stoßrichtung des logischen Empirismus bzw. Positivismus verstanden, bei dem alles Mentale bereits in der Sprache der Physik ausgedrückt werden kann (Carnap, 1931, 1932; Hempfel, 1935). Als Haupteinwand gegen eine Identitätstheorie auf Typenebene wird gerne die multiple Realisierbarkeit vorgebracht. Als klassische Quelle dient hier Putnam (1967), der das wesentliche Problem darin sieht, dass Qualia und im Grunde alle mentalen Vorgänge in verschiedenen Spezies auch verschiedene neuronale Grundlagen aufweisen. Das gilt für Menschen im Vergleich zu Fledermäusen, aber sowohl interindividuell für verschiedene Personen hinweg als auch intraindividuell für dieselbe Person über verschiedene einzelne Zeitpunkte hinweg. Ich begegne Putnams Argument letztendlich damit, dass ein Einbeziehen gegenwärtiger empirischer Befunde aus den Naturwissenschaften und eine damit verbundene feinere Betrachtung der Realisierungsebene das Argument der multiplen Realisierbarkeit entkräftet.

In Kapitel 5 zeige ich, dass die Wahrheit meiner Identitätstheorie von dem Ausgang der Alltagspsychologie abhängt. Unter der Alltagspsychologie versteht man das alltägliche Erklären und Vorhersagen unseres Handelns, denn der Gang zum Kühlschrank wird durch mein Hungerfühl und meinem Wunsch, etwas zu essen, ausgelöst. Die Identitätstheorie setzt voraus, dass es eine Entsprechung zwischen der Alltagspsychologie und der Physik geben wird, sodass eine Reduktion geschehen kann. Im Gegensatz dazu behauptet der Eliminativismus, dass unsere alltagspsychologische Referenz auf Gefühle, Wünsche und dergleichen nicht funktioniert, weil alles Mentale eine bloße Illusion sei und es keine Entsprechung zwischen der Alltagspsychologie und Physik geben wird. Beide Positionen, die Identitätstheorie und der Eliminativismus, legen sich auf ein ganz bestimmtes Schicksal der Alltagspsychologie fest. Ich argumentiere, dass der Ausgang der Alltagspsychologie nicht festgemacht werden kann, und ich erarbeite einen Ansatz, in dem sich eine Reduktion und eine Elimination des Mentalen nicht gegenseitig ausschließen. Dabei entwickle ich einen liberalen A-priori-Eliminativismus, der genauso ontologisch sparsam wie die Identitätstheorie ist, sich darüber hinaus jedoch als begrifflich sparsamer und einfacher herausstellt. In Bezug auf die Explikation der These des Physikalismus schließe ich das fünfte Kapitel damit, meine Versionen der Identitätstheorie und des Eliminativismus als sich gegenseitig ergänzend anzusehen: Es ist entweder die Identitätstheorie oder der Eliminativismus wahr. 
Der Lieblingsgegner des Physikalisten ist der Dualist. Um die Diskussionspunkte zwischen beiden Lagern besser zu verstehen, gibt Kapitel 6 eine Übersicht der gängigsten Argumente. Damit werden einerseits die dualistischen Argumente (v.a. Jackson, 1982; Levine, 1983; Nagel, 1974; Chalmers, 1996; Nida-Rümelin, 2007) und andererseits die physikalistischen Argumente (v.a. Feigl, 1958/1967; Smart, 1959; Lewis, 1966; Armstrong, 1968; Papineau, 2002) dargelegt. Es wird ebenso darauf eingegangen, was eine dualistische Position in der Philosophie des Geistes tatsächlich ausmacht. In diesem Zusammenhang werden die Unterschiede zwischen einem Eigenschaft- und Substanzdualismus sowie zwischen einem interaktionistischen und epiphänomenalen Dualismus diskutiert. Bei einem interaktionistischen Dualismus wird mentalen Entitäten eine kausale Rolle zugeschrieben, sodass etwas Mentales auch etwas Physikalisches beeinflussen kann und damit eine Köper-Geist-Interaktion möglich wird. Hingegen behauptet der epiphänomenale Dualismus, dass mentale Entitäten Entitäten nicht kausal wirksam werden können.

Ob nun der Physikalismus, wie ich inn in dieser Arbeit entwickle, auch wahr ist, verfolge ich in Kapitel 7. Hierfür entwickle ich ein neues Argument, das ich das generalistische Argument für den Physikalismus nenne und das die (vorläufige) Wahrheit des Physikalismus als Konklusion eines deduktiven Arguments zeigt. Der Dreh- und Angelpunkt dieses Arguments ist der, dass der Physikalismus die überzeugendste Position in der Philosophie des Geistes ist, und er deshalb als wahr angenommen werden sollte. Nachdem ich für die Plausibilität der Prämissen argumentiert habe, antizipiere ich einen möglichen Einwand gegen mein Argument, der von Vertretern alternativer monistischer Positionen vorgebracht werden könnte, nämlich von Idealisten, neutralen Monisten und Panprotopsychisten. Sie könnten behaupten, dass sich mein generalistisches Argument für den Physikalismus, falls erfolgreich, lediglich gegen den Dualismus richtet, aber nicht gegen die monistischen Rivalen. Ich kontere diesem Einwand als Erstes durch verschiedene Probleme, welche die alternativen monistischen Positionen treffen, aber nicht den Physikalismus. Als Zweites zeige ich anhand wahrscheinlichkeitstheoretischer Überlegungen durch den Satz von Bayes (Bayes, 1764), dass dem Physikalismus im Vergleich zu den monistischen Rivalen eine höhere Wahrscheinlichkeit zukommt, wahr zu sein.

Die Arbeit schließt mit einer Konklusion in Kapitel 8, welche die Hauptpunkte der vorhergehenden Kapitel zusammenfasst und die wesentlichen Punkte nochmals darlegt. Die vorliegende Arbeit besteht auch aus drei Exkursen, die nicht unmittelbar für die Kernargumentation benötigt werden. Sie betrachten verschiedene Teile, Aussagen und Thesen in der Tiefe, führen sie weiter aus oder beleuchten sie in anderer, origineller Art und Weise. Die Exkurse greifen unter anderem auch Ideen der einzelnen Kapitel auf und entwickeln diese weiter, in einem teilweise sehr technischen Stil. Der Leser kann getrost die Exkurse bei einem ersten Lesedurchgang überspringen und bei Interesse später darauf zurückkommen. Die Hauptargumentation ist unabhängig von den angebotenen Exkursen, doch diese kann mitunter nach Durcharbeiten der entsprechenden Exkurse in einem anderen Lichte betrachtet werden. 


\section{Erster Exkurs E1: Die Intentionalität der Gemütsbewegungen bei Brentano}

Brentanos (1874/2008) ${ }^{3}$ bemerkenswertester Beitrag zur Philosophie des Geistes ist ohne Zweifel seine These der Intentionalität als Kennzeichen des Mentalen. Unter Intentionalität versteht man die Gerichtetheit von mentalen Zuständen. Es ist damit dasjenige gemeint, worauf sich der jeweilige mentale Zustand bezieht. So hat mein Wunsch, eine neue Gitarre zu kaufen, das Kaufen als seinen Inhalt, aber auch die Gitarre als seinen Inhalt. Meine Überzeugung, dass James T. Kirk der einzig wahre Kapitän der Enterprise ist, hat eben James T. Kirk als ihren primären Inhalt, aber auch weitere Inhalte wie beispielsweise die Enterprise. Die Idee, dass jeder mentale Zustand auf seinen Inhalt gerichtet ist, ist Brentanos zentrale These (S. 106):

Jedes psychische Phänomen ist durch das charakterisirt, was die Scholastiker des Mittelalters als die intentionale (auch wohl mentale) Inexistenz eines Gegenstandes genannt haben, und das wir, obwohl mit nicht ganz unzweideutigen Ausdrücken, die Beziehung auf einen Inhalt, die Richtung auf ein Object (worunter hier nicht eine Realität zu verstehen ist), oder die immanente Gegenständlichkeit nennen würden. Jedes enthält etwas als Object in sich, obwohl nicht jedes in gleicher Weise. In der Vorstellung ist etwas vorgestellt, in dem Urtheile ist etwas anerkannt oder verworfen, in der Liebe geliebt, in dem Hasse gehasst, in dem Begehren begehrt u. s. w.

Es ist demnach die Intentionalität, die den Unterschied zwischen der Klasse des Mentalen und der Klasse des Physikalischen ausmacht. An dieser Stelle ist es wichtig, den Begriff "Inexistenz" zu erklären. Damit ist nicht das Gegenteil von "Existenz" gemeint, so wie es ein moderner Sprachgebrauch suggerieren könnte, sondern mit einer Inexistenz ist im Sinne von Brentano ein mentales Innewohnen gemeint. Nach Brentano sind psychische Phänomene "das Hören eines Tones, das Sehen eines farbigen Gegenstandes, das Empfinden von Warm oder Kalt" (S. 9697). Im Vergleich dazu sind physische Phänomene "eine Farbe, eine Figur, eine Landschaft, die ich sehe; ein Accord, den ich höre; Wärme, Kälte, Geruch, die ich empfinde" (S. 97). Beim Hören eines Tones, beim Empfinden von Wärme und dergleichen ist von - psychischen Phänomenen die Rede, "welche intentional einen Gegenstand in sich enthalten" (S. 107) und "[d]asjenige Merkmal, welches die psychischen Phänomene unter allen am Meisten kennzeichnet, ist wohl ohne Zweifel die intentionale Inexistenz" (S. 115).

Was Brentano im Auge hat, ist die Intentionalität als notwendige und hinreichende Bedingung für das Mentale auszumachen. Intentionalität ist deswegen notwendig, weil ohne eine Gerichtetheit auf etwas auch keine mentalen Zustände vorhanden sind. Zusätzlich ist

\footnotetext{
${ }^{3}$ Alle Seitenangaben beziehen sich auf Brentanos Psychologie vom empirischen Standpunkt in der Ausgabe von 2008.
} 
Intentionalität auch hinreichend für etwas Mentales, denn wenn Intentionalität gegeben ist, ist auch etwas Mentales gegeben. Brentanos These der Intentionalität als Kennzeichen des Mentalen ist in der gegenwärtigen Literatur umstritten und es wird gegen diese Sichtweise argumentiert, dass Intentionalität eine notwendige Bedingung für etwas Mentales darstellt. Als ein beliebter Kritikpunkt wird das Vorhandensein von emotionalen Zuständen gebracht, wie beispielsweise ein allgemeines Unwohlsein, eine depressive Verstimmung und eine ungerichtete Angst. Bei diesen emotional gefärbten Erlebnissen scheint keinerlei Gerichtetheit vorhanden zu sein und nur das erlebte Gefühl spricht für das Vorhandensein eines psychischen Phänomens. Falls das der Fall ist, ist Intentionalität nicht notwendig für das Vorhandensein von mentalen Zuständen und Brentanos These kann in dieser strengen Form nicht aufrechterhalten werden. Als weitere Konsequenz wären emotionale mentale Zustände und intentionale mentale Zustände zwei verschiedene Klassen des Psychischen. Mein Hauptziel ist es, zu zeigen, dass Brentanos These nicht ohne Weiteres angenommen werden kann und die Frage zur Intentionalität von Gefühlen noch kein Ende gefunden hat. Doch bevor wir uns den vorgebrachten Gegenbeispielen zu Brentanos These widmen, werfen wir einen Blick darauf, warum er Wille und Gefühle (bzw. Emotionen) in eine Grundklasse des Psychischen zusammenfasst.

\section{E1.1. Gefühl und Wille als eine Grundklasse}

Das Zusammenfassen von Gefühl und Wille in eine Grundklasse verteidigt Brentano im achten Kapitel seiner Psychologie vom empirischen Standpunkt. Die Aufgabe dieser Klasse des Psychischen ist die Beurteilung des Wertes des intentionalen Gegenstandes. Entweder wird dieser als gut angenommen oder als schlecht abgelehnt. Brentano bringt folgende drei Argumente, warum Wille und Gefühl in eine Grundklasse fallen.

\section{Erstens kann keine scharfe Grenze zwischen dem Willen und dem Gefühl gezogen} werden. Brentano schreibt: "[U]nd ich weiss in Wahrheit nicht, wo die Grenze zwischen beiden Classen [Gefühl und Wille] eigentlich liegen sollte ... so zeigt sich auf dem gesammten Gebiete nirgends eine Kluft, sondern ganz allmälig finden die Uebergänge statt" (S. 256). Brentano zeigt anhand eines Beispiels, dass von der Traurigkeit (einem Gefühl) in kleinen Schritten bis zum Willensentschluss einer Tat (einem Willen) gegangen werden kann. So ist die Kette ausgehend von Traurigkeit, über Sehnsucht, Hoffnung, Verlangen und Mut bis schließlich zum Willensentschluss gekennzeichnet durch sanfte Übergänge.

Zweitens sind Unterschiede zwischen psychischen Phänomenen durch die Beziehung zum Objekt zu erklären. Innerhalb des Gefühls der Liebe gibt es Unterschiede in der Art und Weise, wie die Beziehung zum Objekt besteht. So bringt Brentano als Beispiel die Lust einer angenehmen Erwärmung und die Freude beim Anblick eines schönen Gemäldes. Diese 
psychischen Phänomene sind qualitativ verschieden, gehören aber dennoch zum Gefühl der Liebe, weil ungeachtet dieses qualitativen Unterschieds in beiden Fällen etwas als gut angenommen wird.

Drittens ist ein gleicher Unterschied der Endpole zwischen beliebigen gegensätzlichen Paaren zu finden. Der Gegensatz von Freude und Traurigkeit sowie Wollen und Nicht-Wollen ist im Grunde derselbe Gegensatz wie der von Lieb- und Unlieb-Sein. Der Gegensatz ist deswegen derselbe, weil die Distanz zwischen beiden Endpolen dieselbe ist: "[D]er Unterschied ist nicht grösser als der, welcher zwischen den Gegensätzen von Freude und Trauer, Hoffnung und Furcht, Muth und Verzagen, Verlangen und Fliehen und vielen anderen in Classe gefunden wird" (S. 272).

Doch was bei seiner Verteidigung der Zusammenfassung von Gefühl und Willen in eine Klasse unbeantwortet bleibt, ist die Frage der Intentionalität von Gefühlen. Damit die These der Intentionalität als das Kennzeichen des Mentalen als gesichert bleibt, muss noch gezeigt werden, dass ein Gefühl in gleicher Art und Weise Intentionalität aufweist wie ein Wille. In seinen drei Punkten zur Zusammengehörigkeit von Gefühl und Wille ist es schwer herauszulesen, wie die Intentionalität von Emotionen gesichert wird, ohne sie im Vorhinein einfach nur anzunehmen. Da die Intentionalität alles Mentalen Brentanos Kernthese ist, ist es auch unabdingbar, näher darauf einzugehen und seine These näher zu beleuchten. Dies soll im nächsten Abschnitt passieren.

\section{E1.2. Sind Wille und Gefühl doch zu unterschiedlich?}

Ein Teil einer Emotion ist ebenfalls eine gewisse Art und Weise, wie es sich anfühlt, in einem bestimmten emotionalen Zustand zu sein. So ist ein Angsterlebnis qualitativ verschieden zu einem Erlebnis des Verliebtseins. Dies ist in Nagels (1974) berühmter Formulierung das Wie-esIst eines bestimmten Erlebnisses, oder auch der phänomenale mentale Zustand, oder auch ein Quale. Die Frage ist nun, ob Qualia in gleicher Weise Intentionalität aufweisen wie beispielsweise der Wunsch nach etwas. Ein Angsterlebnis scheint, prima facie, nicht in gleicher Weise auf etwas gerichtet zu sein, wie es bei einem Wunsch der Fall ist. Beispielsweise sieht Searle (1983, S. 89) solch eine ungerichtete Angst als Gegenbeispiel zur These Brentanos, weil nicht jeder mentale Zustand Intentionalität aufweist:

Some, not all, mental states and events have Intentionality. Beliefs, fears, hopes, and desires are Intentional; but there are forms of nervousness, elation and undirected anxiety that are not Intentional ... My beliefs and desires must always be about something. But my nervousness and undirected anxiety need not in that way be about anything. 
Falls es mentale Zustände gibt, die nicht Intentionalität aufweisen, dann ist Intentionalität nicht notwendig für das Vorhandensein von mentalen Zuständen. Mentale Zustände können nach der Meinung einiger, wie beispielsweise Searle (1983), auch nicht-intentional sein, aber dafür phänomenal. Somit hätten wir zwei Arten mentaler Zustände: diejenigen, die Intentionalität aufweisen, und diejenigen, die Qualia haben. Das Vorhandensein von Intentionalität und Qualia wäre demnach für sich einzeln genommen eine jeweils hinreichende, aber keine für ein mentales Leben notwendige Bedingung. Doch, argumentiert Crane (1998), kann nur aufgrund der Tatsache, dass man das Worüber der scheinbar ungerichteten Angst nicht angeben kann, nicht die Nicht-Intentionalität der scheinbar ungerichteten Angst folgern. Zudem kann angegeben werden, ob diese Angst einen selbst oder andere betrifft. In diesem Sinne ist eine Gerichtetheit gegeben, denn im ersteren Fall ist die Angst auf einen selbst gerichtet und im letzteren Fall auf jemand anderen bzw. etwas anderes.

Eingeräumt, es scheint auch bei phänomenalen Zuständen eine gewisse Gerichtetheit gegeben zu sein. Doch ist diese Gerichtetheit dieselbe wie bei prototypischen intentionalen Zuständen? Bleiben wir bei der scheinbar ungerichteten Angst von vorher und nehmen wir an, dass der Inhalt dieser Angst die Welt als Gesamtes ist. Vielleicht ist man über den Weltfrieden besorgt und hat in diesem Sinne das ganze Weltengeschehen als eigentlichen Inhalt der ungerichteten Angst. Somit hat meine Angst die Erde als deren Inhalt. Vergleichen wir diesen Fall mit dem Wunsch, unsere Erde aus dem Weltraum zu beobachten. Auch hier ist die Erde der Inhalt, jetzt nämlich eines Wunsches. Scheint hier nicht ein wesentlicher Unterschied zu sein, wie man auf die Erde in diesen beiden Beispielen gerichtet ist? Bei dem Wunsch, die Erde aus dem Weltraum beobachten zu können, können wir uns ein Bild der Erde in der Art und Weise machen, wie es bei einer 1:1-Abbildung der Fall ist. Bei meiner Angst um die Erde scheint es nicht so zu sein, dass eine bloße 1:1-Abbildung der Erde den Inhalt meiner Angst treffend erfassen würde. Es fühlt sich vordergründig in gewisser Art und Weise an, sich Angst um die Welt zu machen, und dieses Angst-Quale ist durch eine intentionale Charakterisierung nicht erfasst. In diesem Sinne scheint ein Unterschied zwischen phänomenalen und intentionalen mentalen Zuständen vorhanden zu sein und der Einwand kann vorgebracht werden, dass die Intentionalität nicht diesen phänomenalen Aspekt in ihrer Gänze erfasst. Wenn ein phänomenaler Rest übrigbleibt, dann ist es nicht genug, bloß die intentionalen Aspekte zu charakterisieren, um unserem mentalen Leben gerecht zu werden.

Doch es ist auch argumentiert worden, dass eine Charakterisierung der Intentionalität von Emotionen ausreicht, um zu einer Charakterisierung von Qualia zu kommen. So ist beispielsweise bei Crane (1998, S. 251) zu finden:

Whatever the fate of qualia, we must accept that all mental states are permeated with intentionality, and characterising their phenomenal character - 
giving a phenomenology - can be achieved by characterising their

intentionality.

Nach dieser Position sind somit phänomenale mentale Eigenschaften ohne Rest auf intentionale mentale Eigenschaften zurückführbar, entweder durch die Identität von beiden oder durch eine Art Konstituierung des Phänomenalen durch das Intentionale. Damit dieser Versuch erfolgreich ist, muss jedoch auch gezeigt werden, wie bestimmte Emotionen, unter anderem das Beispiel der Besorgtheit über den Weltfrieden, gänzlich auf bestimmte intentionale Eigenschaften zurückgeführt werden können. In der gegenwärtigen Philosophie des Geistes erfreut sich die Idee, Qualia als nichts anderes zu sehen als dasjenige, was sie repräsentieren, erneuter Beliebtheit. Diese Position wird "Repräsentationalismus" genannt und sagt eben genau aus, dass Qualia auf Intentionalität zurückgeführt werden können ${ }^{4}$. Dieser Schritt entspricht Brentanos zentraler These, dass Intentionalität notwendig und hinreichend für etwas Mentales ist, und Brentano kann in diesem Sinne als Wegbereiter des Repräsentationalismus gesehen werden ${ }^{5}$. Im weiteren Schritte wird in der gegenwärtigen Literatur der Repräsentationalismus oftmals mit dem Physikalismus verbunden, sodass eine Repräsentation als nichts anderes als ein bestimmtes Erregungsmuster im Gehirn angesehen wird ${ }^{6}$. Brentanos These der Intentionalität hat somit immer noch einen wesentlichen Platz in der gegenwärtigen Philosophie des Geistes. Wie es letztendlich um das Schicksal der Intentionalität als das Kennzeichen des Mentalen steht, kann ich in diesem Exkurs nicht lösen, sondern ich habe lediglich auf das Problem für Brentanos These hingewiesen, dass nicht alle mentalen Zustände in gleicher Weise Intentionalität aufweisen müssen.

\section{E1.3. Konklusion}

Eine wichtige Frage ist also, was grundlegender von beiden ist: das Phänomenale oder die Intentionalität. Brentanos These der Intentionalität als das Kennzeichen des Mentalen verlangt, dass es die Intentionalität ist. Es ist nach Brentano die Gerichtetheit unserer mentalen Zustände, die das Ausschlaggebende für etwas Mentales ist. Nichtsdestotrotz ist auf der Basis von Qualia argumentiert worden, dass Intentionalität nicht eine notwendige Bedingung für etwas Mentales darstellt und das Beispiel von einer diffusen Besorgtheit zieht eine komplette Intentionalisierung

\footnotetext{
${ }^{4}$ Siehe dazu auch Kapitel 3.1.5, in dem der Repräsentationalismus weiter beleuchtet wird.

5 Siehe zu diesem Punkt Mulligan (2004), der eine Brücke zur gegenwärtigen Debatte in der Philosophie des Geistes schlägt und Brentano als eine Art Repräsentationalist ansieht: "Thus, Brentano combines the view that there are very many distinct qualia, for example the distinctive hues of different emotions, with representationalism" (S. 92). ${ }^{6}$ Für die Möglichkeit eines nicht-reduktiven Repräsentationalismus siehe z.B. Chalmers (2004). Chalmers argumentiert, dass das Phänomenale nicht durch bloße Charakterisierung der Intentionalität des jeweiligen mentalen Zustandes verstanden werden kann. Er sieht sowohl Qualia als auch Intentionalität als fundamentale, nichtreduzierbare Bestandteile unserer Welt und unseres mentalen Lebens an. Siehe jedoch auch Langsam (2020), der weder einem reduktiven noch einem nicht-reduktiven Repräsentationalismus Chancen einräumt, Qualia zu erklären.
} 
von Emotionen in Zweifel. Brentanos Beitrag zur Philosophie des Geistes erfährt in der aktuellen Diskussion in der Form des Repräsentationalismus wieder Aufschwung und es ist in diesem neuen Gewand, in dem die Frage zur Intentionalität von Gefühlen erneut aufgeworfen wird. 


\title{
With your feet on the air and your head on the ground
}

\author{
Where is my mind? \\ Where is my mind? \\ Where is my mind?
}

Pixies (1988) 


\section{Das Physikalische im Physikalismus}

Ich möchte in diesem Kapitel, erstens, eine Übersicht über einen neuen und innovativen Ansatz geben, der die Probleme einer Definition von "physikalisch" umgeht und stattdessen den scheinbar gangbareren Weg beschreitet, den Begriff "physikalisch" durch "fundamental nichtmental" zu ersetzen und Letzteres zu definieren. Anhänger dieses Ansatzes der Via Negativa argumentieren, dass dadurch Hempels Dilemma vermieden werden kann und eine negative Charakterisierung des Physikalischen den besten Weg darstellt. Zweitens werde ich argumentieren, dass Anhänger der Via Negativa die Bürde zuteil wird, nun eine adäquate Definition des fundamental Mentalen zu entwickeln. Doch jeglicher Versuch, solch eine Definition des Begriffes "mental" zu liefern, führt zum selben Dilemma wie auch die Definition des Begriffes "physikalisch". Somit ist die Via Negativa mit denselben Problemen und Einwänden konfrontiert, die sie eigentlich von vornherein vermeiden wollte. Drittens werde ich darlegen, warum eine Definition des Physikalischen über eine zukünftige physikalische Theorie die beste Option darstellt, damit eine bedeutungsvolle Diskussion in der Philosophie des Geistes vonstatten gehen kann.

\subsection{Verschiedene Definitionen des Physikalischen}

Es scheint so zu sein, dass diejenigen, die kaum mit der Philosophie des Geistes beschäftigt sind, kein großes Problem in der Beantwortung der Frage sehen, was schlussendlich als physikalisch zu gelten habe. Denn auch unser Alltagsverständnis sagt uns, dass Steine, Bäume, Wasser und dergleichen physikalische Dinge sind. Falls man sich nun auch die Frage stellt, was genau diese Dinge zu etwas Physikalischen macht, dann wäre die wahrscheinlichste Antwort, dass Steine, Bäume und Wasser Masse haben, denn sie wiegen etwas. Ebenfalls sind diese Dinge ausgedehnt, somit kann ich mich an innen stoßen, sie berühren, auf ihnen klettern oder sie trinken. Jemand könnte weiters auch sagen, dass man diese Dinge sehen kann, falls man vor innen steht, oder dass physikalische Dinge fest sind und deshalb nicht Wände passieren können. Damit ist die Beantwortung der ursprünglichen Frage beendet, denn nichts anderes muss mehr hinzugefügt werden. Wir wissen intuitiv, was es bedeutet, wenn etwas "physikalisch" ist und was paradigmatische physikalische Dinge wie Steine, Bäume und Wasser nun tatsächlich zu etwas Physikalischen macht, indem sie spezielle Eigenschaften haben wie Masse zu haben, ausgedehnt zu sein und fest zu sein. Diese Sichtweise ist die objektbasierte Definition des Physikalischen (Stoljar, 2001) und ist durch die Referenz zu unseren alltäglichen Intuitionen motiviert. Als Konsequenz bleibt übrig, dass all diejenigen Dinge, die nicht paradigmatische physikalische Dinge sind, nicht-physikalisch sind, wie auch immer eine weitere Charakterisierung des Nicht-Physikalischen aussieht. So weit so gut, aber als wie gut stellt sich 
diese intuitionsbasierte Definition des Physikalischen bei näherer Betrachtung heraus? Ich werde zeigen, dass es zumindest zwei Probleme mit der objektbasierten Definition gibt.

Das erste Problem ist, dass die objektbasierte Definition zirkulär ist, da das Physikalische durch eine Referenz zu paradigmatischen physikalischen Dingen definiert wird (siehe auch Dowell, 2006). Diese Definition kann wie folgt rekonstruiert werden: Die Eigenschaft $F$ ist genau dann physikalisch, wenn F charakteristisch für paradigmatische physikalische Dinge ist. Dabei wird das Definiendum "... F ist genau dann physikalisch" mit dem Definiens "... wenn F charakteristisch für paradigmatische physikalische Dinge ist" verbunden. Dadurch ist die charakteristische physikalische Natur der Dinge bereits hinreichend im Vorhinein verstanden worden, bevor eine explizite Definition des Physikalischen vorgelegt wurde, und das macht die objektbasierte Definition zirkulär. Um die objektbasierte Definition vor dem Vorwurf der Zirkularität zu bewahren, muss ein unabhängiges Verständnis von paradigmatischen physikalischen Dingen vorliegen ohne jegliche Referenz zu "physikalisch". Eine andere Möglichkeit ist, die Zirkularität zu akzeptieren, jedoch zwischen einem vitiösen Zirkel einerseits und einem hermeneutischen Zirkel andererseits zu unterscheiden. Ein vitiöser Zirkel in seiner einfachsten Form führt ein bestimmtes Objekt ein und definiert das eingeführte Objekt durch das eingeführte Objekt selbst. Beispiele sind unter anderem "Die Farbe Rot ist definiert durch die Eigenschaft, rot zu sein", oder "Die Farbe Rot ist definiert durch Rotheit". Solche Aussagen sind philosophisch uninteressant und ich kann mir keine Einsichten vorstellen, die daraus folgen. Im Vergleich dazu adelt ein hermeneutischer Zirkel die Zirkularität und definiert ein eingeführtes Objekt durch die gesamte Geschichte des eingeführten Objektes selbst, wobei das eingeführte Objekt notwendigerweise Teil seiner gesamten Geschichte ist. Man geht mit einem gewissen Vorverständnis in eine Thematik rein, hinterfragt einige Punkte davon, trifft neue Annahmen und gelangt dadurch zu einem besseren Verständnis. Daraufhin nehme ich das neu erlangte Verständnis wieder als Ausgangspunkt zugrunde, beginne wiederum zu hinterfragen, treffe wieder neue Annahmen und gelange so neuerlich zu einem besseren Verständnis. Somit kann ein Teil der Geschichte nicht ohne die gesamte Geschichte an sich verstanden werden. Typische Beispiele hermeneutischer Zirkel findet man in der Literatur, wo eine Textpassage nur durch die historischen Gegebenheiten zum Zeitpunkt des Schreibens, des vorherrschenden Zeitgeistes, verstanden werden kann und nicht aufgrund der Bedeutung der involvierten Wörter alleine. Solche Aussagen sind wiederum philosophisch interessant und hermeneutische Zirkel liefern somit gewisse Einsichten.

Vielleicht kann die objektbasierte Definition in diesem Sinne verbessert und repariert werden. Wenn dies der Fall ist, hätte man ein besseres Verständnis erreicht, doch ich fürchte, dass dies nicht reichen würde, da das zweite Problem für die objektbasierte Definition noch verheerender ist. Dieses zweite Problem kontrastiert die intuitionsgesteuerte objektbasierte Definition mit den gegenwärtigen Erkenntnissen aus den Naturwissenschaften, vor allem aus der Physik, die nicht im selben Sinne intuitionsgesteuert sind, sondern formaler und technischer 
sind. Betrachten wir die vorigen paradigmatischen physikalischen Dinge: Steine, Bäume und Wasser sowie ihre charakterisierenden Eigenschaften, nämlich eine Masse zu haben, ausgedehnt zu sein und Wände nicht passieren zu können. Nun betrachten wir irgendein physikalisches Ding, das sogleich in den Sinn kommt, und irgendeine Eigenschaft, die für dieses physikalische Ding charakterisierend ist. Wenn nun die Intuitionen über das betrachtete physikalische Ding und dessen charakterisierende Eigenschaft mit irgendeiner physikalischen Theorie über dasselbe Ding und dieselbe Eigenschaft verglichen wird, sind massive Unterschiede augenscheinlich. In der modernen Physik, aber auch grundsätzlich in allen Theorien innerhalb der Naturwissenschaften, werden viele kontraintuitive Dinge, Objekte, Partikel, Eigenschaften, Kräfte und dergleichen postuliert. Im Besonderen scheinen die Postulate der modernen Physik intuitiv und ironischerweise Beispiele fundamental mentaler Entitäten zu sein, die im herkömmlichen Sinne Dinge wie Götter, Seelen, Geister oder die Macht in Star Wars umfassen. Ich möchte diese Behauptung wie folgt verteidigen.

Beispielsweise werden Photonen als Partikel verstanden, die keine Masse haben und trotzdem Teil der Ontologie der Physiker sind. Im Lichte einer intuitionsbasierten Klassifikation sind Photonen keine paradigmatischen physikalischen Dinge und als Konsequenz wären sie nicht-physikalisch und fielen in die Klasse der fundamentalen mentalen Dinge. Doch das ist eindeutig falsch. Des Weiteren haben Photonen, und weitere Elementarteilchen, zusätzliche eigentümliche Eigenschaften. So verhalten sie sich einerseits wie klassische Teilchen und andererseits wie klassische Wellen, abhängig vom jeweiligen Experiment. Neutrinos, ein anderes Beispiel kontraintuitiver physikalischer Dinge, haben zwar eine bestimmte Masse, aber können durch Wände und alle anderen festen Gegenständen, auch Menschen, passieren. Diese Elementarteilchen müssten gemäß einer intuitionsbasierten Klassifikation ebenfalls in die Klasse der fundamentalen mentalen Dinge eingeordnet werden. Doch auch hier ist das klar falsch, da Neutrinos Teil der Ontologie der Physik sind. Um noch ein weiteres Beispiel anzufügen, sei auf moderne physikalische Theorien hingewiesen, die uns sagen, dass Atome in noch weitere kleinere Partikel geteilt werden können und so in Widerspruch zur klassischen Physik stehen. Unter diesen weiteren kleineren Partikeln sind Quarks zu nennen, die wiederum weitere eigentümliche Eigenschaften aufweisen wie beispielsweise eine elektrische Ladung von 2/3 und einen Spin von -1/2. Von intuitiver Seite her ist hier nochmals zu betonen, dass Quarks eigentlich nicht-physikalische Dinge sind, die in dieselbe ontologische Kategorie einzuordnen wären wie Götter, Geister, Seelen oder die Macht bei Star Wars. Also kann zusammengefasst die objektbasierte Definition zumindest Photonen, Neutrinos und Quarks nicht deren rechtmäßigen Platz in der Ontologie der Physik und der physikalistischen Geschichte der Welt zuweisen. Diese Konklusion ist auch bereits von der breiten Masse akzeptiert (z.B. Montero, $1999,2009)$ und der Hauptgrund ist das Aufbauen der objektbasierten Definition auf einem intuitionsgeleiteten Verständnis des Physikalischen. Schließlich bleibt die Suche noch bestehen, einen geeigneteren Ansatz zu finden, um das Physikalische zu definieren. 
Im Folgenden werde ich einen zweiten Ansatz vorstellen, den Stoljar (2001) die theoriebasierte Definition genannt hat, die sich von einem intuitionsgeleiteten Verständnis weg und hin zu einem formaleren und technischeren Verständnis entwickelt. Die Grundidee besteht darin, zu sagen, dass genau dann etwas als physikalisch gilt, wenn es auch von einer physikalischen Theorie beschrieben wird. Somit sind nur diejenigen Dinge physikalisch, die auch Teil einer auserkorenen physikalischen Theorie sind. An dieser Stelle muss noch ein nächster Punkt eingebracht werden, um auch diejenigen Dinge miteinzubeziehen, die nicht explizit in der physikalischen Theorie genannt werden, aber dennoch in einer spezifischen Relation zu den explizit genannten Dingen stehen. Diese spezifische Relation sichert und erklärt das Auftreten dieser physikalischen Dinge höherer Ordnung, sobald auch die physikalischen Dinge niedrigerer Ordnung fixiert sind. Die Literatur ist voll mit verschiedenen Ansätzen, wie diese spezifische Relation genauer beschrieben werden kann. Das ist an und für sich eine interessante Frage, doch hat sie in diesem Kapitel keine Bedeutung. Also kann für die vorliegenden Zwecke getrost die Lieblingsrelation ausgewählt werden, sei es nun Supervenienz, Realisierung, Identität oder etwas anderes. Somit sind Steine, Bäume oder Wasser auch nur dann physikalische Dinge, wenn sie von einer auserkorenen physikalischen Theorie explizit beschrieben und benannt werden oder sie in einer spezifischen Relation zu den explizit beschriebenen und benannten Postulaten der Theorie stehen. Aber was noch einer Entscheidung bedarf, ist die Frage, ob für eine theoriebasierte Definition eine gegenwärtige physikalische Theorie herangezogen werden soll, die man in Lehrbüchern, Fachjournalen oder anderen wissenschaftlichen Kommunikationsmedien findet oder eine zukünftige physikalische Theorie, die momentan noch nicht existiert und somit auch noch nicht in wissenschaftlichen Kommunikationsmedien zu finden ist.

Bevor die Frage der richtigen physikalischen Theorie analysiert wird, möchte ich noch auf einen möglichen grundlegenden Einwand antworten. Das Vorhaben, den Begriff "physikalisch" durch irgendeine physikalische Theorie zu definieren, erweckt prima facie den Verdacht eines Zirkelschlusses, weil ein Verständnis des Physikalischen bereits ein Verständnis der entsprechenden physikalischen Theorie voraussetzt. Darauf möchte ich entgegnen, dass eine theoriebasierte Definition auch als die Behauptung verstanden werden kann, dass das Physikalische durch eine Theorie definiert wird, die sich auf die beobachtbare Welt bezieht. In diesem Fall setzt ein Verständnis des Physikalischen nicht ein Verständnis einer physikalischen Theorie voraus, sondern ein Verständnis einer Theorie über die beobachtbare Welt. Eine Welt, die durch objektive Ursache-Wirkungs-Beziehungen geformt wird, ist genau das, worum es in einer Theorie über die beobachtbare Welt geht. Falls jemand nun trotzdem immer noch den Verdacht einer Zirkularität hegt, dann möchte ich in den sauren Apfel beißen. Wie ich in der Diskussion zur objektbasierten Definition erläutert habe, ist jedoch nicht jeder Zirkel vitiös. Somit ist eine Definition des Physikalischen durch eine physikalische Theorie, oder durch eine Theorie über die objektive Welt mit objektiven Ursachen und Wirkungen, trotzdem philosophisch 
interessant und liefert wertvolle Einsichten aufgrund des Vorhandenseins eines hermeneutischen Zirkels.

\subsection{Hempels Dilemma}

Das Vorhaben, zuerst die wesentlichen Begriffe zu definieren und erst darauf aufbauend in eine Diskussion innerhalb der Philosophie des Geistes zu treten, ist ein möglicher und legitimer Weg, um sich der Diskussion zu näheren. Melnyk (1997) war der Erste, der Hempels Problem den Namen "Hempels Dilemma" gegeben hat. Die Hauptpassage in Hempels Arbeiten zu seinem Dilemma spricht die Probleme an, die mit der Verwendung einer spezifischen physikalischen Sprache einhergehen, so auch mit der spezifischen physikalischen Sprache einer bestimmten physikalischen Theorie (Hempel, 1980, S. 194-195):

[T] he language of what physics is meant? Surely not that of, say, eighteenthcentury physics; for it contains terms like "caloric fluids", whose use is governed by theoretical assumptions now thought false. Nor can the language of contemporary physics claim the role of unitary language, since it will no doubt undergo further changes too. The thesis of physicalism would seem to require a language in which a true theory of all physical phenomena can be formulated. But it is quite unclear what is to be understood here by a physical phenomenon.

Hellman griff dieses Problem weiter auf und fasste Hempels Dilemma wie folgt zusammen (Hellman, 1985, S. 608):

[E]ither physicalist principles are based on current physics, in which case there is every reason to think they are false; or else they are not, in which case it is, at best, difficult to interpret them, since they are based on a "physics" that does not exit.

Neben diesen beiden klassischen Quellen zu Hempels Dilemma, formulierte Chomsky eine Facette des Dilemmas in seiner Diskussion des adäquaten Sprachgebrauches in der Philosophie des Geistes weiter aus. Interessanterweise diskutiert Chomsky diesen Punkt nicht im direkten Zusammenhang mit Hempels Schriften, sondern behandelt diesen Punkt eher als Nebenschauplatz in seinem Text (Chomsky, 2006, S. 86):

We can, however, be fairly sure that there will be a physical explanation for the phenomena in question, if they can be explained at all, for an uninteresting 
terminological reason, namely that the concept of "physical explanation" will no doubt be extended to incorporate whatever is discovered in this domain, exactly as it was extended to accommodate gravitational and electromagnetic force, massless particles, and numerous other entities and processes that would have offended the common sense of earlier generations.

Diese drei Quellen führen nun zu meiner Explikation von Hempels Dilemma. Vorweg möchte ich nochmals eine begriffliche Klarstellung geben. Zum Zwecke der vorliegenden Diskussion wird die Beziehung zwischen dem Physikalischen und Mentalen so angenommen, dass etwas Physikalisches als etwas Nicht-Mentales und etwas Mentales als etwas Nicht-Physikalisches verstanden wird. Entweder sind die postulierten Entitäten somit physikalisch oder mental, eine dritte Kategorie, wie beispielsweise die des Abstrakten, ist ausgeschlossen. Ein Grund für diese dichotome Betrachtungsweise ist auch der, dass in der Diskussion zwischen dem Physikalismus und dem Dualismus entscheidend ist, ob nun etwas physikalisch oder mental ist. Ob nun zusätzlich Abstrakta angenommen werden sollten, ist etwas, das in der Diskussion über die Wahrheit des Physikalismus keine Relevanz hat.

Das erste Horn von Hempels Dilemma besteht aus dem Bestreben, "physikalisch" durch Bezug auf die gegenwärtige Physik zu definieren. Als gegenwärtige Physik wird diejenige Physik verstanden, die tagesaktuell vorhanden ist und alle physikalischen Erkenntnisse zusammenfasst. Wenn sich nun "physikalisch" auf alle Entitäten bezieht, die durch die gegenwärtige Physik explizit postuliert werden oder die in einer spezifischen Relation zu den explizit postulierten Entitäten stehen, dann würde jegliche zukünftige Revision der Physik fundamental nicht-physikalische, also mentale Entitäten, postulieren.

Doch eine Induktion über die Wissenschaftsgeschichte zeigt, dass physikalische Theorien sich über die Zeit hinweg verändern, dass Wissenschaften fortschreiten und sich entwickeln, das wiederum zu neuen Entdeckungen führt. So beschreibt beispielsweise die klassische Newton'sche Physik Raum und Zeit als zwei separate Dinge. Zum einen verfließt Zeit kontinuierlich und ist unabhängig von jeglichen Geschehnissen innerhalb bestimmter Zeitfenster und zum anderen existiert Raum unabhängig von jeglichen Dingen innerhalb desselben. Das war die vorherrschende Theorie über Raum und Zeit bis Einsteins Konzept der Raumzeit den Weg in die Wissenschaften fand. In der modernen Physik sind Raum und Zeit eben nicht unabhängig voneinander, sondern bilden eine Einheit, sodass Ersteres nicht ohne Letzteres existiert und umgekehrt. Wir wissen heute, dass Einsteins Relativitätstheorie genauere Ergebnisse und Vorhersagen bringt als die klassische Physik. Die gegenwärtige Physik stützt sich sowohl auf das physikalische Standardmodell, das drei von vier fundamentale Kräfte abdeckt (d.h. die elektromagnetische Wechselwirkung, die schwache Wechselwirkung und die starke Wechselwirkung) als auch auf die Allgemeine Relativitätstheorie, welche die Gravitation als die vierte und letzte der fundamentalen Kräfte abdeckt. Da Wissenschaft ein sich 
entwickelndes Unterfangen ist, werden unter anderem neue Partikel, Kräfte, Eigenschaften und dergleichen entdeckt und neue Einsichten in die vier fundamentalen Kräfte lassen sich nur erwarten. Auf dessen Basis ist es wahrscheinlich, dass auch das ursprüngliche Standardmodell und die Allgemeine Relativitätstheorie nach und nach Revisionen unterliegen werden. Als Konsequenz ist nun deutlich, dass jegliche gegenwärtige Theorie innerhalb der Physik unvollendet und demnach streng genommen auch falsch ist.

Das zweite Horn von Hempels Dilemma besteht aus dem Bestreben, "physikalisch" durch Bezug auf eine ideale Physik zu definieren. Solch eine ideale Physik würde nicht mehr einer Revision bedürfen, würde dadurch vollendet sein und sofern der Physikalismus wahr ist, würde jede Wahrheit, jede Tatsache, jeder Sachverhalt in unserer Welt aus ihr folgen. Dies führt nun in meiner Analyse zu vier verschiedene Interpretationen des zweiten Horns. Erstens, der Physikalismus würde trivialerweise wahr werden, weil alles und jedes in der goldenen Zeit der Physik auch als physikalisch gelten würde, und nichts würde mehr in auch noch so ausgefeilten Experimenten gefunden werden, das nicht auch gleichzeitig in den physikalistischen Rahmen hineinfiele. Der Physikalismus würde aufgrund seiner Definition alles und jeden umfassen. Zweitens, der Physikalismus würde ohne jeglichen spezifischen Inhalt sein, weil wir jetzt noch nicht wissen, was nun Teil einer idealen Physik sein würde, und damit würden wir wieder bei unserem Ausgangsproblem ankommen, dass nämlich lediglich ein intuitives Verständnis des Physikalismus gegeben ist. Drittens, indem alles und jedes in die Physik aufgenommen wird, bleibt auch die Gefahr übrig, dass letztendlich ebenso etwas fundamental Mentales in die Physik aufgenommen wird. Ohne weitere Einschränkungen, was im Prinzip in eine physikalische Theorie aufgenommen werden soll, bleibt diese Interpretation bestehen. Viertens, wenn eine finale Physik alles in unserer Welt umfassen würde, würde auch nichts diese Theorie falsifizieren können, sie wäre immun gegen jeglichen Widerlegungsversuch. Die Moral von Hempels Dilemma ist nun folgende. Der Physikalismus ist falsch, wenn er durch einen Bezug auf eine gegenwärtige physikalische Theorie definiert wird, und (a) er ist trivialerweise wahr oder (b) er ist inhaltsleer oder (c) er beinhaltet fundamental Mentales oder (d) er ist nicht falsifizierbar, wenn er durch einen Bezug auf eine ideale physikalische Theorie definiert wird. Um die Folgerichtigkeit dieses Arguments besser analysieren zu können, ist folgende Explikation hilfreich (für eine andere Explikation ist z.B. auf Stoljar, 2010, S. 97-98 hingewiesen):

(1) "Physikalisch" wird entweder durch Bezug auf eine gegenwärtige physikalische Theorie oder durch Bezug auf eine ideale physikalische Theorie definiert.

(2) Wenn "physikalisch" durch Bezug auf eine gegenwärtige physikalische Theorie definiert wird, dann ist der Physikalismus falsch. 
(3) Wenn "physikalisch" durch Bezug auf eine ideale physikalische Theorie definiert wird, dann ist der Physikalismus

(3a) trivialerweise wahr oder

(3b) inhaltsleer oder

(3c) beinhaltet fundamental Mentales oder

(3d) ist nicht falsifizierbar.

(4) Entweder ist der Physikalismus falsch oder er ist trivialerweise wahr oder inhaltsleer oder er beinhaltet fundamental Mentales oder er ist nicht falsifizierbar.

Mit dieser vorliegenden Darstellung des Dilemmas ist es einfach, zu sehen, dass (4) logisch von (1) bis (3) folgt. Somit ist der einzige Weg, Hempels Dilemma zu besiegen, eine der Prämissen zu verwerfen. Ich werde nachfolgendend auf einen der Einwände gegen Hempels Dilemma eingehen, der als die Via Negativa bekannt ist und gegen Prämisse (1) gerichtet ist ${ }^{7}$.

\subsection{Gegen die erste Prämisse: die Via Negativa}

Als eine Reaktion auf die langwierige Debatte zwischen dem Physikalismus und konkurrierenden Theorien in der Philosophie des Geistes sowie auf die zur Debatte zugehörigen begrifflichen Probleme, lädt uns Barbara Montero in ihren Arbeiten ein, unsere Sichtweise auf das Körper-Geist-Problem zu verändern. Ihre wesentlichste Motivation zu diesem Schritt ist die, einen Neustart der Debatte zu gewährleisten, indem das klassische physikalistische Vokabular mit all seinen Problemen zurückgewiesen wird. Montero (1999, S. 194) beschreibt ihr Anliegen folgendermaßen:

So, perhaps, rather than worrying about whether the mind is physical, we should be concerned with whether the mind is ultimately non-mental. And this, I should mention, is a concern that has nothing to do with what current physics, future physics, or a final physics says about the world.

Das ist die Stelle, an der die Via Negativa eingeführt wird. Sie besagt, dass wir ein Verständnis des Physikalischen bekommen, indem wir definieren, was es nicht ist, nämlich es ist nicht etwas Mentales. Dadurch wird Prämisse (1) in Hempels Dilemma herausgefordert, weil ein Bezug auf

\footnotetext{
${ }^{7}$ Hinsichtlich der ersten Prämisse ist ein möglicher Einwand gegen (1) bereits zu Beginn dieses Kapitels genannt worden, als zwischen der objektbasierten Definition und der theoriebasierten Definition des Physikalischen unterschieden wurde. Im Prinzip würde eine objektbasierte Definition, falls sie erfolgreich formuliert werden würde, Prämisse (1) widerlegen und Hempels Dilemma zu Fall bringen. Doch wie ich zuvor argumentiert habe, scheitert jegliche intuitionsgeleitete Definition des Physikalischen durch die Referenz auf paradigmatische physikalische Objekte aufgrund eines Zirkelschlusses und vor allem aufgrund der falschen ontologischen Klassifikation von Photonen, Neutrinos und Quarks, die kontraintuitive physikalische Dinge sind.
} 
eine gegenwärtige oder eine ideale Physik nicht mehr die einzigen Wege sind, den Begriff "physikalisch" zu definieren. Monteros (1999, 2001) Hauptargument für die Via Negativa ist die Vermeidung von Hempels Dilemma durch das Vermeiden einer expliziten Definition von "physikalisch", indem "physikalisch" durch "fundamental nicht-mental" ersetzt wird. Wenn ich in der restlichen Arbeit von "fundamental" spreche, dann stimme ich mit Montero überein und verstehe etwas als fundamental, falls (i) es nicht auf irgendetwas anderes reduziert werden kann und falls es (ii) ein grundlegender Bestandteil von irgendetwas anderes ist ${ }^{8}$. Wenn ich fortan abgekürzt und vereinfacht "mental" verwende, meine ich letztendlich, was Montero gemeint hat, nämlich das fundamental Mentale, oder das genuine Mentale, oder das Mentale sui generis.

Spurrett und Papineau (1999) haben bereits die Via Negativa in der Diskussion weiterer klassischer Themenbereiche aufs Tableau gebracht. Sie waren die Ersten, welche die Via Negativa mit dem kausalen Argument für den Physikalismus (das sogenannte kausale Argument) in Verbindung gebracht haben. Die kontroverse Prämisse im kausalen Argument stellt die kausale Geschlossenheit des Physikalischen dar und besagt, dass jeder physikalische Effekt auch einen notwendigen und hinreichenden physikalischen Grund hat und somit kein Grund besteht, das physikalische Reich zu verlassen, um einen physikalischen Effekt zu erklären. An dieser Stelle möchte ich den Begriff der Überdeterminiertheit einführen. Wenn ein Ereignis überdeterminiert ist, dann verursachen zwei hinreichende Gründe dasselbe Ereignis. Ein Beispiel verwendend bedeutet das, dass ein Jäger mit seiner Schrotflinte auf ein Tier schießt, wobei ein Teil der Schrotkugeln den Kopf und der andere Teil der Schrotkugeln das Herz des Tieres trifft. Beide Verletzungen sind für sich genommen hinreichend für das Erlegen des Tieres. Es kann geschehen, dass ein Teil der Schrotkugeln in den Kopf und der andere Teil in das Herz trifft, doch das Problem dabei ist, dass diese Ausnahme bei Annahme einer Überdeterminiertheit zur Regel wird. Somit scheint es vernünftiger zu sein, eine systematische Überdeterminiertheit abzulehnen, was wiederum zur Folge hat, dass es in der gesamten kausalen Geschichte keinen Platz für mentale Ursachen gibt, die physikalische Effekte haben. Papineau (2002) kommt in seinem kausalen Argument zum Schluss, dass immer dann, falls eine vermeintlich mentale Ursache einen Effekt in unserer physikalischen Welt hat, dann ist diese vermeintliche mentale Ursache tatsächlich eine physikalische Ursache. A fortiori, durch die Kombination des kausalen Arguments mit dem Ausschluss einer systematischen Überdetermination darf geschlossen werden, dass alles, was physikalische Effekte hervorbringt, auch tatsächlich ontologisch physikalisch ist.

Spurrett und Papineau sind sich dem Problem einer expliziten Definition von "physikalisch" bewusst, da auch der Begriff "physikalisch" eine tragende Rolle in der kausalen

\footnotetext{
${ }^{8}$ Montero (2006) diskutiert ebenfalls die Implikationen für den Physikalismus, falls kein fundamentales Level erreicht wird und eine nicht-grundlegende physikalische Entität durch unendlich andere nicht-grundlegende physikalische Entitäten bedingt ist. Ihre Konklusion ist, dass der Physikalismus trotz alledem eine substantielle Theorie darstellt, die wahr oder falsch sein kann, unabhängig von einem tatsächlich existierenden fundamentalen Level. Ähnlich argumentiert auch Fiorese (2016), der eine unendliche Kette an sich bedingenden nicht-grundlegenden physikalischen Entitäten in seiner Version der Via Negativa zulässt.
} 
Geschlossenheit des Physikalischen hat, und die beiden Autoren formulierten die kausale Geschlossenheit des Physikalischen im Lichte der Via Negativa um und ersetzten "physikalisch" mit "nicht-mental". Damit gelingt es, die Folgerichtigkeit des kausalen Arguments zu gewährleisten, ohne eine vollständige Definition des Physikalischen geben zu müssen. Spurrett und Papineaus kausale Geschlossenheit des Nicht-Mentalen besagt, dass jede nicht-mentale Wirkung auch eine notwendige und hinreichende nicht-mentale Ursache hat. Falls eine vermeintlich mentale Ursache einen Effekt in unserer nicht-mentalen Welt hat, dann ist diese vermeintliche mentale Ursache tatsächlich eine nicht-mentale Ursache. A fortiori, durch die Kombination des kausalen Arguments für den Nicht-Mentalismus mit dem Ausschluss einer systematischen Überdetermination darf geschlossen werden, dass alles, was nicht-mentale Effekte hervorbringt, auch tatsächlich ontologisch nicht-mental ist. Die Autoren argumentieren, dass in den empirischen Wissenschaften bisher genau solch eine mentale Ursache noch nicht gefunden wurde, insbesondere in der Physiologie, und wir somit gerechtfertigterweise die Wahrheit der kausalen Geschlossenheit des Nicht-Mentalen annehmen können.

Spurrett und Papineau sind jedoch kritisiert worden. Gillett und Witmer (2001) wenden gegen den Schritt "physikalisch" durch "nicht-mental" zu ersetzen ein, dass es eigentlich nicht hilfreich für das ursprüngliche kausale Argument für den Physikalismus ist, weil Hempels Dilemma an einem späteren Punkt wieder emporsteigt. Sie bringen vor, dass jede Körperbewegung durch vorhergehende nicht-mentale Bedingungen und Gesetze verursacht wird und dadurch jede Körperbewegung durch vorhergehende physiologische Bedingungen und Gesetze verursacht wird. Ihr Argument ist wie folgt (Gillett \& Witmer, 2001, S. 305):

Certainly, no working scientist goes through the trouble of saying that suchand-such bodily movements have 'non-mental' causes. Instead, the conditions found to determine such events are characterized positively, in physiological terms.

Hempels Dilemma ist nun deshalb unausweichlich, weil beobachtbare Körperbewegungen wiederum in physiologischen Begriffen erklärt werden. Falls "physiologisch" durch eine gegenwärtige Physiologie erklärt wird, dann ist der Physiologismus falsch. Falls "physiologisch" durch eine zukünftige und ideale Physiologie erklärt wird, dann ist der Physiologismus (a) trivialerweise wahr oder (b) inhaltsleer oder (c) beinhaltet fundamental Mentales oder (d) ist nicht falsifizierbar ${ }^{9}$.

Anknüpfend an diese Diskussion verteidigen Montero und Papineau (2005) die Via Negativa auf Basis eines physiologischen Arguments, ähnlich zu Spurrett und Papineau. Damit

\footnotetext{
${ }^{9}$ Ich möchte hier anmerken, dass im Kontext dieser Arbeit "physikalisch" im weiten Sinne verwendet wird und auch biologische, chemische und alle anderen Naturwissenschaften umfasst, inklusive der Physiologie. Somit kann "physiologisch" durch "physikalisch" ausgetauscht werden, ohne die Bedeutung der Diskussion im Wesentlichen zu verändern.
} 
wollen sie die kausale Geschlossenheit des Nicht-Mentalen untermauern (Montero \& Papineau, 2005, S. 234-235):

To put it simply, the physiological argument for CNM [completeness, i.e. causal closure, of the non-mental] is this: given that one would expect (fundamental) mental forces, if they exist, to turn up under physiological investigation, the evidence for CNM is our failure, after extensive and prolonged physiological investigation, to come across any mental forces.

Nach Montero und Papineau sollte die kausale Geschlossenheit des Nicht-Mentalen so lange akzeptiert werden, bis erste kausal wirksame mentale Entitäten gefunden werden. Wird all unser profundes Wissen der Physiologie betrachtet, so hat bisher kein einziger Forscher, kein Forschungsteam oder keine Forschungseinrichtung jemals die Behauptung aufgestellt, fundamental mentale Entitäten nachgewiesen zu haben. Also können wir, aufgrund des Fehlens von mentalen Entitäten auf Basis unserer physiologischen Wissenschaften, rational die kausale Geschlossenheit des Nicht-Mentalen annehmen. Das stellt den ersten Teil des Arguments von Montero und Papineau dar. Was es noch zu zeigen gilt, ist der zweite Teil des Arguments, nämlich der Weg von der kausalen Geschlossenheit des Nicht-Mentalen zum Sieg über Hempels Dilemma.

Montero und Papineau schaffen den zweiten Teil des Arguments durch eine Induktion über die Wissenschaften, um kausal wirksame mentale Entitäten auszuschließen. Der Kern ihres Standpunktes ist dieser: Sie verteidigen die Via Negativa gegen Hempels Dilemma durch dasselbe induktive Argument, mit dem sie auch die kausale Geschlossenheit des NichtMentalen verteidigt haben, und Induktionen haben nichts mit expliziten Definitionen zu tun. Es existiert somit auch nicht die Notwendigkeit einer Definition von "nicht-mental" und aufgrund dessen muss auch kein Bezug zu gegenwärtigen oder zukünftigen nicht-mentalen Theorien hergestellt werden, um alle physiologischen Ursachen für Körperbewegungen zu identifizieren. Es reicht aus, zu sagen, dass es auf Basis der momentanen Wissenschaftsdisziplin der Physiologie keine guten Gründe gibt, etwas Mentales zu akzeptieren. Also sind Formulierungen im Lichte der Via Negativa bezüglich der kausalen Geschlossenheit des Physikalischen bzw. des Nicht-Mentalen sicher vor Hempels Dilemma. Ich denke jedoch nicht, dass die Verteidigung der Via Negativa gegen Hempels Dilemma, so wie es Montero und Papineau bringen, auch tatsächlich erfolgreich ist. Mein Haupteinwand ist, dass die Argumentation von Montero und Papineau ein klares, explizites und ein der Intuition übersteigendes Verständnis des Mentalen voraussetzt. Denn wie kann man etwas Mentales in der kausalen Geschichte der Physiologie ausschließen, wenn man nicht genau weiß, wonach man konkret sucht? Zu welchen Konsequenzen ein expliziter Definitionsversuch des Mentalen führt, möchte ich nachfolgend untersuchen. 


\subsection{Das erneute Auftauchen von Hempels Dilemma}

Was ich in den vorigen Erläuterungen zeigen wollte, ist die Wichtigkeit, was wir genau unter "mental" verstehen. Ich werde zeigen, dass jegliche Versuche, das Mentale zu definieren, dieselben Probleme aufwerfen, die wir bereits aus den Versuchen kennen, das Physikalische zu definieren. Somit taucht Hempels Dilemma in der Definition des Mentalen abermals auf ${ }^{10}$. Bevor ein paralleles Dilemma für das Mentale vorgebracht werden kann, braucht es noch einige weitere Überlegungen. Ich möchte die Doktrin des Mentalismus als eine These einführen, die behauptet, dass es nichts anderes als etwas Mentales gibt. Das bedeutet auch, dass vermeintlich physikalische Objekte wie Steine, Bäume und dergleichen bei der Gültigkeit eines Mentalismus eigentlich fundamental mental sind. Anhänger dieser These nenne ich Mentalisten. Ebenso verwende ich "mental" in einem breiten Verständnis und beziehe mich damit auf psychiatrische, psychologische, philosophische sowie alle weiteren Erkenntnisse, die mit Qualia oder Intentionalität beschäftigt sind. Weiters bezieht sich der Begriff "mentale Wissenschaften" eben auf all diejenigen Wissenschaften, welche mentale Erkenntnisse produzieren, und umfassen somit unter anderem Disziplinen wie Psychiatrie, Psychologie und Philosophie. Ich bin mir bewusst, dass gegenwärtige Vertreter solch eines starken Mentalismus in der Minderheit sind. In der gegenwärtigen Literatur findet nicht der Begriff "Mentalismus" Anwendung, sondern der Begriff "Idealismus", um sich auf die These zu beziehen, dass alles mental sei. Hierfür gibt es sehr wohl Vertreter, welche einen Berkeley'schen (Berkeley, 1734) Idealismus überwunden haben und moderne Versionen vertreten (z.B. Kastrup, 2007; Foster, 2008; Goff, 2017; siehe auch den Sammelband von Goldschmidt \& Pearce, 2017). Der Grund, warum ich dennoch den Begriff "Mentalismus" verwende und auf die Spielart des starken Mentalismus setze, ist ein dialektischer. Mein Ziel ist, einen exakten Gegenspieler zum Physikalismus zu schaffen und das Wiederauftauchen von Hempels Dilemma so klar wie möglich um des Arguments willen zu zeigen $^{11}$.

${ }^{10}$ Es sei angemerkt, dass Hempels Dilemma ebenfalls auftaucht, falls jemand "nicht-mental" direkt definieren will, ohne eine vorige Definition von "mental". In diesem Fall würde sich der Nicht-Mentalismus entweder auf Entitäten gegenwärtiger nicht-mentaler Theorien beziehen, was den Nicht-Mentalismus falsch machen würde. Oder der Nicht-Mentalismus würde sich auf Entitäten einer vollendeten nicht-mentalen Theorie beziehen, welche den Nicht-Mentalismus zu etwas machen, der (a) trivialerweise wahr wäre oder (b) inhaltsleer wäre oder (c) etwas fundamental Mentales beinhalten würde oder (d) nicht falsifizierbare wäre. Nichts würde jedoch gewonnen werden, wenn "nicht-mental" anstelle von "physikalisch" verwendet werden würde, da ein Ausdruck lediglich durch einen anderen Ausdruck ersetzt werden würde. Siehe Judisch (2008) für eine detailreichere Abhandlung.

${ }^{11}$ Neben solch einer starken Form des Mentalismus kann auch eine schwächere Form des Mentalismus vertreten werden. Somit kann ein schwacher Mentalismus als diejenige These verstanden werden, die besagt, dass es zusätzlich zu allen physikalischen Entitäten auch mentale Entitäten gibt. Im Kontrast dazu ist ein starker Mentalismus auf alles Existierende gerichtet, weil alles Existierende mental ist. Hingegen ist ein schwacher Mentalismus auf alles Existierende innerhalb des mentalen Bereiches gerichtet, weil es insgesamt zwei Existenzbereiche gibt, nämlich den mentalen Bereich und den physikalischen Bereich. Falls nun jemand einen schwachen Mentalismus dem starken Mentalismus in der Diskussion vorzieht, würde dies in der gesamten Argumentation nichts ändern, weil auch dann Hempels Dilemma nochmals auftaucht. Nichts Wesentliches hängt an der Einführung des Mentalismus als These, dass ausschließlich Mentales existiert, oder alternativ als These, dass es etwas Mentales gibt. Siehe Exkurs 2 in Anschluss an dieses Kapitel, der einen schwachen Mentalismus in Bezug auf Hempels Dilemma genauer diskutiert. 
In Bezug auf das Mentale ist ein intuitives Verständnis bereits vorhanden. Montero (2001) sagt explizit, dass als fundamental mental Qualia und Intentionalität gelten ${ }^{12}$. In derselben Art und Weise argumentiert Fiorese (2016) in seiner Verteidigung der Via Negativa, wenn er die Annahme zugrunde legt, dass das Mentale durch Qualia und Intentionalität bereits hinreichend definiert ist. Benennen wir dieses intuitive Verständnis die objektbasierte Definition des Mentalen. Hier kann man unter einem "Objekt" vieles verstehen und darunter auch Eigenschaften, Relation und dergleichen einordnen. Es ist lediglich eine verkürzte Form, sich auf alles gleichsam als "Objekt" im Sinne der objektbasierten Definition zu beziehen. Qualia und Intentionalität haben bestimmte Eigenschaften, die sie zu etwas Mentalem machen. Man würde intuitionsgeleitet sagen, dass beide keine Masse haben bzw. masselos sind, denn sie wiegen nichts. Auch können beide Wände passieren, denn sie sind nicht ausgedehnt und nehmen keinen Platz im Raum ein. Diesen Sachverhalt könnte man wohl als ektoplasmisch bezeichnen. Zu sagen, dass dem subjektiven Gefühl, in Liebe zu sein, oder der Gerichtetheit meines Gedankens auf meine Ehefrau die Eigenschaften wie Masse zu haben und ausgedehnt zu sein zukommt, ist kontraintuitiv. Somit muss nichts mehr über das Mentale gesagt werden, denn wir wissen bereits, was mit "mental" gemeint ist und was paradigmatische mentale Objekte wie Qualia und Intentionalität überhaupt zu etwas Mentalem macht. Es ist die Zuschreibung von speziellen Eigenschaften wie masselos zu sein und ektoplasmisch zu sein. Als Konsequenz bleibt übrig, dass all diejenigen Dinge, die nicht paradigmatische mentale Objekte sind, nichtmental sind, wie auch immer eine weitere Charakterisierung des Nicht-Mentalen aussieht. Doch bei dieser objektbasierten Definition des Mentalen ergeben sich zwei Probleme.

Das erste Problem ist, dass der Vorwurf der Zirkularität wieder auftaucht und somit dasselbe Problem hervorgekehrt wird, das wir in der Diskussion der objektbasierten Definition des Physikalischen gesehen haben. Die objektbasierte Definition des Mentalen kann wie folgt rekonstruiert werden: Die Eigenschaft $F$ ist genau dann mental, wenn $F$ charakteristisch für paradigmatische mentale Dinge ist. Dabei wird das Definiendum "... F ist genau dann mental" mit dem Definiens "... wenn F charakteristisch für paradigmatische mentale Dinge ist" verbunden. Dadurch ist die charakteristische mentale Natur der Dinge bereits hinreichend im Vorhinein verstanden worden, bevor eine explizite Definition des Mentalen vorgelegt wurde. Vielleicht kann dieses Zirkularitätsproblem durch die Unterscheidung zwischen einem vitiösen und einem hermeneutischen Zirkel aufgelöst werden. Falls es aufgelöst werden kann, wird es dennoch nicht die objektbasierte Definition retten, weil das zweite Problem für diesen Ansatz noch vernichtender ist. Das zweite Problem besteht darin, dass paradigmatische mentale Objekte bereits Eigenschaften haben, die ebenso auf physikalische Objekte wie Photonen oder

12 Wenn man das Mentale noch weiter charakterisieren will, bietet sich noch folgende Unterscheidung zwischen mentalen Subjekten, mentalen Objekten und mentalen Geschehnissen an. Beispielsweise kann die Aussage "Markus hat Schmerzen" so analysiert werden, dass Markus das erlebende mentale Subjekt ist, der empfundenen Schmerz bzw. das Schmerz-Quale das mentale Objekt ist und der Vorgang des Schmerzwahrnehmens das mentale Geschehnis. 
Neutrinos zutreffen. Photonen sind masselos und Neutrinos können feste Gegenstände wie Wände passieren. Somit ist es schlichtweg falsch, die Eigenschaften "masselos zu sein" und "ektoplasmisch zu sein" exklusiv mentalen Objekten zuzuschreiben. Diejenigen Objekte, welche diese beiden Eigenschaften aufweisen, können entweder mental oder physikalisch sein. Die objektbasierte Definition des Mentalen kann also keine richtige Zuteilung zu den ontologischen Kategorien aufgrund der intuitiven Eigenschaftszuschreibungen garantieren, ähnlich wie es auch bei der objektbasierten Definition des Physikalischen der Fall war.

Als Ergebnis der Analyse treffen somit zwei parallele Probleme für die objektbasierte Definition des Mentalen zu, wie wir sie in der Diskussion der objektbasierten Definition des Physikalischen kennen. Es sind die Probleme der Zirkularität und der uneindeutigen ontologischen Zuordnung. Dies zeigt eine Unzulänglichkeit der objektbasierten Definition des Mentalen, wenn das Ziel ein klares Verständnis davon ist, was "mental" bedeutet. Ein Rückzug auf theoriebasierte Definitionen scheint nun der einzige nächste Schritt zu sein, bei dem es wiederum zwei Optionen gibt. Zum einen kann eine gegenwärtige mentale Theorie herangezogen werden, die in Lehrbüchern, Fachjournalen oder anderen wissenschaftlichen Kommunikationsmedien zu finden ist. Zum anderen kann eine zukünftige, vollendete mentale Theorie herangezogen werden, die momentan noch nicht existiert und noch nicht in der Fachliteratur konkret beschrieben ist.

Crook und Gillett haben sich im Speziellen auf den Vergleich zwischen mentalen und physikalischen Theorien eingelassen und kehren als deren Unterscheidungsmerkmale Folgendes hervor (2001, S. 355, Fußnote 27):
A potentially important question is still whether a version of Hempel's dilemma arises with regard to the issue of specifying the 'mental'? In response we should mark the important differences between (folk or scientific) psychology and physics. For these disciplines differ both in the scope of their goals, the maturity of their theories and hence the degree to which they undergo revisions. Given these points, we therefore do not yet see any analogue of such a dilemma in the case of the mental for the reasons outlined. Nonetheless we acknowledge the potential seriousness of this issue.

Die Autoren sagen ganz klar, dass die Theorienentwicklung zwischen mentalen und physikalischen Theorien verschiedenartig ist. Physikalische Theorien verglichen mit mentalen Theorien haben verschiedene Ziele (scope of their goals) und verschiedene Entwicklungsgrade (maturity of their theories), weshalb der Begriff "mental" nicht denselben Unbeständigkeiten einer Theorienentwicklung ausgesetzt ist, wie es der Begriff "physikalisch" ist (Gillett \& Witmer, 2001). Ich werde gegen Crook und Gillett argumentieren und zeigen, dass zwischen 
physikalischen und mentalen Theorien eine hinreichende Ähnlichkeit besteht, die bei theoriebasierten Definitionsversuchen von "mental" zu einem parallelen Hempel'schen Dilemma führt.

Hier ist es wichtig, zu betonen, dass die Definition von "mental" durch Bezug auf eine mentale Theorie nicht vitiös zirkulär ist, genauso wenig wie die Definition von "physikalisch" durch Bezug auf eine physikalische Theorie. Der Grund dafür ist, dass ein Bezug auf mentale Theorien ganz einfach so verstanden werden kann, dass das Mentale als dasjenige definiert wird, das durch eine Theorie über die subjektive Welt postuliert wird. Eine Welt, die durch subjektive Ursache-Wirkungs-Beziehungen geformt wird, ist genau dasjenige, worum es in einer Theorie der subjektiven Welt geht. Oder, falls immer noch der Verdacht einer Zirkularität besteht, dann bleibt das Verständnis des Mentalen trotzdem philosophisch interessant, weil es Einsichten liefert, die sich auf einen hermeneutischen und damit nicht-vitiösen Zirkel gründen.

Ich bringe nun meine Gründe vor, warum mentale Theorien denselben Unbeständigkeiten einer Theorienentwicklung ausgesetzt sind, wie es auch physikalische Theorien sind. Als Erstes weise ich auf Crook und Gillett hin, die lediglich behaupten, dass mentale und physikalische Theorien nicht in ihren Zielen und Entwicklungsgraden vergleichbar sind. Doch wo genau die Unterschiede liegen, wurde von beiden nicht erörtert. Ebenso machen sie nicht explizit, was die Ziele physikalischer oder mentaler Theorien sind oder wie deren Entwicklungsgrad bestimmt werden kann. Trotz des Fehlens dieser Erläuterungen nehme ich das Ziel physikalischer Theorien derart an, dass sie die Welt, in der wir leben, erklären wollen. Ich möchte an dieser Stelle nicht zu restriktiv sein, was als physikalische Theorie zu gelten hat, und inkludiere alle Theorien innerhalb der Naturwissenschaften. Das heißt, dass Botaniker das Wachstum von Pflanzen erklären wollen, Zoologen das Paarungsverhalten von Menschenaffen erklären wollen, Neurophysiologen die Reizübertragung im Gehirn erklären wollen, Chemiker die Struktur von Neurotransmittern und Physiker die Beschleunigung eines Apfels, der angeblich auf den Kopf von Newton fiel. Es kann getrost jede beliebige Theorie innerhalb eines Bereiches der Naturwissenschaften ausgewählt werden und es wird klar, dass das spezifische Ziel einer Theorie innerhalb eines Bereiches unterschiedlich ist zu einem spezifischen Ziel einer Theorie innerhalb eines anderen Bereiches. Doch was die Ziele aller Bereich gemeinsam haben, ist eine Erklärung zu liefern für Beobachtungen, indem deren Ursachen und Wirkungen gemessen werden. Dasselbe gilt auch für mehrere Theorien innerhalb desselben Bereiches wie beispielsweise der Physik. Sei es die klassische Physik von Newton oder die Allgemeine Relativitätstheorie von Einstein, beide haben geringfügig verschiedene Ziele, doch heruntergebrochen auf deren gemeinsames Ziel ist es die Erklärung bestimmter Beobachtungen unserer Welt, indem Ursachen und Wirkungen gemessen werden.

Hinsichtlich der Ziele mentaler Theorien lässt sich sagen, dass es auch hier genug Theorien gibt, welche die subjektive Welt durch Referenz auf Ursachen und Wirkungen zu erklären versuchen. Betrachten wir Depression als ein Beispiel innerhalb des psychiatrischen 
Bereiches. Aus der Sicht eines Kognitivisten führen negative Gedanken über die Welt, über einen selbst und über die Zukunft zu negativen Gefühlen, die definitorisch für eine Depression sind. Oder betrachten wir den Größenwahn, bei dem Patienten überzeugt sind, jemand Spezielles zu sein, das wiederum zu Gefühlen der Überlegenheit bis Allmacht führt. Im psychologischen Bereich gibt es beispielsweise Ekmans (1999) Modell der Basisemotionen. In diesem Emotionsmodell gibt eine begrenzte Anzahl an grundlegenden Emotionen, die bei allen Menschen vorhanden sind (z.B. Freude), und eine Vielzahl an weiteren Emotionen (z.B. Eifersucht), die sich aus einer spezifischen Kombination der Basisemotionen ergeben. Oder betrachten wir Barretts (2017) Emotionsmodell der begrifflichen Akte, in dem ein emotionales Erleben durch die Aktualisierung vergangener emotionaler Erlebnisse auf Basis der involvierten emotionalen Begriffe verursacht wird. Im philosophischen Bereich gibt es beispielsweise den Panpsychismus, der behauptet, dass alles Existierende auch fundamental mental ist. Im Lichte des Panpsychismus ist jede Ursache und jede Wirkung essentiell und fundamental mental zu einem gewissen Grade, denn es gibt nichts in dieser Welt, das nicht auch in irgendeiner Weise mental ist. Wie diese These wiederum weiterentwickelt und konkret ausformuliert werden kann, ist eine offene Frage in der Philosophie (Seager, 2019).

Somit sind die Ziele physikalischer und mentaler Theorien dieselben, weil beide Arten von Theorien darauf abzielen, unsere Welt mit all ihren Ursachen und Wirkungen zu erklären. Natürlich beschäftigen sich physikalische Theorien mit den Ursachen und Wirkungen der objektiven und beobachterunabhängigen Welt, wohingegen sich mentale Theorien mit den Ursachen und Wirkungen der subjektiven und beobachterabhängigen Welt beschäftigen. Der Grund ist ex hypothesi, dass mentale Theorien über Intentionalität und Qualia handeln. Aber dieser Unterschied ist nicht das Wesentliche hier, sondern der Parallelismus zwischen beiden Arten von Theorien und deren gemeinsames Ziel, etwas über Ursachen und Wirkungen in unserer Welt zu erklären.

Kommen wir auf den Entwicklungsgrad physikalischer Theorien zu sprechen, den ich als etwas verstehe, das auf die Elaboriertheit, die Überzeugungskraft und das detailreiche Abdecken vieler Themenbereiche abzielt. Auf meiner Liste naturwissenschaftlicher Theorien, die definitiv diesen Anforderungen in höchstem Maße erfüllen, taucht Darwins Evolutionstheorie, Kopernikus' Theorie des heliozentrischen Weltbildes, Wegeners Theorie der Plattentektonik und Einsteins Allgemeine Relativitätstheorie auf. Für viele Dekaden bis hin zu Jahrhunderten, wurden diese Theorien unzählige Male durch Experimente und Beobachtungen von Wissenschaftlern gestützt. Diese Experimente und Beobachtungen haben geholfen, die Theorien zu verbessern, sodass sie immer elaborierter wurden. Auch gegenwärtig werden immer weiter neue Erkenntnisse generiert, die ermöglichen, diese Theorien überzeugender zu machen und darüber hinaus neue Anwendungsbereiche zu finden, im Sinne einer Verbreiterung der Themenbereiche und einer tiefergehenden, detailreicheren Abdeckung bestehender Themenbereiche. Ausgehend von diesem Sachverhalt ist es sicher, anzunehmen, dass die 
Agenda der Wissenschaftler wohl lange nicht darin bestehen wird, die Entwicklung der Menschheit durch natürliche Selektion ein für alle Mal zu widerlegen oder die Erde nochmals in den Mittelpunkt des Universums zu stellen oder den Himalaya als Ergebnis der Kollision zwischen der indischen Platte mit der eurasischen Platte zu leugnen oder die Raumzeit anzuzweifeln.

Hinsichtlich des Entwicklungsgrades mentaler Theorien lässt sich sagen, dass es im mentalen Bereich ebenso vergleichbare Theorien hinsichtlich Elaboriertheit, Überzeugungskraft und detailreichem Abdecken vieler Themenbereiche gibt. Nicht nur seit Dekaden oder Jahrhunderten, sondern seit vielen tausend Jahren liegen Beobachtungen der Menschheit zur Intentionalität und zu Qualia vor, die wiederum die entsprechenden Theorien verbessern, sodass sie immer elaborierter wurden. Des Weiteren werden auch heutzutage laufend neue Erkenntnisse generiert, die beispielsweise kognitive Ansätze zu psychischen Störungen im psychiatrischen Bereich, Emotionstheorien im psychologischen Bereich und Ansätze des Panpsychismus im philosophischen Bereich überzeugender machen. Im gleichen Atemzug werden neue Anwendungsbereiche für mentale Theorien geschaffen im Sinne einer Erweiterung der Themenbereiche und einer tiefergehenden, detailreicheren Abdeckung bestehender Themenbereiche. Im Lichte dieses Sachverhaltes ist es auch hier sicher, anzunehmen, dass die bahnbrechenden Schlagzeilen der mentalen Wissenschaften wohl lange nicht darin bestehen werden, dass die kausale Verbindung zwischen bestimmten Gedanken und Gefühlen ein für alle Mal widerlegt wird oder die Möglichkeit verschiedener grundlegender Bestandteile, aus denen Emotionen gebildet werden, ausgeschlossen werden oder der Panpsychismus letztgültig widerlegt wird. Somit sind die Entwicklungsgrade physikalischer und mentaler Theorien dieselben, weil beide Arten von Theorien elaboriert sind, überzeugend sind und viele Themenbereiche detailreich abdecken.

Falls dennoch jemand denkt, warum auch immer, dass mein Standpunkt nicht haltbar ist und ich die Konklusion zu früh erreicht habe, biete ich folgende zwei Alternativen an. Erstens, zum Zwecke des Arguments, wenn eine schwache Nicht-Parallelität zwischen mentalen und physikalischen Theorien gilt, sodass mentale Theorien einen geringeren Entwicklungsgrad als physikalische Theorien haben, dann ist dieser Unterschied nur für gegenwärtige mentale Theorien gültig. So kann es ruhig sein, dass gegenwärtige mentale Theorien weniger entwickelt sind, denn das wäre keine problematische Tatsache. Es zeigt bestenfalls lediglich die Ungleichheit zum jetzigen Zeitpunkt, aber es sagt nichts über eine zukünftige, vollendete mentale Theorie aus. Als Konsequenz der fehlenden Analogie zwischen gegenwärtigen mentalen und physikalischen Theorien würde eine schwache Nicht-Parallelität das ursprüngliche Hempel'sche Dilemma zu einem einfachen Hempel'schen Argument gegen eine vollendete mentale Theorie degradieren, weil ein Bezug auf irgendeine gegenwärtige mentale Theorie nicht mehr möglich wäre. Solche ein Argument würde durch den Bezug auf eine vollendete mentale Theorie in der Definition des Begriffes "mental" die dazugehörige Doktrin des "Mentalismus" 
trivialerweise wahr machen oder als inhaltsleer hinstellen oder zu etwas machen, dass fundamental Physikalisches beinhaltet oder nicht falsifizierbar machen.

Zweitens, wenn eine starke Nicht-Parallelität zwischen mentalen und physikalischen Theorien gilt, sodass mentale Theorien weder zum gegenwärtigen Zeitpunkt noch zu irgendeinem zukünftigen Zeitpunkt vergleichbar hinsichtlich deren Entwicklungsgrade sind, dann wird die gesamte Motivation der Via Negativa unklar. Der Grund ist der, dass die Via Negativa nur unter der Annahme eines klaren Verständnisses des Mentalen Sinn macht. Da eine objektbasierte Definition des Mentalen nicht gangbar ist, muss auf eine theoriebasierte Definition zurückgegriffen werden. Doch wenn sowohl in Bezug auf gegenwärtige als auch auf zukünftige mentale Theorien kein hinreichend großer Entwicklungsgrad dieser Theorien festgemacht werden kann, dann gibt es einerseits auch keine Hempel'schen Argumente gegen theoriebasierte Definitionsversuche und andererseits verliert die Via Negativa ihre Argumentationsstärke, weil theoriebasierte Definitionsversuche die letzte Möglichkeit waren, ein genaues Verständnis des Mentalen zu erlangen. Ohne ein klares Verständnis ist auch keine Via Negativa möglich. Vielleicht will jemand dagegen einwenden, dass eine gering entwickelte mentale Theorie ausreicht, um ein hinreichend klares Verständnis des Mentalen zu bekommen. Ich antworte darauf, indem ich an den ursprünglichen Grund erinnere, warum die Via Negativa überhaupt vorgelegt wurde. Es war die Motivation, einen alternativen Ansatz vorzulegen, wie man das Physikalische definieren kann, nämlich durch den Umweg, das Mentale zuerst zu definieren und dadurch Hempels Dilemma zu entgehen. Aber nun ergibt sich, dass jede theoriebasierte Definition des Physikalischen, ex hypothesi, gut entwickelte Theorien verwendet, jedoch jede theoriebasierte Definition des Mentalen schlecht entwickelte Theorien verwendet. Unterm Strich tauschen im Grunde Anhänger der Via Negativa ein gut entwickeltes und reifes Verständnis des Physikalischen mit einem schlecht entwickelten und unreifen Verständnis des Mentalen. Selbstverständlich kann auch so Hempels Dilemma vermieden werden, doch ist der Preis dafür ein schlecht entwickeltes und unreifes Verständnis des Mentalen. Ich denke, dass dieser Preis zu hoch ist.

Die obige Diskussion zusammengefasst ist nun erfolgreich ein hinreichender Parallelismus zwischen mentalen und physikalischen Theorien aufgezeigt worden, zumindest in Bezug auf Crook und Gilletts Fußnote 27 und den zwei Merkmalen der Ziele und der Entwicklungsgrade der Theorien. Also kann eine Formulierung von Hempels Dilemma gegeben werden, die sich gegen die Via Negativa richtet ${ }^{13}$.

\footnotetext{
${ }^{13}$ In gleicher Weise wie beim ursprünglichen Hempel'schen Dilemma ist auch hier bereits ein Einwand gegen (1') erwähnt worden als zwischen der objektbasierten und der theoriebasierten Definition des Mentalen unterschieden wurde. Prinzipiell gilt auch hier, dass eine erfolgreiche objektbasierte Definition Prämisse (1') erfolgreich widerlegen würde und Hempels Dilemma damit besiegt wäre. Wie ich jedoch zuvor argumentiert habe, scheitert eine intuitionsbasierte Definition des Mentalen durch Bezug auf paradigmatische mentale Objekte aufgrund des Zirkularitätsproblems und der Unzulänglichkeit, zwischen mentalen und physikalischen Eigenschaften zu differenzieren.
} 
(1') "Mental" wird entweder durch Bezug auf eine gegenwärtige mentale Theorie oder durch Bezug auf eine ideale mentale Theorie definiert.

(2') Wenn "mental" durch Bezug auf eine gegenwärtige mentale Theorie definiert wird, dann ist der Mentalismus falsch.

(3') Wenn "mental" durch Bezug auf eine ideale mentale Theorie definiert wird, dann ist der Mentalismus

(3a') trivialerweise wahr oder

(3b') inhaltsleer oder

$\left(3 c^{\prime}\right)$ beinhaltet fundamental Physikalisches oder

(3d') ist nicht falsifizierbar.

(4') Entweder ist der Mentalismus falsch oder er ist trivialerweise wahr oder inhaltsleer oder er beinhaltet fundamental Physikalisches oder er ist nicht falsifizierbar.

Das erste Horn von Hempels Dilemma besteht aus dem Bestreben, "mental" durch Bezug auf die gegenwärtigen mentalen Wissenschaften zu definieren. An dieser Stelle möchte ich nochmals meine ursprüngliche begriffliche Klarstellung wiederholen. Etwas ist genau dann mental, wenn es nicht-physikalisch ist, und etwas ist genau dann physikalisch, wenn es nichtmental ist. Eine dritte Kategorie, wie die der Abstrakta, ist ausgeschlossen. Das trifft auch insbesondere deshalb zu, weil sich das parallele Hempel'sche Dilemma gegen die Via Negativa richtet, die ebenso diese Dichotomie voraussetzt.

Wenn sich nun "mental" auf alle Entitäten bezieht, die durch die gegenwärtigen mentalen Wissenschaften explizit postuliert werden oder die in einer spezifischen Relation zu den explizit postulierten Entitäten stehen, dann würde jegliche zukünftige Revision der mentalen Wissenschaften fundamental nicht-mentale, also physikalische, Entitäten postulieren. Doch eine Induktion über die Wissenschaftsgeschichte zeigt, dass mentale Theorien sich über die Zeit hinweg verändern, fortschreiten und sich entwickeln, was wiederum zu neuen Entdeckungen führt. So waren beispielsweise die Gedanken unserer Vorfahren auf den Erfolg der nächsten Jagd gerichtet und wenn dieses Ziel erreicht wurde, breiteten sich positive emotionale Zustände wie Freude, Stolz und Zufriedenheit aus. Diese und andere intentionale und qualitative Zustände unseres mentalen Lebens waren stets anzutreffen, bis besser elaborierte Theorien in Abhängigkeit unserer gegenwärtigen Lebenswelt entwickelt wurden und eine weitere Betrachtung zuließen. Beispielsweise haben Menschen nun einen vielfältigeren und größeren Zugang zu Dingen, auf die unsere Gedanken gerichtet werden können. Darunter fallen unter anderem Autos oder Ideen von Philosophen, mit denen man gerade beschäftigt ist, die weiters noch detailreicher betrachtet werden können. Eine detailreichere Betrachtung eines Autos 
umfasst die Gerichtetheit auf den hochglänzenden, polierten, metallic-glänzenden oder matten Lack in der Farbe Bordeaux und die subjektive Erlebnisqualität, die mit dessen Betrachtung einhergeht. Zudem kann eine viel größere Bandbreite an Emotionen erlebt werden, wie beispielsweise die Freude eines Lottogewinnes oder die Trauer einer Scheidung. Der wesentliche Punkt dabei ist, dass intentionale Zustände (Gedanken) und qualitativ erlebbare Zustände (Emotionen) immer mehr wurden, sodass sie auch immer mehr Themenbereiche abdecken und diese Themenbereiche wiederum in größerer Detailtiefe abdecken. Innerhalb verschiedener mentaler Disziplinen wissen wir momentan mit größerer Genauigkeit, wie sich Gedanken gegenseitig beeinflussen (z.B. bei kognitiven Ansätzen der Psychiatrie), wie sich emotionale Erlebnisse gegenseitig beeinflussen (z.B. Emotionstheorien in der Psychologie) und wie eine Welt, in der es fundamentale Mentalität gibt, vorstellbar ist (z.B. panpsychistische Ansätze in der Philosophie). Als Konsequenz, "mental" durch irgendeine gegenwärtige mentale Theorie zu definieren, ist nun deutlich, dass jegliche gegenwärtige Theorie innerhalb der mentalen Wissenschaften unvollendet und demnach streng genommen auch falsch ist.

Das zweite Horn von Hempels Dilemma besteht aus dem Bestreben, "mental" durch Bezug auf eine ideale mentale Wissenschaft zu definieren. Solch eine ideale mentale Wissenschaft würde keiner Revision mehr bedürfen, würde dadurch vollendet sein und jede Wahrheit, jede Tatsache, jeder Sachverhalt in unserer Welt würde aus ihr folgen. Dies führt nun in meiner Analyse zu vier verschiedenen Interpretationen des zweiten Horns. Erstens, der Mentalismus würde trivialerweise wahr werden, weil alles und jedes in der goldenen Zeit der mentalen Wissenschaft auch als mental gelten würde, und nichts würde mehr in auch noch so ausgefeilten (Gedanken-)Experimenten gefunden werden, das nicht auch gleichzeitig in den mentalistischen Rahmen hineinfiele. Der Mentalismus würde aufgrund seiner Definition alles und jeden umfassen. Zweitens, der Mentalismus würde ohne jeglichen spezifischen Inhalt sein, weil wir jetzt noch nicht wissen, was nun Teil einer idealen mentalen Wissenschaft sein würde und damit würden wir wieder bei unserem Ausgangsproblem ankommen, dass nämlich lediglich ein intuitives Verständnis des Mentalismus gegeben ist. Drittens, indem alles und jedes in die mentale Wissenschaft aufgenommen wird, bleibt auch die Gefahr übrig, dass letztendlich ebenso etwas fundamental Physikalisches in die mentale Wissenschaft aufgenommen wird. Ohne weitere Einschränkungen, was im Prinzip in eine mentale Theorie aufgenommen werden soll, bleibt diese Interpretation bestehen. Viertens, wenn eine finale mentale Wissenschaft alles in unserer Welt umfassen würde, würde auch nichts diese Theorie falsifizieren können, sie wäre immun gegen jeglichen Widerlegungsversuch.

Die Moral von Hempels Dilemma ist nun folgende. Der Mentalismus ist falsch, wenn er durch einen Bezug auf eine gegenwärtige mentale Theorie definiert wird, und (a) er ist trivialerweise wahr oder (b) er ist inhaltsleer ist oder (c) beinhaltet fundamental Physikalisches oder (d) er ist nicht falsifizierbar, wenn er durch einen Bezug auf eine ideale mentale Theorie definiert wird. Also ist das Austauschen des Begriffes "physikalisch" durch "mental" um nichts 
besser in der Charakterisierung des Physikalischen bzw. Nicht-Mentalen, weil der Begriff "mental" in "nicht-mental" ebenfalls zu Hempels Dilemma führt, genauso wie es auch ursprünglich beim Begriff "physikalisch" war. Ohne Hempels Dilemma besiegen zu können, stellt die Via Negativa nicht die bessere Strategie dar, für die sie anfangs gehalten wurde. Es scheint, dass wir uns mit einer intuitiven Charakterisierung des Physikalischen begnügen müssen. Aber hier taucht die altbekannte Frage auf, wie wir schlussendlich entscheiden können, ob eine physikalistische oder dualistische Position in der Philosophie des Geistes wahr ist. Ich möchte dem Leser eine optimistische Lösung anbieten, warum Physikalisten getrost das Physikalische durch Bezug auf eine ideale Physik definieren und gleichzeitig Prämisse (3) von Hempels Dilemma entkräften können.

\subsection{Eine Definition von "physikalisch"}

Wenn Definitionsversuche zum Begriff "mental" nicht besser sind als Definitionsversuche von "physikalisch", dann stehen prinzipiell wieder Möglichkeiten einer theoriebasierten Definition des Physikalischen offen. Solch eine theoriebasierte Definition ist über gegenwärtige physikalische Theorien nicht gangbar, weil überzeugende Gründe in der Literatur vorhanden sind, welche die gegenwärtige Physik zu einer Sackgasse machen ${ }^{14}$. Die Prämisse (2) von Hempels Dilemma bleibt somit aufrecht. Der beste Weg ist, über eine ideale Physik zu gehen und Prämisse (3) von Hempels Dilemma zu entkräften.

Ich möchte als Erstes mit dem Vorwurf der Inhaltsleere in (3b) beginnen und zeigen, warum dieser Vorwurf zurückgewiesen werden muss. Eine Replik ist die rationale und induktionsbasierte Annahme, dass wir eine gewisse Ahnung davon haben, wie eine ideale Physik aussehen wird, weil die Form und Struktur dieselbe bleibt. Wenn ich über Form und Struktur spreche, dann meine ich damit das Vorhandensein bestimmter Kernannahmen einer Theorie, welche die Deduktion bestimmter Sachverhalte erlaubt. So waren in der klassischen Physik Raum und Zeit getrennte Größen bis die Allgemeine Relativitätstheorie daraus die einzelne Größe der Raumzeit machte. Beide Ansätze bildeten den Kern der jeweiligen Theorie, aus denen wiederum verschiedene Sachverhalte und überprüfbare Hypothesen folgen. Vielleicht nimmt eine ideale Physik eine andere Position zu Raum und Zeit ein, doch wie auch immer diese Position aussehen möge, es ändern nichts daran, dass auch diese ein Kern der idealen

\footnotetext{
${ }^{14}$ Siehe vor allem Melnyk (1997, 2003), der sich für das Hinwenden auf gegenwärtige physikalische Theorien ausspricht. Die theoriebasierte Definition durch Bezug auf gegenwärtige physikalische Theorien im Allgemeinen und Melnyks elaborierter Ansatz im Speziellen wurde umfassend in der Literatur diskutiert und deshalb werde ich die Diskussion nicht wiederholen. Kurz zusammengefasst: Melnyk argumentiert, dass eine gegenwärtige physikalische Theorie nicht wahr sein muss, sondern lediglich eine höhere Wahrscheinlichkeit als konkurrierende Theorien aufweisen muss, wahr zu sein. Das überzeugendste Argument gegen Melnyk ist der Standpunkt, dass die Physik von morgen bereits eine höhere Wahrscheinlichkeit aufweist, wahr zu sein, verglichen mit der Physik von heute. Damit ist auch Melnyks Konzept der Wahrheitsnähe nicht adäquat, um das Physikalische durch gegenwärtige Theorien zu definieren, und Prämisse (2) von Hempels Dilemma ist ungeschlagen. Für weitere Diskussion zu diesem Punkt verweise ich insbesondere auf Crook und Gillett (2001).
} 
Physik darstellt mit entsprechenden Folgerungen daraus. Des Weiteren ist auch jegliche physikalische Theorie inhaltlich mit der objektiven Welt beschäftigt und den darin vorhandenen Ursache-Wirkung-Beziehungen. Es werden die Fragen neuerlich und präziser beantwortet, wie beispielsweise die Frage nach den Wechselwirkungen zwischen bestimmten Elementarteilchen untereinander, die Frage nach der erfolgreichen Energiegewinnung bzw. Antriebsmöglichkeit für einen Weltraumflug mit Lichtgeschwindigkeit und die Frage nach der Fallgeschwindigkeit von Äpfeln im gebogenen Raum. Dies alles ist hinreichend, um die Brauchbarkeit einer finalen Theorie für die vorliegenden Zwecke positiv zu bewerten. Es muss jedoch eingeräumt werden, dass wir selbstverständlich noch nicht wissen, welche konkreten Postulate, Kräfte, Relationen und dergleichen vorhanden sein werden, doch das soll kein Problem darstellen. Wesentlich ist, dass wir zumindest eine Einsicht in bestimmte Aspekte einer idealen Physik haben und dadurch der Vorwurf, wir hätten überhaupt keine Idee, wie eine ideale Physik nur irgendwie aussehen könnte, abgewiesen werden kann.

Als Zweites möchte ich gemeinsam in einer Gruppe den Vorwurf der Trivialität in (3a), den der Mentalität in (3c) und den der Nicht-Falsifizierbarkeit in (3d) aufgreifen, da alle drei eng miteinander zusammenhängen. Hinsichtlich des Trivialitätsvorwurfes möchte ich an den Status des Physikalismus als eine metaphysische These erinnern, dessen Wahrheitswert jedoch davon abhängt, wie die aktuale Welt beschaffen ist. Die Wahrheit des Physikalismus ist nicht von vornherein festgelegt oder gar tautologisch. Die kontingenten Entitäten unserer Welt wie Steine, Wasser, Photonen und Neutrinos sind Wahrmacher für den Physikalismus. Unsere Welt hätte anders beschaffen sein können, sodass alle kontingenten Eigenschaften unserer Welt essentiell mental wären, und in solch einem Szenario wäre der Physikalismus falsch. Gründliche experimentelle Studien und die daraus gewonnenen Erkenntnisse führen möglicherweise zu dem Schluss, dass nicht alles in die Physik integriert werden kann. Das ist dann der Fall, wenn in einer idealen Physik bei einem beobachtbaren physikalischen Effekt keine physikalische Ursache gefunden werden kann und wir aufgrund dessen durch einen Schluss zur einzigen Erklärung zum Ergebnis kommen, dass etwas nicht-reduzierbares Mentales die Ursache war. Diese Replik zum Trivialitätsvorwurf spiegelt in diesem Punkt die obige Diskussion zur kausalen Geschlossenheit im Zusammenhang mit der Via Negativa wider. Durch Klarstellung dieser Replik kann nun ganz einfach gegen den Vorwurf der Mentalität argumentiert werden. Falls die physikalische Ontologie durch genuine mentale Entitäten erweitert werden würde, würde der Physikalismus falsch sein, weil in diesem Fall eine Form des Dualismus wahr wäre. Beispielsweise wären Qualia und Intentionalität als etwas nicht-reduzierbares Mentales Falschmacher des Physikalismus ${ }^{15}$. Im gleichen Atemzug ist auch der Vorwurf der NichtFalsifizierbarkeit zu behandeln, denn der Physikalismus ist meiner Argumentation folgend eine

${ }^{15}$ Eine andere Lösung dieses Vorwurfes stammt von Wilson (2006), die ein Verbot für fundamental Mentales für die physikalische Ontologie ausgesprochen hat. Dieser Punkt wurde von anderen aufgenommen und weiterentwickelt (Tiehen, 2016). 
kontingente These, die somit auch falsch sein kann. Wenn nun aus irgendwelchen Gründen irgendetwas Mentales in die Ontologie eingeführt werden würde, dann wäre dies ganz klar ein Falsifikator für den Physikalismus.

Die Analysen in diesem Kapitel führen nun zur Zurückweisung von Prämisse (3) in Hempels Dilemma und zu folgender Definition von "physikalisch":

(physik) Eine Entität ist genau dann physikalisch, wenn es in einer finalen Theorie über Ursachen und Wirkungen der objektiven/subjektunabhängigen Welt (i) explizit als Basisentität postuliert wird oder (ii) in einer bestimmten Relation zu den Basisentitäten steht.

In diese Definition ist all das, was in diesem Kapitel erreicht wurde, miteingeflossen. Es wird ein Bezug auf eine finale physikalische Theorie hergestellt, wobei hier der Anflug einer Zirkularität vermieden wird, indem eine physikalische Theorie als eine Theorie über Ursachen und Wirkungen der objektiven/subjektunabhängigen Welt verstanden wird. Es gilt zudem dasjenige als physikalisch, welches einerseits als fundamentale Größe bzw. als eine Basisentität in der finalen Theorie postuliert wird und andererseits in einem bestimmten Abhängigkeitsverhältnis zu den fundamentalen Größen bzw. den Basisentitäten steht. Der erste Teil dieser Definition bedeutet, dass beispielsweise Elektronen, Quarks und dergleichen als physikalisch unter der Bedingung i) gelten, falls sie explizit als Basisentität angeführt werden. Gleichzeitig wollen wir auch, dass makrophysikalische Objekte wie Bäume, Steine und dergleichen ebenfalls als physikalisch gelten, auch wenn sie nicht explizit als Basisentität angeführt werden. Das wird durch die Bedingung ii) sichergestellt. So wie durch ein bestimmtes raumzeitliches Zusammentreffen von Ziegeln eine Mauer entsteht, entstehen durch ein bestimmtes raumzeitliches Zusammentreffen der Basisentitäten weitere physikalische Objekte wie Bäume und Steine.

\subsection{Zusammenfassung}

In diesem Kapitel habe ich argumentiert, dass ein adäquates Verständnis von "physikalisch" notwendig ist, um auch die Doktrin des Physikalismus zu verstehen. Die beiden Standardoptionen, um das Physikalische zu definieren, sind die intuitionsmotivierte objektbasierte Definition und die technischere theoriebasierte Definition. Nachdem ich die objektbasierte Definition ausgeschlossen habe, wurde Hempels Dilemma expliziert, das sich gegen theoriebasierte Definitionen richtet. Bei Gültigkeit von Hempels Dilemma ist der Physikalismus falsch, falls er durch eine gegenwärtige physikalische Theorie definiert wird, oder er ist trivialerweise wahr oder er ist inhaltsleer oder er schließt fundamental mentale Entitäten 
mit ein oder er ist nicht falsifizierbar, falls er durch eine zukünftige physikalische Theorie definiert wird. Um dieses Dilemma mit seinen beiden Hörnern zu vermeiden, haben Vertreter der Via Negativa vorgeschlagen, den Begriff "physikalisch" durch "nicht-mental" zu ersetzen. Ich habe argumentiert, dass das Nicht-Mentale als ersten Schritt ein klares Verständnis des Mentalen voraussetzt. So wie es bei der objektbasierten Definition des Physikalischen der Fall war, zeigten sich auch parallele Probleme bei der objektbasierten Definition des Mentalen. Bei Betrachtung der theoriebasierten Definition des Mentalen zeigte sich, dass mentale Theorien hinreichend ähnliche Ziele und Entwicklungsgrade haben, die denen von physikalischen Theorien hinreichend ähnlich sind. Auf Basis dieser Einsichten habe ich gezeigt, dass Hempels Dilemma wieder für die Via Negativa auftaucht, dessen ursprüngliches Ziel es war, genau dieses Dilemma zu unterbinden. Wenn die Via Negativa nun doch nicht besser vom Fleck wegkommt als die Standardoptionen zur Definition des Physikalischen und der darauf aufbauenden Formulierung des Physikalismus, dann sind Physikalisten eingeladen, schlussendlich bei ihrem geliebten Vokabular zu bleiben. Die Notwendigkeit, "physikalisch" durch irgendeinen anderen Ausdruck auszutauschen, ist hiermit nicht mehr gegeben. Ich habe anschließend Gründe vorgelegt, warum der Physikalist in guter Position ist, das Physikalische über eine zukünftige, finale und ideale Physik zu definieren, und trotz alledem Hempels Dilemma nicht mehr als Argument dagegen vorgebracht werden kann. Diese Vorgangsweise sichert ein hinreichend klares Verständnis des Physikalischen und erlaubt eine weitere Formulierung des Physikalismus als eine substantielle These. 


\section{Zweiter Exkurs E2: Die Via Negativa und schwacher Mentalismus}

In diesem zweiten Exkurs möchte ich an Fußnote 11 anknüpfen und zeigen, dass Hempels Dilemma auch dann wieder auftaucht, falls ein schwacher Mentalismus anstelle eines starken Mentalismus vertreten wird. Zuerst möchte ich die Begrifflichkeiten festlegen, die beiden Versionen des Mentalismus gemein sind:

(a) "Mental" umfasst alle Erkenntnisse der psychiatrischen, psychologischen und philosophischen Wissenschaften, solange sie mit Qualia und Intentionalität beschäftigt sind.

(b) "Mentale Wissenschaften" umfasst alle Disziplinen aus denjenigen

Wissenschaften, die mit Qualia und Intentionalität beschäftigt sind.

Als letztes möchte ich den schwachen Mentalismus definieren:

(c) Der schwache Mentalismus ist die These, dass es nicht-reduzierbare mentale Entitäten gibt.

Beim schwachen Mentalismus ist der wesentliche Unterschied zum starken Mentalismus, dass zusätzlich zu mentalen Entitäten auch physikalische Entitäten existieren. Dabei ist die genaue Form der mentalistischen Position, sei es der Panpsychismus, ein Substanzdualismus oder Eigenschaftsdualismus, noch offen. Eine gewisse Spielart des schwachen Mentalismus wird häufig, eben in der einen oder anderen Form, in der gegenwärtigen Philosophie des Geistes vertreten.

Hinsichtlich der Bewertung der Ziele und der Entwicklungsgrade mentaler Theorien bleibt ebenso alles gleich zwischen einem starken oder einem schwachen Mentalismus. Es bleiben auch Ekmans (1999) Modell der Basisemotionen, Barretts (2017) Theorie der begrifflichen Akte und verschiedenste panpsychistische Ansätze (Seager, 2019) bestehen, unabhängig von der vertretenen Art des Mentalismus. Somit sind die Ziele physikalischer und mentaler Theorien dieselben, weil auch bei Gültigkeit eines schwachen Mentalismus beide Theorienbereiche immer noch auf die Ursache-Wirkung-Beziehungen unserer Welt abzielen. Ebenso sind die Entwicklungsgrade mentaler Theorien innerhalb eines schwachen Mentalismus in Hinblick auf Elaboriertheit, Überzeugungskraft und detailreichem Abdecken vieler Themenbereiche mit physikalischen Theorien vergleichbar. Mentale Theorien entwickeln sich im Laufe der Zeit, es werden neue Anwendungsbereiche geschaffen und eine Erweiterung der Themenbereiche sowie einer tiefergehenden, detailreicheren Abdeckung bestehender Themenbereiche ist im Gange. 


\section{E2.1. Hempels Dilemma und schwacher Mentalismus}

Das erste Horn von Hempels Dilemma besteht aus dem Problem, "mental" durch Bezug auf die gegenwärtigen mentalen Wissenschaften zu definieren. Mentale Theorien unterliegen laufend Revisionen, werden verändert und jegliche zukünftige Versionen mentaler Theorien würden fundamental nicht-mentale, also physikalische Entiäten, postulieren. Eine Induktion über die Wissenschaftsgeschichte macht deutlich, dass sich mentale Theorien durch die Veränderungen und Fortschritte entwickeln, was wiederum zu neuen mentalen Erkenntnissen führt. Die Konsequenz, "mental" durch irgendeine gegenwärtige mentale Theorie zu definieren, stellt die Bewertung jeglicher gegenwärtigen Theorie innerhalb der mentalen Wissenschaften als unvollendet und demnach streng genommen auch als falsch dar.

Das zweite Horn von Hempels Dilemma besteht aus dem Problem, "mental" durch Bezug auf eine vollendete, finale und ideale mentale Wissenschaft zu definieren. Eine finale Wissenschaft würde keine Revision mehr durchleben und alles in unserer mentalen Welt würde aus ihr folgen. Hier ist wichtig zu unterscheiden, dass beim schwachen Mentalismus innerhalb des mentalen Bereiches alles folgen würde, jedoch nicht alle Sachverhalte innerhalb des mentalen und physikalischen Bereiches. Der Grund ist, dass alle Sachverhalte für den physikalischen Bereich aus der vollendeten, finalen und idealen Physik folgen würde. Beim zweiten Horn dieses Dilemmas ergeben sich wiederum vier Interpretationen, diesmal etwas abgeändert im Vergleich zum starken Mentalismus. Erstens, der schwache Mentalismus würde trivialerweise wahr werden, weil alles und jedes in der goldenen Zeit der mentalen Wissenschaft auch innerhalb des mentalen Bereiches als mental gelten würde. Es würde nichts im mentalen Bereich geben, das nicht auch gleichzeitig in den mentalistischen Rahmen hineinfiele. Zweitens, der schwache Mentalismus würde ohne jeglichen spezifischen Inhalt sein, weil wir jetzt noch nicht wissen, was konkret Teil einer idealen mentalen Wissenschaft sein würde. Drittens, indem alles und jedes in die mentale Wissenschaft aufgenommen wird, bleibt auch die Gefahr übrig, dass schlussendlich ebenso etwas fundamental Physikalisches in die mentale Wissenschaft und damit in den mentalen Bereich aufgenommen wird. Viertens, wenn eine finale mentale Wissenschaft alles Mentale in unserer Welt umfassen würde, würde auch nichts diese Theorie falsifizieren können, sie wäre immun gegen jeglichen Widerlegungsversuch. Also kann eine Formulierung von Hempels Dilemma gegeben werden, die sich gegen die Via Negativa richtet $^{16}$.

${ }^{16}$ Aus denselben Gründen wie beim starken Mentalismus wird die objektbasierte Definition als möglicher Einwand gegen (1') ausgeschlossen. Die Argumentation war, dass eine intuitionsbasierte Definition des Mentalen durch Bezug auf paradigmatisch mentale Objekte aufgrund des Zirkularitätsproblems und der Unzulänglichkeit, zwischen mentalen und physikalischen Eigenschaften zu differenzieren, scheitert. 
(1') "Mental" wird entweder durch Bezug auf eine gegenwärtige mentale Theorie oder durch Bezug auf eine ideale mentale Theorie definiert.

(2') Wenn "mental" durch Bezug auf eine gegenwärtige mentale Theorie definiert wird, dann ist der schwache Mentalismus falsch.

(3') Wenn "mental" durch Bezug auf eine ideale mentale Theorie definiert wird, dann ist der schwache Mentalismus

(3a') trivialerweise wahr oder

(3b') inhaltsleer oder

(3c') beinhaltet fundamental Physikalisches oder

(3d') ist nicht falsifizierbar.

(4') Entweder ist der schwache Mentalismus falsch oder er ist trivialerweise wahr oder inhaltsleer oder er beinhaltet fundamental Physikalisches oder er ist nicht falsifizierbar.

Die Moral von Hempels Dilemma ist nun folgende. Der schwache Mentalismus ist falsch, wenn er durch einen Bezug auf eine gegenwärtige mentale Theorie definiert wird, und (a) er ist trivialerweise wahr oder (b) er ist inhaltsleer oder (c) er beinhaltet fundamental Physikalisches oder (d) er ist nicht falsifizierbar, wenn er durch einen Bezug auf eine ideale mentale Theorie definiert wird. Der Begriff "mental" in "nicht-mental" führt ebenfalls im Rahmen eines schwachen Mentalismus zu Hempels Dilemma, genauso wie es auch ursprünglich beim Begriff "physikalisch" war.

\section{E2.2. Konklusion}

Zusammenfassend ist die Argumentation in Kapitel 2 nicht davon abhängig, ob ein starker oder ein schwacher Mentalismus vertreten wird. Bei Anwendung eines schwachen Mentalismus sind die Ziele und die Entwicklungsgrade mentaler Theorien dieselben wie bei Anwendung eines starken Mentalismus. Auf diesen Erkenntnissen aufbauend kann wiederum ein Hempel'sches Dilemma für die Via Negativa bei einer theoriebasierten Definition von "mental" vorgebracht werden, auch wenn ein schwacher Mentalismus herangezogen wird. 


\section{All in all you're just another brick in the wall}

Pink Floyd (1979) 


\section{Vom Physikalischen zum Physikalismus}

Ein Ziel in diesem Kapitel ist es, eine Übersicht zu den gegenwärtigen physikalistischen Positionen zu geben und sie in den gegenseitigen Kontext zu stellen. Dabei werden die jeweiligen Kernaussagen der einzelnen Positionen beleuchtet. Als zweites Ziel werde ich zeigen, dass bei allen Positionen die Supervenienz als gemeinsamer Nenner vorhanden ist. Die Grundaussage der Supervenienz ist, dass es keine Unterschiede in einem Bereich geben kann, wenn nicht auch Unterschiede in einem anderen Bereich gegeben sind. Im selben Atemzug werde ich argumentieren, dass die Supervenienz als minimaler Physikalismus zu verstehen ist, der zumindest als eine notwendige Bedingung für jegliche physikalistische Position zu gelten hat. Das dritte Ziel betrifft Hawthornes Blocker-Problem (Hawthorne, 2002), das sich gegen die Supervenienz und damit auch gegen jegliche darauf aufbauende physikalistische Position richtet. Hawthornes Blocker-Problem behauptet, dass der Supervenienzphysikalismus nicht die Möglichkeit ausschließen kann, dass nur deshalb das Mentale auf dem Physikalischen superveniert, weil blockierende nicht-physikalische bzw. mentale Entitäten fehlen. Falls diese Blocker vorhanden wären, würde sich die Supervenienz des Mentalen nicht mehr zeigen. Ich argumentiere gegen Hawthorne und zeige, dass ein konsequentes Weiterdenken des BlockerProblems zu einem Differenzierungsproblem und zu einem Willkürproblem für BlockerSzenarien an sich führt. Diese beiden Probleme wiederum führen zum Schluss, dass Argumente gegen den Supervenienzphysikalismus, die sich auf Blocker stützen, als Gesamtes abzulehnen sind.

\subsection{Der Bauchladen des Physikalismus}

Der Physikalismus ist die These, dass alles physikalisch sei. Antworten zur Frage, was nun in dieser Definition der Begriff "alles" bedeutet und unter welchen Gegebenheiten etwas nichts anderes als etwas Physikalisches ist, gibt es viele. Zahlreiche Antworten auf diese Frage im Laufe des 20. Jahrhundert sind auch vom vorherrschenden Zeitgeist abhängig und hängen von Entwicklungen in den Naturwissenschaften einerseits sowie anderen philosophischen Richtungen andererseits ab. Die Abfolge der verschiedenen physikalistischen Positionen ist demnach auch nicht zufällig, sondern ist als Reaktion auf die vorhergehende Position zu verstehen und in den vorherrschenden Zeitgeist eingebettet.

\subsubsection{Der Behaviorismus}

Anhänger des Positivismus waren Kritiker der Metaphysik und bezeichneten metaphysische Fragen als Scheinprobleme. Als zwei der berühmtesten Anhänger des Positivismus und Mitglieder des Wiener Kreises gelten Rudolf Carnap und Otto Neurath. Beide sprachen von 
einem Physikalismus, doch beide meinten damit im Grunde keine metaphysische These. Sie und weitere Mitstreiter verstanden darunter vielmehr eine Universalsprache der Wissenschaft (Carnap, 1931; Neurath, 1931; Hempel, 1935), wobei vor allem Carnap und Hempel so weit gingen, zu behaupten, dass jeder bedeutungsvolle Ausdruck und Satz in die physikalische Sprache übersetzt werden könne. Mit dem logischen Positivismus hat sich Anfang des 20. Jahrhunderts der logische Behaviorismus entwickelt, der im Grunde die erste moderne Theorie in der Philosophie des Geistes darstellt. Eine weitere Quelle für den Behaviorismus war die rasante Entwicklung der empirischen Psychologie zu dieser Zeit, die immer mehr das beobachtbare Verhalten und bestimmte Reiz-Reaktions-Verbindungen in den Mittelpunkt rückte.

Die Grundthese des Behaviorismus in der Philosophie des Geistes, auch gleichermaßen analytischer Behaviorismus oder logischer Behaviorismus genannt, ist die Sicht auf mentale Zustände, also auch Qualia, als nichts anderes als Verhaltensdispositionen. Als Verhaltensdisposition ist beispielsweise die Eigenschaft "zerbrechlich zu sein" anzuführen, die physikalischen Dingen wie beispielsweise Glas zugeschrieben wird. Unter bestimmten Umständen, wie das Fallenlassen eines Glases auf harten Boden, wird die Eigenschaft "zerbrechlich zu sein" manifestiert, was schließlich zum Zerbrechen des Glases führt. Somit kommt dem Glas die Eigenschaft der Zerbrechlichkeit zu, die durch das objektiv beobachtbare Glasverhalten, falls es auf harten Boden trifft, manifestiert wird. Der Behaviorist erklärt nun ebenso alle mentalen Zustände in gleicher Weise. So ist Schmerz nichts anderes als die Verhaltensdisposition, bei Verletzung des eigenen Körpers mit dem Zurückziehen des betroffenen Körperteiles zu reagieren, daraufhin aufzuschreien und zu jammern. Dieses Verhalten gilt als typisches Schmerz-Verhalten und derjenige, der diese Verhaltensdisposition nicht teilt, ist auch nicht in einem schmerzvollen Zustand.

Der Behaviorismus erbt in diesem Sinne auch die Verifikationstheorie der Bedeutung des logischen Positivismus. Dieser Verifikationismus besagt, dass nur dann ein Satz sinnvoll und wahrheitswertfähig ist, wenn er entweder analytisch ist oder empirisch beobachtbar ist. Analytische Sätze sind aufgrund der Bedeutung der involvierten Wörter wahr, wie beispielsweise die Aussage "Alle Junggesellen sind unverheiratet". Hingegen sind empirische Sätze wahr, wenn die Aussage durch Beobachtungen verifiziert werden kann, wie beispielsweise die Aussage "Meine Katze hat ein schwarzes Fell". Somit sind die Aussagen "Glas ist zerbrechlich" und "Ich habe Schmerzen" dann und nur dann wahr, wenn das entsprechende typische Zerbrechlichkeitsverhalten von Glas bzw. mein typisches Schmerzverhalten explizit beobachtbar ist und jegliche Aussagen über Zerbrechlichkeit bzw. Schmerzen in Aussagen über Verhaltensbeobachtungen übersetzt werden können. Insbesondere hinsichtlich unserer mentalen Zustände ist es ein Kategorienfehler, Schmerzen in eine andere ontologische Kategorie zu geben als das beobachtbare Verhalten, und so zu tun, als ob ein Geist zusätzlich zu einem Körper existiere (Ryle, 1949). 
Kritik am Verifikationismus des logischen Positivismus und die kognitive Wende in der Psychologie ab der zweiten Hälfte des 20. Jahrhunderts führten dazu, dass der logische Behaviorismus an Glanz verlor. Es war vor allem das Problem, den Menschen als sogenannte "black box" aufzufassen, in der keine psychischen Vorgänge stattfinden, die auch nur irgendwie zur Erklärung des Verhaltens beitragen. Zudem wurde es zunehmend unplausibel, vollständige behavioristische Übersetzungen mentaler Begriffe zu geben, da jeglicher Übersetzungsversuch wiederum mentale Begriffe aufwirft (Chisholm, 1957). Darüber hinaus wurde ebenso die anfängliche Klarheit, wann Aussagen als analytisch wahr zu gelten haben und was Analytizität eigentlich bedeutet, kritisiert (Quine, 1951). Als Ergebnis spielt der Behaviorismus in der gegenwärtigen Diskussion kaum mehr eine Rolle und wurde durch andere physikalistische Positionen abgelöst, obgleich die eine oder andere Einsicht des Behaviorismus wieder in den Nachfolgepositionen aufgegriffen wurde.

\subsubsection{Die Identitätstheorie}

In der Mitte des letzten Jahrhunderts wurde der logische Behaviorismus durch die empirische psychophysische Identitätstheorie abgelöst, die durch die Arbeiten von Place (1956), Feigl (1958/1967), Smart (1959) und Armstrong (1968) große Erfolge erfuhr. Die Grundthese der Identitätstheorie ist, dass mentale und physikalische Zustände identisch sind und damit alles Mentale nichts anderes als etwas Physikalisches ist. Die Identität ist hier im strikten Sinne zu verstehen, sodass das Mentale und das Physikalische ein und dieselben Eigenschaften aufweisen und ein und dieselbe Entität sind. So wie Temperatur mit der mittleren kinetischen Energie identisch ist, so ist auch Schmerz mit einem bestimmten Erregungsmuster im Gehirn identisch. Nach P. S. Churchland (2005) bestehen die entsprechenden Theorien nebeneinander und entwickeln sich gleichzeitig, wie es beispielsweise bei psychologischen Theorien über Emotionen und neurowissenschaftlichen Theorien über Emotionen der Fall ist, aber für Identitätstheoretiker wird letztendlich die psychologische auf die neurowissenschaftliche Theorie reduziert werden. Reduktion bedeutet, dass die Makrophänomene, wie das Erleben von Schmerz, mit Mikrophänomenen, wie chemisch-physikalisch Vorgänge im Gehirn oder sogar mit molekularen Vorgängen, identifiziert werden (Bickle, 2006). Dabei werden auch die Erklärungen, Vorhersagen, Inhalte und die ontologischen Verpflichtungen der Theorie der Makrophänomene durch die Theorie der Mikrophänomene übernommen.

Der Haupteinwand gegen die Identitätsthese ist das Argument der multiplen Realisierbarkeit (Putnam, 1967), das behauptet, dass jegliche mentalen Vorgänge nicht zu jeder Zeit und bei jeder Spezies gleich realisiert werden. Schmerz ist zum einen in einer Person über verschiedene Zeitpunkte hinweg auch verschieden realisiert und zum anderen ist Schmerz wiederum bei Menschen, Affen und Tintenfischen anders realisiert. Insbesondere beim Vergleich zwischen verschiedenen Spezies wird klar, dass die neurobiologische Struktur sehr 
verschieden ist, um eine völlig übereinstimmende neuronale Aktivität bei Schmerzen zu erwarten. So argumentiert Putnam weiter, dass nicht Typen von mentalen Eigenschaft mit Typen von physikalischen Eigenschaften identisch sein können, sondern lediglich einzelne mentale und physikalische Vorkommnisse (Token), zeitpunktabhängig und speziesabhängig, identisch sein können. Tokens sind einzelne Instantiierungen des jeweiligen Typus. Der TokenIdentitätstheoretiker nimmt also die Identität von Schmerzen eines Menschen zum Zeitpunkt t mit der der neuronalen Aktivität desselben Menschen zum selben Zeitpunkt t an (siehe z.B. Davidson, 1970), wohingegen der Typen-Identitätstheoretiker die Identität von Schmerzen als Typus mit einer neuronalen Aktivität als Typus annehmen muss. Genau Letzteres wird von Putnam und seinen Anhängern verneint.

Ob die Token-Identitätstheorie ausreicht, um als eine physikalistische Theorie zu gelten, wurde in der Literatur stark bezweifelt. So argumentieren beispielsweise Pereboom und Kornblith (1991), dass eine Reduktion des Mentalen auf das Physikalische auf Erklärungen und Theorien abzielt sowie Erklärungen und Theorien wiederum über Typen quantifizieren, nicht Tokens. Deshalb ist dem Token-Identitätstheoretiker der Startplatz im Rennen nach der besten physikalistischen Theorie bereits genommen. Eine andere Erkenntnis bringt MacPherson (2000) und argumentiert, dass eine Identität zwischen mentalen und physikalischen Tokens noch keine Aussage über deren Typen erlaubt. Es folgt somit, dass auf Typenebene kein notwendiger Zusammenhang bestehen muss. Durch dieses Fehlen eines notwendigen Zusammenhanges ist es möglich, dass etwas Mentales auch ohne etwas Physikalisches auf Typenebene auftritt und es würde beispielsweise ein komplettes physikalisches Duplikat von mir nicht dasselbe mentale Leben wie ich haben. Der Token-Identitätstheoretiker kann diese Möglichkeit nicht ausräumen und seine Version der Identitätsthese ist mit der Wahrheit eines Dualismus auf Typenebene vereinbar, doch sie sollte nicht damit vereinbar sein. In dieselbe Richtung wie MacPherson gehen Block und Fodor (1972), die betonen, dass durch eine Token-Identität noch nichts über deren Typen ausgesagt wurde (Fußnote 2, S. 159):

If physicalism is the doctrine that psychological states are physical states, then we get two versions depending whether we take "states" to refer to types or to tokens. The latter construal yields a weaker theory assuming that a token of type $x$ may be identical to a token of type $y$ even though $x$ and $y$ are distinct types.

Diese und andere Gründe haben viele davon abgehalten, eine Version eines TokenPhysikalismus zu vertreten. Für Kim (2012) ist es das Fehlen einer klaren und verständlichen Version eines Token-Physikalismus, die inn entweder mit einem Dualismus vereinbar macht oder ihn zu einen Typen-Physikalismus macht. Jedenfalls sieht Kim keine Möglichkeit einer gangbaren Version eines eigenständigen Token-Physikalismus. Der springende Punkt für inn ist, 
dass jede Version einer ernstzunehmenden physikalistischen Position, in welcher Form auch immer, ihren Fokus auf mentale und physikalische Typen legen soll. Dabei muss der Physikalist im Falle der Identitätstheorie erklären, wie diese beiden Typen identisch sein können.

\subsubsection{Der Eliminativismus}

Eine Strömung als Reaktion auf die Identitätstheorie ist der Eliminativismus bzw. der eliminative Physikalismus, der im Prinzip eine Außenseiterrolle in der Philosophie des Geistes einnimmt. Klassische Vertreter sind Feyerabend (1963) und Rorty $(1965,1970)$, wobei gegenwärtige Vertreter sich vor allen auf P. S. Churchland (z.B. 1989), P. M. Churchland (z.B. 1981) und Dennett (z.B. 1988) beschränken. Die Grundthese der Eliminativisten ist, dass mentale Zustände, so wie wir sie in unserem Vokabular und in unseren Theorien beschreiben, gar nicht existieren, und sie deshalb von unserer ontologischen Bildfläche eliminiert werden müssen ${ }^{17}$. Es wird nicht bloß Mentales auf Physikalisches reduziert, denn wenn wir bei einer finalen, vollendeten physikalischen Theorie angelangt sind, löst sich das Problem der Qualia auf. Es gibt dann nichts mehr auf der vermeintlich mentalen Seite des Identitätszeichens, das überhaupt auf das Physikalische reduziert werden könnte. All unsere alltagspsychologischen Ausdrücke wie "Wunsch", "Wille", "Schmerz" oder "Liebe" würden obsolet sein und sich auf nichts mehr beziehen. Ohne Referenz des Begriffes existiert auch nicht dasjenige, welches dieser Begriff herauspicken soll. Eine finale physikalische Theorie ersetzt unsere Theorie der Alltagspsychologie (P. S. Churchland, 1995, S. 467):

\section{Die Möglichkeit einer nichttrivialen Revision oder gar einer Ersetzung bestehender Beschreibungen höherer Ebenen durch 'neurologische harmonisierte' Kategorien höherer Ebenen ist es, die den eliminativen Materialismus als eliminativ kennzeichnet.}

Der Begriff der Alltagspsychologie und der Umstand wie sich die Alltagspsychologie entwickeln wird, ist wesentlich für den Eliminativismus. Unter Alltagspsychologie versteht man diejenige Theorie, der wir uns alle bedienen, um Sinn aus dem eigenen Handeln und dem Handeln der anderen zu gewinnen. Hierfür verwenden wir unser mentalistisches Vokabular wie "Wunsch", "Wille", "Schmerz" oder "Liebe". Der Eliminativist muss die Alltagspsychologie als eine Theorie verstehen, die sich seit der Antike nicht wesentlich weiterentwickelt hat (P. M. Churchland, 1981) und schlichtweg keine Widerspiegelung in einer finalen physikalischen Theorie erleben wird. Sie

\footnotetext{
17 Siehe jedoch die Spielart des Eliminativismus von Poslajko (2019), der darunter explizit keine ontologische These versteht, sondern eine linguistische These. Sein linguistischer Eliminativismus läuft zur normativen Forderung hinaus, dass wir das mentale Vokabular nicht mehr verwenden sollen. Diese Version eines Eliminativismus ist jedoch nicht hilfreich in der gegenwärtigen Debatte, in der es primär um die ontologische Frage geht, in welcher Art und Weise das Mentale existiert. Ein reiner linguistischer Eliminativismus ohne ontologische Konsequenzen ist deshalb für das Ziel der vorliegenden Arbeit nicht hilfreich.
} 
ist im Grunde eine radikal falsche Theorie, entweder insgesamt oder in bestimmten Teilen, ohne adäquate Erklärungen und Vorhersagen. So wie wir jetzt die Existenz von Dämonen, Hexen oder einem Phlogiston leugnen, wird in der Zukunft die Existenz von jeglichen mentalen Phänomenen geleugnet werden, weil der naturwissenschaftliche Fortschritt Einzug hält.

Der Eliminativismus wurde in der Literatur stark kritisiert, angefangen von Vorwürfen der Selbstwiderlegung, weil auch Eliminativisten zumindest an ihren Standpunkt glauben müssen (z.B. Boghossian, 1990), bis hin zu sehr technischen Einwänden bezüglich der Notwendigkeit, eine problematische Referenztheorie von leeren Begriffen anzunehmen (Stich, 1996). In diesem Zusammenhang möchte ich auf mein zweites Kapitel verweisen, in dem ich gezeigt haben, dass mentale Theorien sehr wohl mit physikalischen Theorien vergleichbar sind, zumindest hinsichtlich der Ziele und der Entwicklungsgrade. Zwar könnte der Eliminativist einwenden, dass es im Wesentlichen um die Erklärungskraft und Vorhersagekraft geht, doch mindert dies keineswegs die Errungenschaften von mentalen Theorien der vorigen beiden Punkte im Sinne ihrer Ziele und Entwicklungsgrade. Dadurch wird die allzu leicht angenommene radikale Falschheit der mentalen Wissenschaften bzw. der Alltagspsychologie infrage gestellt.

Der Eliminativist könnte jedoch als nächstes entgegenhalten, dass wir die wissenschaftliche Revolution unterschätzen und nur durch das momentane mentale Vokabular nicht glauben, dass die Alltagspsychologie falsch ist. Dieselbe Argumentation kann auch gegen meine Analyse gerichtet werden, dass die Ziele und Entwicklungsgrade mentaler und physikalischer Theorien vergleichbar sind, denn das seien sie nur deshalb, weil wir das momentane mentale Vokabular verwenden. Die Illusion des Mentalen würde somit auch für die mentalen Begriffe, kontra Stich, keine maßgeblich schwierigere Analyse nach sich ziehen als Begriffe wie Weihnachtsmann, Pegasus und alle anderen Begriffe, die sich ebenso auf nichts Reales beziehen. Was ich damit sagen will, ist, dass der Eliminativismus nicht so einfach vom Tisch geschoben werden kann und diese äußerst radikale Form des Physikalismus sich doch nicht so schlecht schlägt, wenn es um eine adäquate Bewusstseinstheorie geht.

\subsubsection{Der Funktionalismus}

Eine weitere Reaktion auf die Identitätstheorie stellt der Funktionalismus dar, der sowohl durch das Argument der multiplen Realisierbarkeit als auch durch den damaligen Technisierungsfortschritt und die vielen maßgeblichen Entwicklungen in der Computerbranche zahlreiche Anhänger gefunden hat. Alles begann mit Putnam (1967) und Fodor (1974), die den Grundstein für den Funktionalismus legten. Die anfängliche Strömung des Funktionalismus wurde vor allem in der Arbeit von Block gut dargestellt und anschließend kritisiert (v.a. Block, 1978, 1980). Gegenwärtig zählt der Funktionalismus viele Anhänger und wird laufend weiterentwickelt. Auch die möglichen Spielarten wurden stark aufgefächert. 
Die Grundthese des Funktionalismus ist die, dass alles Mentale nichts anderes als etwas Funktionales ist. Die Charakterisierung des vermeintlich Mentalen geschieht hier über die funktionale Rolle, die durch Inputs und Outputs gekennzeichnet ist. Die Funktion von Schmerz ist demnach, die Hand von der heißen Herdplatte zu nehmen, eine Gewebeverletzung der Hand zu vermeiden und dergleichen. Je nach Interpretation dieses funktionalen Zustandes geschieht dessen Realisierung in Gehirnen mit Neuronen, in Robotern mit Siliziumchips oder in irgendeinem anderen Ding mit irgendeiner anderen physikalischen Aufmachung. Der Funktionalismus ist im Allgemeinen neutral zur Frage, worin das Mentale realisiert wird. Bei Identifikation des mentalen Zustandes als Typus mit einem funktionalen Zustand des Gehirns als Typus ist der Funktionalismus mit der psychophysischen Identitätstheorie vereinbar. So waren beispielsweise Smart (1959), Lewis (1966) und Armstrong (1968) selbst "funktionalistische Typen-Identitätstheoretiker" und sahen die funktionale Rolle der Gehirnprozesse als das Ausschlaggebende $a{ }^{18}$. Nachfolgend verstehe ich den Funktionalismus immer als eine Spielart des Physikalismus und damit als eine Position, die unvereinbar mit dem Dualismus ist.

Eine weitere Aufspaltung des Funktionalismus stellt den funktionalen Zustand dar, auf den schlussendlich reduziert wird. In der Literatur findet sich das Lager des RealisiererFunktionalismus (realizer functionalism) und des Rollen-Funktionalismus (role functionalism) ${ }^{19}$. Levin (2018, Kapitel 3.4) führt die Unterscheidung so ein:

As noted earlier, if in humans this functional role is played by $\mathrm{C}$-fiber stimulation, then, according to this functionalist theory, humans can be in pain simply by undergoing $\mathrm{C}$-fiber stimulation. But there is a further question to be answered, namely, what is the property of pain itself? Is it the higher-level relational property of being in some state or other that plays the "pain role" in the theory, or the $\mathrm{C}$-fiber stimulation that actually plays this role? Role functionalists identify pain with that higher-level relational property. Realizer functionalists, however, take a functional theory merely to provide definite descriptions of whichever lower-level properties satisfy the functional characterizations.

Wenn der Standpunkt vertreten wird, dass Schmerz nichts anderes als die neuronale Eigenschaft $\mathrm{N}$ ist, welche die Schmerz-Rolle einnimmt, dann handelt es sich um einen Realisierer-Funktionalismus. Wenn im Vergleich dazu der Standpunkt vertreten wird, dass

\footnotetext{
${ }^{18}$ Block (1978) bezeichnet diese Eingrenzung auf Neuronen in Gehirnen als chauvinistisch.

${ }^{19}$ Eine weitere Position innerhalb des Funktionalismus ist die, Shoemakers Vorschlag zu folgen und unter einem funktionalen Zustand einen kausalen Zustand zu verstehen, bei dem die überlappende Menge der kausalen Kräfte zwischen der zu realiserenden Eigenschaft und der realisierenden Eigenschaft ausschlaggebend ist (Shoemaker, 2007).
} 
Schmerz nichts anderes als ein Zustand ist, die neuronale Eigenschaft $\mathrm{N}$ zu haben, welche die Schmerz-Rolle einnimmt, dann handelt es sich um einen Rollen-Funktionalismus.

Ich finde die Bezeichnungen "Realisierer"- und "Rollen"-Funktionalismus etwas unglücklich gewählt, weil in beiden Lagern die funktionale Rolle das wesentlichste Merkmal ist und jede funktionale Rolle auch wiederum realisiert wird. Aus diesem Grunde möchte ich die Bezeichnung "Realisierer-Funktionalismus" mit "Funktionalismus erster Ordnung" ersetzen und die Bezeichnung "Rollen-Funktionalismus" mit "Funktionalismus zweiter Ordnung" ersetzen und anhand dessen den genauen Unterschied erläutern. David Lewis ist beispielsweise ein Funktionalist erster Ordnung und seine Position kann folgendermaßen rekonstruiert werden (Lewis, 1966):

(1) Schmerz erfüllt die kausale Rolle $X Y Z$

(2) Der Gehirnzustand B erfüllt die kausale Rolle XYZ

(K) Schmerz = Gehirnzustand B

Für Lewis ist die Eigenschaft, Schmerzen zu haben, nichts anderes als die Eigenschaft, in Gehirnzustand B zu sein. Er ist deshalb ein Funktionalist, weil sowohl der Schmerz als auch der Gehirnzustand B dieselbe kausale Rolle einnehmen. Die kausale Rolle besteht beispielsweise aus dem Wegziehen der Hand bei einer Gewebsschädigung, falls ich auf eine heiße Herdplatte greife. Das Wissen, dass Schmerzen diese kausale Rolle einnehmen, stammt aus einer Begriffsanalyse. Das Wissen, dass der Gehirnzustand B dieselbe kausale Rolle einnimmt, stammt aus den Erkenntnissen der Naturwissenschaften.

Im Kontrast dazu lässt sich ein Funktionalismus zweiter Ordnung wie folgt rekonstruieren (z.B. Melnyk, 2003):

(1') Schmerz erfüllt die kausale Rolle XYZ

(2') Der Zustand Z erfüllt die kausale Rolle XYZ

(K1) Schmerz = Zustand Z

Für Vertreter eines Funktionalismus zweiter Ordnung ist die Eigenschaft, Schmerzen zu haben, nichts anderes als die Eigenschaft, in Zustand $Z$ zu sein. Hier nehmen Schmerzen und der Zustand Z dieselbe kausale Rolle ein. Die kausale Rolle besteht auch hier darin, die Hand bei einer Gewebsschädigung von der heißen Herdplatte wegzuziehen. Das Wissen, dass Schmerzen diese kausale Rolle einnehmen, stammt aus einer Begriffsanalyse. Das Wissen, dass der Zustand Z dieselbe kausale Rolle einnimmt, entspricht der Forderung des Funktionalismus, eine Reduktionsbasis für Schmerzen bereitzustellen, die nicht das biologische Substrat eines Gehirnzustandes oder dergleichen darstellt. 
Was passiert jedoch, wenn unsere naturwissenschaftlichen Erkenntnisse zeigen, dass nun auch der Gehirnzustand B dieselbe kausale Rolle wie der Zustand Z einnimmt? Ein Vertreter eines Funktionalismus zweiter Ordnung müsste eine weitere Prämisse aufnehmen und zu einer weiteren Konklusion kommen:

(3') Gehirnzustand B = Zustand Z

(K2) Schmerz $=$ Gehirnzustand B

Der Zustand Z war in diesem Fall eher als ein Platzhalter zu verstehen, bis der tatsächliche physikalistische Realisierer gefunden wird. Schlussendlich wird auf Basis der naturwissenschaftlichen Erkenntnisse der Gehirnzustand B identifiziert, der dieselbe kausale Rolle wie der Zustand Z einnimmt. Damit wären wir durch die Transitivität der Identität aus (K1) und ( $3^{\prime}$ ) bei der endgültigen Konklusion (K2) angelangt. Natürlich könnte ein Vertreter eines Funktionalismus zweiter Ordnung die Aufnahme von Prämisse (3') und damit auch die Konklusion (K2) verhindern, indem er auf seinem Punkt beharrt, dass Schmerzen eben nicht auf das biologische Substrat reduziert werden können, weil es auf den Zustand Z ankommt. Ich denke nicht, dass dies ein gangbarer Weg wäre, weil es der naturwissenschaftlichen Haltung des Funktionalisten entgegenstehen würde. Der Beweggrund eines jeden Funktionalisten ist es ja gerade, eine naturwissenschaftliche Erklärung der Abhängigkeit zwischen dem Mentalen und dem Physikalischen zu liefern. Wenn also unsere naturwissenschaftlichen Erkenntnisse auf Prämisse (3') hindeuten, dann sehe ich keinen guten Grund, Prämisse (3') zu leugnen.

Der springende Punkt des Funktionalismus ist gerade die Akzeptanz der multiplen Realisierbarkeit, weshalb die Identitätsaussagen beider funktionalistischer Spielarten eine Token-Identität sein müssten. Der Rückzug auf funktionalistische Token-Identitäten erbt jedoch dieselben Probleme, wie sie in der Diskussion der Identitätsthese aufgetaucht sind. TokenIdentitäten sagen nichts über die dazugehörigen Typen aus, weshalb jegliche TokenIdentitätstheorie mit einem Dualismus auf Typenebene vereinbar ist. Eine adäquate Bewusstseinstheorie hat jedoch auch die Frage der Abhängigkeit der Typen zueinander zu klären (Kim, 2012). Diese Forderung kommt der funktionalistische Token-Identitätstheoretiker nicht nach.

\subsubsection{Der Repräsentationalismus}

Ab den 90er-Jahren und dem Beginn der 2000er-Jahre hat sich der physikalistisch orientierte Repräsentationalismus bzw. der Intentionalismus entwickelt, begonnen bei Tye (1995), Dretske (1995) und Crane (1998) bis hin zu Carruthers (2000) und Levine (2003). Die Grundthese des Repräsentationalismus ist die, dass alles Mentale nichts anderes als der Inhalt einer Repräsentation ist und Repräsentationen wiederum nichts anderes als bestimmte physikalische 
Vorgänge sind. An dieser Stelle ist Intentionalität im Verständnis dieses Ansatzes wesentlich, denn in einer anderen Formulierung behauptet der Repräsentationalismus, dass qualitative Zustände nichts anderes als intentionale Zustände sind. Das Schmerzerlebnis ist nichts anderes als das Gerichtetsein auf dasjenige, was der Schmerz als Inhalt hat. Die größte Motivation, eine repräsentationalistische Bewusstseinstheorie zu vertreten, kommt vom Argument der Transparenz von Erlebnissen (Harman, 1990). Es besagt, dass durch Introspektion die intrinsische Natur von Erlebnissen, in unserem Beispiel das Schmerz-Quale, nicht wahrgenommen werden kann, sondern man durch das Erlebnis "hindurchsieht". Dadurch kann lediglich das Objekt, auf das sich das Erlebnis bezieht, wahrgenommen werden. So wie auch bei den anderen Bewusstseinstheorien kommt der Repräsentationalismus in verschiedenen Spielarten daher. Um die einzelnen Positionen gruppieren zu können, folge ich Lycans Ansatz und unterscheide einerseits zwischen einem starken oder schwachen Repräsentationalismus und andererseits zwischen einem engen oder weiten Repräsentationalismus (Lycan, 2019, Kapitel 2.1 und 2.2).

Der starke Repräsentationalismus verteidigt die These, dass Qualia auf Intentionalität reduziert werden können oder dass Qualia durch Intentionalität vollständig konstituiert sind. Hingegen behauptet der schwache Repräsentationalismus, dass Qualia durch Intentionalität mitkonstituiert sind und neben Intentionalität noch einen ungerichteten, subjektiven Erlebnisgehalt zulassen. Als gangbare Bewusstseinstheorie eignet sich nur ein starker Repräsentationalismus, weil die Kernaussage des schwaches Repräsentationalismus unproblematisch ist. In einer sehr liberalen Auffassung ist jede Stimmung, jedes Gefühl zumindest auf sich selbst gerichtet. Nachfolgend werde ich unter einem Repräsentationalismus den philosophisch interessanten starken Repräsentationalismus verstehen.

Ein enger Repräsentationalismus behauptet, dass Intentionalität ausschließlich von Vorgängen innerhalb der Person abhängt und unabhängig von Umgebungsfaktoren ist. Es sind unter dieser Sichtweise Gehirnprozesse alleine, die für das Gerichtetsein ausschlaggebend sind (z.B. Levine, 2003). Wird hingegen ein weiter Repräsentationalismus vertreten, dann ist Intentionalität auch von Vorgängen außerhalb der Person und bestimmten Umgebungsfaktoren abhängig (z.B. Tye, 1995). Letzteres stützt sich durchgehend auf Putnams Gedankenexperiment der Zwillingserde, in dem die Person auf der Erde die Überzeugung hat, dass Wasser $\mathrm{H}_{2} \mathrm{O}$ sei, jedoch eine physikalisch identische Person auf der Zwillingserde die Überzeugung hat, dass Wasser XYZ sei (Putnam, 1975). Obwohl beide physikalisch identischen Personen Überzeugungen hinsichtlich Wasser haben, ist der Inhalt deren Gedanken verschieden, nämlich $\mathrm{H}_{2} \mathrm{O}$ auf der Erde und $\mathrm{XYZ}$ auf der Zwillingserde. An diesem Gedankenexperiment ließe sich zeigen, dass Umgebungsfaktoren einen Einfluss auf den Inhalt mentaler Vorgänge haben ${ }^{20}$.

${ }^{20}$ Putnam (1975, S. 227) hat den Slogan geprägt, dass Bedeutung einfach nicht im Kopf sei ("meaning just ain't in the head"). 
Das größte Problem des Repräsentationalismus ist, dass das ursprüngliche Problem, nämlich eine physikalistische Lösung des Bewusstseinsproblems zu finden, lediglich auf die Seite der Intentionalität gewechselt ist. Falls nun Qualia tatsächlich auf intentionale Zustände reduziert werden können oder sie völlig durch intentionale Zustände konstituiert sind, sagt das noch nichts darüber aus, ob nun auch intentionale Zustände physikalisch sind. Das Ausgangsproblem wurde lediglich an eine andere Stelle verschoben. Denselben Punkt macht Alter (2007) in seiner Diskussion des Repräsentationalismus (S. 74): "Then instead of asking, 'Is phenomenally conscious experience physical?' we should ask, 'Is phenomenal representation physical?' But the latter question raises the same issues as the former."

\subsubsection{Der Fundierungsphysikalismus}

In den 2000er-Jahren hat sich der Fundierungsphysikalismus (grounding physicalism) entwickelt, dessen Anhänger eine neue, fundamentale Relation zwischen dem Mentalen und dem Physikalischen favorisieren. Vertreter wie Fine (2001), Schaffer (2009), Rosen (2010) und Dasgupta (2014) argumentieren, dass diese neue Relation eine weitere Erklärung neben den klassischen, wissenschaftlichen Erklärungen darstellt, bei der auch das Erklärende mit dem zu Erklärenden in fundamentaler Art und Weise verbunden ist. Diese neue Relation ist die Fundierungsrelation, die metaphysisch das Auftreten des Mentalen bei Vorhandensein des Physikalischen sichert. Das Wesentliche für Fundierungsphysikalisten ist nicht die Frage, ob etwas Mentales existiert, in welcher Art auch immer, sondern wie es existiert. Die Grundthese des Fundierungsphysikalismus ist, dass alles Mentale im Physikalischen fundiert ist.

Der Fundierungsphysikalismus hat bereits eine scharfe Kritik erfahren, in der die Motivation, diese Spielart zu vertreten, angriffen wird, ebenso wie viele grundlegende Inhalte (siehe vor allem Wilson, 2014, 2016 und Melnyk, 2016). Das größte Problem für den Fundierungsphysikalismus sehe ich jedoch darin, dass nicht klar ist, was der wesentliche Beitrag einer neuen, primitiven Relation ist, was die anderen bisher bekannten Relationen (d.h. behavioristische, identitätstheoretische, eliminativistische, funktionalistische und repräsentationalistische) nicht bringen. Der Preis der Einführung der Fundierungsrelation ist eine Postulierung einer weiteren grundlegenden Relation, die jedoch nichts anderes sagt, als dass das Mentale im Physikalischen fundiert ist. Der Fundierungsphysikalismus liefert keine Antworten, warum diese Relation der Fundierung besteht, und eine Beantwortung der WarumFrage der Fundierung würde auf einen schlichtweg zu akzeptierenden Sachverhalt hinauslaufen, da etwas Fundamentales nicht weiter erklärt werden muss.

Der Fundierungsphysikalismus liefert auch keine Antworten, wie die Relation der Fundierung nun genau etwas Mentales zu etwas Physikalisches macht. Entweder müsste behauptet werden, dass es schlichtweg der Fall ist und die Fundierungsrelation an sich die physikalische Natur sichert, oder die Fundierungsrelation müsste um eine weitere Relation 
ergänzt werden wie beispielsweise einer Identitätsrelation. Im Falle einer Identität des Mentalen mit dem Physikalischen wäre erklärt, warum durch die Fundierungsrelation das vermeintlich Mentale eigentlich etwas Physikalisches ist. Doch dann wäre auch die Postulierung einer Fundierungsrelation unnötig, weil die Identitätsrelation bereits hinreichend wäre. Somit bedient sich der Fundierungsphysikalist entweder einer bisher etablierten physikalistischen Relation zwischen dem Mentalen und dem Physikalischen, oder er bedient sich bei der Beantwortung dieser Fragen der Feststellung, dass es eben so sei, weil es sich um einen neuen, primitiven Sachverhalt handle, der zu akzeptieren sei. Ich weiß nicht, welche Option die bessere darstellt, doch ich bin überzeugt, dass keine der beiden Optionen dem Fundierungsphysikalismus einen Startvorteil bringen.

\subsection{Die Supervenienz als minimaler Physikalismus}

Es ist an diesem Punkt offensichtlich, dass im Lager der Physikalisten viele verschiedene Spielarten vorhanden sind und mehr oder weniger verschiedene Interpretationen der Relation zwischen dem Mentalen und dem Physikalischen Anwendung finden. Was jedoch allen Ansätzen gemeinsam ist, ist zumindest die Annahme einer Supervenienzrelation mit der Kernaussage, dass es keine Veränderung im Mentalen gibt ohne auch eine Veränderung im Physikalischen. Verbal näher spezifiziert bedeutet dies, dass es keine Veränderung im Mentalen gibt ohne auch eine Veränderung in der neuronalen Basis (Identitätstheorie) oder in den kausalen Rollen (Funktionalismus) oder im Verhalten (Behaviorismus) oder in der Fundierungsbasis (Fundierungsphysikalismus) oder in der Repräsentationsbasis (Repräsentationalismus) oder irgendeiner anderen Relation zwischen dem Mentalen und dem Physikalischen, die dem Mentalen einen sicheren Platz in der Ontologie des Physikalisten sichert. Ich bleibe nachfolgend bei der allgemeinen Formulierung des Begriffes "physikalisch", ohne weiter auf das Vokabular der verschiedenen Spielarten des Physikalismus einzugehen. Das Gesagte kann bei Bedarf getrost mutatis mutandis auf die jeweiligen physikalistischen Positionen in deren jeweilig spezifisches Vokabular übertragen werden.

Im Falle des Eliminativismus scheint es dem ersten Anschein nach eine Supervenienzrelation zwischen dem Mentalen und dem Physikalischen nicht zu geben. Man könnte glauben, dass eine Supervenienz erst gar nicht möglich sei, weil Eliminativisten behaupten, es gebe nichts Mentales. Ich möchte hier gleich wie Stoljar (2015, Kapitel 5) argumentieren, dass auch im Eliminativismus eine Supervenienzrelation gegeben ist. Der Grund ist, dass es auch hier keine Veränderung des Mentalen gibt, ohne auch eine Veränderung im Physikalischen. Nur ist hier der spezielle Fall gegeben, dass es überhaupt nichts Mentales gibt, dennoch ist auch hier die Kernthese der Supervenienz erfüllt. 
Das Ablehnen der Supervenienz ist bedeutungsgleich mit dem Ablehnen des Physikalismus (Kim, 1984) und der wesentlichste Grund dafür ist der folgende ${ }^{21}$. Die Supervenienzrelation sagt auch, dass es nur dann eine Änderung im Mentalen gibt, wenn es auch eine Änderung im Physikalischen gibt. Wenn nun mein Angsterlebnis einem Glücksgefühl weicht, dann haben sich nicht nur diese supervenierenden Eigenschaften geändert, sondern auch gleichzeitig die Supervenienzbasis dieser Eigenschaften. Falls sich jedoch meine Erlebnisqualität ändert und die vermeintliche Supervenienzbasis gleich bleibt, dann ist etwas anderes für die Änderung der Erlebnisqualität verantwortlich und es existiert keine Gesetzmäßigkeit zwischen der Supervenienzbasis und den supervenierenden Eigenschaften. Dieses "Andere" muss nun außerhalb der Supervenienzbasis liegen und nicht-physikalisch sein, weil es entweder die Erlebnisqualität selbst ist, die der Supervenienzgeschichte entflieht, oder eine Eigenschaft, ein Objekt, eine Substanz oder dergleichen ist, was die Relation zur Erlebnisqualität nicht-physikalisch macht. In jedem Falle lässt es den Schluss zu, dass die Supervenienzbasis weder notwendig noch hinreichend für eine bestimmte Erlebnisqualität ist, sondern bestenfalls mit wechselnden mentalen Entitäten assoziiert ist. Schlechtestenfalls ist das Fehlen einer Supervenienzrelation auch mit dem kompletten Fehlen einer Erlebnisqualität vereinbar (siehe Chalmers' Zombie-Argument in Chalmers, 1996). Alle mentalen Dinge, Objekte, Eigenschaften und dergleichen, die sich trotz Vorhandensein einer vermeintlichen

Supervenienzbasis nach Belieben abwechseln, falls sie sich überhaupt zeigen, begründen einen Dualismus.

Für die Formulierung des minimalen Physikalismus brauchen wir noch weitere, teilweise auch technische, Überlegungen. Behauptungen des Physikalismus sollen sowohl innerhalb jedes widerspruchsfreien Szenarios als auch zwischen verschiedenen widerspruchsfreien Szenarien gelten. Widerspruchsfreie Szenarien sind solche, in denen es keine logischen Widersprüche gibt. So ist ein Szenario widerspruchsfrei, wenn es nur Menschen mit einer Körpergröße über 2 Meter gibt oder alle Menschen nur noch in Europa leben würden. Im Kontrast dazu ist ein Szenario widersprüchlich, wenn es darin runde Dreiecke gibt oder verheiratete Junggesellen. Verschiedene Szenarien als Ausdrücke dafür, wie es hätte sein können, werden als mögliche Welten verstanden. Mit einer möglichen Welt meine ich also ein widerspruchsfreies Szenario, in dem bestimmte Gegebenheiten vorliegen können. Eine mögliche Welt kennen wir bereits, es ist die aktuale Welt $W$, in der wir leben.

Konkrete Versionen der Supervenienzthese gibt es viele und es lässt sich demnach die Supervenienzthese auch in vielen Weisen entwickeln (siehe für eine Übersicht Wilson, 2005). Ich möchte mich in dieser Arbeit auf die Grundüberlegungen beziehen und beschränke mich auf die häufigsten Formulierungen. Diese Grundüberlegungen betreffen zum einen die Gültigkeit der Supervenienz, nämlich innerhalb einer möglichen Welt oder über verschiedene mögliche Welten

21 Siehe als Gegenposition Montero (2013), die argumentiert, dass der Physikalismus keine Supervenienz implizieren muss und damit die Falschheit der Supervenienzthese mit der Wahrheit des Physikalismus vereinbar ist. 
hinweg, und zum anderen, ob über Individuen oder über mögliche Welten formuliert werden soll. Die Supervenienzthese kann nun für Individuen in einer möglichen Welt gelten, in der es eine bestimmte Aufmachung im A-Bereich (A-Aufmachung) und eine bestimmte Aufmachung im B-Bereich (B-Aufmachung) gibt. Nehmen wir weiters auch zwei Individuen $X$ und $Y$ an. Hier umfasst ex hypothesi die A-Aufmachung alle mentalen Gegebenheiten und die B-Aufmachung alle physikalischen Gegebenheiten. Kim (1984) nennt dies schwache Supervenienz ${ }^{22 .}$

(Sschwach) A superveniert in einer schwachen Art und Weise auf B genau dann, wenn notwendigerweise für $X$ und $Y$ gilt: Wenn $X$ und $Y$ dieselbe $B-$ Aufmachung haben, dann haben $X$ und $Y$ auch dieselbe A-Aufmachung.

Die Supervenienzthese kann aber auch stärker aufgefasst werden und für Individuen über alle möglichen Welten hinweg gelten. Diese Version nennt Kim (1984) starke Supervenienz ${ }^{23}$ :

(Sstark) A superveniert in einer starken Art und Weise auf B genau dann, wenn notwendigerweise für $X$ und jeder Eigenschaft $F$ der $A$-Aufmachung gilt: Wenn $X$ die Eigenschaft $F$ hat, dann gibt es eine Eigenschaft $G$ der $B$ Aufmachung, sodass $X$ die Eigenschaft $B$ hat und notwendigerweise, wenn $y$ die Eigenschaft $G$ hat, auch die Eigenschaft $F$ hat.

(Sstark) impliziert (Sschwach), denn wenn über alle möglichen Welten hinweg die AAufmachung zwischen den Individuen $X$ und $Y$ gleich bleibt, dann bleibt es auch innerhalb einer bestimmten Welt gleich.

Die Supervenienzthese kann anstelle von Individuen auch über ganze möglichen Welten formuliert werden. Dies nenn Kim globale Supervenienz (Kim, 1984) ${ }^{24}$.

(Sglobal) A superveniert global auf B genau dann, wenn gilt: Wenn die möglichen Welten W1 bis Wi dieselbe B-Aufmachung haben, dann haben die möglichen Welten W1 bis Wi auch dieselbe A-Aufmachung.

\footnotetext{
22 Kim (1984) bietet zwei äquivalente Formulierungen der schwachen Supervenienz an. Auf S. 158 schreibt Kim: "A weakly supervenes on $B$ if and only if necessarily for any $x$ and $y$ if $x$ and $y$ share all properties in $B$ then $x$ and $y$ share all properties in A - that is, indiscernibility with respect to B entails indiscernibility with respect to A". Auf S. 163 bringt Kim die folgende äquivalente Formulierung: "A weakly supervenes on $B$ if and only if necessarily for any property $F$ in $A$, if an object $x$ has $F$, then there exists a property $G$ in $B$ such that $x$ has $G$, and if any $y$ has $G$ it has $F$ ".

${ }^{23} \mathrm{Kim}$ (1984) führt die starke Supervenienz wie folgt ein (S. 165): "A strongly supervenes on B just in case, necessarily, for each $x$ and each property $F$ in $A$, if $x$ has $F$, then there is a property $G$ in $B$ such that $x$ has $G$, and necessarily if any y has $\mathrm{G}$, it has $\mathrm{F}^{\prime \prime}$.

${ }^{24}$ Kim (1984) hat die globale Supervenienz auf S. 168 eingeführt: "A globally supervenes on B just in case worlds that are indiscernible with respect to B ("B-indiscernible," for short) are also A-indiscernible".
} 
(Sstark) impliziert (Sglobal), denn wenn physikalisch ununterscheidbare Individuen über alle möglichen Welten hinweg auch mental ununterscheidbar sind, dann sind physikalisch ununterscheidbare Welten auch mental ununterscheidbar ${ }^{25}$. Ob nun auch (Sglobal) (Sstark) impliziert, ist eine viel diskutierte Sache in der Literatur (siehe v.a. Kim, 1993), doch es scheint sich herauszukristallisieren, dass (Sglobal) nicht (Sstark) impliziert. So führt beispielsweise Chalmers (1996) aus, dass plausiblerweise alle biologischen Eigenschaften global auf allen physikalischen Eigenschaften supervenieren und physikalisch ununterscheidbare Welten auch biologisch ununterscheidbar sind. Aber biologische Eigenschaften supervenieren nicht individuell auf physikalische Eigenschaften, weil physikalisch ununterscheidbare Individuen in biologischen Eigenschaften unterscheidbar sein können. Das ist deshalb der Fall, weil biologische Veränderungen durch eine Anpassung an die Umwelt geschehen kann. Die Frage einer möglichen Implikation von (Sglobal) zu (Sstark) ist eine sicherlich interessante Frage, doch die Antwort hat keinen Einfluss auf die vorliegende Arbeit. Um die Supervenienz für eine Formulierung eines minimalen Physikalismus gebrauchen zu können, sind sowohl (Sstark) als auch (Sglobal) hinreichend stark und erfüllen im ersten Moment den Zweck. Es erfüllt die Anforderung, eine beständige Relation zwischen der B-Aufmachung und der A-Aufmachung zu liefern. Die Relation geht hier so weit, dass die B-Aufmachung die Existenz der A-Aufmachung notwendig macht. Denn um Kripkes Bild zu verwenden, war alles, was Gott zu tun hatte, lediglich die B-Aufmachung zu erschaffen, und die gesamte A-Aufmachung folgt ohne weiteres Zutun Gottes (Kripke, 1980). Die These des Physikalismus kann nun wie folgt formuliert werden:

(P1) Der Physikalismus ist genau dann wahr, wenn die möglichen Welten W1 bis Wi mit komplett gleicher B-Aufmachung auch dieselbe A-Aufmachung haben.

In der gegenwärtigen Literatur hat sich eine globale Supervenienzformulierung durchgesetzt (v.a. Lewis, 1983; Chalmers, 1996; Jackson, 1998a), doch mit einer weiteren Spezifizierung aufgrund eines differenzierten Einwandes. Der Einwand ist der, dass es physikalisch ununterscheidbare Welten gibt, bei denen eine mögliche Welt zusätzliche fundamental mentale Eigenschaften, Relationen oder Substanzen beherbergt wie Seelen, Geister oder die Macht in Star Wars. Das Problem dabei ist, dass bei Wahrheit des Physikalismus solch eine Welt nicht möglich sein kann, die ein physikalischen Duplikat unserer aktualen Welt ist, jedoch zusätzlich fundamental mentale Entitäten aufweist und somit nicht dieselbe A-Aufmachung hat. Solch eine Welt ist jedoch möglich bei Anwendung von (P1). Falls diese mögliche Welt auch die aktuale Welt ist, dann wäre (Sglobal) falsch und der Physikalismus falsch. Damit ist die Supervenienz

${ }^{25}$ Kim nahm zuerst noch eine Äquivalenz zwischen er starken Supervenienz und der globalen Supervenienz an (Kim, 1984), doch lenke später ein, dass die starke Supervenienz die globale Supervenienz impliziert, aber nicht umgekehrt (Kim, 1987). 
des Mentalen auf dem Physikalischen nicht mehr gegeben und der Physikalismus wäre widerlegt. Diese Möglichkeit der Falschheit von (Sglobal) und des Physikalismus von vornherein auszuschließen, würde den Physikalismus immer definitorisch wahr machen. Mir ist niemand bekannt, der diesen Weg in der Philosophie des Geistes beschreitet. Den anderen Weg zu beschreiten, nämlich eine mögliche Welt mit fundamental mentalen Entitäten trotz gleicher B-Aufmachung zu unserer aktualen Welt ad-hoc zu verneinen, würde ein allzu mutiger und willkürlicher Zug sein. Glücklicherweise gibt es andere Möglichkeiten, auf dieses Problem zu reagieren.

Ein Ausweg ist, die Reichweite der möglichen Welten in (Sglobal) einzuschränken und nur auf bestimmte mögliche Welten anzuwenden. Der Physikalist kann erlauben, dass es in anderen möglichen Welten Seelen geben kann, nur nicht in hinreichend ähnlichen Welten zu unserer aktualen Welt. Der Physikalist kann ebenso mögliche Welten erlauben, in der es nur Seelen, Geister und Feen gibt, nur eben nicht in hinreichend ähnlichen Welten zu unserer aktualen Welt. Der wesentliche Punkt ist, dass die Wahrheit des Physikalismus nicht mit der Aktualität solcher Welten vereinbar ist, jedoch mit der bloßen Möglichkeit vereinbar sein soll26. Es muss sozusagen ein Stopp-Zeichen in (Sglobal) eingefügt werden, das wiederum in verschiedener Art und Weise entwickelt werden kann. Ich möchte nachfolgend auf den vielzitierten Vorschlag von Jackson (1998a) eingehen ${ }^{27}$. Nach Jackson ist das Wesentliche des Physikalismus, dass eine Duplizierung unserer Welt in allen physikalischen Angelegenheiten, und gleichzeitig nichts anderes mehr hinzugefügt wird, auch eine Duplizierung unserer Welt in allen Angelegenheiten ist. Ein physikalisches Duplikat unserer Welt ist somit auch ein Duplikat aller mentalen Entitäten (S. 12):

(B) Jede Welt, die ein minimales physikalisches Duplikat unserer Welt ist, ist ein Duplikat unserer Welt schlechthin.

Der Ausdruck "minimales physikalisches Duplikat" bedarf noch weiterer Erläuterungen. Nach Jacksons Ansatz (S. 13) ist eine mögliche Welt W1 genau dann ein minimales physikalisches Duplikat unserer aktualen Welt W, wenn es (a) exakt wie W in jeder physikalischen Angelegenheit ist und (b) nichts anderes enthält als das, was es unbedingt enthalten muss, um (a) gerecht zu werden. Die Konsequenz der Akzeptanz von (B) ist die, dass Physikalisten getrost die Möglichkeit von Geistern, cartesischen Seelen und vielen anderen fundamental

\footnotetext{
${ }^{26}$ Physikalisten akzeptieren typischerweise diese Forderung. Siehe jedoch Francescotti (2014), der die Kompatibilität des Physikalismus mit der bloßen Möglichkeit von fundamental mentalen Entitäten leugnet, wodurch in seinem Ansatz keine nicht-physikalischen Extras einen Platz haben.

${ }^{27}$ Chalmers (1996) bringt eine Supervenienzthese, die sich nur auf positive Entitäten bezieht, die genau dann als positiv gelten, wenn diese Entität in der möglichen Welt W gegeben ist und in jeder anderen möglichen Welt ebenso gegeben ist, die W als Teil hat. Positive Entitäten, oder auch Fakten wie sie bei Chalmers eingeführt werden, sind beispielsweise Bäume, Steine, eine Masse zu haben und dergleichen. Im Kontrast dazu sind negative Fakten alle negativen Existenzaussagen wie beispielsweise, dass es keine Feen, Geister oder dergleichen gibt.
} 
mentalen Entitäten erlauben können. Falls nun W1 solch eine Welt mit fundamental mentalen Entitäten wäre, dann wäre W1 zwar ein physikalisches Duplikat von W, aber es wäre kein minimales physikalisches Duplikat und sohin auch kein Duplikat schlechthin. Der Grund ist der, dass W1 mehr mentale Entitäten beherbergt als W, nämlich Geister und cartesische Seelen. Auf Basis von (B) lässt sich die These des Physikalismus in folgende Formulierung bringen:

(P2) Der Physikalismus ist genau dann wahr, wenn jede Welt, die ein minimales physikalisches Duplikat unserer Welt ist, ein Duplikat unserer Welt schlechthin ist.

Der Physikalismus ist falsch in jeder Geister-Welt, doch der Physikalismus kann wahr in unserer aktualen Welt sein. Die Wahrheit des Physikalismus in unserer Welt hängt davon ab, was unsere Welt tatsächlich als Existierendes beherbergt und ob es darin fundamental mentale Entitäten gibt.

\subsection{Hawthornes Blocker-Problem}

Eine Möglichkeit, um gegen die Physikalismus zu argumentieren, ist gegen die Supervenienzrelation zwischen dem Mentalen und dem Physikalischen zu argumentieren. Falls es gelingt, den Supervenienzphysikalismus zu widerlegen, wäre auch jede darauf aufbauende physikalistische Position widerlegt. Ein Argument gegen den Supervenienzphysikalismus stellt das Blocker-Problem von Hawthorne (2002) dar. Stellen wir uns eine Welt vor, in der die Relation zwischen dem Physikalischen und dem Mentalen nicht so stark ist wie es die Supervenienz fordert, sondern dass das Physikalische das Mentale in einem schwachen Sinne notwendig macht. Eine schwache Notwendigkeit des Mentalen bedeutet, dass das Mentale nicht in jedem einzelnen Fall durch das Physikalische impliziert ist. Die Implikation des Mentalen durch das Physikalische unter dieser Voraussetzung der schwachen Notwendigkeit liegt genau dann vor, wenn (1) die physikalischen Basisentitäten gegeben sind, (2) eine adäquate physikalische Relation zwischen den Basisentitäten sowie physikalischen Entitäten höherer Ordnung gegeben ist und (3) keine blockierenden Dinge, wie auch immer geartet, vorhanden sind. Im Kontrast dazu impliziert das Physikalische das Mentale in einem starken Sinne genau dann, wenn das Mentale in jedem Falle, komme was wolle, durch das Physikalische impliziert wird. Die Implikation des Mentalen durch das Physikalische unter dieser Voraussetzung der starken Notwendigkeit liegt genau dann vor, wenn (1) die physikalischen Basisentitäten gegeben sind und (2) eine adäquate physikalische Relation zwischen den Basisentitäten sowie den physikalischen Entitäten höherer Ordnung gegeben ist. Nichts anderes wird benötigt. 
Der Unterschied zwischen der schwachen und der starken Notwendigkeit ist die Einschränkung, die in (3) ausgedrückt ist. Diese Einschränkung nennt Hawthorne (2002) Blocker. Blocker sind nicht-physikalische bzw. mentale Entitäten, die verhindern, dass etwas Mentales in Existenz tritt. Wenn nun etwas Mentales (M) in Existenz tritt, dann impliziert das Physikalische (P, ausgedrückt in den Sätzen (1) und (2)) zusammen mit der Absenz von Blockern (B) das Mentale mit schwacher Notwendigkeit (SN):

(SN) M genau dann, wenn (P \& $\neg B)$

Ob nun die bloße Möglichkeit von Blockern eine Gefahr für Formulierungen des Physikalismus darstellt, bei denen eine Supervenienzformulierung als ein minimaler Physikalismus gilt, wird in der Philosophie des Geistes aktuell diskutiert. Angewandt auf Jacksons Supervenienzthese (B) führt die Möglichkeit von Blockern zu folgenden Problemen. Nehmen wir an, dass in der möglichen Welt W2 die Möglichkeit von Blockern besteht. Unter der Annahme von (SN) auf Basis von (B) ist der Physikalismus in W2 wahr, doch intuitiv sollte das nicht sein. W2 enthält nicht mehr mentale Entitäten als unsere aktuale Welt $W$ und enthält somit nicht mehr als es muss, um exakt wie $\mathrm{W}$ zu sein hinsichtlich der gesamten physikalischen Aufmachung. Als Konsequenz ist W2 ein minimales physikalisches Duplikat von W und damit auch ein Duplikat schlechthin von W. Aber, die Absenz von Blockern, die ex hypothesi vorhanden sein könnte, stellt eine schwache Notwendigkeit als starke Notwendigkeit in W2 dar. Dadurch ist der Physikalismus wahr, weil auf Basis von (B) W2 ein minimales Duplikat und damit auch ein Duplikat schlechthin von W ist. Jacksons (B) unterscheidet nicht zwischen starker Notwendigkeit und schwacher Notwendigkeit als Wolf in einem Schafspelz. Man sollte jedoch zwischen beiden Arten der Notwendigkeit unterscheiden, um die Wahrheit des Physikalismus in W und allen hinreichend ähnlichen möglichen Welten richtig bestimmen zu können. Anders formuliert, falls (B) eine adäquate Formulierung des (zumindest minimalen) Physikalismus ist, dann sollte der Physikalismus unter der Bedingung von (SN) aufgrund von (B) in W2 falsch sein, doch er ist es nicht.

Bisherige Lösungsversuche zu Hawthornes Blocker-Problem sind spärlich gesät. Ein Weg, dieses Problem zu umgehen, ist Leuenberger (2008) zu folgen und auf Basis der Gültigkeit der schwachen Notwendigkeit einen Physikalismus zu entwickeln, der schwächer als es der Supervenienzphysikalismus ist. Doch hier ist wiederum problematisch, dass die Supervenienz im Kern jeglicher physikalistischen Position liegt und dieses minimale Zugeständnis jedes Physikalisten verloren ginge. Bei Ablehnung der Supervenienz würde sich auch die mentale Aufmachung verändern, obwohl die physikalische Aufmachung dieselbe bliebe. Somit denke ich nicht, dass Leuenbergers Lösung noch als ein Physikalismus zu werten 
ist, ungeachtet der Möglichkeit, dass womöglich Leuenbergers Ansatz als dualistische Bewusstseinstheorie mehr Erfolg hat ${ }^{28}$.

Einen weiteren Lösungsansatz bietet Francescotti (2014) an. Er argumentiert gegen die Möglichkeit von Blockern, indem er überhaupt keine möglichen Welten zulässt, wie auch immer unähnlich zu unserer aktualen Welt, in der irgendwelche nicht-physikalischen Entitäten vorkommen. Wie ich in der Diskussion über das genaue Verständnis der Supervenienz gezeigt habe, ist es unnötig, bereits a priori auszuschließen, dass ein physikalisches Duplikat unserer Welt nicht auch mentale Entitäten beherbergen kann. Es jedoch a priori auszuschließen bedeutet, den Dualismus a priori auszuschließen, und der Physikalismus wäre trivialerweise wahr. Francescottis Lösung birgt damit weitreichendere Konsequenzen als lediglich die Verhinderung von Blockern und aus diesem Grunde sollte es nicht die Standardantwort auf Hawthorne sein, vor allem deshalb, weil es weitere Alternativen gibt.

Zu guter Letzt argumentiert Grindeland (2015) auf Basis von Widersprüchen im Begriff des Blockers selbst, dass Blocker gar nicht möglich sein können. Man hat entweder die Möglichkeit, Blocker als nicht-physikalische emergente Phänomene des Physikalischen anzusehen, oder man nimmt an, dass Blocker etwas genuin Mentales sind, die außerhalb unserer physikalischen Existenzebene liegen. Im ersteren Fall würden Blocker nicht durch das Physikalische impliziert sein, sondern in irgendeiner Weise daraus neu entstehen.

Problematisch dabei ist die brachiale Tatsachenherstellung, dass es ohne weitere Erklärung einfach so ist, wie es ist. Im letzteren Fall würde unwillkürlich die Frage auftauchen, wie etwas komplett Nicht-Physikalisches die Funktionen erfüllen kann, wie sie Hawthornes Blocker erfüllen. Sie wären komplett außerhalb des physikalischen Bereiches und damit wäre auch keine interaktionistische Lösung in diesem Falle gangbar. Um die Funktion zu erfüllen, müssten Blocker physikalisch sein, doch das raubt Hawthornes Blocker-Problem wiederum die Grundlage. Grindelands Lösungsansatz hat einen gewissen Charme und ich stimme damit überein, dass eine schlichtweg angenommene Emergenz unterm Strich nicht viel zur Erklärung beiträgt. Doch die Forderung, dass Blocker in keinster Weise Berührungspunkte mit der physikalischen Realität haben dürfen, ist eine zu starke Interpretation von Hawthornes Anliegen. So könnte Hawthorne kontern, dass er in seinen Überlegungen zu Blockern auch einen interaktionalistischen Dualismus annimmt, wie auch immer geartet, unter dessen Wahrheit die Blocker ihre Funktionen wahrnehmen können wie ursprünglich dargelegt. Somit bietet auch Grindeland keine überzeugende Lösung an.

Ich möchte nachfolgend drei neue Argumente gegen die Möglichkeit von Blockern bringen und zeigen, dass Hawthornes Blocker-Problem kein wirkliches Problem für den Physikalisten darstellt. Die Quintessenz dieser drei Argumente ist das Aufzeigen einerseits von

28 Siehe ebenfalls Chalmers (2010), der Leuenbergers Ansatz als nicht stark genug ansieht, um als eine Spielart des Physikalismus zu gelten. 
Willkürproblemen und andererseits von weiteren Differenzierungsproblemen, falls die Möglichkeit von Blockern ernst genommen wird.

\subsubsection{Eine Gruppe von Blockern}

Falls die Möglichkeit von Blockern akzeptiert wird, dann erscheint auch die Möglichkeit von mehreren Blockern gegeben zu sein, um (SN) noch weiter abzuschwächen. So macht erst die Summe mehrerer fehlender Blocker in Kombination mit dem Physikalischen das Mentale notwendig. Warum sollte die Summe der Blocker, deren Absenz notwendig für das Auftreten des Mentalen ist, nicht deutlich mehr als 10 sein oder mehr als 100 sein oder nicht gleich ins Unendliche gehen? Wenn nun mentale Entitäten (M) in Existenz treten, dann impliziert das Physikalische (P, ausgedrückt in den Sätzen (1) und (2)) zusammen mit der Absenz von unendlich vielen Blockern (B1 bis $B \infty$ ) das Mentale mit einer Notwendigkeit, die sehr viel schwächer ist als (SN). Womöglich wäre hierfür der Ausdruck "unendlich schwache Notwendigkeit" passend. Logisch sieht diese Art der Implikation in ihrer stärksten Form wie folgt aus ${ }^{29}$ :

(A1) M genau dann, wenn (P \& $\neg \mathrm{B} 1 \&$ \& $\mathrm{B} 2$ \& $\neg \mathrm{B} 3 \&$ \& .. \& $\neg \mathrm{B} \infty$ )

Hier ergeben sich zwei Probleme. Der ursprüngliche Punkt des Blocker-Problems war, dass Jacksons (B) nicht zwischen starker Notwendigkeit und schwacher Notwendigkeit als Wolf in einem Schafspelz unterscheidet. Dasselbe Problem ergibt sich hier jedoch wieder. Denn nehmen wir nun eine schwache Notwendigkeit zwischen $\mathrm{P}$ und $\mathrm{M}$ an, aus irgendwelchen Gründen auch immer, und Jacksons (B) würde dahingehend umformuliert werden, sodass nun der Kern der schwachen Notwendigkeit erfasst werden würde, würde trotzdem ein Restproblem übrig bleiben. Jacksons (B) würde dann nicht zwischen schwacher Notwendigkeit (SN) und einer unendlich schwachen Möglichkeit (A1) differenzieren können. Das erste Problem besteht also darin, dass eine Reparatur von Jacksons $(B)$ in weiterer Folge wieder zu einem Differenzierungsproblem führt, nämlich zwischen (SN) und (A1).

Das zweite Problem besteht in der Willkür von (A1), wie viele Blocker nun möglich sind. Die Anzahl von Blockern reicht von eins bis unendlich. Wo soll ein Ende gesetzt werden? Die Akzeptanz eines Blockers öffnet die Türe für weitere Blocker und führt dadurch in die absurde Konsequenz einer unendlichen Reihe von fehlenden Blockern. Dieser Absurdität zu entgehen würde bedeuten, an einer Stelle willkürlich abzubrechen. Doch wenn wir in einer Reihe von fehlenden Blockern abbrechen wollen, warum fangen wir an erster Stelle überhaupt mit der Möglichkeit eines einzigen Blockers an? Genauso gut könnte man bereits vor der Einführung

\footnotetext{
${ }^{29}$ Natürlich ließe sich auch eine logisch schwächere Form darlegen, in der die Konjunktoren, welche die Blocker verbinden, durch Disjunktoren ersetzt werden.
} 
eines einzigen Blockers abbrechen und so eine schwache Notwendigkeit verhindern.

Hawthornes Blocker-Problem würde sich so gar nie ergeben. Der Vorzug wäre eine einfachere Darstellung der Relation zwischen $\mathrm{P}$ und $\mathrm{M}$.

\subsubsection{Fehlende und sich gegenseitig bedingende Blocker}

Falls die Möglichkeit von Blockern akzeptiert wird, dann erscheint auch die Möglichkeit von sich gegenseitig bedingenden Blockern gegeben zu sein, um (SN) noch weiter abzuschwächen. So macht erst die Summe sich gegenseitig bedingender fehlender Blocker in Kombination mit dem Physikalischen das Mentale notwendig. Das wechselseitige Bedingen ist derart, dass immer dann, wenn Blocker 1 abwesend ist, auch Blocker 2 abwesend ist, und wenn Blocker 2 abwesend ist, auch Blocker 3 abwesend ist, und so fort. Warum sollte die Summe der Blocker, deren Absenz notwendig für das Auftreten des Mentalen ist, nicht deutlich mehr als 10 sein oder mehr als 100 sein oder nicht gleich ins Unendliche gehen? Wenn nun mentale Entitäten (M) in Existenz treten, dann impliziert das Physikalische ( $P$, ausgedrückt in den Sätzen (1) und (2)) zusammen mit der Absenz von sich gegenseitig bedingenden Blockern ( $B 1$ bis $B \infty$ ) das Mentale. Diese Implikation ist nun wieder unendlich viel schwächer als (SN). Logisch sieht diese Art der Implikation in ihrer stärksten Form wie folgt aus ${ }^{30}$ :

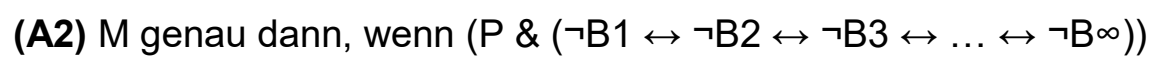

Hier ergeben sich wieder dieselben zwei Probleme wie bei (A1). Bei Gültigkeit einer schwachen Notwendigkeit zwischen $\mathrm{P}$ und $\mathrm{M}$ und einer angepassten Definition von Jacksons (B), würde auch diese Formulierung des Supervenienzphysikalismus nicht zwischen schwacher Notwendigkeit (SN) und einer unendlich schwachen Möglichkeit (A2) differenzieren können. Das erste Problem besteht also nochmal darin, dass eine Reparatur von Jacksons (B) in weiterer Folge wieder zu einem Differenzierungsproblem führt, nämlich zwischen (SN) und (A2).

Das zweite Problem besteht in der Willkür der Anzahl von Blockern in (A2), wie viele Blocker nun möglich sind. Die Akzeptanz eines Blockers öffnet die Türe für weitere sich wechselseitig bedingenden fehlenden Blockern und führt dadurch in die absurde Konsequenz einer unendlichen Reihe von sich wechselseitig bedingenden fehlenden Blockern. Dieser Absurdität zu entgehen würde bedeuten, an einer Stelle willkürlich abzubrechen. Mein Vorschlag ist auch hier, bereits die Einführung eines einzigen Blockers abzubrechen, sodass der einfacheren Relation zwischen $\mathrm{P}$ und M der Vorzug gegeben wird und Hawthornes BlockerProblem sich erst gar nicht ergibt.

${ }^{30}$ Auch hier ließe sich eine logisch schwächere Form darlegen, in der die Bisubjunktoren, welche die Blocker verbinden, durch Subjunktoren ersetzt werden. 


\subsubsection{Abwechselnd fehlende/anwesende Blocker}

Zu guter Letzt möchte ich eine letzte Möglichkeit aufzeigen, wie das Blocker-Problem beliebig weitergeführt werden kann. Falls die Möglichkeit von Blockern akzeptiert wird, dann erscheint auch die Möglichkeit von sich abwechselnd bedingenden fehlenden und vorhandenen Blockern gegeben zu sein, um (SN) noch weiter abzuschwächen. So macht erst die Summe sich abwechselnd bedingender fehlender und vorhandener Blocker in Kombination mit dem Physikalischen das Mentale notwendig. Das wechselseitige Bedingen ist derart, dass immer dann, wenn Blocker 1 abwesend ist, Blocker 2 anwesend sein muss, und wenn Blocker 3 abwesend ist, Blocker 4 anwesend sein muss, und so fort. Der Grund ist, dass Blocker 2 aktiv das Auftreten von Blocker 1 blockiert, wie auch immer das Blocker-zu-Blocker-Blockieren zu denken wäre, und Blocker 4 aktiv das Auftreten von Blocker 3 blockiert. Gleichzeitig tritt das erste Blocker-Paar (Blocker 1 und Blocker 2) genau dann auf, wenn auch das zweite BlockerPaar (Blocker 3 und Blocker 4) vorhanden ist, und so weiter. Warum sollte die Summe der fehlenden Blocker und der anwesenden Blocker nicht deutlich mehr als 10 sein oder mehr als 100 sein oder nicht gleich ins Unendliche gehen? Wenn nun mentale Entitäten (M) in Existenz treten, dann impliziert das Physikalische ( $P$, ausgedrückt in den Sätzen (1) und (2)) zusammen mit sich abwechselnd bedingenden fehlenden und vorhandenen Blockern ( $\mathrm{B} 1$ bis $\mathrm{B} \infty$ ) das Mentale. Diese Implikation ist nun wieder unendlich viel schwächer als (SN). Logisch sieht diese Art der Implikation in ihrer stärksten Form wie folgt aus ${ }^{31:}$

$$
\begin{aligned}
& \text { (A3) M genau dann, wenn }(\mathrm{P} \&(\neg \mathrm{B} 1 \leftrightarrow \mathrm{B} 2) \leftrightarrow(\neg \mathrm{B} 3 \leftrightarrow \mathrm{B} 4) \leftrightarrow \ldots \leftrightarrow(\neg \mathrm{B} \infty-1 \leftrightarrow \\
& \mathrm{B} \infty))
\end{aligned}
$$

Abseits davon, dass durch dieses Blockerszenario bereits ein ontologischer Dualismus eingeführt wird, weil das zweite Glied jedes Blockerpaares tatsächlich vorhanden sein muss, ergeben sich auch hier dieselben zwei Probleme wie bei (A1) und (A2). Bei Vorhandensein einer schwachen Notwendigkeit zwischen $\mathrm{P}$ und $\mathrm{M}$ und einer angepassten Definition von Jacksons (B), würde auch diese Formulierung des Supervenienzphysikalismus nicht zwischen schwacher Notwendigkeit (SN) und einer unendlich schwachen Möglichkeit (A3) differenzieren können. Das erste Problem besteht also wiederum darin, dass eine Reparatur von Jacksons (B) in weiterer Folge wieder zu einem Differenzierungsproblem führt, nämlich zwischen (SN) und (A3).

Das zweite Problem besteht in der willkürlichen Anzahl von abwesenden/anwesenden Blockern in (A3). Die Akzeptanz eines Blockers öffnet die Türe für weitere sich abwechselnd bedingenden fehlenden und vorhandenen Blocker und führt dadurch in die absurde Konsequenz einer unendlichen Reihe von sich wechselseitig bedingenden fehlenden und vorhandenen

\footnotetext{
${ }^{31}$ Auch in (A3) wäre eine logisch schwächere Form möglich, in der die Bisubjunktoren, welche die Blocker-Paare in den separierten Klammern sowie die Blocker-Paare untereinander verbinden, durch Subjunktoren ersetzt werden.
} 
Blockern. Dieser Absurdität zu entgehen, würde bedeuten, an einer Stelle willkürlich abzubrechen. Mein Vorschlag ist auch hier wieder derselbe wie bei den beiden anderen, nämlich bereits die Einführung eines einzigen Blockers abzubrechen, so dass der einfacheren Relation zwischen $\mathrm{P}$ und $\mathrm{M}$ der Vorzug gegeben wird und Hawthornes Blocker-Problem erst gar nicht vorgebracht werden kann.

Diese Art von Argumentation gegen das Blocker-Problem ließe sich noch weiterführen und es ließen sich noch komplexerer Blocker-Situationen entwickeln. Ich möchte es jedoch bei diesen drei vorgebrachten Problemen belassen und zum Abschluss noch ein drittes und letztes Problem für Anhänger von Hawthornes Blocker-Problem aufzeigen. Wenn nun die Möglichkeit eines Blockers zur Möglichkeit weiterer Blocker führt, so wie ich in (A1) bis (A3) argumentiert habe, so lässt sich im Falle der Gültigkeit einer reparierten Version von Jacksons (B) eigentlich nicht nur das Differenzierungsproblem zwischen (SN) und einem beliebigen Szenario (A1) bis (A3) für sich einzeln genommen vorbringen, sondern auch ein weiteres Differenzierungsproblem. Dieses weitere Differenzierungsproblem besteht nun darin, dass auch gar nicht zwischen den einzelnen Szenarien (A1) bis (A3) unterschieden werden kann. Somit ist das letzte Problem, dass (SN) nicht von (A1) oder (A2) oder (A3) oder jedem weiteren hinzukommenden Blocker-Szenario unterschieden werden kann.

\subsection{Zusammenfassung}

Ich habe in diesem Kapitel diskutiert, wie die Relation des vermeintlich Mentalen zum Physikalischen entwickelt werden kann. Dabei habe ich eine Übersicht zu den verschiedenen physikalistischen Positionen gegeben und immer wieder Querverbindungen zu den anderen Positionen hergestellt. Ich habe beim logischen Behaviorismus begonnen, der durch die Identitätstheorie abgelöst wurde. Sodann habe ich mich über den Eliminativismus und den Funktionalismus als Reaktion auf die Identitätsthese bis zum Repräsentationalismus und zur jüngsten Entwicklung des Fundierungsphysikalismus weitergehantelt. Abseits all ihrer Unterschiede haben alle physikalistischen Positionen die Supervenienz als gemeinsames Element. Die Supervenienzrelation besagt, dass es keine Änderung im Mentalen gibt, ohne auch eine Änderung im Physikalischen. Die Akzeptanz der Supervenienzrelation stellt die minimale Festlegung jedes Physikalisten dar, somit ist auch jegliche Version eines Physikalismus widerlegt, sobald die Supervenienz widerlegt ist. Genau hier kommt Hawthornes Blocker-Problem in Spiel, das sich gegen die Supervenienzrelation richtet. Die Kernaussage des Blocker-Problems ist, dass das Mentale nur deshalb auf dem Physikalischen superveniert, weil der Supervenienzrelation blockierende und gleichzeitig nicht-physikalische bzw. mentale Entitäten fehlen. Diese Möglichkeit kann der Supervenienzphysikalismus nicht ausschließen. Gegen dieses bisher ungelöste Problem für den Supervenienzphysikalismus richten sich meine 
drei eingeführten Blocker-Szenarien. Darin arbeite ich ein Differenzierungsproblem und ein Willkürproblem für alle blockerähnlichen Szenarien heraus, was zur Konklusion führt, Hawthornes Blocker gar nicht erst zuzulassen. Damit ist insgesamt in diesem Kapitel ein weiterer Schritt zu einer Formulierung des Physikalismus als eine substantielle These gelungen, indem gegen Hawthornes Blocker-Problem argumentiert wurde. 


\section{Dritter Exkurs E3: Eine Symmetrie-Relation zwischen möglichen Welten}

Die Supervenienzthese sagt, dass es keine Unterschiede in der mentalen Aufmachung ohne Unterschiede in der physikalischen Aufmachung gibt. Es ist unmöglich, dass irgendeine Veränderung der mentalen Aufmachung nicht auch durch eine physikalische Aufmachung hervorgebracht werden würde. Wie in Kapitel 3.2 dargelegt, ist Jacksons Version eines Supervenienzphysikalismus eines der einflussreichsten (Jackson, 1998a). Zur Erinnerung ist Jacksons Formulierung die folgende (S. 12):

(B) Jede Welt, die ein minimales physikalisches Duplikat unserer Welt ist, ist ein Duplikat unserer Welt schlechthin.

Eine mögliche Welt ist genau dann ein minimales physikalisches Duplikat unserer aktualen Welt, wenn es (a) exakt wie unsere aktuale Welt in jeder physikalischen Angelegenheit ist und (b) nichts anderes enthält, als es unbedingt enthalten muss, um (a) gerecht zu werden (S. 13). Jacksons Formulierung eines minimalen physikalischen Duplikates (M-Duplikat) als ein Duplikat schlechthin (S-Duplikat), erlaubt die Kompatibilität des Physikalismus mit der Möglichkeit von Geistern, cartesischen Seelen und vielen anderen fundamental mentalen Entitäten in irgendeiner möglichen Welt. Diese anderen möglichen Welten sind zwar physikalische Duplikate unserer aktualen Welt $w$, aber keine S-Duplikate, weil sie fundamental mentale Entitäten beherbergen, welche $w$ nicht beherbergt. Weil diese Welten keine S-Duplikate sind, sind sie auch keine M-Duplikate.

Somit ist der Physikalismus nicht kompatibel mit der Aktualität von fundamental mentalen Entitäten, aber er ist kompatibel mit deren Möglichkeit. Ebenso wird dadurch der Physikalismus kontingent, weil die Wahrheit des Physikalismus von den Gegebenheiten der aktualen Welt $w$, hinreichend ähnlichen Welten $w^{*} 1$ bis $w^{*} i$ als M-Duplikate und S-Duplikate sowie komplett unähnlichen Welten $w^{+} 1$ bis $w^{+} i$, die weder M-Duplikate noch S-Duplikate sind, abhängt.

Das Ziel dieses Exkurses ist es, zwei gegenwärtige Ansätze zu betrachten, die Jacksons (B) in einer formalisierten Art und Weise betrachten. Das bedeutet, dass ich die bereits vorgeschlagene partielle Ordnungsrelation zwischen möglichen Welten analysiere und zeige, warum beide Ansätze falsch sind. Daran anknüpfend entwickle ich meinen eigenen Ansatz einer Äquivalenzrelation zwischen möglichen Welten, die alle Probleme der partiellen Ordnungsrelation löst.

\section{E3.1. Eine partielle Ordnungsrelation zwischen Welten}

Der Ausgangspunkt ist die Frage, wie eine Relation zwischen möglichen Welten spezifischer beschrieben werden kann. Wenn alle möglichen Welten betrachtet werden, die auch gleichzeitig 
physikalische Duplikate sind, dann sind sie entweder M-Duplikate und S-Duplikate (d.h. $w^{*} 1$ bis $W^{*} i$ ) oder keine M-Duplikate und keine S-Duplikate (d.h. $w^{+} 1$ bis $\left.w^{+} i\right)$. Für diese möglichen Welten haben Leuenberger (2008, S. 159) und Chalmers (2012, S. 151-152) eine partielle Ordnungsrelation vorgeschlagen. Im Allgemeinen führen partielle Ordnungsrelationen Äquivalenzklassen ein, deren Elemente in allen essentiellen Aspekten vergleichbar sind. Um ein Beispiel zu bringen, führe ich die Äquivalenzklassen $E_{p}$ ein, die für alle physikalischen Duplikate von $w$ steht und ich führe dazu noch die möglichen Welten $w^{p} 1$ bis $w^{p} i$ ein, die für die einzelnen Elemente von $E_{p}$ stehen. An dieser Stelle sind wir nicht a priori darauf verpflichtet, dass alle Elemente von $E_{p}$ auch M-Duplikate und S-Duplikate sind, denn diese Frage hat die partielle Ordnungsrelation zu klären. Somit verwende ich $w^{p} i$ als ein physikalisches Duplikat, dass schließlich entweder zu $w^{*} i$ und damit zu einem M-Duplikat und S-Duplikat wird, oder $z u w^{+} i$ und somit zu keinem M-Duplikat und keinem S-Duplikat wird. Der Ausdruck " $w^{0} i$ " ist damit lediglich ein Platzhalter, weil die zu prüfende mögliche Welt schlussendlich in $w^{*} i$ oder $w^{+} i$ kollabiert. Dieser Unterschied wird im Zuge meines Arguments an späterer Stelle wichtig. Darüber hinaus ist die Relation zwischen den Elementen von $E_{p}$ reflexiv (d.h. $w^{p} 1=w^{p} 1$ ), transitiv (d.h. wenn $w^{p} 1$ $\leq w^{p} 2$ und $w^{p} 2 \leq w^{p} 3$, dann $w^{p} 1 \leq w^{p} 3$ ) und antisymmetrisch (d.h. wenn $w^{p} 1 \leq w^{p} 2$ und $w^{p} 2 \leq w^{p} 1$, dann $\left.w^{p} 1=w^{p} 2\right)$. Bei Leuenberger ist eine mögliche Welt $w^{p} 1$ in $E_{p}$ genau dann ein M-Duplikat, wenn irgendeine andere mögliche Welt $w^{p} i$ nicht $z u w^{p} 1$ identisch ist, sodass $w^{p} i$ nicht kleiner gleich $w^{p} 1$ ist. Ich verstehe den Ausdruck "kleiner gleich" zwischen Welten derart, dass damit die mentale Aufmachung der involvierten Welten verglichen wird. Anders formuliert ist $w^{p} 1$ in $E_{p}$ genau dann ein M-Duplikat, wenn $w^{p} i$ und $w^{p} 1$ verschiedene Welten sind und $w^{p} i$ keine geringere mentale Aufmachung als $w^{p} 1$ hat oder dieselbe mentale Aufmachung als $w^{p} 1$ hat. Als Konsequenz gibt es Geister oder cartesische Seelen in $w^{p} i$, aber nicht in $w^{p} 1$. Somit ist $w^{p} 1$ ein M-Duplikat und ein S-Duplikat der aktualen Welt $w^{32}$ :

(L1) $w^{p} 1$ ist ein M-Duplikat in $E_{p}$, gdw. es nicht der Fall ist, dass $w^{p} i \leq w^{p} 1$.

Oder in einer alternativen Formulierung durch die Negation von "kleiner gleich":

(L2) $w^{p} 1$ ist ein M-Duplikat in $E_{p}$, gdw. $w^{p} 1<w^{p} i$.

Wenden wir uns nun der zweiten Explikation einer partiellen Ordnungsrelation bei Chalmers zu. Chalmers bringt die ähnliche Idee in einem etwas anderen Vokabular vor ${ }^{33}$. In seinem Ansatz ist eine mögliche Welt $w^{p} 1$ in $E_{p}$ genau dann ein M-Duplikat, wenn $w^{p} 1$ irgendeine andere Welt $w^{p} i$ nicht übersteigt (to outstrip). Ich verstehe unter "übersteigen" in Bezug auf Welten, dass damit "größer als" gemeint ist hinsichtlich der mentalen Aufmachung. Beispielsweise übersteigt $w^{p} 1$

\footnotetext{
32 Ich verwendet nachfolgend den Ausdruck "gdw." als Abkürzung für "genau dann, wenn".

33 Jackson selbst bestätigt diese Formulierung in Chalmers und Jackson (2001, S. 317). 
eine andere mögliche Welt $w^{p} i$ genau dann, wenn $w^{p} 1$ Geister oder cartesische Seelen beinhaltet, die $w^{p} i$ nicht beinhaltet. Also wenn $w^{p} 1$ in $E_{p}$ keine andere mögliche Welt $w^{p} i$ übersteigt, dann gibt es auch keine Geister oder cartesische Seelen in $w^{p} 1$, jedoch gibt es sie in $w^{p} i$. Somit ist $w^{p} 1$ ein M-Duplikat und ein S-Duplikat der aktualen Welt $w$ :

(C1) $w^{p} 1$ ist ein M-Duplikat in $E_{p}$, gdw. es nicht der Fall ist, dass $w^{p} 1>w^{p} i$.

Oder in einer alternativen Formulierung durch die Negation von "größer als":

(C2) $w^{p} 1$ ist ein M-Duplikat in $E_{p}$, gdw. $w^{p} 1 \leq w^{p}$.

Nehmen wir an, dass $w^{p} 1$ eine Welt ist, die auch gleichzeitig ein M-Duplikat und S-Duplikat von $w$ ist. Führen wir auch $w^{p} 2$ als eine Welt mit Ektoplasma und cartesischen Seelen ein sowie $w^{p} 3$ als eine Welt mit Ektoplasma, cartesischen Seelen und Geistern. Die mögliche Welt $w^{p} 1$ ist identisch zu sich selbst, somit ist die Reflexivitätsrelation erfüllt, sodass $w^{p} 1=w^{p} 1$ gilt. Ebenso enthält $w^{p} 1$ eine geringere mentale Aufmachung (d.h. keine Mentalität) als $w^{p} 2$, die wiederum eine geringere mentale Aufmachung als $w^{p} 3$ enthält. Daraus folgt, dass $w^{p} 1$ eine geringere mentale Aufmachung als $w^{p} 3$ enthält. Dieser Sachverhalt bedeutet, dass auch die Transitivitätsrelation erfüllt ist (d.h. wenn $w^{p} 1 \leq w^{p} 2$ und $w^{p} 2 \leq w^{p} 3$, dann $w^{p} 1 \leq w^{p} 3$ ). Überraschenderweise ist jedoch die Antisymmetrie verletzt, weil $w^{p} 2$ mehr Mentalität beinhaltet als $w^{p} 1$, weshalb $w^{p} 2 \leq w^{p} 1$ nicht gegeben ist und damit auch $w^{p} 1=w^{p} 2$ nicht gegeben ist. Wenn jedoch (L2) angewendet wird, dann wird aus $w^{p} 1$ ein M-Duplikat, weil es andere Welten gibt, die eine größere mentale Aufmachung als $w^{p} 1$ haben. Ähnliches ergibt sich, wenn (C2) angewendet wird. Auch hier wird aus $w^{p} 1$ ein M-Duplikat, weil es andere Welten gibt, die eine größere mentale Aufmachung als $w^{p} 1$ haben oder dieselbe mentale Aufmachung wie $w^{p} 1$ haben.

Das Problem mit dieser Situation ist, dass die Antisymmetrierelation, die ein definitorischer Teil einer partiellen Ordnungsrelation ist, verletzt ist. Es gibt keine partielle Ordnungsrelation, wenn die Welten in $E_{p}$ nicht antisymmetrisch sind. Also ist eine partielle Ordnungsrelation zwischen Welten, so wie sie von Leuenberger und Chalmers vorgeschlagen werden, zur adäquaten Klassifikation von M-Duplikaten nicht geeignet, weil einer der folgenden beiden Fälle zutrifft. Entweder ist $w^{p} 1$ ex hypothesi ein M-Duplikat unter Anwendung von (L2) und (C2), das jedoch so nicht sein sollte, weil die Antisymmetrierelation verletzt ist, oder $w^{p} 1$ ist kein M-Duplikat aufgrund des Fehlens einer Antisymmetrie, was jedoch von (L2) und (C2) als solches nicht richtig identifiziert wurde ${ }^{34}$.

\footnotetext{
${ }^{34}$ Neben diesen Gemeinsamkeiten on (L2) und (C2) sollte auch ein offensichtlicher Unterschied benannt werden. Bei Leuenbergers (L2) muss ein M-Duplikat die Bedingung erfüllen, kleiner als irgendeine andere mögliche Welt zu sein, wohingegen bei Chalmers' (C2) ein M-Duplikat die Bedingung erfüllen muss, kleiner oder gleich als irgendeine andere mögliche Welt zu sein. Für eine andere Explikation von Leuenbergers Ansatz siehe Blumson und Tang (2015).
} 
In Kontrast zu unserem ersten Beispiel führe ich nun $w^{p} 1$ als eine Welt ein, in der es Ektoplasma gibt. Die Reflexivitätsrelation ist gegeben, weil $w^{p} 1$ identisch zu sich selbst ist, sodass $w^{p} 1=w^{p} 1$ gilt. Die Transitivitätsrelation ist ebenfalls gegeben, denn falls $w^{p} 1$ Ektoplasma beinhaltet, $w^{p} 2$ Ektoplasma und cartesische Seelen beinhaltet und $w^{p} 3$ Ektoplasma, cartesische Seelen und Geister beinhaltet, dann gilt $w^{p} 1 \leq w^{p} 2, w^{p} 2 \leq w^{p} 3$ und somit ist auch $w^{p} 1 \leq w^{p} 3$ korrekt. Die Antisymmetrie ist jedoch wieder verletzt. Der Grund dafür ist, dass $w^{p} 2 \mathrm{mehr}$ Mentalität beinhaltet als $w^{p} 1$, somit gilt $w^{p} 2 \leq w^{p} 1$ nicht und $w^{p} 1=w^{p} 2$ ist auch nicht gültig. Überraschenderweise ist bei Anwendung von (L2) $w^{p} 1$ ein M-Duplikat, weil es Welten mit einer größeren mentalen Aufmachung als $w^{p} 1$ gibt. Doch $w^{p} 1$ sollte kein M-Duplikat sein, da es fundamental Mentales in Form eines Ektoplasmas beinhaltet. Ein ähnliches Ergebnis liefert die Anwendung von (C2), das $w^{p} 1$ zu einem M-Duplikat macht, weil es Welten mit einer größeren mentalen Aufmachung als $w^{p} 1$ gibt oder Welten mit derselben mentalen Aufmachung wie $w^{p} 1$ gibt. Aber auch hier sollte $w^{p} 1$ kein M-Duplikat sein, da es Ektoplasma enthält.

Somit ist eine partielle Ordnungsrelation zwischen Welten, so wie sie von Leuenberger und Chalmers vorgeschlagen werden, zur adäquaten Klassifikation von physikalischen Duplikaten, die keine M-Duplikate und keine S-Duplikate sind, nicht geeignet, weil es wieder zu folgendem Dilemma führt. Entweder ist $w^{p} 1$ ex hypothesi kein M-Duplikat aufgrund des Fehlens einer Antisymmetrie, doch es wird unter Anwendung von (L2) und (C2) fälschlicherweise als ein M-Duplikat klassifiziert, oder $w^{p} 1$ ist ein M-Duplikat aufgrund von (L2) und (C2), doch es sollte kein M-Duplikat sein. Letzteres wird jedoch von (L2) und (C2) nicht richtig erfasst.

\section{E3.2. Die Einführung von zwei Äquivalenzklassen}

Um einige dieser Probleme zu lösen, schlage ich vor, die Unbestimmtheit des Begriffes "physikalisches Duplikat" aufzulösen. Im Kontext der Diskussion über physikalische Duplikate, und ob sich diese nun als M-Duplikate qualifizieren oder auch nicht, ist es ein Kategorienfehler, alle physikalischen Duplikate in eine einzige Äquivalenzklasse zu geben, unabhängig von deren Status als M-Duplikate oder eben dem nicht vorhandenen Status. Die Unbestimmtheit von "physikalisches Duplikat" verhindert demnach, mögliche Welten, die auch gleichzeitig MDuplikate sind, in die richtige Äquivalenzklasse zu geben. Eine richtige Zuordnung ist jedoch durch eine Einführung von zwei Äquivalenzklassen möglich, eine für M-Duplikate und eine für physikalische Duplikate, die keine M-Duplikate sind. Zur Erinnerung: Das Ziel einer Äquivalenzklasse ist eine hinreichende Ähnlichkeit der wesentlichen Merkmale der darin enthaltenen Elemente.

Bis jetzt wurde lediglich die Äquivalenzklasse $E_{p}$ eingeführt, die für alle physikalischen Duplikate von $w$ stehen und deren Elemente von $w^{p} 1$ bis $w^{p} i$ reichen. Basierend auf dieser Einführung und den Explikationen von Leuenbergers und Chalmers' Ansätzen zur relevanten 
partiellen Ordnungsrelation, sind wir an einigen unliebsamen Problemen angelangt. Nehmen wir den Unterschied zwischen M-Duplikaten und simplen physikalischen Duplikaten ernst. Sohin führe ich als erstes die Äquivalenzklasse $E_{p}{ }^{*}$ ein, deren Elemente alle M-Duplikate und SDuplikate von $w$ sind, und von $w^{*} 1$ bin $w^{*} i$ reichen. Als zweites führe ich die Äquivalenzklasse $E_{p}{ }^{+}$ein, deren Elemente alle physikalischen Duplikate sind, die keine M-Duplikate und keine SDuplikate sind, und von $w^{+} 1$ bin $w^{+} i$ reichen. Das führt zu den folgenden Revisionen von (L2) und (C2) hin zu bereichsspezifischen Thesen:

(L2*) $w^{*} 1$ ist ein M-Duplikat in $E_{p}{ }^{*}$, gdw. $w^{*} 1<w^{*} i$

$\left(\mathbf{L 2}^{+}\right) w^{+} 1$ ist ein M-Duplikat in $E_{p}^{+}$, gdw. $w^{+} 1<w^{+} i$

$\left(\mathbf{C} 2^{*}\right) w^{*} 1$ ist ein M-Duplikat in $E_{p}{ }^{*}, \mathrm{gdw} . w^{*} 1 \leq w^{*} i$

$\left(\mathrm{C2}^{+}\right) w^{+} 1$ ist ein M-Duplikat in $E_{p}^{+}, \mathrm{gdw} . w^{+} 1 \leq w^{+} i$

Legen wir fest, dass das Triplet von M-Duplikaten ( $w^{*} 1$ bis $w^{*} 3$ ) keine fundamental mentalen Entitäten enthält. Unter der Voraussetzung einer partiellen Ordnungsrelation erfüllt die mögliche Welt $w^{*} 1$ als ein M-Duplikat in $E_{p}{ }^{*}$ alle notwendigen relationalen Eigenschaften. Das bedeutet, dass $w^{*} 1$ identisch mit sich selbst ist, sodass die Reflexivitätsrelation und gleichsam $w^{*} 1=w^{*} 1$ erfüllt sind. Ebenfalls ist die Transitivitätsrelation gegeben, denn falls $w^{*} 1$ verglichen mit $w^{*} 2$ eine geringere mentale Aufmachung oder dieselbe mentale Aufmachung (d.h. keine Mentalität) beinhaltet und $w^{*} 2$ verglichen mit $w^{*} 3$ eine geringere mentale Aufmachung oder dieselbe mentale Aufmachung (d.h. nochmals keine Mentalität) beinhaltet, dann gilt $w^{*} 1 \leq w^{*} 2, w^{2} \leq w^{*} 3$ und somit ist auch $w^{*} 1 \leq w^{*} 3$ korrekt. Zu guter Letzt ist auch die Antisymmetrierelation erfüllt, denn $w^{*} 1$ verglichen mit $w^{*} 2$ enthält eine geringere oder dieselbe mentale Aufmachung. Da es in beiden Welten keine Mentalität gibt, gibt es auch keine unterschiedliche Anzahl an Entitäten, weshalb auch $w^{*} 2$ verglichen mit $w^{*} 1$ eine geringere oder dieselbe mentale Aufmachung enthält. Als Konklusion ist die Relation $w^{*} 1=w^{*} 2$ erfüllt. Wenn $\left(\mathrm{L}^{*}\right)$ angewendet wird, wird die mögliche Welt $w^{*} 1$ in $E_{p}{ }^{*}$ nicht als ein M-Duplikat klassifiziert, weil $w^{*} 1$ keine geringere mentale Aufmachung als $w^{*} 2$ aufweist. Leuenbergers Formulierung kommt also zu einem falschen Ergebnis. Wird hingegen $\left(\mathrm{C}^{*}\right)$ angewendet, wird die mögliche Welt $w^{*} 1$ in $E_{p}{ }^{*}$ als ein M-Duplikat klassifiziert, weil $w^{*} 1$ eine geringere mentale Aufmachung als $w^{*} 2$ oder dieselbe mentale Aufmachung wie $w^{*} 2$ aufweist. Damit kommt nur Chalmers' Formulierung zum richtigen Ergebnis.

Betrachten wir nun ein Triplet von simplen physikalischen Duplikaten, die alle etwas fundamental Mentales beinhalten. Die mögliche Welt $w^{+} 1$ beinhaltet Ektoplasma, $w^{+} 2$ beinhaltet Ektoplasma und cartesische Seelen und $w^{+} 3$ beinhaltet Ektoplasma, cartesische Seelen und Geister. Die mögliche Welt $w^{+} 1$ in $E_{p}{ }^{+}$ist reflexiv, weil sie mit sich selbst identisch ist und dadurch $w^{+} 1=w^{+} 1$ gilt. Die Transitivitätsrelation ist ebenso gegeben, denn falls $w^{+} 1$ eine 
geringere mentale Aufmachung als $w^{+} 2$ oder dieselbe mentale Aufmachung wie $w^{+} 2$ beinhaltet und $w^{+} 2$ wiederum eine geringere mentale Aufmachung als $w^{+} 3$ oder dieselbe mentale Aufmachung wie $w^{+} 3$ beinhaltet, dann gilt $w^{+} 1 \leq w^{+} 2, w 2 \leq w^{+} 3$ und somit ist auch $w^{+} 1 \leq w^{+} 3$ korrekt. Schließlich ist die Antisymmetrierelation verletzt, weil $w^{+} 1$ weniger Mentalität als $w^{+} 2$ oder dieselbe Mentalität wie $w^{+} 2$ enthält, jedoch $w^{+} 2$ mehr Mentalität als $w^{+} 1$ enthält. Somit ist $w^{+} 2 \leq w^{+} 1$ nicht gültig und daraus folgend auch $w^{+} 1=w^{+} 2$ nicht korrekt. Bei Anwendung von $\left(\mathrm{L}^{+}\right)$und $\left(\mathrm{C} 2^{+}\right)$wird überraschenderweise $w^{+} 1$ als ein M-Duplikat klassifiziert, obwohl es ex hypothesi ein simples physikalisches Duplikat ist, weil $w^{+} 1$ eine geringere mentale Aufmachung als $w^{+} 2$ oder dieselbe mentale Aufmachung wie $w^{+} 2$ enthält. Dieses Ergebnis ist problematisch, weil $w^{+} 1$ bei einer adäquaten Formulierung eines minimalen physikalischen Duplikates nicht als M-Duplikat klassifiziert werden sollte, insbesondere auch deshalb, weil die Antisymmetrierelation von vornherein verletzt ist. Somit kommen sowohl Leuenbergers als auch Chalmers' Formulierungen zum falschen Ergebnis.

Zusammengefasst führen die analysierten partiellen Ordnungsrelationen zwischen möglichen Welten in der Äquivalenzklasse $E_{p}{ }^{*}$ der M-Duplikate und der Äquivalenzklasse $E_{p}{ }^{+}$ der simplen physikalischen Duplikate nicht in jedem Fall zu einem richtigen Ergebnis. Einerseits klassifiziert Leuenbergers Version (L2*) ein M-Duplikat und damit S-Duplikat nicht als solches, sondern nur Chalmers' Version (C2*) führt zu einer korrekten Klassifikation von M-Duplikaten und damit S-Duplikaten. Andererseits liefern beide inkorrekte Ergebnisse in der Klassifizierung von simplen physikalischen Duplikaten, die keine M-Duplikate und keine S-Duplikate sind. Das Problem mit Leuenbergers $\left(\mathrm{L} 2^{+}\right)$und Chalmers' $\left(\mathrm{C} 2^{+}\right)$ist, dass $w^{+} 1$ als ein M-Duplikat ausgegeben wird, aber es sollte kein M-Duplikat sein.

\section{E3.3. Die Annahme einer Äquivalenzrelation zwischen Welten}

Was wir in den vorigen Abschnitten gelernt haben, ist die Einsicht, dass Jacksons (B) nicht zufriedenstellend in eine partielle Ordnungsrelation übersetzt werden kann. Die intuitive Idee hinter (B), so wie ich es verstehe, ist die Kernaussage, dass eine Kopie der gesamten physikalischen Aufmachung in w, ohne irgendetwas Weiteres hinzuzufügen, eine Kopie der gesamten mentalen Aufmachung nach sich zieht. Jede mögliche Welt als ein M-Duplikat und somit S-Duplikat ist dadurch eine perfekte Kopie von w. Das ist gleichzusetzen mit der Aussage, dass ein M-Duplikat auch denselben physikalischen und mentalen Inhalt wie $w$ hat und vice versa. Zusätzlich bedeutet der Umstand, eine perfekte Kopie zu sein, dass auch alles andere kopiert wird wie beispielsweise Gesetzmäßigkeiten zwischen der (mikro- und makro)physikalischen Aufmachung. Einfach alles, das einem in den Sinn kommt: Wenn es in $w$ ist, dann ist es auch ein Teil einer perfekten Kopie. Falls das zutrifft, dann ist die Relation zwischen einem M-Duplikat und $w$ nicht nur antisymmetrisch, sondern symmetrisch. Dadurch wird eine 
Äquivalenzrelation zwischen möglichen Welten den Intuitionen hinter (B) gerechter als eine partielle Ordnungsrelation. Um nun auch die anderen Probleme des vorigen Abschnittes zu lösen, führe ich eine Äquivalenzrelation zwischen möglichen Welten in der Äquivalenzklasse $E_{p}{ }^{*}$, deren Elemente M-Duplikate sind, und in der Äquivalenzklasse $E_{p}{ }^{+}$, deren Elemente simple physikalische Duplikate und keine M-Duplikate sind, ein.

Eine Äquivalenzrelation zwischen den Elementen von $E_{p}{ }^{*}$ ist reflexiv, transitiv und symmetrisch (d.h. $w^{*} 1=w^{*} 2$ und $w^{*} 2=w^{*} 1$ ). $A$ fortiori ist $w^{*} 1$ in $E_{p}{ }^{*}$ ein M-Duplikat genau dann, wenn $w^{*} 1$ genau dieselbe mentale Aufmachung hat wie $w^{*} i$. Nicht mehr und nicht weniger. Es gibt keinen Platz verschiedener Interpretationen, so wie es bei Leuenbergers und Chalmers' verschiedenen und nicht-äquivalenten Explikationen der partiellen Ordnungsrelation der Fall war. Die Äquivalenzrelation zwischen möglichen Welten sichert die folgende Formulierung:

(B1*) $w^{*} 1$ ist ein M-Duplikat in $E_{p}{ }^{*}, \mathrm{gdw} . w^{*} 1=w^{*}$.

Legen wir hier fest, dass das Triplet von M-Duplikaten ( $w^{*} 1$ bis $w^{*} 3$ ) keine fundamental mentalen Entitäten enthält. Bei Verwendung einer Äquivalenzrelation erfüllt die mögliche Welt $w^{*} 1$ als M-Duplikat in $E_{p}{ }^{*}$ alle dafür notwendigen relationalen Eigenschaften. Es ist die Reflexivität erfüllt, denn $w^{*} 1$ ist identisch mit sich selbst und deshalb ist $w^{*} 1=w^{*} 1$ erfüllt. Es ist auch die Transitivitätsrelation erfüllt, denn wenn $w^{*} 1$ verglichen mit $w^{*} 2$ enthält eine geringere mentale Aufmachung oder dieselbe mentale Aufmachung (d.h. keine Mentalität) und $w^{*} 2$ verglichen mit $w^{*} 3$ enthält eine geringere mentale Aufmachung oder dieselbe mentale Aufmachung (d.h. wieder keine Mentalität), dann gilt auch $w^{*} 1 \leq w^{*} 2, w 2 \leq w^{*} 3$ und somit ist auch $w^{*} 1 \leq w^{*} 3$ erfüllt. Schließlich ist auch die Symmetrierelation erfüllt. Denn $w^{*} 1$ verglichen mit $w^{*} 2$ enthält dieselbe mentale Aufmachung (d.h. keine Mentalität) und vice versa, somit ist auch $w^{*} 1=w^{*} 2$ gültig. Gemäß $\left(\mathrm{B} 1^{*}\right)$ ist $w^{*} 1$ auch ein M-Duplikat in $E_{p}{ }^{*}$, weil $w^{*} 1$ und $w^{*} 2$ dieselbe mentale Aufmachung beinhalten. Deshalb liefert (B1*) die richtigen Ergebnisse.

In derselben Argumentationslinie bedeutet eine Äquivalenzrelation zwischen den Elementen von $E_{p}{ }^{+}$, dass diese reflexiv, transitiv und symmetrisch (d.h. $w^{*} 1=w^{*} 2$ und $w^{*} 2=$ $\left.W^{*} 1\right)$ ist. $A$ fortiori ist $w^{+} 1$ ein M-Duplikat in $E_{p}^{+}$genau dann, wenn $w^{+} 1$ dieselbe mentale Aufmachung wie $w^{+} 2$ beinhaltet. Die Äquivalenzrelation zwischen möglichen Welten sichert die folgende Formulierung:

$\left(\mathbf{B 1}^{+}\right) w^{+} 1$ ist ein M-Duplikat in $E_{p}^{+}, \mathrm{gdw} . w^{+} 1=w^{+} i$.

Betrachten wir nun ein Triplet von simplen physikalischen Duplikaten, die alle etwas fundamental Mentales beinhalten. Ich möchte festlegen, dass $w^{+} 1$ eine mögliche Welt mit Ektoplasma, $w^{+} 2$ eine mögliche Welt mit Ektoplasma und cartesischen Seelen und $w^{+} 3$ eine mögliche Welt mit Ektoplasma, cartesischen Seelen und Geistern darstellt. Die mögliche Welt 
$w^{+} 1$ in $E_{p}^{+}$ist reflexiv, weil $w^{+} 1$ ist identisch mit sich selbst und so ist auch $w^{+} 1=w^{+} 1$ gültig. Des Weiteren ist auch die Transitivitätsrelation gegeben, weil $w^{+} 1$ weniger Mentalität beinhaltet als $w^{+} 2$ und $w^{+} 2$ wiederum weniger Mentalität beinhaltet als $w^{+} 3$, weshalb $w^{+} 1 \leq w^{+} 2, w^{+} 2 \leq w^{+} 3$ und damit auch $w^{+} 1 \leq w^{+} 3$ gültig ist. Doch falls $w^{+} 1$ mit $w^{+} 2$ verglichen wird, dann beinhalten beide Welten nicht dieselbe mentale Aufmachung, sodass $w^{+} 1=w^{+} 2$ und $w^{+} 2=w^{+} 1$ nicht korrekt ist. Somit ist die Symmetrierelation verletzt. Gemäß $\left(B 1^{+}\right)$ist $w^{+} 1$ auch kein M-Duplikat in $E_{p}^{+}$, weil $w^{*} 1$ and $w^{*} 2$ eine verschiedene mentale Aufmachung beinhalten. Deshalb liefert $\left(\mathrm{B} 1^{+}\right)$die richtigen Ergebnisse.

Wir sind nun bei einer formalisierteren Version von Jacksons (B) angelangt. Eine Äquivalenzrelation zwischen möglichen Welten klassifiziert nicht nur M-Duplikate und dadurch S-Duplikate als solche in $E_{p}{ }^{*}$, sondern auch simple physikalische Duplikate als solche in $E_{p}{ }^{+}$, die keine M-Duplikate und keine S-Duplikate sind.

\section{E3.4. Konklusion}

Zusammenfassend lässt sich festhalten, dass eine formalere Beschreibung von Jacksons Supervenienzphysikalismus durch eine partielle Ordnungsrelation zwischen möglichen Welten verschiedene Probleme aufwirft. Erstens, wenn alle physikalischen Duplikate unserer aktualen Welt betrachtet werden, dann identifizieren beide Ansätze von Leuenberger und Chalmers weder M-Duplikate noch simple physikalische Duplikate als solches. Zweitens, wenn die jeweilige Äquivalenzklasse der M-Duplikate und simplen physikalischen Duplikate separat betrachtet werden, dann identifiziert nur Chalmers' Ansatz, aber nicht Leuenbergers Ansatz, M-Duplikate als solche. Zudem versagen beide Ansätze bei der Identifikation von simplen physikalischen Duplikaten als solche. Als Konsequenz ist eine partielle Ordnungsrelation nicht zur näheren Beschreibung von Jacksons Supervenienzphysikalismus geeignet, weil es bei einigen Anwendungen falsche Resultate hervorbringt.

Eine natürliche Re-Interpretation von Jacksons Passage führt zu einer Symmetrierelation zwischen M-Duplikaten, die weiterfolgend unter einer Äquivalenzrelation zwischen möglichen Welten Verwendung findet. Bei dieser Äquivalenzrelation werden sowohl M-Duplikate als auch simple physikalische Duplikate als solche identifiziert. Selbstverständlich hängt meine gesamte Argumentation davon ab, welche Elemente zu einer bestimmten Äquivalenzrelation gezählt werden, wie ich Jacksons Supervenienzphysikalismus auffasse und wie ich die bisher vorhandenen partiellen Ordnungsrelationen interpretiere. Die von mir aufgeworfenen Probleme einer partiellen Ordnungsrelation und meine Lösungen dazu zeigen zumindest die Notwendigkeit, der exakten Relation zwischen möglichen Welten größerer Beachtung zu schenken, als sie es bisher erfahren hat. Denn davon hängt ab, wie wir modale Definitionen des Physikalismus bewerten können. 


\section{We'll rise again \\ Just like the phoenix \\ We'll rise again}

Tenacious D (2012) 


\section{Der neuerliche Aufstieg der Identitätstheorie}

In diesem Kapitel möchte ich als erstes Ziel Entscheidungskriterien aufstellen, die systematisch den Vergleich mehrerer konkurrierender Theorien ermöglichen und schließlich in der Argumentation für die Identitätstheorie maßgeblich sind. Es handelt sich um die beiden Kriterien der Einfachheit und Sparsamkeit, die bereits in der Physikalismusdebatte Anwendung gefunden haben (z.B. Smart, 1959). Das zweite Ziel betrifft zu zeigen, dass die Supervenienzrelation keine hinreichende Erklärung für den Zusammenhang zwischen dem Physikalischen und dem Mentalen ist, sehr wohl aber eine Identitätsrelation. Eine Identität zwischen dem Physikalischen und dem Mentalen erklärt deren gemeinsames Auftreten am besten. Als Haupteinwand gegen die Identitätstheorie wird das Argument der multiplen Realisierbarkeit gebracht und es scheint kein Weg daran vorbei zu gehen. Übereinstimmend mit der neueren Literatur zur multiplen Realisierbarkeit argumentiere ich als drittes Ziel, dass bei näherer Betrachtung dieses Argument verschwindet und nicht mehr als Todesstoß für die Identitätstheorie ins Treffen geführt werden kann. Als viertes Ziel spezifiziere ich die Identitätstheorie näher als eine A-priori-Theorie und argumentiere gegen Anhänger einer A-posteriori-Spielart der Identitätstheorie.

\subsection{Kriterien wissenschaftlicher Theorien}

Ob nun ein bestimmtes Paradigma, bestehend aus einer Theorie oder mehrerer Theorien, durch ein anderes Paradigma aufgrund ihrer Inkommensurabilität komplett ersetzt wird (Kuhn, 1967), oder ein bestimmtes Forschungsprogramm, bestehend aus Theorien und Methoden, ständig weiterentwickelt wird (Lakatos, 1982), oder eine Theorie durch Falsifikation komplett aufzugeben ist, weil zumindest eine ihrer Prämissen widerlegt wurde (Popper, 1934), oder wissenschaftliche Theorien immer nur in Kombinationen mit weiteren zusätzlichen Hintergrundannahmen zu sehen sind (Duhem, 1998; Quine, 1951), ist hier nicht die Kernfrage. Die wesentliche Frage ist, welche Kriterien eine wissenschaftliche Theorie erfüllen muss, um überhaupt als eine annehmbare Theorie zu gelten, und welcher Theorie bei einem Vergleich mit Konkurrenztheorien der Vorzug zu geben ist. Hierzu gibt es wieder viele Standpunkte, abhängig von den dahinterliegenden wissenschaftstheoretischen Überzeugungen und mehr oder weniger abhängig von der Nuancierung der zugeschriebenen Wichtigkeit.

Beispielsweise ist im logischen Positivismus die Verifizierbarkeit das wesentlichste Kriterium einer Theorie, im kritischen Rationalismus ist es die Falsifizierbarkeit. In beiden Richtungen kommen jedoch auch weiteren Kriterien, wie z.B. der Widerspruchsfreiheit der zugrunde gelegten Theorien und deren Vorhersagekraft, Bedeutung zu. Wieder andere bevorzugen konservative Theorien, welche die bisher bestehenden Theorien nur geringfügig ändern (z.B. Quine \& Ullian, 1970), wobei andere schneller Theorien akzeptieren, die komplett verschieden zu den bisherigen Theorien sind (z.B. Anhänger von Lakatos' Ansatz). Ich möchte 
keinen systematischen Vergleich der Kriterien guter wissenschaftlicher Theorien zwischen verschiedenen Autoren vorlegen, sondern werde auf zwei Merkmale eingehen, die in der Philosophie des Geistes und der Physikalismusdebatte immer wieder auftauchen. Es handelt sich um die beiden Merkmale der Einfachheit und der Sparsamkeit, die im direkten Vergleich zweier Theorien (T1 vs. T2) den Ausschlag in die eine oder eben in die andere Richtung geben: Die einfachere und sparsamere Theorie gewinnt das Rennen.

Was nun genau unter "Einfachheit" zu verstehen ist, möchte ich hier exemplarisch einführen. Ein Abriss in die Literatur zeigt, dass "Einfachheit" nicht eindeutig und übereinstimmend verwendet wird, was vor allem Bunge (1963) als Kritikpunkt gegen dieses Merkmal wissenschaftlicher Theorien vorbringt. So wird damit unter anderem eine Reduktion ontologischer Verpflichtung verstanden (Huemer, 2009), eine unkomplizierte und wenig komplexe Relation zwischen gegebenen Sachverhalten, wie beispielsweise lineare Zusammenhänge von empirischen Datenpunkten im Vergleich zu nicht-linearen Zusammenhängen (Sober, 1998), oder es wird damit eine geringe Anzahl und geringe Komplexität von Gesetzen, Grundannahmen und Postulaten gemeint (Brenner, 2017). Weiters verstehen andere wiederum unter einer ontologischen Sparsamkeit eine "semantische Einfachheit" (Sober, 2001) und unter einer Sparsamkeit an Gesetzen, Grundannahmen und Postulaten eine "syntaktische Einfachheit" (Baker, 2011). Eine weitere Übersicht über die gegenwärtige Literatur würde noch mehr Unterscheidungen und unterschiedliche Nuancen der Bedeutung einer Einfachheit bringen. Eine Einsicht möchte ich jedoch betonen, nämlich die, dass das Kriterium der Einfachheit teilweise über eine ontologische Sparsamkeit definiert wird. Ich bin mir nicht sicher, ob das ein guter Weg eines Verständnisses der Einfachheit ist. Ich bin mir jedoch sicher, dass beide Merkmale wichtig sind, gleichsam werden beide Merkmale in meiner nachfolgenden Argumentation separat eine Rolle spielen. Aus diesem Grunde möchte ich für den Rest dieser Arbeit "Einfachheit" und "(ontologische) Sparsamkeit" trennen und eine voneinander unabhängige Charakterisierung beider Merkmale geben.

\subsubsection{Einfachheit}

Unter Einfachheit möchte ich fortan Folgendes verstehen: Theorien sind einfach, wenn sie ohne übermäßige Mühen kognitiv zugänglich sind und dadurch der Inhalt verstanden werden kann. Eine Einfachheit bedeutet auch, dass Theorien rascher verstehbar sind. Gleichsam sind die Theorien übersichtlich dargestellt und der Inhalt ist eindeutig erfassbar und bedarf keiner übermäßigen Interpretation. Das Urteil, ob eine Theorie einfach ist, treffen zwei Gruppen von Personen. Einerseits die Gruppe von fachlich versierten und rationalen Personen, andererseits die Gruppe von durchschnittlichen Personen mit einem durchschnittlichen themenbezogenen Wissen. Ob nun eine fachlich besonders informierte und rationale Person oder ein Laie 
heranzuziehen ist, ist davon abhängig, ob das Einfachheitsprinzip beispielsweise in einem fachlichen Spezialbereich Anwendung finden soll oder in alltäglichen Bereichen.

Diese Punkte sollen die ersten Anker einer Einfachheit darstellen, ein Hinzufügen weiterer Spezifizierungen und Beispiele ist somit möglich. Dadurch können wir mit einem Grundverständnis in die weiteren Analysen gehen und im Zuge der konkreten Anwendung des Prinzips der Einfachheit in der Physikalismusdebatte immer weiter ein tieferes Verständnis erlangen. Wenn man so will, dann kann man die hier eingeführte Charakterisierung der Einfachheit als eine hinreichend klare Definition des Begriffes "Einfachheit" verstehen, um innerhalb der Physikalismusdebatte in eine Diskussion und Argumentation treten zu können, die jedoch auch offen für Erweiterungen ist:

(Einfachheit) T1 ist einfacher als T2 genau dann, wenn der Inhalt von T1 kognitiv zugänglicher oder rascher verstehbar oder übersichtlicher dargestellt oder eindeutiger erfassbar ist als der Inhalt von T2.

Ich möchte als Beispiel eine empirische Verteilung von bestimmten Messwerten einführen, die in eine mathematische Formel gebracht werden sollen. Diese Messwerte liegen derart in einem Koordinatensystem, dass sie beinahe linear angeordnet, aber doch hin und wieder etwas weiter weg von der gedachten geraden Linie sind. Dabei erinnert die Verteilung der Messwerte aufgrund einiger weiter weg liegenden Datenpunkte etwas an eine Wellenform, doch auch das ist nicht ganz richtig, weil die Ausbuchtungen äußerst minimal sind. Nichtsdestotrotz benötigt man eine mathematische Formel, welche die vorliegende empirische Verteilung am besten beschreibt. Einerseits beschreibt ein polynomer Zusammenhang die Daten ausgezeichnet, andererseits beschreibt ein linearer Zusammenhang die Daten in gleichem Maße ausgezeichnet. Für welche Art des Zusammenhangs soll man sich entscheiden? Bei Anwendung des Prinzips der Einfachheit fällt die Entscheidung auf den linearen Zusammenhang, denn wenn alle Eigenschaften beider Arten der Zusammenhänge dieselben sind, dann gibt die Einfachheit den Ausschlag. Ein linearer Zusammenhang ist ein Paradebeispiel, bei dem alle vier disjunktiven Zuschreibungen zur Einfachheit erfüllt sind: Er ist kognitiv zugänglicher, rascher verstehbar, übersichtlicher dargestellt und eindeutiger erfassbar als ein polynomer Zusammenhang.

\subsubsection{Sparsamkeit}

Ich möchte mich nun dem Kriterium der "Sparsamkeit" zuwenden und sie durch Beispiele einführen. Theorien sind auf eine Leseart sparsam, wenn sie zur Erklärung nur so wenig wie möglich und so viel wie unbedingt notwendig als existierend annehmen, seien es Entitäten, Eigenschaften, Relationen oder dergleichen. In diesem Gewand handelt es sich um eine 
ontologische Sparsamkeit und ist in der philosophischen Literatur oftmals als Ockhams Rasiermesser bekannt. In einer anderen Leseart sind Theorien sparsamer, wenn sie in ihren Erklärungen nur so wenig Sprachliches wie möglich und unbedingt notwendig verwenden, seien es Sätze, Wörter, Ausdrücke oder dergleichen. Diese Art von Sparsamkeit möchte ich begriffliche Sparsamkeit nennen. Auch bei diesem Kriterium der Sparsamkeit sind weitere Ergänzungen, Erweiterungen und Spezifizierungen nicht ausgeschlossen und die hier eingeführte Charakterisierung soll als eine hinreichend klare Definition des Begriffes "Sparsamkeit" verstanden werden, um auch damit in sinnvolle Diskussionen und Argumentationen treten zu können:

(Sparsamkeit) T1 ist sparsamer als T2 genau dann, wenn der Inhalt von T1 i) weniger Entitäten, Eigenschaften, Relationen und dergleichen beinhaltet (= ontologische Sparsamkeit) und/oder ii) durch weniger Sätze, Wörter, Ausdrücke und dergleichen erfasst wird (= begriffliche Sparsamkeit) als der Inhalt von T2.

Um beispielsweise die Entstehung von Regen in Wolken zu erklären, werden einige grundlegende physikalische Gesetze und bestimmte Umgebungsfaktoren benötigt, die gemeinsam zu Regen führen. Als alternative Theorie dazu kann eine Gottestheorie eingeführt werden, in der zu den relevanten grundlegenden Gesetzen und bestimmten Umgebungsfaktoren noch zusätzlich ein Regengott postuliert wird, der an der Entstehung von Regen beteiligt ist, weil eben nur der Regengott die physikalischen Gesetze durch seinen Willen aktivieren kann. Beide Theorien erklären das Phänomen des Regens gleich gut, doch für welche der beiden Theorien soll man sich entscheiden? Bei Anwendung des Prinzips der ontologischen Sparsamkeit fällt die Entscheidung auf die erste der beiden Theorien, weil sie ohne die Postulierung eines Gottes auskommt.

Betrachten wir weiters eine Theorie über Junggesellen. Die erste Theorie beschreibt, warum Junggesellen gerne in Zweiraumwohnungen leben und warum Junggesellen einen großen Freundeskreis haben. Die zweite Theorie beschreibt ebenso, warum Junggesellen gerne in Zweiraumwohnungen leben, aber auch gleichzeitig warum unverheiratete Männer einen großen Freundeskreis haben. Selbstverständlich haben beide Theorien denselben Erklärungsgehalt, weil Junggesellen nichts anderes als unverheiratete Männer sind. Beide Theorien erklären das Leben von Junggesellen bzw. unverheirateten Männern gleich gut, doch für welche der beiden Theorien soll man sich entscheiden? Bei Anwendung des Prinzips der 
begrifflichen Sparsamkeit fällt die Entscheidung auf die erste der beiden Theorien, weil sie ausschließlich den Begriff "Junggeselle" verwendet und nie den Begriff "unverheirateter Mann"35.

\subsubsection{Ein rationales Vorziehen von Theorien}

Ich möchte hier meine Überlegungen aus dem Bereich der Evolutionstheorie heraus starten und zeigen, warum einfachere und sparsamere Theorien mit größerer Wahrscheinlichkeit wahr sind als komplexere und weniger sparsame Theorien. Da ich die Einfachheit und Sparsamkeit als eigenständige Kriterien definiert habe, werde ich sie auch nachfolgend separat behandeln. Trotz der Eigenständigkeit hängen auch beide Kriterien miteinander zusammen, nämlich derart, dass zum einen bei gegebener Einfachheit von T1 im Vergleich zu T2 auch gleichzeitig die Sparsamkeit von T1 im Vergleich zu T2 größer oder gleich ist, aber niemals niedriger. Zum anderen ist bei gegebener Sparsamkeit von T1 im Vergleich zu T2 auch gleichzeitig die Einfachheit von T1 im Vergleich zu T2 größer oder gleich, aber niemals niedriger. Das bedeutet, dass die Einfachheit nie gegen eine Sparsamkeit arbeitet oder umgekehrt.

Kritiker könnten jedoch folgenden Einwand vorbringen und auf eine vermeintliche Unvereinbarkeit zwischen Einfachheit und ontologischer Sparsamkeit hindeuten. Die Entwicklung des Menschen ausschließlich evolutionsbiologisch darzustellen, ist schwierig zu verstehen. Es ist einfacher zu verstehen, dass Gott den Menschen durch ständige Schöpfungsakte geformt und die Naturgesetze laufend angepasst hat, damit wir in den Naturwissenschaften diese Evolution erforschen können. Dabei erliegen wir der Illusion, dass die Evolution durch eine natürliche Auslese geschieht, obwohl hinter den Kulissen Gott die Fäden zieht. Deshalb muss ich nur verstehen, dass Gott sich um seine Schöpfung kümmert. Als Replik kann entgegnet werden, dass für die Beurteilung der Einfachheit nicht eine durchschnittliche Person heranzuziehen ist, sondern ein objektiver, rationaler Beurteiler mit hinreichender themenspezifischer Fachexpertise. Ob die Einführung eines Gottes zusätzlich zur Evolutionstheorie einfacher ist, ist unabhängig von religiösen Vorstellungen, von Werthaltungen und vermeintlichen Interessenskonflikten zu beantworten. Ein objektiver, rationaler Fachexperte würde hier aus der Biologie kommen und als Maßstab heranzuziehen sein. Sein Urteil würde sein, dass die klassische Evolutionsbiologie einfacher ist als die Gottes-Theorie, denn sie ist kognitiv zugänglicher, rascher verstehbar, übersichtlicher dargestellt und eindeutiger erfassbar. Hinsichtlich der ontologischen Sparsamkeit ist die Evolutionstheorie im Vergleich zur GottesTheorie sparsamer, weil sie eben ohne einem Extra in der Form eines Gottes auskommt.

Oder betrachten wir einen weiteren möglichen Einwand von Kritikern, um eine Unvereinbarkeit zwischen Einfachheit und begrifflicher Sparsamkeit zu zeigen. Die ausmultiplizierte Form der Potenz einer Summe als $a^{2}+2 a b+b^{2}$ ist einfacher zu verstehen als

\footnotetext{
35 Umgelegt auf die Diskussion in der Philosophie des Geistes schreibt Gole (2008a) provokativ (S. 127): "Let us make the point clear: scientific psychological theories are not the same as poems. Poems need a lot of fancy words, but scientific theories just do not".
} 
die zusammengefasste Form $(a+b)^{2}$. Ein hoher Abstraktionsgrad mit weniger Begriffen ist nicht notwendigerweise einfacher. Als Replik kann auch hier wieder gebracht werden, dass für die Beurteilung der Einfachheit keine durchschnittliche Person heranzuziehen ist, sondern jemand mit einer mathematischen Fachexpertise. Ob eine stark zusammengefasste mathematische Formel schwieriger zu verstehen ist als eine maximal ausmultiplizierte Form, ist unabhängig von den eigenen Vorlieben und den eigenen mathematischen Kenntnissen zu sehen. Ein objektiver, rationaler Fachexperte würde hier aus der Mathematik kommen und als Maßstab heranzuziehen sein. Sein Urteil würde sein, dass die abstrakte Formel einfacher ist als die ausmultiplizierte Formel, denn sie ist kognitiv zugänglicher, rascher verstehbar, übersichtlicher dargestellt und eindeutiger erfassbar. Hinsichtlich der begrifflichen Sparsamkeit ist die abstrakte Formel im Vergleich zur ausmultiplizierten Formel sparsamer, also jedenfalls nicht weniger sparsam.

Nach diesen Klarstellungen zum Zusammenhang zwischen der Einfachheit und der Sparsamkeit gilt es nun zu zeigen, dass unter der Gültigkeit bestimmter Beobachtungen (B) die einfachere bzw. sparsamere Theorie, unter Beachtung des dargelegten Zusammenhanges zwischen der Einfachheit und Sparsamkeit, eine größere Wahrscheinlichkeit hat, wahr zu sein. Einfachere bzw. sparsamere Theorien sind dann wahr, wenn einer der folgenden zwei Bedingungen erfüllt ist. Erstens, unter der Voraussetzung der Beobachtungen B ist die Wahrscheinlichkeit größer, dass einfache Theorien (E) wahr sind im Vergleich zu nichteinfachen Theorien ( $\neg \mathrm{E}$ ) und gleichzeitig die Wahrscheinlichkeit größer oder gleich ist, dass sparsame Theorien (S) wahr sind im Vergleich zu nicht-sparsamen Theorien ( $\neg S)$. Zweitens, unter der Voraussetzung der Beobachtungen B ist die Wahrscheinlichkeit größer, dass sparsame Theorien (S) wahr sind im Vergleich zu nicht-sparsamen Theorien ( $\neg S$ ) und gleichzeitig die Wahrscheinlichkeit größer oder gleich ist, dass einfache Theorien (E) wahr sind im Vergleich zu nicht-einfachen Theorien ( $\neg E)$. Formal lässt es sich so ausdrücken:

$$
\text { (W) } \begin{aligned}
& P((E \mid B)>P(\neg E \mid B)) \& P((S \mid B) \geq P(\neg S \mid B)) \text { oder } \\
& P((S \mid B)>P(\neg S \mid B)) \& P((E \mid B) \geq P(\neg E \mid B))
\end{aligned}
$$

Ziehen wir als Beispiel das Verhalten eines Pfaus heran. Sobald ein Weibchen in der Nähe ist, fächert das Männchen seine wunderschönen, bunten und glänzenden Federn auf. Das sind unsere Beobachtungen des Pfau-Verhaltens. Auf Basis dieser Beobachtung wollen wir eine Theorie formulieren, warum das Männchen sein Federkleid in der Gegenwart von Weibchen präsentiert. Wir setzen uns also auf einen Baumstumpf in sicherer Entfernung des Pfaus, zücken unser Notizbuch und beginnen, mögliche wahre Theorien aufzuschreiben. Als erste Theorie fällt uns ein, dass der Pfau deshalb ein Rad schlägt, damit er das einfallende Licht reflektieren kann, um die Weibchen bei der Futtersuche zu unterstützen. Im Rad des Pfaus existieren viele kleine Käfer mit einem außerordentlich glatten Panzer, die in symbiotischer Weise mit inm zusammenleben und immer dann, wenn Licht einfällt und sich die 
Umgebungstemperatur auch nur eine kleine Spur erhöht, in ihrem Panzer verschwinden. Sobald sie im Panzer verschwunden sind, verhalten sie sich ruhig und ermöglichen damit eine Reflexion des Sonnenlichtes über ihren Panzer. Neben dieser "Käfer-Theorie" fällt uns etwas Weiteres ein. Der Pfau schlägt deshalb ein Rad, weil er ganz bestimmte Fressfeinde dadurch abschreckt. Diese Fressfeinde sehen nicht nur dasjenige Farbspektrum, das wir als Menschen sehen, sondern darüber hinaus noch Farben im ultravioletten Bereich. Genau dieses Farbspektrum schreckt sie schlussendlich ab, Pfaue zu fressen. Diese "Fressfeinde-Theorie" ist nun die zweite Erklärung für das beobachtbare Verhalten. Schließlich notieren wir eine dritte Theorie, die auch tatsächlich die korrekte ist. Pfaue schlagen deshalb ein Rad, weil es zu ihrem Balzverhalten gehört, um die Gunst der Hennen zu ergattern. Vergleichen wir nun diese "Balz-Theorie" mit den anderen beiden vorgeschlagenen und frei erfundenen Theorien.

Im Vergleich der Balz-Theorie mit der Käfer-Theorie ist erstere ontologisch sparsamer, weil keine Käfer mit außerordentlich glatten Panzern postuliert werden müssen. Ebenso ist erstere Theorie einfacher, weil sie durch die überschaubare und kurze Erklärung des Verhaltens kognitiv zugänglicher ist, rascher verstehbar, übersichtlicher dargestellt und eindeutig erfassbarer ist. Ein ähnliches Bild ergibt sich bei Vergleich mit der Fressfeinde-Theorie. Die Balz-Theorie ist auch hier ontologisch sparsamer, weil kein Farbsehvermögen im ultravioletten Bereich bei bestimmten Fressfeinden postuliert werden muss. Gleichsam gewinnt sie in allen vier Spezifikatoren der Einfachheit das Rennen gegen die Fressfeinde-Theorie, eben aufgrund der überschaubaren und kurzen Erklärung des Pfau-Verhaltens. Die Bedingungen von (W) sind somit erfüllt.

So wie es sich beim Radschlagen des Pfaus verhält, verhält es sich auch bei vielen anderen Beobachtungen aus der Natur, wo einer einfachen und ontologisch sparsamen Theorie wenig einfache und wenig sparsame konkurrierende Theorien gegenübergestellt werden. Letztendlich haben sich über die Wissenschaftsgeschichte hinweg immer die einfacheren und sparsameren Theorien zumindest als vorläufig wahr entpuppt. Dabei ist nicht von Relevanz, ob die als vorläufig für wahr gehaltene Theorie auch tatsächlich immer und ewig, komme was wolle, für wahr gehalten werden muss. Nein, es ist vielmehr von Relevanz, dass deren Wahrscheinlichkeit, wahr zu sein, höher ist als die Wahrscheinlichkeit der Konkurrenztheorien. Genau das ist es, was in (W) ausgedrückt ist und durch Abstraktion des Pfau-Beispiels als rationaler Wegweiser verwendet werden kann, um mehrere Theorien miteinander zu vergleichen. Man gelangt zu (W) durch einen Induktionsschluss, indem aus der bisherigen Wissenschaftsgeschichte immer die einfacheren und sparsameren Theorien das Rennen gemacht haben und man deshalb darauf schließt, dass es auch zukünftig so sein wird. Gleichzeitig wird von naturwissenschaftlichen Fragestellungen auf metaphysische Fragestellungen generalisiert ${ }^{36}$. Selbstverständlich kann stets gegen einen Induktionsschluss

${ }^{36}$ Siehe Bradley (2018), der in seinen Analysen zum Schluss kommt: "So there seems to be no principled reason why the considerations applied in scientific controversies should not also be applied in philosophical controversies" 
argumentiert werden, dass dieser generell unvernünftig sei, weil er nicht notwendigerweise wahr ist. Darauf möchte ich antworten, dass dies vielleicht der saure Apfel ist, in den man beißen muss. Nicht deshalb, weil es einem gut schmeckt, sondern weil es keine andere Option gibt, wenn man sich eines Induktionsschlusses bedient. Doch was ist die Alternative? Entweder ich akzeptiere die vorläufige Gültigkeit des Induktionsschlusses in (W), bis eine andere bewährte Richtlinie zur Entscheidung zwischen Theorien dessen Platz einnimmt, oder ich lehne den Induktionsschluss mit (W) ab und ich stehe ohne ein systematisches Entscheidungsprozedere da. Unter diesen Umständen entscheide ich mich für die vorläufige Akzeptanz von (W) und entwickle auf Basis dessen weiter, was es für das Bewusstseinsproblem in der Philosophie des Geistes bedeutet.

\subsection{Von Supervenienz zur Typen-Identität}

Im letzten Kapitel wurde der Supervenienzphysikalismus, und damit auch alle darauf aufbauenden physikalistischen Positionen, vor Hawthornes Blocker-Problem gerettet. Ob nun die Supervenienz als minimaler Physikalismus eine notwendige Bedingung für die Formulierung des Physikalismus ist oder sogar hinreichend ist, bleibt offen. Ebenso bleibt die Frage offen, warum letztendlich die Supervenienz als Relation zwischen dem Physikalischen und Mentalen besteht. Jackson (1998a), Chalmers (1996) und Lewis (1994) setzen beispielsweise ihre Supervenienzformulierungen mit dem Physikalismus gleich, sie legitimieren die Supervenienz somit als eine hinreichende Bedingung für den Physikalismus. Andere Vertreter geben der Supervenienz typischerweise noch weitere Aspekte hinzu (siehe die einzelnen Positionen unter Kapitel 3), welche die Supervenienz somit nur als notwendige Bedingung akzeptieren.

Ein wesentlicher Einwand gegen den Supervenienzphysikalismus stammt von Kim (1993). Wenn wir uns auf die globale Supervenienzthese (Sglobal) beziehen, dann folgt über mögliche Welten hinweg bei gleicher A-Aufmachung auch dieselbe B-Aufmachung. Hier aber, so Kim, ist noch nicht gesichert, dass die B-Aufmachung auch tatsächlich physikalisch ist. So ist (Sglobal) beispielsweise auch mit dem Epiphänomenalismus vereinbar, weil die Wahrheit von (Sglobal) auch mit der Wahrheit von kausal impotenten mentalen Eigenschaften vereinbar ist. Sie sollten jedoch nicht vereinbar sein. Dieses Problem führte schließlich zu weiteren Anpassungen der Supervenienzthese und Formulierungen wie die von Jackson (1998a) sind nicht mehr für diese Art von Einwänden anfällig und Jacksons Supervenienzphysikalismus ist nicht mit der Wahrheit des Epiphänomenalismus vereinbar.

(S. 3065-3066), oder auch Brenner (2017): "Rather, I endorse the much more modest claim that, if theoretical simplicity is truth conducive in science, then it is truth conducive in metaphysics as well" (S. 2689). Hier ist zu beachten, dass Bradley und Brenner das Merkmal der Einfachheit nicht in gleicher Art und Weise wie ich verwenden, sondern darunter auch eine ontologische Sparsamkeit subsummieren. Wenn ich also von den beiden Merkmalen der Einfachheit und Sparsamkeit spreche, sprechen andere Autoren von einem einzigen Merkmal, das inhaltlich meine beiden Merkmale einschließt. 
Was jedoch Jacksons Supervenienzphysikalismus und ähnliche Formulierungen nicht liefern, ist eine Erklärung, warum, erstens, von einem gemeinsamen Auftreten von $A$ und $B$ eine Supervenienzrelation zwischen A und B folgt und, zweitens, wie diese Supervenienzrelation ausschließlich physikalistisch erklärt und expliziert werden kann. Dieses Problem hat Horgan (1993) formuliert und gleichzeitig eine Lösung vorgeschlagen. Um diesem Problem gerecht zu werden, muss sich nach Horgan die Relation der Supervenienz in eine Relation der Superdupervenienz entwickeln. Eine Möglichkeit, wie aus der Supervenienzrelation eine Superdupervenienzrelation wird, wäre eine Identitätsrelation ${ }^{37}$. Sowohl Kim als auch Horgan stimmen überein, dass durch die Identität von A und B klar wird, warum beides gemeinsam auftritt, denn sie sind ein und dasselbe. Ebenso wird dadurch klar, warum die in eine Identitätsrelation kollabierte Supervenienz physikalistisch einfach erklärt und expliziert werden kann, weil nämlich beide ein und dasselbe sind und $B$ auf $A$ reduziert wurde.

Wenn ich von Identität spreche, dann meine ich eine Typenidentität zwischen A und $B$ bzw. zwischen dem physikalischen und mentalen Typus. Die Diskussion aus Kapitel 3 aufgreifend, ist der Ablehnungsgrund für eine Token-Identität die Vereinbarkeit mit einem Dualismus auf Typenebene. Um jedoch als eine physikalistische Theorie zu gelten, darf kein ontologischer Dualismus wie auch immer geartet mit der Wahrheit des Physikalismus vereinbar sein. Wenn ich nachfolgend von einer Identitätstheorie spreche, dann meine ich damit auch immer eine Typen-Identitätstheorie. Die wesentliche Frage an dieser Stelle ist nun, warum eine Identitätsrelation angenommen werden soll und keine andere passende Relation konkurrierender physikalistischer Positionen.

\subsubsection{Die Identität als beste Alternative}

Sowohl die Identitätstheorie als auch der Supervenienzphysikalismus sind Theorien über gewisse Beobachtungsdaten und beide Theorien liefern Erklärungen für das Auftreten der Phänomene in Frage. Aus diesem Grunde sind beide in den wesentlichen Dingen, die sie als Bewusstseinstheorien ausmachen, gleichzuhalten. Um sich argumentativ für eine Theorie entscheiden zu können, möchte ich als Erstes unser Kriterium der Einfachheit betrachten. Die Identitätstheorie ist im Vergleich zum Supervenienzphysikalismus kognitiv zugänglicher, rascher verstehbar, übersichtlicher dargestellt und eindeutiger erfassbar. Alles, was es braucht, ist das Mentale und das Physikalische als ein und dasselbe zu sehen. Hinsichtlich der Sparsamkeit präsentiert sich die Identitätstheorie als ontologisch sparsamer. Das ist insbesondere deshalb der Fall, weil auf eine altbekannte Relation, nämlich die der Identität, zurückgegriffen werden kann und keine neue Relation einer Supervenienz ontologisch eingeführt werden muss.

\footnotetext{
37 So fasst auch Tiehen (2018) in seinem Übersichtsartikel zum Physikalismus die Debatte zu einer Superdupervenienz folgendermaßen zusammen (S. 539): "In response to this line of criticism, several philosophers in recent years have sought some stronger and more explanatory relation than supervenience in order to analyse nothing over and above".
} 
Als nächste Bewusstseinstheorie aus Kapitel 3 ist der Behavorismus zu nennen. Dieser ist im Vergleich zur Identitätsthese auszuschließen, weil er den fatalen Fehler begeht, jegliche bedeutsamen psychischen Faktoren in der Generierung von Verhalten bzw.

Verhaltensdispositionen nicht miteinzubeziehen. Keine Interpretation oder Bewertung der Umweltreize spielt eine Rolle, sondern nur der Reiz an sich, der eine typische Reaktion nach sich zieht. Der Identitätstheoretiker hingegen bezieht Interpretationen, Bewertungen und dergleichen als mentale Zustände mit ein, zwar als nichts anderes als etwas Physikalisches, doch er würdigt sie. Hier gibt weder die Einfachheit noch die Sparsamkeit den Ausschlag, sondern die Grundpfeiler des Behaviorismus.

Weiters ist der Repräsentationalismus/Intentionalismus im Vergleich zur Identitätstheorie auszuschließen, weil in einem ersten Schritt alle Qualia auf intentionale Zustände reduziert werden, um in einem zweiten Schritt Intentionalität auf Gehirnzustände zu reduzieren. Der erste Schritt ist ein diskussionswürdiger Punkt und der zweite Schritt hat das ursprüngliche Problem lediglich verschoben: Denn nun wird gefragt, wie Intentionalität bzw. Repräsentationsinhalte einen Platz in der Ontologie des Physikalisten finden. Hingegen klammert der Identitätstheoretiker den Umweg über eine "Intentionalisierung" bei der Reduktion von Qualia aus und identifiziert sie gleich mit einer physikalistischen Basis. Das Einführen des (zweifelhaften) ersten Schrittes innerhalb des Lagers des Repräsentationalismus bzw. Intentionalismus und die darauffolgende Verlagerung des ursprünglichen Problems geht entgegen einer Einfachheit, weil dadurch nichts Wesentliches zur Lösung des Problems beigetragen wurde. Dabei ist unerheblich, ob es sich um einen schwachen oder starken bzw. um einen engen oder weiten Repräsentationalismus handelt. Der Repräsentationalist könnte kontern, dass sein Ansatz jedoch ontologisch sparsamer sei, weil in seiner Theorie nur von intentionalen Zuständen gesprochen werden muss, aber nicht auch zusätzlich von Qualia. Hier möchte ich entgegnen, dass dieses Kriterium nur dann greift, wenn auch die Reduktion von qualitativen Zuständen auf intentionale Zustände gezeigt wurde. Doch genau dieser Schritt ist zweifelhaft und schürt Kritik.

Beim Vergleich des Fundierungsphysikalismus mit der Identitätstheorie zieht auch dieser den Kürzeren, weil die Fundierungsrelation als eine neue, fundamentale Relation verstanden wird, die wiederum die Ontologie um eine weitere Relation erweitert. Eine Erweiterung der Ontologie geht entgegen einer ontologischen Sparsamkeit und eine Erweiterung ist in diesem Fall auch nicht notwendig, weil auf die bestehende Relation der Identität zurückgegriffen werden kann. Den Ausführungen des letzten Kapitels folgend ist zudem der Status der Fundierungsrelation als eine eigene Relation noch gar nicht klar, weil einige Kritiker die Fundierungsrelation in eine der bereits bekannten Relationen aufgelöst sehen.

Hinsichtlich des Vergleiches des Funktionalismus mit der Identitätstheorie möchte ich zum einen in aller Kürze zwischen funktionalistische Typen und Tokens unterscheiden und zum anderen zwischen einem Funktionalismus erster Ordnung und einem zweiter Ordnung 
differenzieren. Wie bereits mehrmals von mir dargelegt, ist eine Bewusstseinstheorie, die ausschließlich mit Token-Identitäten arbeitet, nicht davor gefeit, dass auf Typenebene ein Dualismus eingeführt wird. Dabei ist es unerheblich, ob auf speziesspezifische Gehirnprozesse, Repräsentationen, Verhaltensdispositionen oder funktionalistisch-kausale Rollen auf TokenEbene reduziert wird. Da somit jegliche Token-Identitätstheorie ausgeschlossen ist, ist auch eine funktionalistische Token-Identitätstheorie ausgeschlossen. Im Kontrast dazu wäre die Reduktion eines mentalen Typus auf einen funktionalistischen Typus ein Paradebeispiel einer Identitätstheorie, so wie es Identitätstheoretiker der ersten Stunde vorgelegt haben (Smart, 1959; Lewis, 1966; Armstrong, 1968). Ob jedoch seit dem Vorlegen des Arguments der multiplen Realisierbarkeit Funktionalisten als funktionalistische Typen-Identitätstheoretiker gelten wollen und ob es überhaupt mit den Kernaussagen des Funktionalismus vereinbar ist, bezweifle ich. Die gesamte Motivation, ein Funktionalist zu sein, wird durch die Annahme einer Typen-Identität zwischen dem Mentalen und dem Physikalischen untergraben. Obwohl es eine prinzipielle Lösung bleibt, ist sie gegenwärtig eine Außenseiterposition. Überlegungen zur Einfachheit oder Sparsamkeit geben erst bei Betrachtung eines Funktionalismus erster Ordnung oder zweiter Ordnung den Ausschlag.

Beim Vergleich der Identitätstheorie mit dem Funktionalismus zweiter Ordnung, in dem beispielsweise Schmerz nichts anderes ist als ein Zustand, eine bestimmte neuronale Eigenschaft zu haben, welche die Schmerz-Rolle einnimmt, ist die Identitätstheorie einfacher. Der Grund ist, dass der mentale Zustand mit dem physikalischen Zustand direkt identisch ist und nicht den Umweg über eine Zwischenstation, nämlich in einem Zustand zu sein, eine bestimmte neuronale Eigenschaft zu haben, gehen muss. Aus demselben Grund ist die Identitätstheorie auch ontologisch sparsamer, weil kein zusätzlicher Zustand als Zwischenstation eingeführt werden muss. Bei Vergleich der Identitätstheorie mit dem Funktionalismus erster Ordnung auf Typenebene, in dem beispielsweise Schmerz nichts anderes als eine bestimmte neuronale Eigenschaft ist, welche die Schmerz-Rolle einnimmt, ist diese Spielart des Funktionalismus wiederum ein Paradebeispiel einer Identitätstheorie. Also ist zusammengefasst ein TypenFunktionalismus erster Ordnung eine gangbare Version, wie eine Identitätstheorie weiter ausformuliert werden könnte.

\subsubsection{Gegen das Argument der multiplen Realisierbarkeit}

Hinsichtlich der Gründe für die breite Akzeptanz des Funktionalismus und damit zusammenhängend Token-Identitäten zwischen dem Physikalischen und Mentalen war ab den 60er-Jahren das Argument der multiplen Realisierbarkeit der Hauptbeweggrund, eine TypenIdentitätstheorie abzulehnen. Seien es Frösche, Tintenfische oder Menschen, für deren Schmerzerlebnisse existiert nicht eine gemeinsame physikalische Basis, welche das Schmerzerlebnis funktional-kausal realisiert, sondern es existieren jeweils verschiedene 
interindividuelle Realisierungen. Gleichzeitig sind innerhalb einer Spezies und sogar innerhalb eines Individuums über verschiedene Zeitpunkte hinweg verschiedene intraindividuelle Realisierungen vorhanden. Die Realisierung meines Schmerzerlebnisses am Vormittag ist nicht notwendigerweise dieselbe wie am Nachmittag. Doch haben die Kernüberlegungen des Arguments der multiplen Realisierbarkeit auch noch nach 50 Jahren Gültigkeit und sind sie nach wie vor schlüssig im Lichte der neueren naturwissenschaftlichen Erkenntnisse? Ich möchte diese Frage verneinen und zeigen, warum das Argument der multiplen Realisierbarkeit bei genauerer Analyse verschwindet und den Weg für eine Typen-Reduktionstheorie freigibt.

Ich nehme die Analyse von Bechtel und Mundale (1999, S. 202) als Ausgangpunkt, welche die genaue Art und Weise der Betrachtungsform der physikalischen und mentalen Vorgänge als Quelle der Illusion einer vermeintlichen multiplen Realisierbarkeit heranziehen:

Thus, one diagnosis of what has made the multiple realizability claim as plausible as it has been is that researchers have employed different grains of analysis in identifiying psychological states and brain states, using a coarse grain to identify psychological states and a fine grain to differentiate brain states. Having invoked different grains, it is relatively easy to make a case for multiple realization. But if the grain size is kept constant, then the claim that psychological states are in fact multiply realized looks far less plausible.

Es ist somit die konsistente Körnigkeit der Betrachtung der physikalischen Ebene und der mentalen Ebene ausschlaggebend, die innerhalb der jeweiligen feinkörnigen oder grobkörnigen Betrachtungsweise das Argument der multiplen Realisierbarkeit verschwinden lassen. Nehmen wir Furcht als Beispiel bei einer grobkörnigen Betrachtung. In diesem Fall wäre bei Tintenfischen, Fröschen und Menschen der mentale Zustand von Furcht vorhanden, wenn sich alle drei Spezies von einem Fressfeind wegbewegen. Der mentale Zustand der Furcht hat hier die Funktion, das eigene Überleben zu sichern. Weiters ist Furcht in dieser allgemeinen Form durch eine Aktivität im Gehirn der jeweiligen Spezies realisiert. Dabei zählt hier als Gehirnaktivität jegliche Aktivierung, wie auch immer diese sein mag. Auf dieser sehr grobkörnigen und abstrakten Ebene, ohne jegliche Spezifitäten zu betrachten, ist derselbe mentale Zustand mit demselben Realisierer gegeben und kein Anflug einer multiplen Realisierbarkeit kommt auf.

Nun nehmen wir Furcht als Beispiel bei einer feinkörnigen Betrachtung. Dann müsste man ein Furchterlebnis der Person X1, zugehörig zur Spezies Mensch, zum Zeitpunkt t1 betrachten, der in genau ihrem Gehirn mit dem physikalischen Zustand P1 in der Region R1 zum entsprechenden Zeitpunkt realisiert ist. Das wird verglichen mit einem Furchterlebnis des Tieres T1, zugehörig zur Spezies Tintenfisch, zum Zeitpunkt t1, das nun genau in seinem Gehirn mit dem physikalischen Zustand P1 in der Region R1 des entsprechenden Tintenfisches zum 
entsprechenden Zeitpunkt realisiert ist. Furcht in dieser ganz konkreten individuierten Situation und eine Aktivität des Gehirns in einem ganz konkreten lokalisierten und molekularen Zustand ist voll mit Spezifitäten. Doch was hier nicht auftaucht, ist ein Anflug einer multiplen Realisierbarkeit, weil verschiedene Furchttypen über die Spezies und individuierten Situationen hinweg in einem jeweils verschiedenen Gehirnzustand realisiert sind. Der Grund dafür ist, dass verschiedene Spezies über verschiedene Zeitpunkte geringfügig andere mit dem Furchterlebnis verbundene Aktivitäten setzen und damit die Funktion der Furcht verschieden ist. Ein Mensch wird nach dem Wegbewegen womöglich zum Reflektieren beginnen, wie er seine emotionale Reaktion kontrollieren kann, ein Frosch würde wegspringen und sich im Laub tarnen und ein Tintenfisch würde nach der Absonderung von Tinte schnell davonschwimmen. Es sind deshalb verschiedene biologische Substrate vorhanden, weil auch verschiedene Funktionen, Rollen und dergleichen als mentale Zustände realisiert werden. Damit ist auch hier kein Anflug einer multiplen Realisierbarkeit zu finden, weil nicht dieselbe Funktion realisiert wird, sondern verschiedene Funktionen auch verschieden realisiert werden.

Die Illusion einer multiplen Realisierbarkeit würde nur dann entstehen, wenn, so wie Putnam (1967) es getan hat, ein mentaler Zustand wie Furcht unter einer grobkörnigen Betrachtung über alle Spezies und Situationen hinweg mit den verschiedenen Realisierern der einzelnen Spezies und Situationen zu vergleichen. Hier könnte man fälschlicherweise annehmen, dass Furcht multipel realisiert wird. Doch ein Konstanthalten der Betrachtungsebene in einheitlich grob- oder feinkörniger Art und Weise löst das Problem auf.

Bechtel (2012) erweiterte seine ursprünglichen Gedanken anhand der zirkadianen Uhr, die bei Menschen und vielen anderen Spezies auch für den Schlaf-/Wachrhythmus verantwortlich ist. Obwohl es über einige Spezies hinweg geringfügige Unterschiede in der Realisierung der zirkadianen Uhr zu geben scheint, kontert Bechtel auf zwei Weisen. Zum einen greift er zurück auf die Körnigkeit der beiden Betrachtungsebenen, sodass bei der feinkörnigen Betrachtung durch die verschiedenen biologischen Unterschiede eigentlich auch verschiedene zirkadiane Uhren realisiert werden und dadurch der Vorwurf einer multiplen Realisierbarkeit entkräftet wird. Zum anderen greift er seine Idee einer unwesentlichen Modifikation der verschiedenen zirkadianen Uhren auf, die jedoch aufgrund der gemeinsamen Abstammung im Laufe der Evolution eine wesentliche Übereinstimmung in der Organisation und Funktion aufweisen. Dabei bleibt die Kernfunktion der zirkadianen Uhren dieselbe, nämlich das Regeln des Schlaf-/Wachrhythmuses (S. 61-62):

Distinguishable mechanisms only generate the same phenomenon when a much coarser grain is employed for the phenomenon than the mechanism. By employing as restrictive a grain for the phenomena as for the mechanisms in cases where the mechanisms differ, the phenomena can be distinguished as well. [...] But the fact that they share a common organization may indicate 
functional homology (same organization as a result of common descent) and if so provide a basis for treating all circadian clocks as conserved and hence members of the same type. [...] Underlying this is an identity claim between a type of clock mechanism and the type of circadian phenomenon.

Durch diese Sichtweise gehören alle zirkadianen Uhren zum selben Typus "zirkadiane Uhr", welcher identisch zum Typus "Schlaf-/Wachrhythmus" ist. Bechtel (2012) zielt hier ganz klar auf eine Typenidentität ab, trotz geringfügiger Variabilitäten innerhalb der Mechanismen der verschiedenen zirkadianen Uhren, und entkräftet somit auf diese Art und Weise den Vorwurf einer multiplen Realisierbarkeit.

Ich möchte diese Idee einer funktionalen Homologie zwischen verschiedenen Spezies aufgreifen und Bechtels Argument auf die mentalen und physikalischen Zustände erweitern. Homologe Strukturen sind in der Biologie weitestgehend übereinstimmende Strukturen, die sich in verschiedenen Spezies im Laufe der Evolution gebildet haben und die zudem einen gemeinsamen evolutionären Ursprung haben. So haben beispielsweise alle Primaten ein Herz als ein Organ, das Blut durch den Körper pumpt, obwohl es Unterschiede in den jeweiligen biologischen Strukturen gibt. Nichtsdestotrotz haben sich alle Herzen aus einem gemeinsamen evolutionären Vorfahren entwickelt. Ich möchte nachfolgend bei meinem Beispiel der Furcht bleiben. Eine essentielle Frage ist, was nun beispielsweise allen einzelnen Furchterlebnissen intraindividuell, interindividuell und über verschiedene Spezies hinweg gemeinsam ist. Dieser gemeinsame Faktor rechtfertigt die Zugehörigkeit zum Typus "Furcht" und macht erst möglich, dass alle einzelnen Furchterlebnisse auch tatsächlich Furcht als qualitativen Inhalt haben. Im Sinne der vorliegenden Arbeit muss dieses Gemeinsame ein physikalisches Substrat sein.

Ich schlage vor, einen Typus als die charakterisierende Eigenschaft aller Furchterlebnisse zu verstehen. Das, was alle Furchterlebnisse gemeinsam haben, ist die bestimmte Art und Weise, wie es sich anfühlt, Furcht zu haben. Eine Entität XYZ ist also genau dann ein Furcht-Token, wenn die Entität XYZ die Eigenschaft hat, sich wie Furcht anzufühlen. Gleichzeitig ist es jedoch nicht der Fall, dass der Furcht-Typus selbst sich wie Furcht anfühlt, sondern er wird durch die Eigenschaft, sich wie Furcht anzufühlen, charakterisiert ${ }^{38}$. Hingegen ist ein Token ein bestimmtes Vorkommnis von Furcht, dss Bestimmungen über den Typus hinaus aufweist. Dadurch ist auf der Betrachtungsebene der Neuronenaktivität ein gemeinsamer Faktor festmachbar und eine gewisse Variabilität zugelassen. Aus den Neurowissenschaften wissen wir, dass bei einem Furchterlebnis der zentrale Nucleus der Amygdala als wesentlicher Teil genannt wird, aber auch gleichzeitig weitere Teile des Gehirns, zu denen es strukturelle und funktionale Verbindungen gibt, wie beispielsweise der anteriore zinguläre Gyrus und der präfrontale Kortex. Die konkreten Rollen dieser beteiligten Gehirnstrukturen sind noch nicht

38 Vielleicht ist ein Typus in weiterer Folge ontologisch am besten als ein abstrakter Gegenstand zu verstehen. Das ist eine interessante Frage an sich, dessen Beantwortung ich hier offen lasse. 
eindeutig geklärt, doch für das Ziel, das ich hier verfolge, kann die Beantwortung dieser Frage getrost aufgeschoben werden. Ob sich nun die neuronale Struktur N1, N2, oder Ni als Reduktionsbasis anbietet, macht für meine folgende Argumentation keinen Unterschied. Ich werde deshalb nachfolgend den Platzhalter $N$ verwenden. Intraindividuell und interindividuell können zwar auf neuronaler Ebene geringfügige Variabilitäten auftreten $(\mathrm{X} 1 \ldots \mathrm{Xi})$, doch das ist kein Hindernis für eine Typenreduktion des Mentalen auf das Physikalische, weil die Änderungen unwesentlich sind. Die Änderungen sind beispielsweise ein zusätzlicher Gedanke zum Furchterlebnis ("Ich weiß, dass der Hund nicht über den Zaun springen kann") oder weitere assoziierte Erinnerung ("Der Hund des anderen Nachbarn ist noch größer"). Das Wesentliche bleibt jedenfalls gleich, nämlich eine Aktivität in der neuronalen Struktur N, und darauf kommt es an. Über verschiedene Spezies hinweg zeigen sich in der Tierwelt ebenso homologe Strukturen, die bei Furchtreaktionen aktiviert sind. Auch hier sind innerhalb einer Spezies und zwischen den Spezies gewisse Variabilitäten gegeben (X1 ... Xi). Das Wesentliche bleibt jedenfalls auch hier gleich, nämlich eine Aktivität in der neuronalen Struktur N bzw. deren homologe Struktur, und darauf kommt es an.

Der Typen-Physikalist ist nicht verpflichtet, über Individuen, Spezies und Zeitpunkte hinweg ein und denselben Gehirnzustand zu postulieren, heruntergebrochen auf jedes einzelne Neuron. Er muss lediglich zeigen, dass als Teil dieses gesamten Erregungsnetzwerkes zumindest immer eine einzelne Gehirnstruktur aktiviert ist. Diese Gehirnstruktur ist auf TypenEbene notwendig und hinreichend für ein Furchterlebnis. Aber diese Gehirnstruktur ist jedoch auf Token-Ebene nur notwendig für ein Furchterlebnis zu einem bestimmten Zeitpunkt in einem bestimmten Individuum einer bestimmten Spezies, weil bestimmte Variabilitäten hinzukommen. Die neuronale Struktur $\mathrm{N}$ in Kombination mit den entsprechenden begleitenden Variabilitäten ist schließlich hinreichend für ein ganz bestimmtes Vorkommnis von Furcht. Durch Anwendung der Kriterien der Einfachheit und Sparsamkeit wird die Korrelation durch eine Identität zwischen dem Mentalen (M) und Physikalischen (P) erklärt:

(Mtypus) Ein $\mathrm{M}_{\text {Typus }}$ ist genau dann identisch mit einem $\mathrm{P}_{\text {Typus, }}$, wenn die zugehörigen Tokens zum $\mathrm{M}_{\text {Typus }}$ durch die Eigenschaft $\mathrm{F}$ charakterisiert werden und die zugehörigen Tokens zum $\mathrm{P}_{\text {Typus }}$ durch ein und dieselbe Eigenschaft $\mathrm{F}$ charakterisiert werden.

(Mtoken) Ein $\mathrm{M}_{\text {Token }}$ ist genau dann identisch mit einem $\mathrm{P}_{\text {Token, }}$ wenn der $\mathrm{M}_{\text {Token }}$ durch die Eigenschaft $F$ charakterisiert wird und der $\left.P_{\text {Token }} i\right)$ durch ein und dieselbe Eigenschaft $F$ und ii) durch weitere Eigenschaften $\mathrm{X} 1$ bis $\mathrm{Xi}$ charakterisiert wird. 
Die Typen-Identität zwischen dem Mentalen und dem Physikalischen wird durch die Reduktion der charakterisierenden Eigenschaft auf diejenige (homologe) neuronale Struktur gesichert, die auch bei jeder Token-Identität zwischen dem Mentalen und dem Physikalischen aktiviert ist. Das sichert einen gleichbleibenden Typus, sodass (Mtypus) angenommen werden kann. Damit ist der Weg für eine Typen-Identitätstheorie frei und das Argument der multiplen Realisierbarkeit verliert an Schlüssigkeit, da seine Kernaussagen keine Gültigkeit mehr haben. Gleichzeitig wird der Variabilität einzelner Vorkommnisse Rechnung getragen, sodass (Mtoken) angenommen werden kann.

\subsection{Der A-priori-Physikalismus}

Wir sind bis jetzt soweit gekommen, dass eine gangbare physikalistische Position eine Identitätstheorie ist, welche mentale Typen mit physikalischen Typen identifiziert. Das Ziel ist eine ontologische Reduktion des Mentalen auf das Physikalische. Eine heiß umkämpfte Frage in der Literatur ist, ob die Identitätsaussage " $\mathrm{M}=\mathrm{P}$ " a priori oder a posteriori gewusst werden kann bzw. M vollständig aus dem gesamten physikalischen Wissen abgeleitet werden kann oder eben nicht. An dieser Stelle muss ein technisches Detail eingefügt werden. Dass die Basis für die Ableitung ausschließlich auf das physikalische Wissen aus den Naturwissenschaften beschränkt ist, ist für die Zwecke des A-priori-Physikalisten nicht hinreichend. Einige Autoren haben erfolgreich argumentiert, dass noch zwei weitere Spezifizierungen notwendig sind. Erstens, es muss ein Stoppzeichen ( $T$ ) eingefügt werden, sodass aus der physikalischen Basis auch negative Existenzaussagen folgen, wie beispielsweise in der Form "Es gibt kein Ektoplasma". Damit geschieht die Ableitungen von $M$ aus (P \& T):

This loophole can be closed by conjoining to $P$ a "that's all" statement $T$, asserting that our world is a minimal world satisfying $P$. Intuitively, this statement says that our world contains what is implied by $P$, and only what is implied by $P$. (Chalmers \& Jackson, 2001, S. 317)

$T$ is a single totality sentence, saying that our world is a minimal scenario satisfying $P \ldots T$ says in effect 'that's all': the world is no bigger than it needs to be to accommodate the truth of $P[\ldots]$. (Chalmers, 2012, S. 111)

Zweitens, es müssen bestimmte indexikalische Ausdrücke (I) augenommen werden, um beispielsweise den folgenden Satz "Ich habe jetzt den Zucker aus meinem Einkaufswagen verstreut"39. Damit geschieht die Ableitungen von M aus (P \& T \& I):

39 Dieses Beispiel ist an Perry (1979) angelehnt. 
This loophole can be closed by conjoining to PT some locating information (or indexical information) I. I can be thught of as a "you are here" marker added to the objective map given by PT. I can consist of the conjunction of any two truth 'I am $A$ ' and 'now is $B$ ', where $A$ is an identifying description of myself (or the subject in question) and $B$ is an identifying description of the current time.

(Chalmers \& Jackson, 2001, S. 318)

I includes at least two indexical truths. We can suppose that we have picked out a subject $s$ and time $t$ relative to which we are assessing scrutability...

Then I will include truths of the form 'I am $D_{1}$ ' and 'Now is $D_{2}{ }^{\prime} \ldots$ These $\left[D_{1}\right.$ and

$D_{2}$ ] will typically be identifying descriptions, picking out $s$ and $t$ uniquely [...]. (Chalmers, 2012, S. 111)

Ich werde nachfolgend immer ( $\mathrm{P} \& \mathrm{~T} \& \mathrm{I})$ als Ableitungsbasis meinen, der Einfachheit halber jedoch immer ausschließlich $P$ verwenden.

In der Diskussion zwischen dem A-priori-Physikalisten und dem A-posterioriPhysikalisten wird vor allem ein genaueres Verständnis der Begriffe "a priori" und "a posteriori" notwendig 40 :

(A priori) Eine Aussage $p$ ist genau dann a priori wahr oder falsch, wenn für die Feststellung des Wahrheitswertes von $p$ nicht auf Erfahrung zurückgegriffen werden muss.

(A posteriori) Eine Aussage $p$ ist genau dann a posteriori wahr oder falsch, wenn für die Feststellung des Wahrheitswertes von $p$ auf Erfahrung zurückgegriffen werden muss.

Der A-priori-Physikalist glaubt daran, dass das Verhältnis zwischen dem Mentalen und dem Physikalischen a priori ist und er beruft sich auf analoge Beispiele wie das einer geometrischen Figur. Sobald drei Punkte zu einem bestimmten Winkel zueinander stehen, lässt sich daraus die Figur eines rechtwinkeligen Dreieckes ableiten. Alles, was es braucht, ist die Anordnung der drei Punkte in bestimmter Art und Weise, und das Dreieck kommt ohne weiteres Zutun und notwendigerweise hinzu. Es ist nicht notwendig, einen Blick in die Welt zu werfen und

\footnotetext{
40 Ich folge in den Definitionen im Großen und Ganzen Chalmers (2012), der in seinem siebten Exkurs beide Begrifflichkeiten umfassend einführt: "I have said that a sentence $S$ is a priori for a subject $s$ if $s$ is in a position to know $S$ with justification independent of experience" (S. 185) bzw. "A subject knows a posteriori (or equivalently, knows empirically) that $p$ when she knows that $p$ with justification that depends on experience" (S. 185-186).
} 
Erfahrungen im Zeichnen von Dreiecken zu gewinnen. Durch die Reduktion von M auf P bzw. die Ableitbarkeit von $\mathrm{M}$ aus $\mathrm{P}$ würde somit alles Mentale zu etwas Physikalischem machen.

Der A-posteriori-Physikalist hingegen glaubt daran, dass das Verhältnis zwischen dem Mentalen und dem Physikalischen a posteriori ist und er beruft sich auf Kripke (1980) und seiner Arbeit zu notwendigen Aussagen a posteriori und folgen seiner Analyse, dass "Wasser $=\mathrm{H} 2 \mathrm{O}$ ", "Hesperus = Phosphorus" oder "Superman = Clark Kent" notwendig und a posteriori sind. Um diese Identitätsaussagen auch verstehen zu können, muss man einen Blick in die Welt werfen, denn nur so kann man beispielsweise erfahren, dass der Morgenstern (Phosphorus) und der Abendstern (Hesperus) eigentlich ein und derselbe Planet sind. Die Ausdrücke, die das Identitätszeichen flankieren, beziehen sich in jeder möglichen Welt auf denselben Referenten und sind nach Kripke starre Bezeichnungsausdrücke (rigid designators). Auch hier wird somit durch die Reduktion von $\mathrm{M}$ auf $\mathrm{P}$ bzw. die Ableitbarkeit von $\mathrm{M}$ aus $\mathrm{P}$ alles Mentale zu etwas Physikalischem und der physikalische Begriff und der mentale Begriff referieren beide auf dasselbe Existierende, nämlich auf etwas Physikalisches.

Ich möchte nachfolgend zeigen, dass der A-priori-Physikalismus den Vergleich mit dem A-posteriori-Physikalismus gewinnt. Hierfür bringe ich vier Argumente von Chalmers und Jackson (2001), Jackson (2007), Gole (2008a) und Chalmers (2012), die am überzeugendsten die wesentlichen Vorteile des A-priori-Physikalismus hervorkehren.

Chalmers und Jackson (2001) ${ }^{41}$ argumentieren, erstens, dass der A-posteriori-Physikalist primitive Identitäten zwischen $\mathrm{M}$ und $\mathrm{P}$ abduktiv postulieren muss. Sie sind deshalb primitiv, weil die postulierte Identität auf nichts anderes zurückführbar ist, und die Feststellung der Identität durch einen Schluss auf die beste Erklärung geschieht. Hingegen leitet der A-priori-Physikalist die Identität zwischen $\mathrm{M}$ und $\mathrm{P}$ deduktiv aus $\mathrm{P}$ ab, somit ist die Identität nicht primitiv, sondern auf $P$ zurückführbar. Unsere Kriterien wissenschaftlicher Theorien anwendend ist der A-prioriPhysikalismus auch deshalb ontologisch sparsamer, weil er keine primitiven Identitäten postuliert. Darüber hinaus ist er logisch stärker, weil deduktiv auf die Identität geschlossen wird und somit kein abduktives Schlussfolgern mehr notwendig ist. Zweitens, falls es überhaupt primitive Identitäten gibt, dann sind diese auch nicht epistemisch transparent. Ein Sachverhalt ist für Chalmers und Jackson genau dann epistemisch transparent, wenn es dafür eine Erklärung gibt. Im Falle einer primitiven Identität zwischen $M$ und $P$ gibt es jedoch keine ausreichende Erklärung. Wenn sich die Identität jedoch zwischen $\mathrm{M}$ und $\mathrm{P}$ jedoch a priori aus $\mathrm{P}$ ableiten lässt, dann ist das eine Erklärung, warum sie vorherrscht. Aus diesem Grunde ist eine A-priori-Identität epistemisch transparent, jedoch keine A-posteriori-Identität. Bei Anwendung unserer Kriterien wissenschaftlicher Theorien ist der A-priori-Physikalismus somit auch einfacher als der A-posteriori-Physikalismus.

\footnotetext{
${ }^{41}$ Dieser Artikel ist als Reaktion auf Block und Stalnaker (1999) zu sehen, die für den A-posteriori-Physikalismus argumentieren.
} 
Jacksons (2007) ${ }^{42}$ Argument für den A-priori-Physikalismus bezieht sich auf die Semantik der Identitätsaussagen. Im Allgemeinen geht Jackson davon aus, dass kompetente Sprachbenützer wissen, was bestimmte Äußerungen in einer bestimmten Sprache bedeuten (S. 190):

Psychological terms and the sentences employing them represent things as being a certain way, and we know what that way is .... The alternative is to hold that we don't know what we are saying when we say that someone is in pain.

Jackson nimmt diese Überlegungen als Ausgangspunkt für sein folgendes Argument (S. 190):

The instantiation of what is being ascribed is what makes the ascription true but, for physicalists, the only properties instantiated are physical ones ... On pain of making all psychological ascriptions false, physicalists must allow that their very understanding of psychological predicates delivers to them the physical properties ascribed by those predicates ... [This] tells us how things have to be if that sentence [e.g., "someone is in pain"] is to be true, but that "how things have to be" had better be physical if physicalism is to be true. But then the passage from the physical to descriptions of how things are in psychological terms is accessible from understanding alone. That's tantamount to priori physicalism.

Nach Jackson haben Sätze wie "Markus hat Schmerzen" für kompetente Beherrscher der deutschen Sprache eine Bedeutung, nämlich die, dass Markus Schmerzen hat. Wenn es tatsächlich der Fall ist, dass Markus Schmerzen hat, dann ist es auch der Fall, dass unter der Gültigkeit des Physikalismus die Eigenschaft "Schmerzen zu haben" nichts anderes als eine physikalische Eigenschaft in der Form "eine neuronale Aktivität $\mathrm{N}$ zu haben" identisch ist. $\mathrm{Ob}$ man nun ein Anhänger des A-priori-Physikalismus oder des A-posteriori-Physikalismus ist, beide Lager stimmen damit überein, dass Schmerzen mit der Aktivierung einer bestimmten neuronalen Struktur identisch sind. Durch diese bereits zugebilligte Identität zwischen dem Mentalen und dem Physikalischen führt das Sprechen über Schmerzen zur entsprechenden neuronalen Struktur. Weil nach dieser Analyse das Mentale zum Physikalischen führt, führt als weitere Konsequenz daraus auch das Physikalische zum Mentalen. Die Ableitung des Mentalen aus dem Physikalischen ist damit ohne Rückgriff auf Erfahrung möglich. Und das ist nichts anderes als zu sagen, dass $\mathrm{M}$ aus $\mathrm{P}$ a priori abgeleitet werden kann.

42 McLaughlin (2007) verteidigte in seinem Aufsatz den A-posteriori-Physikalismus und wurde im selben Sammelband wie auch Jacksons Artikel abgedruckt. 
Gole (2008a) bezieht sich auf die Forderung von A-posteriori-Physikalisten, dass eine Person, die ein subjektives Erlebnis wie Schmerzen, Furcht oder Liebe erfährt, eine besondere Art eines Begriffes lernt. Es handelt sich um einen phänomenalen Begriff, der sich auf das entsprechende Quale bezieht und nur durch Erlebnisse erworben werden kann. Stoljar (2005) hat dafür den Ausdruck "Strategie der phänomenalen Begriffe" geprägt und Anhänger dieser Strategie vertreten die Ansicht, dass phänomenale Begriffe unabhängig von physikalischen Begriffen sind, aber gleichzeitig Teil einer physikalischen Beschreibung der Welt sind. Sie sind deshalb unabhängig von physikalischen Begriffen, weil sie eine Begriffsgattung sui generis sind und nur direkt und unmittelbar durch ein subjektives Erlebnis selbst gebildet werden können. Trotz alledem sind sie Teil einer physikalischen Beschreibung der Welt, weil mit phänomenalen Begriffen etwas Physikalisches beschrieben wird, denn letztendlich sind auch Qualia für jeden Physikalisten nichts anderes als etwas Physikalisches. Phänomenale Begriffe sind beispielsweise demonstrative Begriffe (z.B. Loar, 1997) ${ }^{43}$ mit der Form "diese Art von Erlebnis" und beziehen sich dadurch auf das entsprechende Quale. Unabhängig von dieser genaueren Spezifizierung hat sich das ursprüngliche Bewusstseinsproblem lediglich verschoben: Denn nun wird nicht mehr gefragt "Wie passen Qualia in unsere physikalistische Welt?", sondern es wird gefragt "Wie passen phänomenale Begriffe in unsere physikalistische Welt?". Dieses neue Problem entsteht durch den Sonderstatus von phänomenalen Begriffen, die einerseits unabhängig von physikalischen Begriffen sind und andererseits dennoch etwas Physikalisches als einen Referenten haben. Das Einführen von phänomenalen Begriffen und die darauffolgende Verlagerung des ursprünglichen Problems hat auch Konsequenzen bei Anwendung unserer beiden Kriterien wissenschaftlicher Theorien, denn es geht entgegen einer Einfachheit, weil dadurch nichts Wesentliches zur Lösung des Problems beigetragen wurde. Gleichzeitig spricht die Einführung einer neuen, fundamentalen Begriffsgattung auch gegen eine ontologische und begriffliche Sparsamkeit.

Schließlich bringt Chalmers (2012) ein positives Argument für eine A-priori-Ableitbarkeit mit seinem "Aufladungsargument" (frontloading argument). Falls die Ableitung von M aus P (d.h. $P \rightarrow M)$ aus irgendwelchen Gründen durch Subjekt $S$ noch nicht a priori möglich ist und $S$ noch eine weitere empirische Evidenz $\mathrm{E}$ benötigt, dann kann $\mathrm{E}$ getrost in das Antezedenz aufgenommen, sodass gilt: $(P \& E) \rightarrow M$. S kann somit jetzt $M$ aus $P \& E$ a priori ableiten. Das Hinzufügen weiterer empirischer Evidenzen kann immer weiter wiederholt werden und das Antezedenz weiter "aufgeladen" werden, sodass gilt: (P \& E1 \& .. \& Ei) $\rightarrow$ M. Egal, welche Beobachtung oder welche Erfahrung man noch braucht, man packt es in das Antezedenz hinein. Mit diesem Aufladungsargument wird es intuitiv einleuchtender, wie eine A-priori-Ableitbarkeit tatsächlich geschehen kann und dass das Antezedenz immer größer werden kann. Dies spricht

${ }^{43}$ Es finden sich auch viele weitere Versionen, wie phänomenale Begriffe weiter beschrieben werden können. Beliebte Versionen verstehen darunter indexikalische Begriffe (z.B. Perry, 2001), anführende Begriffe (z.B. Papineau, 2002) oder auch wahrnehmende Begriffe (z.B. Papineau, 2007). Ein generalisiertes Argument gegen alle Versionen dieser Strategie der phänomenalen Begriffe ist insbesondere in Chalmers (2007) zu finden. 
auch für die Entwicklung der Naturwissenschaften, welche die einzelnen Erkenntnisse in den physikalistischen Wissenskanon einbringen, sodass P im Prinzip eine große Konjunktion all unseres physikalistischen Wissens darstellt.

\subsection{Der Physikalismus als Sprachprogramm}

Ich habe bis jetzt argumentiert, dass eine Identitätstheorie mentale Typen auf physikalische Typen reduziert, indem diese Identität der beiden Typen a priori aus dem gesamten physikalischen Wissen abgeleitet werden kann. Um nun bestimmen zu können, ob der Physikalismus und die Identitätsaussage "M = P" wahr oder falsch sind, gibt es im Großen und Ganzen zwei Möglichkeiten. Einerseits kann der Physikalismus analytisch wahr sein oder synthetisch wahr sein:

(Analytisch) Eine Aussage $p$ ist genau dann analytisch wahr oder falsch, wenn für die Feststellung des Wahrheitswertes von $p$ die Bedeutung der involvierten Wörter ausreicht ${ }^{44}$.

(Synthetisch) Eine Aussage $p$ ist genau dann synthetisch wahr oder falsch, wenn für die Feststellung des Wahrheitswertes von $p$ die Bedeutung der involvierten nicht Wörter ausreicht.

Unter einem analytisch wahren Satz fällt beispielsweise die Aussage "Junggeselle = unverheirateter Mann". Um zu wissen, ob beides dasselbe ist, muss ich lediglich die Bedeutung beider Begriffe kennen, weil der Ausdruck "Junggeselle" als ein unverheirateter Mann definiert wird. Diese Aussage ist gleichzeitig auch a priori, denn es kann ohne Rückgriff auf Erfahrung gewusst werden. Hingegen sind synthetisch wahre Sachverhalte beispielsweise Aussagen wie "Die Geburtenrate korreliert mit dem Storchenflug". Hier ist nicht aufgrund der Bedeutung der Begriffe klar, ob die Korrelation zwischen der Geburtenrate und dem Storchenflug besteht, weil es mehr braucht, um den Wahrheitswert zu bestimmen. Ob nun diese Korrelation besteht, ist gleichzeitig auch eine Frage a posteriori, weil es ein empirischer Sachverhalt ist. Aussagen a posteriori sind auch immer gleichzeitig synthetische Aussagen, doch der Umkehrschluss ist nicht richtig. Synthetische Aussagen können nicht nur a posteriori sein, sondern Kant (1787/1929) folgend auch a priori sein. So werden Aussagen wie beispielsweise " $2+2=4$ " in einer

\footnotetext{
${ }^{44}$ Um zu entscheiden, ob eine Aussage analytisch wahr ist, ist manchmal die Bedeutung eines ganzen Satzes ausschlaggebend und nicht ausschließlich die Bedeutung der involvierten Wörter alleine. So kann beispielsweise die Bedeutung eines Satzes durch das Setzen eines Beistriches gravierende Änderungen erfahren. Womöglich entscheidet ein Beistrich zwischen Leben und Tod: "Ich komme, nicht erschießen!" und "Ich komme nicht, erschießen!". Der Einfachheit halber werde ich mich für die restliche Arbeit auf das verkürzte Verständnis des Analytischen beschränken, das nur die Bedeutung der involvierten Wörter heranzieht und den Satzaufbau vernachlässigt.
} 
Kant'schen Tradition als synthetisch und a priori angesehen, denn ich benötige zur

Wahrheitswertbestimmung mehr als die Bedeutung der involvierten Ausdrücke, obwohl ich diese mathematische Aussage ohne Rückgriff auf Erfahrung weiß.

Gegen eine tragfähige Unterscheidung zwischen den Begriffen "analytisch" einerseits und "synthetisch" andererseits hat sich als erster Quine (1951) gerichtet. Dieses Dogma einer hinreichend klaren Unterscheidung greift er damit an, indem "Analytizität" gewöhnlicherweise auf vier verschiedene Arten verstanden werden kann. Es wird entweder im Rahmen von expliziten Definitionen verstanden, bei dem ein Ausdruck auf einen anderen Ausdruck reduziert wird, oder im Rahmen einer Synonymie, bei der beide Ausdrücke in allen möglichen Situationen salva veritate ausgetauscht werden können, oder im Rahmen von bestimmten semantischen Regeln oder im Rahmen der Verifikationstheorie der Bedeutung. Diese Begriffe, in dessen Rahmen Analytizität verstanden wird, setzt nach Quine wiederum ein bereits vorhandenes Verständnis von Analytizität voraus. Aus diesem Grunde ist Analytizität auch gleichzeitig zirkulär und sollte deshalb als Begriffskategorie überhaupt abgelehnt werden.

Um auf Quines Vorwurf der Zirkularität zu antworten, könnte man beispielsweise sagen, dass der Begriff der Analytizität zwar zirkulär ist, dieser Zirkel aber kein vitiöser Zirkel ist. Es handelt sich vielmehr um einen hermeneutischen Zirkel, bei dem die Zirkularität notwendig ist, um den Begriff der Analytizität erst richtig zu verstehen. Eine andere Möglichkeit wäre, Chalmers' Argumentation zu folgen und zu zeigen, dass solch ein Zirkel eigentlich bei einer Vielzahl an philosophischen Schlüsselbegriffen auftritt, wie beispielsweise bei Bewusstsein, Verursachung, Freiheit, Wert und Existenz (Chalmers, 2011, vgl. S. 388). Schließlich könnte Quine auch provokativer entgegnet werden, nämlich mit der Frage, warum Analytizität nicht auch als ein fundamentaler Begriff aufgefasst werden sollte? Vielleicht ist es schlichtweg ein grundlegendes Faktum, dass einige Sätze analytisch sind und andere wiederum synthetisch. Möglicherweise kann eine zufriedenstellende Replik an Quine gegeben werden, sodass eine tragbare Unterscheidung zwischen dem Analytischen und dem Synthetischen vorgebracht werden kann. Ich bin zuversichtlich, dass es solch eine Replik gibt, sei es eine der vorher erwähnten Antworten oder eine beliebige andere Antwort. Ich werde keine solche kugelsichere Replik anbieten, sondern nehme für die weitere Diskussion eine robuste Unterscheidung zwischen dem Analytischen und dem Synthetischen als Ausgangspunkt an.

\subsubsection{Das Übersetzungsargument}

Den Physikalismus betreffend ist die Frage zentral, ob nun ein mentaler und ein physikalischer Begriff nicht nur bei Wahrheit des Physikalismus koreferentiell sind, sich also auf denselben Referenten beziehen, sondern ob sie auch synonym sind. Beide Begriffe beziehen sich auf denselben Referenten, weil auf ontologischer Ebene die Reduktion von beispielsweise Furcht auf eine neuronale Aktivität geschehen ist und sich deshalb der mentale Begriff "Furcht" und der 
physikalische Begriff "N-Aktivität" beide auf die entsprechenden physikalischen Vorgänge in der neuronalen Struktur $\mathrm{N}$ beziehen. Ob sie nun auch synonym sind und in jeglichem Kontext wahrheitswerterhaltend ausgetauscht werden können, ist eine andere Frage. Bei vorhandener Synonymität würde auch der mentale Begriff auf den physikalischen Begriff reduziert werden können und wir hätten denselben analytischen Status der Aussage "Furcht = N-Aktivität" wie bei "Junggeselle = unverheirateter Mann", weil "Furcht" in einem physikalischen Vokabular einer NAktivität definiert werden könnte.

Ob eine ontologische Reduktion von $\mathrm{M}$ auf $\mathrm{P}$ auch eine begriffliche Reduktion bedeutet, wird innerhalb der gegenwärtigen Philosophie des Geistes typischerweise verneint. So schreiben beispielsweise Chalmers und Jackson (2001, S. 321):

It is sometimes claimed that for $A \rightarrow B$ to be a priori, the terms in $B$ must be definable using the terms of $A$. On this view, a priori entailment requires definitions, or explicit conceptual analyses: that is, finite expressions in the relevant language that are a priori equivalent to the original terms, yielding counterexample-free analyses of those terms. This is not our view.

Smart, ein Vertreter der Identitätsthese der ersten Stunde, geht sogar soweit, zu sagen, dass eine Übersetzbarkeit mentaler Ausdrücke in physikalische Ausdrücke absurd ist und der Physikalist solch eine absurde These nicht vertreten muss (Smart, 2007, dritter Absatz seines Enzyklopädie-Eintrages):

In taking the identity theory (in its various forms) as a species of physicalism, I should say that this is an ontological, not a translational physicalism. It would be absurd to try to translate sentences containing the word 'brain' or the word 'sensation' into sentences about electrons, protons and so on. Nor can we so translate sentences containing the word 'tree'. After all 'tree' is largely learned ostensively, and is not even part of botanical classification. If we were small enough a dandelion might count as a tree. Nevertheless a physicalist could say that trees are complicated physical mechanisms.

Aus diesen beiden Textpassagen kommt heraus, dass die Ableitbarkeit des Mentalen vom Physikalischen unabhängig von einer expliziten Definition des Mentalen in physikalische Begriffe ist. Die Ableitbarkeit ist keine begriffliche Ableitbarkeit, es ist vielmehr eine Ableitbarkeit in der Form, dass wenn immer auch $P$ gegeben ist, $M$ notwendigerweise logisch folgt. Bei Gültigkeit der Identitätsthese folgt, dass die Identität zwischen beiden Typen auf ontologischer Ebene gilt: $M=P$. 
Doch eine begriffliche Reduktion von $\mathrm{M}$ auf $\mathrm{P}$ wurde ursprünglich in der Formulierung eines semantischen Physikalismus vertreten. Der Ursprung eines semantischen Physikalismus ist im logischen Positivismus zu finden und Anhänger des Positivismus waren Kritiker der Metaphysik und bezeichneten metaphysische Fragen als Scheinprobleme. Zwei der berühmtesten Anhänger des Positivismus und Mitglieder des Wiener Kreises waren Rudolf Carnap und Otto Neurath und hatten großen Einfluss auf Carl Hempel. Alle drei sprachen von einem Physikalismus, doch für sie war klar, dass es sich eigentlich um keine metaphysische These handelt. Der Physikalismus war die Art und Weise, wie in den Wissenschaften mit einer universellen Sprache gesprochen werden soll (Carnap, 1931; Neurath, 1931; Hempel, 1935). In diesem Zusammenhang behauptete Carnap sogar, dass jeder bedeutungsvolle Ausdruck oder Satz, auch innerhalb der Domäne der Psychologie, in die physikalische Sprache ohne Verlust übersetzt werden könne. Eine markante Textstelle ist in Carnap (1932, S. 142) zu finden:

Sogenannte psychologische Sätze, mögen sie nun konkrete Sätze über Fremdpsychisches, über vergangenes Eigenpsychisches oder über gegenwärtiges Eigenpsychisches [...] sein, sind stets in die physikalische Sprache übersetzbar. Und zwar bezieht jeder psychologische Satz sich auf physikalische Vorgänge am Leib der betreffenden Person(en). Daher ist die Psychologie ein Teilgebiet der Einheitswissenschaft auf physikalischer Basis. [...] Dann können wir unsere These, eine Teilthese des Physikalismus, so fassen: die Psychologie ist ein Zweig der Physik.

Ähnlich schreibt Hempel (1935, S. 18)

The result of the preceding considerations can now be summed up as follows: All psychological statements [...] are translatable into statements which do not involve psychological concepts, but only the concepts of physics. The statements of psychology are consequently physicalistic statements. Psychology is an integral part of physics.

Obwohl Carnap, Neurath und Hempel unter einem Physikalismus keine metaphysische These verstanden, lässt sich ihr Ansatz leicht auf die gegenwärtige Diskussion übertragen. Folgt man Carnaps Ansatz, dann lässt sich das Mentale vom Physikalischen eben aufgrund der expliziten Definition des Mentalen in physikalische Begriffe ableiten. Bei Gültigkeit der Identitätsthese folgt, dass die Identität zwischen beiden Typen nicht nur auf ontologischer Ebene gilt, sondern dass auch eine Identität im Sinne einer Synonymität zwischen mentalen und physikalischen

45 Die Seitenanzahl bezieht sich auf den Sammelband von N. Block (1980). 
Begriffen gilt. Was auch immer eine finale physikalische Theorie für uns bereithält, alle mentalen Begriffe lassen sich in physikalische Begriffe übersetzen.

Es bleibt die Antwort auf die Frage offen, welchem Ansatz man nun folgen soll. Gegenwärtige Philosophen des Geistes sind abgeneigt, eine begriffliche Reduktion und eine Synonymität zwischen unserem mentalen Vokabular und dem physikalischen Vokabular anzunehmen. Doch kann man gute Gründe für eine begriffliche Reduktion finden? Ich denke, solch ein Argument gibt es, und ich werde es nachfolgend vorstellen. Ich möchte zum Zwecke des Arguments annehmen, dass keine Synonymität zwischen einem mentalen und physikalischen Begriff bei Ableitung der Identität " $\mathrm{M}=\mathrm{P}$ " gegeben ist und die Ableitung keine begrifflich-definitorische Implikation ist. Die Ableitbarkeit ist vielmehr in dem Sinne zu sehen, dass notwendigerweise gilt, wenn $P$ gegebenen ist, auch die Identität zwischen $M$ und $P$ folgt. Damit geschieht keine Wortmagie am Schreibtisch, welche eine Synonymität einfach behauptet. Überhaupt keine Wortmagie wäre es jedoch, wenn eine begriffliche Identität deduktiv aus einem Argument folgt. Genau solch ein Argument möchte ich bringen, das zeigt, dass eine begriffliche Reduktion aus einer ontologischen Reduktion folgt. Ich nenne es Übersetzungsargument (siehe Vorgängerüberlegungen in Gole, 2008a):

(1) Furchterlebnis = Aktivierung der neuronalen Struktur N.

(2) Jede Aktivierung der neuronalen Struktur $\mathrm{N}$ ist ausschließlich in physikalischen Begriffen definierbar.

(3) Also ist jedes Furchterlebnis ausschließlich in physikalischen Begriffen definierbar.

Wenn wir annehmen, dass der A-priori-Physikalismus wahr ist, physikalische Basisentitäten oder davon abgeleitete Entitäten die Reduktionsbasis darstellen und eine ontologische Reduktion von $\mathrm{M}$ auf $\mathrm{P}$ gelungen ist, dann ist auch jedes Furchterlebnis als Typus mit einer Aktivierung in der neuronalen Struktur $\mathrm{N}$ als Typus identisch. Das ist genau das, was Prämisse (1) aussagt. Dieser Sachverhalt ist unproblematisch für Anhänger der Identitätsthese und stellt sozusagen deren Grundaussage dar. Nehmen wir weiter an, dass jede Aktivierung der neuronalen Struktur N zur Gänze durch physikalische Begriffe im weitesten Sinne definiert werden kann. Aufgrund des Umfangs einer finalen physikalischen Theorie lassen sich alle physikalischen Gegebenheiten in physikalischer Sprache beschreiben und definieren. Prämisse (2) sagt genau das. Auch dieser Punkt ist unproblematisch für Anhänger der Identitätsthese und sogar unproblematisch für Dualisten.

Aus diesen beiden Prämissen folgt schließlich die Konklusion (3): Auch jedes Furchterlebnis kann zur Gänze durch physikalische Begriffe definiert werden. Der Ausdruck "Aktivierung der neuronalen Struktur N" aus (2) kann nun durch "Furchterlebnis" aufgrund deren Identität aus (1) ausgetauscht werden. Die Akzeptanz dieser Konklusion hat auch zur Folge, dass der Physikalismus nun auch begrifflich sparsamer als (fast) alle anderen physikalischen 
Bewusstseinstheorien wird, weil nun eine Art von Begriffen auf eine andere Art von Begriffen reduziert wurde. So wie es nicht mehr nötig ist, den Begriff "unverheirateter Mann" zu verwenden, falls über Junggesellen gesprochen wird, so ist es auch nicht mehr nötig, über Qualia in mentalen Begriffen zu sprechen, weil das entsprechende synonyme physikalische Vokabular zur Verfügung steht. Es ist kein Bedeutungsverlust passiert, sondern lediglich ein Verlust an begrifflicher Pluralität, das wiederum positiv zu werten ist im Lichte des Kriteriums der begrifflichen Sparsamkeit.

\subsubsection{Einwände und Repliken zum Übersetzungsargument}

Philosophie wäre nicht Philosophie, wenn es nicht auch gegenteilige Überzeugungen gäbe. Ich möchte zwei Einwände gegen eine Synonymität zwischen mentalen und physikalischen Begriffen vorbringen, die sich oftmals in dem einen oder anderen Gewand in der Diskussion finden. Als erster Einwand wird gebracht, dass eine Ableitbarkeit des Mentalen aus dem Physikalischen und schließlich die Feststellung deren Identität nicht auch gleichzeitig eine Definierbarkeit des Mentalen in physikalischen Begriffen bedeutet. So kommt man beispielsweise von der Aussage "Es gibt einen roten Ball" zur abgeleiteten Aussage "Es gibt einen färbigen Ball", ohne dass der Ausdruck "färbig" weder durch den Ausdruck "rot" noch durch andere involvierte Wörter definiert wird (Chalmers, 2012, vgl. S. 14).

Als Replik deute ich einen offensichtlichen Unterschied zwischen dem Übersetzungsargument und der Aussage, dass "färbig" nicht durch "rot" definiert wird. Der Unterschied besteht darin, dass zwischen den Eigenschaften "färbig zu sein" und "rot zu sein" keine Identität besteht wie zwischen den Eigenschaften "ein Furchterlebnis zu haben" und "eine Aktivierung in der neuronalen Struktur $\mathrm{N}$ zu haben". Streng genommen verfehlt der Einwand von Chalmers das Übersetzungsargument als Ziel, weil keine hinreichende Parallelität zwischen beiden Argumentationsstrukturen gegeben ist, da beim Übersetzungsargument eine Identität in Prämisse (1) im Spiel ist, bei Chalmers diese jedoch fehlt.

Als zweiter Einwand wird gebracht, dass, falls die ontologische Reduktion von $\mathrm{M}$ auf $\mathrm{P}$ und die begriffliche Reduktion von M-Begriffen auf P-Begriffen aufgrund deren Synonymie angenommen wird, dies den Physikalismus auch analytisch wahr macht. Der Physikalismus ist so eine Wahrheit, die notwendigerweise gilt und die nicht falsifiziert werden kann. Hier wird eine scheinbar absurde Konsequenz des Übersetzungsarguments aufgezeigt, falls die Konklusion akzeptiert wird.

Als Replik stelle ich zuerst fest, dass die Relation zwischen M und P die der Identität ist. Diese Ableitung der Identität zwischen $M$ und $P$ aus all unserem physikalischen Wissen ist a priori und ebenso analytisch, denn ich weiß ohne Rückgriff auf Erfahrung, dass $M$ und $P$ identisch sind, und ich kenne dessen Wahrheitswert aufgrund der Bedeutung der involvierten Wörter. Trotzdem ist These des Physikalismus eine kontingente Wahrheit, die nicht 
notwendigerweise in allen möglichen Welten gilt und somit auch in unserer aktualen Welt falsch sein kann. Konkret ist es das physikalische Wissen, das kontingent ist, und das schließlich als Basis für die A-priori-Ableitbarkeit der Identität zwischen M und P sowie der Synonymität zwischen den mentalen Begriffen und den physikalischen Begriffen fungiert. Hier ist auch wichtig zu betonen, dass dabei ebenso die Identitätsaussage kontingent ist, weil das Physikalische davon abhängt, wie unsere aktuale Welt beschaffen ist. Gleichzeitig gibt es auch einen Teil im Physikalismus, der notwendig ist: Falls all unsere physikalischen Erkenntnisse sich in einer bestimmten Art und Weise darstellen, dann folgt die Identität von $\mathrm{M}$ und $\mathrm{P}$ und auf Basis des Übersetzungsarguments a fortiori ist alles Mentale ausschließlich in physikalische Begriffe definierbar. Der Physikalismus verlangt eine Notwendigkeit de dicto in der folgenden Form:

(de dicto) Es gilt notwendigerweise: Wenn $\mathrm{P}$, dann $\mathrm{M}=\mathrm{P}$.

Hingegen kann der Physikalismus falsch sein, wenn sich all unsere physikalischen Erkenntnisse in einer anderen Art und Weise entwickeln, sodass nicht mehr die Identität zwischen dem Mentalen und dem Physikalischen abgeleitet werden kann, weil unsere Ontologie durch fundamental mentale Entitäten ergänzt werden muss. Ähnlich schreibt Stoljar (2010, S. 130-131) zur Kontingenz des Physikalismus:

For example, consider the statement 'my aunt's favorite property necessitates my uncle's favorite property'. This statement is a necessitation statement since the relation it asserts to obtain is necessitation. But is nevertheless contingent, since it depends for its truth on the preferences of my aunt and uncle. If my aunt likes being red best, and my uncle likes being colored best, the statement is true; alternatively if my aunt likes being red best and my uncle likes being square, it is false... Once we accept the point that statements of necessitation in general can be contingent, it is an easy matter to see how physicalism in particular can be. Physicalism is the thesis that every instantiated property is necessitated by some physical property. This is a necessitation statement, but it is also contingent because the extension of the expressions 'every instantiated property' and 'some physical property' differs from world to world.

In dieselbe Richtung geht Jacksons Ansatz, der die Kontingenz der Identitätsaussage " $\mathrm{M}=\mathrm{P}$ " so versteht (Jackson, 2012, S. 159):

The last question on notice was the coherence of the notion of contingent identity. In one sense we have just answered this question. What the identity theorist is saying is that brain state kinds get to be the mental state kinds that 
they are because of what they do, and that what they do is a contingent property of them, the brain states.

Diese Analyse zeigt uns, dass ein Physikalismus a priori und analytisch wahr sein kann, aber trotzdem auch kontingent sein kann. Der springende Punkt dabei ist, wie unsere Welt konkret beschaffen ist: Die komplette fundamentale Aufmachung unserer Welt hätte anders sein können. Das ist der kontingente Teil des Physikalismus, der die These des Physikalismus insgesamt kontingent macht. Alle Korrelationen zwischen Erlebnissen und Gehirnprozessen, alle neuen Erweiterungen der Elementarteilchen und dergleichen sind genau so, wie sie sind, weil unsere Welt genau so ist, wie sie ist. Unsere Welt hätte auch anders sein können, sodass die Elementarteilchen aus geistiger Substanz wären. In solch einer Welt wäre der Physikalismus falsch. Während der Physikalismus in unserer aktualen Welt wahr ist, gibt es mögliche Welten, komplett unähnlich zu unserer aktualen Welt, in denen der Physikalismus falsch ist.

Als dritter Einwand könnte vorgebracht werden, dass ich einen intensionalen Fehlschluss begehe, weil etwas zu definieren einen intensionalen Kontext darstellt, vergleichbar damit, wenn unter bestimmten Umständen an etwas geglaubt wird. Damit ist der Schluss der beiden Prämissen auf die Konklusion des Übersetzungsarguments nicht gerechtfertigt. Betrachten wir die folgenden Zeilen:

(1') Markus glaubt, dass Richard Bachman der Autor von "Der Fluch" ist.

(2') Markus glaubt nicht, dass Stephen King der Autor von "Der Fluch" ist.

In diesem Fall kann rationalerweise geglaubt werden, dass Richard Bachman der Autor des besagten Buches ist, und gleichzeitig rationalerweise geglaubt werden, dass Stephen King nicht der Autor desselben Buches ist, obwohl Richard Bachmann und Stephen King ein und dieselbe Person sind. Auch wenn sogar die Identität zwischen beiden Personen bekannt ist, dann kann Markus (1') und gleichzeitig (2') für wahr halten. Die Ausdrücke "Richard Bachmann" und "Stephen King" sind in diesem Kontext nicht wahrheitswerterhaltend, salva veritate, miteinander austauschbar.

Meine Replik zielt darauf ab, dass kein analoger intensionaler Kontext in meinem Übersetzungsargument vorkommt. Im Übersetzungsargument geht es um die Definierbarkeit des Mentalen in physikalischer Sprache und nicht um den Glauben, ob das Mentale in physikalischer definierbar ist. Deshalb kann beim Übersetzungsargument nicht rationalerweise angenommen werden, dass eine Gehirnaktivität ausschließlich in physikalischer Sprache definierbar ist, aber gleichzeitig ein Furchterlebnis nicht ausschließlich in physikalischer Sprache definierbar ist.

Ein vierter Einwand kommt von Smart (2007, dritter Absatz seines EnzyklopädieEintrages). Er meint, dass eine Übersetzung eines mentalen Vokabulars in ein physikalisches 
Vokabular nicht möglich sei, weil alle Begriffe, die physikalische Entitäten herauspicken, nur durch ein Hindeuten auf die fragliche Entität gelernt werden können. Wenn ich beispielsweise wissen will, was der Begriff "Baum" bedeutet, dann wird die Äußerung dieses Begriffes mit einer hindeutenden Geste auf einen Baum vervollständigt. Hier scheint Smart anzunehmen, ohne es jedoch explizit zu sagen, dass das Lernen von mentalen Begriffen anders passiert. Nur unter dieser Annahme kann Smarts Einwand wie folgt weitergeführt werden: Eine Übersetzung von mentalen Begriffen in physikalische Begriffe ist deshalb nicht möglich, weil physikalische Begriffe durch Hindeuten auf den Referenten gelernt werden, aber mentale Begriffe werden nicht durch ein Hindeuten auf den Referenten gelernt. Der Begriff "neuronale Aktivität N" wird durch das Hindeuten auf die neuronale Aktivität $\mathrm{N}$ gelernt, der Begriff "Furcht" jedoch nicht.

Ich denke nicht, dass Smart Recht hat, und meine Replik zielt auf die vermeintliche Unterscheidung des Referenzaktes zwischen physikalischen und mentalen Begriffen ab.

Nehmen wir an, dass die Identität zwischen der mentalen und physikalischen Entität noch nicht bekannt ist. Der physikalische Begriff "P" wird durch ein Hindeuten auf eine Sache, ein Ding, eine Eigenschaft, eine Relation oder irgendeine andere physikalischen Entität erlernt, womöglich auch begleitet mit einer entsprechenden verbalen Äußerung. Diese physikalische Entität ist Teil der subjektunabhängigen, objektiven Welt. Nun sehen wir uns den mentalen Begriff " $M$ " an. Warum sollte dieser nicht auch durch ein Hindeuten auf eine Sache, ein Ding, eine Eigenschaft, eine Relation oder irgendeine andere mentale Entität erlernt werden? Das Hindeuten passiert bei M zwar nicht durch eine demonstrative Geste wie einer Handbewegung oder dergleichen, sondern durch die korrelierenden Variablen wie das Aufreißen des Mundes bei Verwunderung, das Zusammenrollen des Oberkörpers bei Bauchschmerzen und alle anderen Prozesse, die dafür geeignet sein können. Diese korrelierenden Variablen sind wiederum Teil der subjektunabhängigen, objektiven Welt. Selbstverständlich kann dieser Akt des Hindeutens auch mit verbalen Äußerung versehen werden, wie beispielsweise ein "Ohhhh" bei Verwunderung und ein "Auweh" bei Bauchschmerzen.

\subsection{Das Mentale und Physikalische: eine Definition deren Relation Teil 1}

Die Analysen aus Kapitel 3 und 4 führen uns schließlich zu einer Definition des Teiles "alles" bzw. "nichts anderes als" der intuitiven Definition des Physikalismus. Wir sind an einer Stelle angelangt, an der die Relation zwischen dem Mentalen und dem Physikalischen genau benannt und definiert werden kann.

(R1) Eine mentale Entität ist genau dann nichts anderes als eine physikalische Entität, wenn kontingenterweise a priori und analytisch gilt, dass jeder Typus einer mentalen Entität 
a) mit i) dem Typus einer physikalischen Basisentität oder mit ii) dem Typus einer physikalischen Entität höherer Ordnung, die in einer bestimmten Relation zu den physikalischen Basisentitäten steht, ontologisch identisch ist,

und dass jeder Begriff einer mentalen Entität

b) mit dem Begriff i) einer physikalischen Basisentität oder ii) einer physikalischen Entität höherer Ordnung, die in einer bestimmten Relation zu den physikalischen Basisentitäten steht, identisch und damit synonym ist.

In dieser Definition sind alle Erkenntnisse der letzten beiden Kapitel miteingeflossen. Es wird eine ontologische Typen-Identität zwischen dem Mentalen und dem Physikalischen hergestellt sowie ebenso eine begriffliche Identität zwischen mentalen und physikalischen Begriffen. Alle mentalen Begriffe lassen sich ohne Bedeutungsverlust in physikalische Begriffe übersetzen. Außerdem wird eingeräumt, dass das Mentale entweder mit physikalischen Basisentitäten identisch ist, oder mit physikalischen Entität höherer Ordnung, die in einer bestimmten Relation zu den physikalischen Basisentitäten steht, identisch ist. Die Identität zwischen dem Mentalen und dem Physikalischen ist trotz des A-priori-Charakters und des Analytizitäts-Charakters kontingent, weil alles Physikalische davon abhängt, wie unsere aktuale Welt beschaffen ist. Wie unsere aktuale Welt tatsächlich beschaffen ist, ist ein kontingenter Sachverhalt, denn sie hätte auch anders beschaffen sein können. Somit macht dieser kontingente Sachverhalt die TypenIdentität zwischen dem Mentalen und dem Physikalischen ebenso kontingent.

\subsection{Zusammenfassung}

Ich habe dieses Kapitel damit begonnen, die Kriterien der Einfachheit und der Sparsamkeit einzuführen. Sodann habe ich gezeigt, dass einfachere und wenig sparsamere Theorien eine höhere Wahrscheinlichkeit haben, wahr zu sein, als weniger einfache und sparsame Theorien. Dadurch können beide Kriterien als rationale Richtlinien herangezogen werden, um zwischen verschiedenen konkurrierenden Theorien zu entscheiden bzw. darüber zu entscheiden, welcher dieser Theorien der Vorzug zu geben ist. Nachdem ich argumentiert habe, dass ein Supervenienzphysikalismus nicht hinreichend für eine gangbare Bewusstseinstheorie ist, habe ich durch Anwendung der zwei grundlegenden Kriterien der Einfachheit und der Sparsamkeit den Vorzug einer Typen-Identitätstheorie im Vergleich zu konkurrierenden Bewusstseinstheorien herausgearbeitet. Als Haupteinwand gegen eine Typen-Identität des Mentalen und des Physikalischen wird sehr gerne das Argument der multiplen Realisierbarkeit gebracht, das maximal eine Token-Identität bei bestimmten Individuen in einer bestimmten Spezies zu einem bestimmten Zeitpunkt zeigt. Ich habe auf Basis homologer Strukturen über verschiedene 
Spezies hinweg gezeigt, dass eine gewisse Variabilität der Gehirnaktivität nicht gegen eine Typenreduktion spricht. Ein Typus wird dabei als die charakterisierende Eigenschaft der zugehörigen Tokens verstanden. Diese charakterisierende Eigenschaft wird zwischen verschiedenen Spezies in homologen Gehirnstrukturen realisiert. Schließlich führt uns ein konsequentes Weiterdenken der Einfachheit und der Sparsamkeit wissenschaftlicher Theorien zur Erkenntnis, dass die Typen-Identitätstheorie eine Theorie a priori sein muss. Neben dieser ontologischen Reduktion des Mentalen auf das Physikalische habe ich ein Übersetzungsargument entwickelt, das unter Gültigkeit einer Typen-Identitätstheorie aus zwei einfachen und akzeptierten Prämissen auch auf die begriffliche Reduktion mentaler Begriffe auf physikalische Begriffe schließt. Ich verteidige nachfolgend gegen Einwände zu meinem Übersetzungsargument den Standpunkt, dass mentale und physikalische Begriffe nicht bloß koreferentiell, sondern auch synonym sind. Auf diesen Analysen aufbauend habe ich eine Definition vorgelegt, wie die Relation zwischen dem Mentalen und dem Physikalischen definiert werden kann. 


\section{Hey, I, oh, I'm still alive, Hey, oh}

Pearl Jam (1991) 


\section{Der Eliminativismus als stiller Begleiter}

Der Eliminativismus hat seit dem Artikel von Frankish (2016) wieder mehr Beachtung erlebt, diesmal unter dem Begriff des Illusionalismus, der im Wesentlichen die eliminativistischen Grundannahmen teilt. So wie ein Eliminativist behauptet ein Illusionist, dass alles Mentale durch etwas Physikalisches ersetzt wird und wir einer Illusion erliegen, wenn wir glauben, dass es Qualia gäbe. Es sei alles lediglich ein schlechter Zaubertrick, der uns die Existenz des Mentalen bloß eindringlich und überzeugend zu zeigen scheint. Während Frankish den Ansatz verfolgt, dass eine eliminativistische Bewusstseinstheorie das Rennen im Vergleich zu allen anderen physikalistischen Bewusstseinstheorien gewinnt, geht Dennett (2016) einen Schritt weiter und behauptet, dass der Eliminativismus als die Standardtheorie gelten sollte. Nur durch starke Argumente sollte nach Dennett schließlich davon abgewichen werden und rivalisierende Theorien akzeptiert werden. Ich möchte aus dieser neueren Entwicklung heraus in diesem Kapitel die Frage behandeln, ob der Eliminativismus tatsächlich das Rennen gegen die noch erstplatzierte Typen-Identitätstheorie a priori gewinnt. Hierfür werde ich als Erstes die Alltagspsychologie näher beleuchten, die sich im Wesentlichen auf die allgemein verbreiteten und gewöhnlicherweise verwendeten Annahmen bezieht, wie wir das Verhalten und Erleben von uns selbst und von anderen Personen erklären. Beispielsweise erklären wir den Gang zum Kühlschrank gewöhnlicherweise damit, dass wir Hunger oder Durst haben. Als Zweites diskutiere ich die Voraussetzung des Eliminativismus, dass mentale Begriffe letztendlich leere Begriffe ohne einen Referenten sind, so wie es bei den Begriffen "Hexe", "Weihnachtsmann" und dergleichen der Fall ist. In diesem Zusammenhang argumentiere ich gegen Stich (1996) und entwickle eine deskriptivistische Referenztheorie, auf die sich der Eliminativist getrost beziehen kann. Als drittes Ziel entwickle ich einen liberalen Typen-Eliminativismus a priori, wobei sich das Liberale im Eliminativismus auf die Brauchbarkeit eines mentalen Vokabulars bezieht, sobald ein Fiktionalismus akzeptiert wird. Mein viertes Ziel vergleicht meine Version des Eliminativismus mit meiner Version der Identitätstheorie und zeigt, dass der Eliminativismus einfacher und begrifflich sparsamer ist, aber dennoch nicht der Identitätsthese vorzuziehen ist. Dieses Kapitel endet schließlich mit einer Definition des Physikalismus, wie er sich aus den bisherigen Analysen ergibt.

\subsection{Die Grundlagen des Eliminativismus}

Der Eliminativismus kommt nicht ohne bestimmte Grundlagen aus, die im Kern dieser Position sind. Es sind im Wesentlichen zwei miteinander zusammenhängende Punkte. Einerseits spreche ich damit die Altagspsychologie an, die sich als falsch erweisen muss, und andererseits eine Referenztheorie, die mentale Begriffe zu leeren Begriffen macht. Doch zuerst möchte ich drei Einwände gegen den Eliminativismus diskutieren, von denen zwei üblicherweise in der 
Literatur vorgebracht werden und einer aus dem Kontext der vorliegenden Arbeit konstruiert werden könnte.

\subsubsection{Einwände und Repliken gegen den Eliminativismus}

Oftmals wird der Eliminativismus durch ein kurzes Kommentar vermeintlich widerlegt, weil der Eliminativist behauptet, dass es keine mentalen Phänomene gibt, er aber gleichzeitig seine eigene Behauptung glauben muss und es sich weiterfolgend in gewisser Weise anfühlt, in einem Zustand des Behauptens zu sein. Der erste Vorwurf an den Eliminativist ist somit, dass er sich durch die Wahrheit seiner These selbst widerlegt. Ich denke, dass dieser Einwand leicht entkräftet werden kann. Der Eliminativist muss sich nur auf eine finale physikalische Theorie beziehen, die unsere Theorien über das Mentale vollständig ersetzt. So mag es vielleicht sein, zum Zwecke des Arguments, dass der Eliminativismus in Bezug auf gegenwärtige physikalische Theorien, die das Mentale noch nicht vollständig ersetzen, selbstwiderlegend erscheint. Doch wird eine finale physikalische Theorie herangezogen, dann verschwinden gegenwärtige mentale Entitäten wie eine "Behauptung" sowie ein "Behauptungs-Quale" und der Eliminativismus erscheint nicht mehr selbstwiderlegend. Der Grund dafür ist, dass unsere gegenwärtigen alltagspsychologischen Theorien sowie deren Vokabular und die postulierten Entitäten fehlgeleitet sind, damit sind auch Einwände auf Basis dieser alltagspsychologischen Theorien, deren Vokabular und die postulierten Entitäten fehlgeleitet. Der Vorwurf einer Selbstwiderlegung und dadurch einer Inkohärenz ist somit nicht mehr anwendbar.

Ein zweiter Einwand wird oftmals in einer Fußnote oder in einem kurzen Nebensatz gebracht und betrifft den Vorwurf, dass der Eliminativismus selbstevidente Tatsachen schlichtweg verneint. Wir alle haben tagtäglich Wünsche, Bedürfnisse und viele diverse Empfindungen und Erlebnisse, so der Einwand, die kaum ernsthaft verneint werden können. Wenn eine Bewusstseinstheorie dieses Offensichtliche verneint, dann kann man sie getrost zur Seite legen. Tatsächliche Argumente, die diesen Einwand stützen, finden sich jedoch nicht in der Literatur. So schreibt beispielsweise Chalmers Mitte der 90er-Jahre (Chalmers, 1996): "Eliminativism about conscious experience is an unreasonable position only because of our own acquaintance with it " (S. 90) oder auch "For myself, [...] eliminativism seems so clearly false" (S. 150) oder auch "... all the problems that eliminativism naturally faces. In particular, it denies the evidence of our own experience" (S. 175). Die Stoßrichtung der Argumentation hat sich bei Chalmers auch nach mehr als 20 Jahren nicht wesenlich verändert (Chalmers, 2018, S. 54): "That said, I think illusionism is obviously false, because it is obvious that people feel pain". Zusammengefasst, und Chalmers ist lediglich ein Beispiel von vielen, wird der Eliminativismus nur deshalb als falsch beurteilt, weil er eine kontraintuitive Position ist. Doch ist Intuition alles? Ich denke nicht, und zitiere Frankish (2016, S. 37), der in aller Klarheit und treffend formuliert: "The question is not whether illusionism is intuitively plausible, but whether it is rationally 
compelling". Ob der Eliminativismus auch tatsächlich aus rationalen Gründen akzeptiert werden soll, ist eine wesentlich andere Frage als die Frage, ob der Eliminativismus intuitiv einleuchtend ist.

Ein dritter Einwand betrifft den Eliminativismus im Kontext dieser Arbeit, insbesondere in Bezug auf mein Argument aus Kapitel 2, das auf eine hinreichende Parallelität zwischen physikalischen und mentalen Theorien abzielt, um damit gegen die Via Negativa zu argumentieren. So könnte man sagen, dass ich mir selbst widerspreche, weil ich einerseits in Kapitel 2 die Vergleichbarkeit zwischen physikalischen und mentalen Theorien hergestellt habe und andererseits an dieser Stelle den Eliminativismus verteidige, der die mentalen Theorien als falsch bewertet. Meine Replik zielt auf die unbedingte Voraussetzung der Via Negativa ab, dass ein Verständnis des Mentalen überhaupt gegeben sein muss. Falls nun der Eliminativismus wahr ist, dann ist jedoch der Via Negativa jegliches Verständnis von etwas Mentalem geraubt. Das ist deshalb der Fall, weil das intuitive Verständnis von etwas Mentalem von Grund auf fehlgeleitet ist, weil jegliche mentalen Begriffe keinen Referenten haben, und deshalb eine finale Physik die Alltagspsychologie verdrängt. Ohne die Möglichkeit, überhaupt ein eigenständiges Verständnis des Mentalen zu erlangen, verliert auch die Via Negativa jegliche Daseinsberechtigung und ist in diesem Szenario ein Nicht-Starter. In weiterer Folge ist es auch nicht mehr notwendig, gegen eine Via Negativa auf Basis einer hinreichenden Parallelität zwischen physikalischen und mentalen Theorien zu argumentieren. Dadurch ist es einfach zu sehen, dass kein Widerspruch zum bisher Gesagten vorliegt, falls der Eliminativismus wahr ist.

\subsubsection{Was ist die Alltagspsychologie?}

Wir alle haben eine Vorstellung davon, warum wir etwas machen. Wir treffen tagtäglich Annahmen, warum wir genau so handeln, wie wir handeln. Unsere Annahme ist, dass in den meisten Situationen unsere mentalen Zustände wie beispielsweise unser Wille, unsere Hoffnung, ein positives oder ein negatives Gefühl die Auslöser sind. Ich will einen Waldspaziergang machen, deshalb tue ich es und fühle Zufriedenheit, sobald ich mich zwischen den Bäumen bewege. Ich hoffe, dass der Supermarkt pünktlich aufsperrt, deshalb bin ich zur angegeben Öffnungszeit am Eingangstor. Ich empfinde Liebe, deshalb wünsche ich mir alles erdenklich Gute für meine Partnerin. Schlussendlich löst eine Furcht vor Hunden mein Wechseln der Straßenseite aus, gefolgt von einem Gefühl der Sicherheit. Neben diesen Erklärungen von Verhalten und Erleben können wir auch das Verhalten und Erleben anderer vorhersagen. Beispielsweise treffe ich die Vorhersage, dass mein Arbeitskollege jeden Morgen gegen 8 Uhr in die Kaffeeküche geht und nach 2 Minuten mit einem Espresso und einem Glas Wasser den Weg zurück in sein Büro mit zufriedener Miene antritt. Ich habe mit dieser Vorhersage nicht immer recht, doch ich behalte in den überwiegenden Fällen recht. Ohne eine psychologischwissenschaftliche Theorie zu kennen, manövriere ich mich durch meine Umgebung und wende dieselben gewöhnlichen psychologischen Begriffe, Konzepte, Annahmen und 
Gesetzmäßigkeiten an. Ich erkläre das Verhalten und Erleben von mir, aber auch von anderen, und kann mit einer überzufälligen Trefferquote das Verhalten und Erleben von mir und den anderen vorhersagen. Genau das ist die Alltagspsychologie, die voll ist mit Bezügen auf eine Vielzahl an kausal wirksamen mentalen Zuständen und die anscheinend in unserem alltäglichen Leben mit Erfolg Erklärungen und Vorhersagen leistet.

Der Eliminativismus ist nun auf eine ganz bestimmte Entwicklung der Alltagspsychologie angewiesen, um für seine Position überhaupt erst argumentieren zu können. Die finalen alltagspsychologischen Begriffe, Konzepte, Annahmen und Gesetzmäßigkeiten dürfen keine Entsprechungen in einer finalen physikalischen Theorie und in deren Begriffen, Konzepten, Annahmen und Gesetzmäßigkeiten finden. Warum solch eine Entsprechung nicht gegeben ist, verleiht nach Wallace (2016) dem Eliminativismus wiederum verschiedene Nuancen. So würden einige Entitäten der Altagspsychologie bereits einen Dualismus voraussetzen (z.B. Feyerabend, 1963) oder sie seien zu vage und unbestimmt (z.B. Churchland, 1981) oder sie seien in sich widersprüchlich (z.B. Dennett, 1988) oder die gesamte Alltagspsychologie entwickelt sich schlichtweg nicht weiter und stagniert (z.B. Churchland, 1981). Was auch immer der präferierte Grund gegen die Alltagspsychologie ist, der Eliminativist muss einen liefern. Diese klassischen Quellen der eliminativistischen Literatur schließen durch die fehlende Entsprechung der finalen Alltagpsychologie mit einer finalen Physik auf die Falschheit der Alltagspsychologie. Wenn diese nun falsch ist, dann gibt es auch die in der Alltagspsychologie postulierten Entitäten nicht mehr. Vermeintlich existierende mentale Entitäten sind letztendlich lediglich auf gleicher Stufe wie eine Hexe, ein Phlogiston, ein Einhorn und der Weihnachtsmann. Nichts von denen existiert tatsächlich und die Begriffe, die auf diese Dinge referieren, sind leere Begriffe ohne einen Referenten.

\subsubsection{Der Status der Alltagspsychologie: Reduktion vs. Elimination}

Wie können wir nun das Schicksal der Alltagspsychologie in Erfahrung bringen? Meine Antwort ist, dass wir das nicht eindeutig können. Es mag sein, dass die gegenwärtige Alltagspsychologie in alltäglicher Erklärung und Vorhersage unseres menschlichen Verhaltens zumindest zweckdienlich und brauchbar ist. Ob eine finale und vollendete Altagspsychologie genau so sein wird oder doch, wie von Eliminativisten behauptet, radikal falsch sein wird, ist schwierig zu prophezeien. Aber auch der gegenteilige Ausgang ist nicht eindeutig festzumachen, nämlich, dass sie eine wahre Theorie sein wird, die eine Entsprechung in einer finalen, vollendeten Physik finden wird. Als Physikalist müssen wir jedoch eines der beiden Optionen annehmen.

Ich habe mich in den Kapiteln zuvor auf eine Reduktion des Mentalen auf das Physikalische verpflichtet, meine Argumente für diese Position waren durch Überlegungen zur Einfachheit und Sparsamkeit der konkurrierenden physikalistischer Theorien geprägt. Den Vergleich hat schlussendlich die A-priori-Identitätstheorie gewonnen. In diesem Kapitel blicke ich 
nochmals auf meine Argumentation mit der Brille eines Eliminativisten. Umso erstaunlicher ist es, dass im Lager der Eliminativisten ein ganz klares Bekenntnis gegen einen reduktionistischen Ansatz fehlt. Es wird oftmals das Verhältnis zwischen einer ontologischen Reduktion und einer Elimination weniger dogmatisch für die eine oder andere Richtung dargelegt. Beispielsweise schreibt Churchland (1981, S. 75), dass sich eine Reduktion letztendlich doch nicht völlig eindeutig ausschließen lässt:

A successful reduction cannot be ruled out, in my view, but FP's [folk psychology's] explanatory impotence and long stagnation inspire little faith that its categories will find themselves neatly reflected in the framework of neuroscience. On the contrary, one is reminded of how alchemy must have looked as elemental chemistry was taking form, how Aristotelian cosmology must have looked as classic mechanics was being articulated, or how the vitalist conception of life must have looked as organic chemistry marched forward.

Einige Jahre später akzeptiert Churchland sogar explizit die Reduktion von Qualia und behält sich eine Elimination von intentionalen Zuständen vor (Churchland, 2007, S. 166):

However, in one important area, a blanket eliminative materialism bids fair to be just plain wrong. The reason is simple: The portion of folk psychology concerned with the various sensations to which we are subject is in the process of finding a moderately smooth and highly illuminating reduction at the hands of unfolding neuroscience.

Alles in allem wird durch diese Veränderungen der wissenschaftlich gespeisten Sichtweisen deutlich, also von der Elimination hin zur Reduktion, dass sich der eliminative Physikalismus und der reduktionistische Physikalismus nicht gegenseitig ausschließen. Es kann sehr wohl sein, dass ein Bereich des mentalen Lebens einer Reduktion zugänglich wird und ein anderer Bereich einer Elimination zum Opfer fällt. Ebenso kann es sein, dass innerhalb eines Bereiches, beispielsweise im Bereich der Qualia, einige Erlebnisinhalte reduziert werden können und anderer wiederum eliminiert werden. Dasselbe könnte auch für intentionale Zustände gelten. Mir geht es darum, zu zeigen, dass je nach Entwicklung der Alltagspsychologie und je nach Entwicklung der Naturwissenschaften all diese Ausgänge möglich sind. Als Konsequenz daraus spreche ich mich auch für eine disjunktive Erweiterung jeder Formulierung eines reduktiven Physikalismus um den Eliminativismus aus. Alle mentalen Entitäten, die nicht reduziert werden können, werden eliminiert und umgekehrt. Die Unmöglichkeiten, das Schicksal der Alltagspsychologie ein für alle Mal vorherzusagen, hat uns zu dieser Konklusion geführt. 


\subsubsection{Haben mentale Begriffe einen Referenten?}

Das, worauf sich ein Begriff bezieht, ist sein Referent. Wenn ich von einem Tisch spreche, bezieht sich der Begriff "Tisch" auf diesen Gegenstand als etwas Existierendes, und wenn ich von einem Schmerz spreche, dann bezieht sich auch der mentale Begriff "Schmerz" auf etwas real Existierendes. Wie der Ausdruck "real existierend" gedeutet wird, hängt vom entsprechenden Lager ab, zu dem sich der Philosoph zugehörig fühlt. Für Dualisten existieren Schmerzen zusätzlich zu körperlichen Vorgängen, für Identitätstheoretiker existieren Schmerzen als nichts anderes als körperliche Vorgänge und für Eliminativisten wird die Existenz von Schmerzen geleugnet und durch die Existenz von körperlichen Vorgängen ersetzt. Abseits davon ist für die gegenwärtigen Zwecke die Art und Weise der Referenz von Interesse. Ob nun der Referent eines Begriffes auch gleichzeitig dasselbe wie die Bedeutung des Begriffes ist, hat Frege (1892) überzeugend und einflussreich verneint. Er argumentiert, dass die Referenz (in seinen Wörtern die Bedeutung, im Sinne eines Hindeutens) und die Bedeutung (in seinen Wörtern der Sinn) von Begriffen getrennt werden müssen. Frege betrachtet folgendes Beispiel anhand von singulären Termen:

(1) Morgenstern $=$ Morgenstern

(2) Morgenstern $=$ Abendstern

Sowohl der Morgenstern als auch der Abendstern haben denselben Referenten, nämlich die Venus. Gleichzeitig ist jedoch Aussage (1) trivial und eine logische Wahrheit, Aussage (2) aber eine kognitiv signifikante Entdeckung, die durch empirische Forschung herausgefunden wurde. Frege zeigt anhand dieses Beispiels, dass der Morgenstern und der Abendstern dieselbe Referenz, aber eine verschiedene Bedeutung haben. Bezüglich Referenz und Bedeutung in der Diskussion um den Eliminativismus schreibt Stich (1996, S. 85) in Endnote 29: "Since reference rather than meaning does all the work in eliminativists' arguments, I won't have much to say about theories about the meaning of theoretical [i.e., mental] terms". Da in der Argumentation des Eliminativisten die Referenz wesentlich ist, aber nicht die Bedeutung, werde ich mich nachfolgend ausschließlich auf die Referenz mentaler Begriffe beschränken.

Frege ist ebenso gemeinsam mit anderen Philosophen wie Russell (1905) Vertreter einer deskriptivistischen Referenztheorie bzw. einer Kennzeichnungstheorie von Begriffen. Die zentrale Annahme deskriptivistischer Theorien ist die, dass Begriffe versteckte Beschreibungen sind. Betrachten wir Freges Beispiel nun von dieser Warte aus:

(3) Morgenstern = der hellste Stern in der Morgendämmerung

(4) Abendstern = der hellste Stern in der Abenddämmerung 
Der Begriff "Morgenstern" stellt eigentlich eine abgekürzte Version der Beschreibung "der hellste Stern in der Morgendämmerung" dar, genauso wie der Begriff "Abendstern" die Beschreibung "der hellste Stern in der Abenddämmerung" darstellt. Jegliche Referenz im Rahmen eines Frege-Russell-Ansatzes kommt aufgrund bestimmter Zuschreibungen des Referenten zustande.

Wie Stich (1996) seine Kritik am Eliminativismus weiter ausführt, muss der Eliminativist eine deskriptivistische Referenztheorie annehmen, damit für mentale Begriffe der Altagspsychologie nichts gefunden wird, das der Beschreibung entspricht. Wenn beispielsweise der Begriff "Hexe" für eine Frau steht, die mit dem Teufel einen Pakt geschlossen hat, es aber einen Teufel und dementsprechend einen Pakt nicht gibt, dann kann auch für den Begriff "Hexe" nichts gefunden werden, das der Beschreibung "ist eine Frau und hat einen Pakt mit dem Teufel geschlossen" entspricht. Somit existieren keine Hexen. Wenn der mentale Begriff "Schmerz" mit etwas beschrieben wird, das sich persönlich, individuell, selbstevident und irrtumssicher negativ anfühlt, sich in den Naturwissenschaften aber nichts finden lässt, das der Beschreibung "ist persönlich, individuell, selbstevident, irrtumssicher und fühlt sich negativ an" entspricht, dann gibt es auch keinen Schmerz.

Dem gegenüber steht die kausal-historische Referenztheorie von Begriffen, die aus der Kritik am Deskriptivismus hervorgegangen ist (Kripke, 1980). Nach Kripke liegt das Problem des Deskriptivismus unter anderem darin, dass eine Eigenschaftszuschreibung zu Personen nicht die Referenz auf genau dieselbe Person garantiert. Sehen wir uns diesen Punkt näher an:

(5) Gödel = der Urheber der Unvollständigkeitstheoreme

Der Begriff "Gödel" referiert nur dann auf Kurt Gödel, wenn auch Kurt Gödel die Unvollständigkeitstheoreme verfasst hat. Aber wie wollen wir das genau wissen? Kripke sagt, dass auch eine unbekannte Person die Unvollständigkeitstheoreme verfasst und Gödel von dieser Person abgeschrieben haben könnte. Damit würde der Begriff "Gödel" nicht wirklich auf Kurt Gödel referieren, sondern eigentlich auf die unbekannte Person, weil eben diese die Unvollständigkeitstheoreme verfasst hat. Falls eine deskriptivistische Referenztheorie wahr wäre, dann würde dieser Wechsel des Referenten jedoch nicht möglich sein und "Gödel" immer noch fälschlicherweise Kurt Gödel als Referenten haben. Es existiert nach Kripke also eine mögliche Welt, in welcher der Begriff "Gödel" nicht auf Kurt Gödel referiert.

Kripke löst dieses Problem dadurch, indem er einen Taufakt vorschlägt, in dem ein Begriff von einem Sprecher einen kausal verbundenen Referenten zugewiesen bekommt. So könnte der Sprecher sagen, dass genau diese Person, die vor mir sitzt, von nun an Gödel heißt und mit "Gödel" genau auf diese Person referiert wird. In weiterer Folge wird von einem Sprecher zum nächsten und über die Jahre hinweg der Referent von "Gödel" immer weitergegeben, sodass diese Kette des Weitergebens den ursprünglichen Referenten des 
Begriffes "Gödel" erhält. In dieser kausal-historischen Referenzgebung ist ein Referieren auf eine andere Person als auf Kurt Gödel nicht möglich und "Gödel" fungiert als ein starrer Bezeichnungsausdruck, der in allen möglichen Welten Kurt Gödel herauspickt. Ein Referenzfehler, sodass "Gödel" nicht auf Kurt Gödel referiert, ist deshalb nicht möglich, weil der Referent durch den Taufakt zuvor bereits festgelegt wurde. Genauso lässt sich auch die Analyse auf mentale Begriffe wie beispielsweise "Schmerz" übertragen. Wenn ich mich in diesem persönlichen, individuellen, selbstevidenten und irrtumssicheren Zustand befinde, der sich negativ anfühlt, und ich diesen Zustand "Schmerz" taufe, dann pickt auch "Schmerz" in allen möglichen Welten diesen Zustand heraus. Schmerz und alle anderen mentalen Begriffe fungieren somit ebenfalls als starre Bezeichnungsausdrücke. Dass nun ein mentaler Begriff keinen Referenten hätte, ist im kausal-historischen Ansatz somit nicht möglich. Das stellt zweierlei Problem dar. Zum einen hat der Eliminativist keine Chance, leere Begriffe der Alltagspsychologie zu bestimmen, zum anderen ist es ein offensichtliches Problem des kausalhistorischen Ansatz, für keine leeren Begriffen eine schnelle Erklärung zu liefern. Aus diesen Gründen ist diese Art einer Referenztheorie für den Eliminativisten nicht gangbar.

\subsubsection{Warum der Deskriptivismus gangbar ist}

Wenn nun, so wie Stich (1996) argumentiert, eine deskriptivistische Theorie eine Voraussetzung ist, um in der Alltagspsychologie leere Begriffe bestimmen zu können, muss von Vertretern des Eliminativismus solch eine Theorie vorgelegt werden. Diese deskriptivistische Theorie muss auch gleichzeitig die klassischen Probleme einer fehlerhaften Referenz umgehen, wie sie weitreichend von Kripke (1980) vorgebracht wurden. So wie ich Kripke verstehe, ist sein höheres Ziel, eine Referenztheorie für Begriffe zu entwickeln, die in allen möglichen Welten gilt. Starre Bezeichnungsausdrücke picken notwendigerweise immer dasselbe heraus, unabhängig davon, ob wir die Referenz in der aktualen Welt oder irgendeiner anderen möglichen Welt betrachten. Vielleicht hat Kripke Recht, dass dieses Ziel nicht durch eine deskriptivistische Referenztheorie erreicht werden kann. Ob dies auch tatsächlich der Fall ist, darauf möchte ich nicht eingehen. Stattdessen möchte ich die Voraussetzung bei Kripke angreifen, dass die Referenz eines Begriffes über alle möglichen Welten hinweg fixiert werden muss.

Ich möchte an dieser Stelle für die These argumentieren, dass es für die Zwecke des Eliminativismus ausreicht, wenn die Referenz eines Begriffes in der aktualen Welt und in allen hinreichend ähnlichen möglichen Welten fixiert werden kann. Hierfür möchte ich das Ziel des Physikalismus nochmals in Erinnerung rufen. Der Physikalismus, dessen Spielart immerhin die des Eliminativismus darstellt, hat die Erklärung des Zusammenhanges zwischen dem Mentalen und dem Physikalischen als Ziel. Die minimale Voraussetzung dabei ist, dass eine Supervenienzrelation zwischen mentalen und physikalischen Entitäten besteht. Es ist weitläufig in der Philosophie des Geistes akzeptiert, und das gerechtfertigterweise, dass in einer 
Supervenienzformulierung des Physikalismus eine Art Stopp-Zeichen eingefügt werden muss, sei es nun in der Spielart von Jackson (1998a) oder Chalmers (1996). Dadurch ist erreicht, dass die Supervenienzrelation in der aktualen Welt und in allen hinreichend ähnlichen möglichen Welten gilt, die ich Jackson folgend als minimale physikalische Duplikate unserer Welt bezeichne. Nichtsdestotrotz untergräbt eine mögliche Welt, die komplett unähnlich zu unserer aktualen Welt ist und in der Geister, cartesische Seelen und Hexen existieren, nicht die Wahrheit des Physikalismus. Das Stopp-Zeichen sichert die Wahrheit des Physikalismus, nämlich genau in denjenigen Welten, die für uns von Interesse sind, und lässt die Falschheit des Physikalismus in genau denjenigen Welten zu, die für uns nicht von Interesse sind. Warum sollten für eine Referenztheorie unnötigerweise strengere Maßstäbe auferlegt werden? Ein Einfügen eines Stopp-Zeichens würde auch hier die vorliegenden Probleme lösen und die Referenz eines Begriffes auf die aktuale Welt und auf minimale physikalische Duplikate beschränken. Eine mögliche Version dieses Stopp-Zeichens könnte in Jacksons Vokabular aus Kapitel 3 wie folgt aussehen. Es sei angemerkt, dass getrost jede andere Formulierung eines Stopp-Zeichens verwendet werden kann und nicht ausschließlich Jacksons Formulierung voraussetzt:

(Referenz-Stopp) Jeder Referent eines Begriffes, der ein minimales Referenz-Duplikat aus unserer Welt darstellt, ist ein Referent schlechthin.

Der Ausdruck "minimales Referenz-Duplikat" bedarf noch weiterer Erläuterungen. Eine Referenz in einer möglichen Welt W1 ist genau dann ein minimales Referenz-Duplikat aus unserer Welt $\mathrm{W}$, wenn es (a) exakt wie in $\mathrm{W}$ in allen referenzfixierenden Eigenschaften gleich ist und (b) keine anderen referenzfixierenden Eigenschaften enthält, die es enthalten muss, um (a) gerecht zu werden. Die Konsequenz von (Referenz-Stopp) ist die, dass Vertreter einer deskriptivistischen Referenztheorie die Möglichkeit einer falschen Referenz erlauben und die referenzfixierende Eigenschaft "ist der Autor der Unvollständigkeitstheoreme" nicht auf Kurt Gödel, sondern auf eine andere Person referiert, von der eben Kurt Gödel abgeschrieben hat. Nennen wir diese andere Person Kurt Schmidt. Der deskriptivistische Referenztheoretiker kann es deshalb erlauben, weil Kurt Gödel als vermeintlicher und unechter Autor der Unvollständigkeitstheoreme in W1 kein minimales Referenz-Duplikat aus W ist. Falls aber nun Kurt Gödel auch in W1 der tatsächliche und echte Autor der Unvollständigkeitstheoreme wäre, dann wäre Kurt Gödel in W1 ein minimales Referenz-Duplikat aus W. Durch (Referenz-Stopp) kann nun eine deskriptivistische Referenztheorie vertreten werden, die nicht in allen möglichen Welten Gültigkeit hat, sondern nur in der aktualen Welt und in hinreichend ähnlichen möglichen Welten. 


\subsection{Mentale Begriffe im Eliminativismus}

Nachdem der Eliminativismus jegliche Formulierung eines reduktiven Physikalismus begleitet, bleibt die Frage offen, wie mit den leeren Begriffen umgegangen werden soll. Ich möchte zur Diskussion dieser Thematik die Unterscheidung von Machery (2009) anwenden, nämlich diejenige, die zwischen einem traditionellen Eliminativismus und einem wissenschaftlichen Eliminativismus unterscheidet. Traditionelle Eliminativisten haben eine Antwort auf die Frage der Verwendung leerer Begriffe: Sie sollten nicht mehr verwendet und sollten eliminiert werden, sowohl im wissenschaftlichen als auch im alltäglichen Diskurs, genauso wie der vermeintliche Referent des Begriffes eliminiert wurde. Hingegen verteidigen wissenschaftliche Eliminativisten den Standpunkt, dass leere mentale Begriffe aus dem wissenschaftlichen Diskurs eliminiert werden sollten, sie sind jedoch neutral, was die Begriffsverwendung im alltäglichen Diskurs betrifft. Corns (2016) greift diese Zweiteilung des Eliminativismus auf und wendet inn konkret auf den Begriff "Schmerz" an. Zuerst gibt sie eine Zusammenfassung der momentan in den Wissenschaften anerkannten Definition des Schmerzes durch Bezug auf die International Association for the Study of Pain (IASP), welche Schmerzen immer als ein subjektives Erlebnis definiert, das in einem variierenden Ausmaß durch biologische, psychologische und soziale Faktoren beeinflusst wird ${ }^{46}$. Sodann diskutiert Corns Ansätze von traditionellen QualiaEliminativisten wie beispielsweise Daniel Dennett und deren Sichtweisen zu Schmerzen, wobei das Ergebnis nicht verwundert und in einer ontologischen und begrifflichen Elimination des Schmerzes mündet.

Ein wesentlicher Beitrag Corns zur Debatte rund um den Eliminativismus ist die genauere Analyse des Abhängigkeitsverhältnisses zwischen der traditionellen und der wissenschaftlichen Spielart. Zuallererst sei angemerkt, dass Corns, so wie ich ihre Argumentation verstehe, sowohl den traditionellen als auch den wissenschaftlichen Eliminativismus als voneinander unabhängige Standpunkte sieht, zumindest in folgender Art und Weise. Sie spricht sich klar dagegen aus, dass ein traditioneller Eliminativismus aus der Akzeptanz eines wissenschaftlichen Eliminativismus folgt, und man kann daher konsistent wissenschaftlicher Eliminativist sein, ohne auch gleichzeitig traditioneller Eliminativist zu sein ${ }^{47}$. Um für ihren Standpunkt zu argumentieren, bringt Corns zwei Beispiele. Sie lädt den Leser ein, die Begriffe "Dienstag" und "Freunde" zu betrachten. Nach Corns sind beide Begriffe in unserem Alltag verhaftet und wir verwenden beide Begriffe, womöglich in einem Satz wie "Ich treffe meine Freunde am Dienstag". Gleichzeitig kommen beide Begriffe in keiner Naturwissenschaft vor.

\footnotetext{
46 Siehe vor allem das IASP-Glossar: https://www.iasp-pain.org/Education/Content.aspx?/temNumber=1698\#Pain [05.07.2021].

${ }^{47} \mathrm{Ob}$ auch der umgekehrte Schluss zulässig ist, nämlich, dass ein traditioneller Eliminativist auch konsistenterweise kein wissenschaftlicher Eliminativist sein kann, wird von Corns nicht explizit diskutiert. Diese Frage ist zwar interessant, hat jedoch für die vorliegende Diskussion keine große Relevanz. Der Grund ist, dass typischerweise alle traditionellen Eliminativisten leere Begriffe aus jeglichem Diskurs streichen. Ob traditionelle Eliminativisten auch die Position einnehmen können, leere Begriffe nur aus dem alltäglichen Diskurs zu streichen, aber nicht aus dem wissenschaftlichen Diskurs, bliebe, wenn überhaupt, lediglich eine theoretische Option.
} 
Ähnlich sieht es Corns auch mit mentalen Begriffen wie "Schmerz", die ihre Brauchbarkeit im Alltag behalten, aber im wissenschaftlichen Kontext abgeben.

Um bei unserer Diktion der leeren Begriffe zu bleiben, behauptet Corns, dass "Dienstag" und "Freunde" leere Begriffe sind, aber dennoch ihre Brauchbarkeit im Alltag behalten. Auch wenn Dienstage und Freunde, genauso wie Schmerzen, nicht wirklich existieren, gibt es sie im ausreichenden Maße für alltägliche Belange (Corns, 2016, S. 2968):

I am happy to conclude that the ontological status of pains ... is as robust as that of Tuesdays. One may say that there are not really any Tuesdays if they like, but Tuesdays are real enough for me and for my everyday purposes.

An dieser Stelle drängen sich zwei wesentliche Fragen auf. Einerseits kann hinterfragt werden, ob Dienstage und Freunde nicht doch in einem gewissen Abhängigkeitsverhältnis zu den Naturwissenschaften stehen, sodass deren physikalistische Natur gesichert ist und die Begriffe "Dienstag" und "Freunde" einen Referenten haben. Andererseits ist die Frage offen, was es bedeutet, wenn etwas wirklich existiert und wie wir uns auf etwas Nicht-Existierendes beziehen können. Beide Punkte möchte ich nachfolgend behandeln.

\subsubsection{Die Brauchbarkeit leerer Begriffe in einem liberalen Eliminativismus}

Ich denke, dass Corns Recht hat, wenn sie meint, dass leere Begriffe dennoch im Alltag brauchbar sind. Für mich geht Corns sogar zu wenig weit, wenn sie nur die Brauchbarkeit im Alltag zeigt, denn leere Begriffe können auch in den Wissenschaften eine gewisse Brauchbarkeit aufweisen. Die Spielart des Eliminativismus, der ontologisch alles Mentale eliminiert, aber begrifflich das Mentale im Alltag und in den Wissenschaften als praktikabel akzeptiert, möchte ich als einen liberalen Eliminativismus bezeichnen und hier entwickeln. Ich bin überzeugt, dass Corns Begriffe "Dienstag" und "Freunde" das nicht zeigen, sondern von anderer Seite herangegangen werden sollte. Meine Beweggründe, warum ich nicht von ihren Beispielen überzeugt bin, trifft die Voraussetzung ihrer Argumentation. Corns sagt deutlich, dass Dienstage und Freunde nicht Teil des naturwissenschaftlichen Kanons sind. Dienstage und Freunde wären nach Corns nicht-physikalische Tatsachen und würden so, streng genommen, den Physikalismus gar falsifizieren. Solch eine Position ist zwar prinzipiell möglich, doch unnötig streng. Ein ähnliches Beispiel hat Stoljar (2010, S. 30) in seiner Diskussion des Physikalismus aufgegriffen, um zu zeigen, dass die Formulierung des Physikalismus angepasst werden muss:

The Supreme Court is not a physical object in the ordinary sense, nor has it anything much to do with physics. It seems odd, to put the point a different way, to interpret physicalism so that a physicalist might be refuted by the 
existence and nature of the Supreme Court... Instead it should be interpreted as having a restricted reading of some kind.

Eine Möglichkeit, den Physikalismus in Bezug auf Dienstage und Freunde zu verstehen, ist eine bestimmte Art einer Relation zwischen Dienstage und Freunde auf der einen Seite sowie den naturwissenschaftlichen Postulaten auf der anderen Seite anzunehmen. Dieses Vorgehen erinnert an die Art und Weise, wie wir begonnen haben, das Verhältnis zwischen dem Mentalen und dem Physikalischen zu bestimmen. Nur hier ist es etwas einfacher, weil für den Zweck der Argumentation nicht alle Möglichkeiten durchgegangen werden müssen, wie wir es bei der Diskussion des Qualia-Problems gemacht haben. Es reicht zu zeigen, dass Dienstage und Freunde in einer liberaleren Leseart des Physikalismus auch als Teil der Naturwissenschaften gelten können. Hierfür werde ich die Supervenienzrelation verwenden, um meinen Punkt klar darzulegen. Vielleicht muss die Supervenienzrelation in bestimmter Art und Weise weiter entwickelt werden oder sogar eine Superdupervenienzrelation daraus entstehen. Über diese potentiellen Weiterentwicklungen möchte ich in diesem Kontext nichts sagen und beschränke mich auf die Grundaussagen.

Nehmen wir "Dienstag" als erstes Beispiel. Dienstage haben dann einen Platz in den Naturwissenschaften, wenn sie auf zeitrelevanten Fakten, oder seit Einstein besser auf raumzeitlichen Fakten, supervenieren. Diese Fakten sind beispielsweise das Verhältnis der Positionen der Planeten auf ihren Umlaufbahnen in unserem Sonnensystem. Immer dann, wenn unser Sonnensystem zu einem bestimmten Zeitpunkt in einer bestimmten Art und Weise konstituiert ist im Sinne von Planetenpositionen auf den Umlaufbahnen, dann ist ein Dienstag der richtige Wochentag. Ein Dienstag ist unter dieser Sichtweise nichts anderes als ein komplexes Geflecht von Planetenpositionen auf deren Umlaufbahnen. Schließlich referiert der Begriff "Dienstag" auch genau auf diese wiederkehrenden Planetenkonstellationen.

Nehmen wir "Freunde" als zweites Beispiel. Freunde haben dann einen Platz in den Naturwissenschaften, wenn sie auf zwischenmenschlichen Beziehungsfakten supervenieren. Diese Fakten sind beispielsweise der psychologische, physische und soziale Umgang miteinander. Immer dann, wenn ein bestimmtes Zusammentreffen dieser zwischenmenschlichen Beziehungsfakten gegeben ist, dann sind zwei oder mehrere Personen Freunde. Ein Freund ist unter dieser Sichtweise nichts anderes als ein komplexes Geflecht an zusammentreffenden Beziehungsfaktoren zwischen Menschen. Schließlich referiert der Begriff "Freund" auch genau auf diese Beziehungskonstellation.

Kontra Corns ist die Konklusion, dass Dienstage und Freunde sehr wohl einen Platz in den Naturwissenschaften haben, selbstverständlich in einer liberaleren Leseart. Darüber hinaus haben die Begriffe "Dienstag" und "Freunde" auch einen Referenten und sind somit keine leeren Begriffe. Dennoch bin ich überzeugt, dass Corns richtig liegt in ihrer Grundüberzeugung. Werden nämlich andere Begriffe verwendet, die eindeutig leere Begriffe sind, lässt sich deren 
Brauchbarkeit im Alltag leichter festmachen. Ich möchte ein Standardbeispiel aus der eliminativistischen Literatur wählen. Stich (1996) folgend sind Hexen als Frauen definiert, die einen Pakt mit dem Teufel eingegangen sind. Der Begriff "Hexe" ist ein leerer Begriff, weil es nichts gibt, dass zur Beschreibung zutrifft, eine Frau zu sein und gleichzeitig einen Pakt mit dem Teufel zu haben. Es ist einerseits in den Wissenschaften ein leerer Begriff, weil es keine wissenschaftliche Disziplin der Hexenforschung im obigen Sinne gibt. Natürlich gibt es Forschungsrichtungen, die sich beispielsweise mit der Inquisition und den Hexenverbrennungen in Europa auseinandersetzen, doch darin geht es nicht um Frauen, die tatsächlich einen Pakt mit dem Teufel haben, sondern um Frauen, denen vorgeworfen wird, einen Pakt mit dem Teufel haben. Andererseits ist es auch im Alltag ein leerer Begriff, weil wir nicht tatsächlich meinen, dass eine Frau einen Pakt mit dem Teufel hat, wenn wir sie eine Hexe nennen. Vielmehr ist es in den meisten Fällen schlichtweg ein Schimpfwort, aber keinesfalls eine ernstgemeinte Referenz auf Frauen, die mit dem Teufel unter einer Decke stecken.

Der Eliminativist fordert daraufhin dieselbe Analyse für mentale Begriffe wie beim Begriff "Hexe". So sind beispielsweise Schmerzen nach Dennett (1988) als etwas Unaussprechliches definiert, das im Wesentlichen subjektiv ist, nur aus der Erste-Person-Perspektive erfassbar ist, unmittelbar und irrtumssicher vom Subjekt selbst wahrgenommen wird und als mentaler, nicht weiter zerlegbarer, Baustein unseres Bewusstseins fungiert. Es gibt nichts, das dieser Beschreibung entspricht, deshalb ist "Schmerz" ein leerer Begriff. Die Brauchbarkeit für den Alltag wiederum ergibt sich jedoch aus vielerlei miteinander zusammenhängenden Gründen, allen voran aus kulturellen Gründen, aus pädagogisch-erzieherischen Gründen und aus pragmatischen Gründen. Mit kulturellen Gründen meine ich, dass wir Märchen, Geschichten und Mythen tradieren, die voll sind mit leeren Begriffen. Sei es der dämonische Alb, der in unseren Breitengraden für schlechte Träume (Albträume) verantwortlich ist, Werke aus der Literatur, die Geschichten von Harry Potter erzählen, oder das Christkind, das jedes Jahr zu Weihnachten die Geschenke bringt. Mit pädagogisch-erzieherischen Gründen meine ich, dass Religion ein Unterrichtsfach in Schulen ist, und unabhängig von der eigenen Konfession auch andere Weltreligionen besprochen werden. Als Christ glaubt man an die Existenz des christlichen Gottes, gleichzeitig muss man die Nicht-Existenz der hinduistischen Götter, von Allah und dergleichen glauben. Außerdem wird das Denken und die Phantasie geschürt, wenn sich Kinder überlegen, was sie tun können, damit sie das ganze Jahr über brav sind, um vom Nikolaus Geschenke zu bekommen. Mit pragmatischen Gründen meine ich, dass die Verwendung von leeren Begriffen, wie falsch auch immer die zugrundeliegende Theorie und Überzeugung ist, die Kommunikation zwischen Personen erleichtert. So ist das Sprechen über Schmerzen in einem Diskurs mit einer anderen Person leichter im Vergleich zu einem Diskurs über die eliminierende physikalische Theorie und den Gehirnzustand XYZ, der in einer finalen Physik denjenigen Zustand herauspickt, welchen in der Alltagspsychologie zuvor vermeintlich, aber nicht tatsächlich, der Begriff "Schmerz" herauspickte. 
Ob nun ein Alb, Harry Potter, das Christkind, mindestens eine vermeintliche Gottheit oder der Nikolaus, wir sind uns ziemlich sicher, dass diese Dinge nicht tatsächlich existieren und keine Referenten von irgendwelchen Begriffen sind. Und per Analogie wird es sich bei Gültigkeit des Eliminativismus auch für mentale Begriffe herausstellen. Man denke nur daran, wenn dem Alb eine Absicht zugeschreiben wird, nämlich die Absicht, schlechte Träume hervorzurufen. Oder auch Harry Potter Gefühle zugeschrieben werden, nämlich die Angst, Lord Voldemort gegenüberstehen zu müssen. So unterliegen auch mentale Begriffe den kulturellen, pädagogisch-erzieherischen und pragmatischen Gründen, warum sie brauchbar im Alltag sind. An dieser Stelle sind wir an derselben Konklusion wie Corns angelangt, nur über eine andere Route. Das ist aber nicht alles, denn leere Begriffe sind nicht nur für den Alltag, sondern auch für die Wissenschaften brauchbar. Wenn leere Begriffe zusätzlich auch für den wissenschaftlichen Diskurs brauchbar sind, dann sind wir bei einem liberalen Eliminativismus angelangt, der auf ontologischer Ebene streng und traditionell ist, doch auf begrifflicher Ebene liberaler ist als der traditionelle und der wissenschaftliche Eliminativismus.

Warum leere Begriffe auch in den Wissenschaften einen Platz haben sollten, begründe ich durch die Brauchbarkeit negativer Existenzaussagen. Damit meine ich natürlich nicht eine Naturwissenschaft des Nicht-Existierenden, sondern wertvolle Einsichten durch das Benennen, was nicht der Fall ist. Beispielsweise wäre es eine naturwissenschaftlich bahnbrechende Erkenntnis, wenn die Nicht-Existenz von schwarzen Löchern bewiesen werden würde, in welchem gefinkelten Experiment auch immer. Solch ein klassisches Nullergebnis würde problemlos die hochrangigsten Journale füllen. Der Begriff "schwarzes Loch" würde keinen Referenten mehr haben und durch eine deskriptivistische Referenztheorie könnten wir es auch erklären, weil es nämlich nichts gibt, das der Beschreibung eines schwarzen Loches genügt. Blicken wir beim zweiten Beispiel in den neurowissenschaftlichen Bereich. Die Theorie einer "Großmutterzelle" besagt, dass es ein einzelnes Neuron gibt, das sehr komplexe und sehr spezifische Informationen speichert, wie eben das Abbild einer Großmutter. Wir haben aktuell gute Gründe anzunehmen, dass es gar keine Großmutterzellen gibt (Barwich, 2019), demnach ist auch der Begriff "Großmutterzelle" ohne jeglichen Referenten. Aber dennoch, es stellt einen Fortschritt dar, indem man zeigt, was es nicht gibt, damit zukünftige Forschung daraus lernen kann. Barwich argumentiert am Beispiel der Großmutterzelle so:

Science fails. It seems to fail at a high rate and with regularity... One may wonder why science is so successful despite such prevalent failures. The alternative is to suggest that it is because of them. (S. 1)

In the end, there are multiple ways of dealing with failures in science... Another, equally instructive option, outlined here, is to use them explicitely as 
failures to actively engage with our limits of understanding and probe for blind spots. (S. 10)

Zu guter Letzt möchte ich einräumen, dass die Verwendungshäufigkeit von leeren Begriffen in den Wissenschaften schwankt und durch bestimmte Forschungsrichtungen erhöht wird. Wenn es der Zeitgeist gebietet, einen vollständigen Übersichtsartikel über die Spezifität und Komplexität der Repräsentationseigenschaften von Neuronen zu sprechen, dann wird das Nennen von Großmutterzellen wohl auch vorkommen. Dann aber mit dem Hinweis, dass Großmutterzellen nicht existieren und auf diese Sackgasse aufbauend die neuere und wahrscheinlichere Theorie entwickelt wurde, wie auch immer diese neue Theorie aussieht. Wenn wir diese Einsicht in den mentalen Bereich übertragen, dann können auch verschiedenste qualitative und intentionale Zustände, obwohl die zugrundeliegende Theorie falsch ist und die jeweiligen mentalen Begriffe leer sind, eine gewichtige Rolle in der Entwicklung einer finalen Physik spielen. Die Rolle ist die, durch das Versagen der Alltagspsychologie eine bessere Theorie über unser menschliches Erleben und Handeln vorzubereiten. So wie es auch der wissenschaftliche Fortschritt in den anderen Bereich zeigt.

\subsubsection{Fiktionalismus für den liberalen Eliminativisten}

Die vorigen Analysen zur Brauchbarkeit von leeren Begriffen stellten das erste von zwei Problemen dar, die Corns aufgeworfen hat. Das zweite Problem war, was es genau bedeutet, wenn etwas wirklich existiert, oder nicht wirklich existiert und wie es möglich ist, dass wir uns problemlos auf nicht-existierende Dinge wie den Alb, Harry Potter und verschiedenste mentale Entitäten beziehen. Das Problem für den Eliminativisten, ob nun von wissenschaftlicher oder liberaler Spielart, ist der folgende scheinbare Widerspruch: Einerseits sind leere Begriffe ohne jeglichen Referenten, aber andererseits werden die leeren Begriffe im Alltag und gegebenenfalls in den Wissenschaften weiterverwendet und scheinen sich dennoch auf etwas zu beziehen. In der weiteren Diskussion werde ich oftmals ausschließlich den Begriff "Eliminativismus" verwenden. Damit meine ich den liberalen Eliminativismus, den ich in diesem Kapitel verteidige, außer es erfolgt eine nähere Spezifizierung der eliminativistischen Spielart.

Meine These ist, dass die Auflösung dieses Widerspruches durch die Akzeptanz eines Fiktionalismus passieren kann (Daly, 2013; Wallace, 2016). Ein Fiktionalist zu sein bedeutet, so zu tun, als ob die Fiktion Wahrheit wäre, und vorzugeben, dass es der Fall sei, wie es in der Fiktion dargelegt wird. Das trifft die Romanreihe zu Harry Potter genauso wie den mentalen Bereich. Wir können im Alltag in Diskussion treten über Geschichten und Filme, uns austauschen, wie Harry Potter Lord Voldemort schneller besiegen hätte können und mit unseren Kindern sprechen, ob sie vorm großen, bösen Wolf genauso viel Angst gehabt hätten wie Rotkäppchen. Während wir darüber sprechen, wissen wir genau, dass es eine Fiktion ist, 
nichtsdestotrotz tun wir es, weil wir Vorteile darin sehen. Ähnlich ist es auch im wissenschaftlichen Bereich, wenn wir von der Nicht-Existenz von schwarzen Löchern sprechen würden. Wir würden in Diskussionen die Charakteristika von schwarzen Löchern näher erörtern, welche Eigenschaften sie hätten, wie sie die Raumzeit biegen würden, wo der Ereignishorizont wäre und dergleichen. Jedes Gespräch in diesen Bereichen setzt eine implizite Präambel voraus, dass das Gesagte nur dann gilt, falls auch Harry Potter, Grimms Märchen, die zugrundeliegende Theorie über schwarze Löcher und dergleichen als wahr angenommen werden würden. Wenn man so will, redet man über mögliche Welten, die ziemlich verschieden zu unserer aktualen Welt sind. Gespräche über mögliche Welten scheinen breiter akzeptiert zu sein, doch ist es nichts anderes als ein Gespräch über etwas Fiktives.

In gleicher Weise verhält es sich bei einem mentalen Fiktionalismus. Wir reden im Alltag über Schmerzen, Liebe und Hass, über unsere Wünsche, Absichten und Überzeugungen. Dabei beziehen wir uns auf die Alltagspsychologie, die sich zu Zeiten einer finalen Physik als falsch herausstellt. Alle postulierten Entitäten der Alltagspsychologie werden ontologisch eliminiert, aber trotzdem würden wir über unser vermeintlich mentales Leben weiter sprechen. Die fehlende Referenz in der aktualen Welt steht nicht im Widerspruch zu einer vorhandenen Referenz innerhalb einer Fiktion. Innerhalb einer angenommenen Gültigkeit der Alltagspsychologie, würde es sich so-und-so ergeben, wie unser mentales Leben zusammenwirkt, um unser Erleben und Verhalten zu erklären und vorauszusagen. In gleicher Art und Weise würde es den Wissenschaftlern gehen. Sie könnten in einer goldenen Zeit der Physik immer noch von mentalen Entitäten sprechen, beispielsweise in Vorträgen, auf Kongressen und in geschichtlichen Abrissen der Psychologie. Sie würden sagen, dass Schmerzen, Liebe und Hass sowie Wünsche, Absichten und Überzeugungen nicht existieren, so wie es einst die Alltagspsychologie postulierte. Doch man könnte näher auf die Falschheit der Alltagspsychologie eingehen und beschreiben, dass man an die Privatheit und absolute Subjektivität des Mentalen glaubte, dass die Erkenntnis des Mentalen unkorrigierbar und unmittelbar gewesen sei. Wie auch in den Bereichen zuvor, würde jedes Gespräch in diesen Bereichen die implizite Präambel voraussetzen, dass das Gesagte nur dann gilt, falls auch die zugrundeliegende Alltagspsychologie als wahr angenommen werden würde.

Damit hat der liberale Eliminativist beides erreicht: Er eliminiert das Mentale auf der ontologischen Ebene, kann jedoch als Fiktionalist dennoch über Mentales sprechen. Warum will ein Eliminativist über Mentales sprechen? Die überzeugendsten Gründe sehe ich im Alltag auf der Basis kultureller, pädagogisch-erzieherischer und pragmatischer Überlegungen. In den Wissenschaften sehe ich den Grund vorrangig im Tätigen von negativen Existenzaussagen, auf dessen Basis ein weiterer Fortschritt geschehen kann. 


\subsection{Wie einfach und sparsam ist der liberale A-priori-Eliminativismus?}

Bevor wir zur Vergleichbarkeit des liberalen Eliminativismus mit der Typen-Identitätstheorie a priori kommen, müssen wir noch drei Ergänzungen treffen. Wir wissen aus Kapitel 4, dass eine adäquate physikalistische Bewusstseinstheorie eine sein soll, die a priori gilt und die analytisch wahr ist. Der Feststellung, ob der liberale Eliminativismus wahr ist, kann ohne Rückgriff auf Erfahrung getätigt werden: Das ist die A-priori-Anforderung. Darüber hinaus wissen wir es deshalb a priori, weil der liberale Eliminativismus aufgrund der involvierten Wörter wahr ist: Das ist die Analytizitäts-Anforderung. Gleichzeitig ist auch hier der Eliminativismus eine kontingente Wahrheit, weil es darauf ankommt, wie unsere aktuale Welt beschaffen ist. Schließlich muss sich jede gangbare physikalistische Bewusstseinstheorie mit Typen beschäftigen und nicht bloß mit einzelnen Vorkommnissen: Das ist die Typen-Anforderung. Eine Argumentation, die in ähnlicher Form bei der Identitätstheorie am Werk war, ist auch hier mutatis mutandis anwendbar. Nehmen wir als Ausgangspunkt die Kernforderung des Eliminativismus her:

(E) Genau dann, wenn alle mentalen Begriffe leer sind, werden alle mentalen Typen durch physikalische Typen ersetzt und alle mentalen Begriffe werden durch physikalische Begriffe ersetzt.

Die A-priori-Anforderung wird dadurch erfüllt, indem $(E)$ so zu deuten ist, dass kein Blick in die Welt geworfen werden muss, um zu wissen, dass das Physikalische das Mentale ersetzt. Alles, was es braucht, ist ein hinreichend klares Verständnis des Physikalischen. Durch die fehlende Deckungsgleichheit des Physikalischen mit dem Mentalen aufgrund einer fehlenden Referenz der mentalen Begriffe kommt das Ersetzen des Mentalen durch das Physikalische ohne weiteres Zutun und notwendigerweise hinzu. Das Ersetzen geschieht sowohl auf ontologischer Ebene als auch auf begrifflicher Ebene. Die Analytizitäts-Anforderung wird dadurch erfüllt, indem das hinreichende Verständnis des Physikalischen zur Wahrheit des Physikalismus führt. Die Typen-Anforderung wird dadurch erfüllt, indem ein Dualismus durch die Hintertüre vermieden wird und auf eine eliminativistische Position verzichtet wird, die sich ausschließlich auf Tokens bezieht. Denn falls lediglich auf Token-Ebene alles eliminiert wird, sagt das noch nichts darüber aus, ob auch auf Typenebene alles Mentale eliminiert werden kann. Ein Token-Eliminativist wäre somit mit einem Dualismus auf Typenebene vereinbar.

Der Titel dieses Kapitels ist, dass der Eliminativismus ein stiller Begleiter sei. Um dies zu rechtfertigen, muss der Eliminativismus, so wie ich inn im Sinne eines liberalen TypenEliminativismus a priori herausgearbeitet habe, mit der Typen-Identitätstheorie a priori verglichen werden. Hierfür muss auch derselbe Maßstab angewandt werden, der im Vergleich der Identitätstheorie mit den restlichen vorgestellten Bewusstseinstheorien am Werk war, nämlich die Kriterien der Einfachheit und Sparsamkeit. 
Um das Kriterium der Einfachheit anzuwenden, möchte ich nochmals auf die grundlegenden Unterschiede der beiden Theorien hinweisen. Obwohl sowohl der Identitätstheoretiker als auch der Eliminativist eine bestimmte Entwicklung der Alltagspsychologie annimmt, ist die genaue Erwartung der Entwicklungsrichtung diametral auseinandergehend. Der Erstere ist überzeugt, dass beide Bereiche hinreichend deckungsgleich sind, sodass eine Reduktion geschehen kann. Der Zweitere ist überzeugt, dass beide Bereiche in keinster Weise deckungsgleich sind, sodass der mentale Bereich komplett durch den physikalischen Bereich ersetzt wird. Genau diese Unterschiede bergen auch verschiedene Konsequenzen, denn bei Gültigkeit des Eliminativismus muss keine Supervenienz zu einer Superdupervenienz werden, keine Typenidentität verteidigt werden und keine multiple Realisierbarkeit widerlegt werden. Ebenso stellt sich die Frage nach einer A-priori-Ableitbarkeit des Mentalen, und gleichzeitig die einer A-posteriori-Ableitbarkeit, gar nicht mehr, genauso wenig wie die Frage nach der Übersetzbarkeit des mentalen Vokabulars in das physikalische Vokabular. Wenn nun alle diese viel diskutierten Themenbereiche beim Eliminativismus wegfallen, dann ist er in dieser Hinsicht einfacher als jegliche Identitätstheorie. Am Beispiel der vier Deskriptoren der Einfachheit, die ich im letzten Kapitel aufgestellt habe, ist der Eliminativismus deshalb einfacher, weil er kognitiv zugänglicher, rascher verstehbar, übersichtlicher dargestellt und eindeutiger erfassbar ist. Denn was ist einfacher, als lediglich etwas von der ontologischen und begrifflichen Bildfläche zu eliminieren? Die begriffliche Elimination betrifft in diesem Kontext die tatsächlich vorhandene, aktuale Welt. Wie zuvor ausgeführt, bleibt die Verwendungsmöglichkeit der eliminierten Begriffe innerhalb einer Fiktion natürlich bestehen.

Hinsichtlich der Sparsamkeit möchte ich als Erstes die ontologische Sparsamkeit betrachten. Hier ergibt sich kein Unterschied zu irgendeiner anderen reduktiven Bewusstseinstheorie, denn alles, was existiert, ist physikalisch. Es ergibt sich jedoch ein Unterschied bei der begrifflichen Sparsamkeit. Im Alltag und im wissenschaftlichen Diskurs in der Ära einer finalen Physik wird das mentale Vokabular durch das physikalische Vokabular ersetzt, obgleich sich in einigen wenigen konkreten Anwendungsbereichen die Weiterverwendung des bereits eliminierten Vokabulars als brauchbar erweisen kann. Diese Brauchbarkeit spielt sich in den Wissenschaften in den zuvor erwähnten engen Grenzen von negativen Existenzaussagen und sich daraus entwickelnden neuen Ideen bis hin zu einer finalen Physik ab. Beim Erreichen der Ära einer finalen Physik ist die Verwendung eines mentalen Vokabulars beschränkt auf historische Andeutungen und das Feststellen, was sich in der Geschichte der Physik als nicht-existierend herausgestellt hat. Die Brauchbarkeit ist deshalb auf historische negative Existenzaussagen beschränkt, weil eine vollendete Physik das Endstadium der Naturwissenschaften ist und alles Physikalische erschöpfend erfasst wurde. Ähnlich verhält es sich auch im alltäglichen Diskurs, bei dem keine strikte Notwendigkeit eines mentalen Vokabulars besteht, aber diese alltagspsychologischen bzw. mentalen Begriffe wohl im Sinne 
einer Fiktion, beispielsweise in Gedichten, Romanen und ähnlichen Textgattungen, einen weiteren Gebrauch finden. Es geht nicht darum, den Gebrauch des mentalen Vokabulars zu verbieten, sondern darum, lediglich zu zeigen, dass deren Verwendung keine strikte Notwendigkeit mehr besitzt, sondern lediglich eine Brauchbarkeit in einem eng umgrenzten Anwendungsbereich besitzt.

Hingegen sind bei einer Übersetzung des mentalen in das physikalische Vokabular noch beide Begriffsgattungen vorhanden, da ein entsprechendes Begriffspaar wie beispielsweise "Furchterlebnis = Aktivierung der neuronalen Struktur N" synonym ist. Eine begriffliche Elimination ist stärker als eine begriffliche Reduktion, weil der Eliminativist den physikalischen Begrifflichkeiten nicht aufgrund der Synonymität von Begriffen den Vorzug geben muss, sondern er den physikalischen Begrifflichkeiten den Vorzug aufgrund des Fehlens einer anderen Alternative gibt. Wenn eines durch das andere nun komplett ersetzt wird, sinkt auch die Wahrscheinlichkeit, das ersetzte Vokabular, zu welchem Zwecke auch immer, wieder in den Sprachgebrauch aufzunehmen, außer im Sinne einer Fiktion. Deshalb ist der Eliminativismus schlussendlich begrifflich sparsamer als eine Identitätstheorie.

Aber warum sollte dann nicht dem Eliminativismus der Vorzug gegeben werden? Meine Antwort ist, dass der Eliminativismus und die Identitätstheorie auf gleicher Evidenzstufe sind, nämlich dahingehend, dass die Evidenzlage noch sehr unklar ist. Es ist noch nicht vorherzusehen, in welche Richtung die Entscheidung im Lichte der zukünftigen Erkenntnisse gehen wird. Es wird erst bei Vorliegen einer finalen Altagspsychologie deutlich, ob eine Entsprechung zu einer finalen Physik vorliegt, kontra Eliminativismus, oder keine Entsprechung vorliegt, kontra Identitätstheorie. Aus diesem Grunde sehe ich beide Ansätze noch im Rennen und möchte keiner Position aus theoretischen Überlegungen zur Einfachheit und zur Sparsamkeit den Vorzug geben.

\subsection{Die Definition des Physikalismus}

Am Ende dieses Kapitels ist es nun möglich, eine Definition des Physikalismus vorzulegen. Wir wissen, was "physikalisch" bedeutet, und wir wissen, wie die Relation zwischen dem Mentalen und dem Physikalischen zu bestimmen ist. Zuerst möchte ich die Relation zwischen dem Mentalen und dem Physikalischen abschließend explizieren, um darauffolgend die abschließende Definition des Physikalismus vorzulegen.

\subsubsection{Das Mentale und Physikalische: eine Definition deren Relation Teil 2} Als Konklusion bleibt übrig, dass entweder eine Typen-Identitätstheorie a priori wahr ist oder der Eliminativismus wahr ist. Der Eliminativismus lässt sich nicht a priori ausschließen, sondern stellt womöglich eine Konsequenz einer finalen physikalischen Theorie dar und ist abhängig davon, 
welches Schicksal der Alltagspsychologie widerfahren wird. Da das Schicksal der Alltagspsychologie nicht gewiss ist und wir den zukünftigen Ausgang nicht klar feststellen können, bleiben beide physikalistischen Theorien als Optionen erhalten. Wir haben auch gesehen, dass sich die Identitätstheorie und der Eliminativismus nicht gegenseitig ausschließen, sondern vielmehr ergänzend wirken. Nämlich da, wo keine Reduktion möglich ist, ist eine Elimination möglich und umgekehrt. Eine fehlende Ableitung des Mentalen aus dem Physikalischen bedeutet in diesem System nicht notwendigerweise, dass der Physikalismus als Gesamtes falsch ist, sondern dass die infrage stehende mentale Entität einfach auch eliminiert werden kann. In welche Richtung das Pendel zwischen den Endpunkten "Reduktion" und "Elimination" ausschlägt, bleibt eine empirische Frage, genauso wie die Frage, wie unsere Welt tatsächlich beschaffen ist und die These "Reduktion oder Elimination" sich als wahr oder falsch herausstellt.

Der erste Anlauf aus dem vorigen Kapitel, die Relation zwischen dem Mentalen und dem Physikalischen zu bestimmen, möchte ich jetzt noch weiter verfeinern, indem ich die Relation disjunktiv um den liberalen Eliminativismus erweitere. Die "Oder"-Verknüpfung ist aus diesem Grunde als ein einschließendes Oder zu verstehen und keinesfalls als ein gegenseitig ausschließendes Oder. Unsere Analysen in diesem Kapitel 5, zusammen mit den Erkenntnissen aus den Kapiteln 3 und 4, führen uns zur nachstehenden Definition der Relation zwischen dem Mentalen und Physikalischen.

(R2) Eine mentale Entität ist genau dann nichts anderes als eine physikalische Entität, wenn kontingenterweise entweder a priori und analytisch gilt, dass jeder Typus einer mentalen Entität

a) mit i) dem Typus einer physikalischen Basisentität oder mit ii) dem Typus einer physikalischen Entität höherer Ordnung, die in einer bestimmten Relation zu den physikalischen Basisentitäten steht, ontologisch identisch ist,

und dass jeder Begriff einer mentalen Entität

b) mit dem Begriff i) einer physikalischen Basisentität oder mit ii) dem Begriff einer physikalischen Entität höherer Ordnung, die in einer bestimmten Relation zu den physikalischen Basisentitäten steht, identisch und damit synonym ist, oder a priori und analytisch gilt, dass jeder Typus einer mentalen Entität

c) mit i) dem Typus einer physikalischen Basisentität oder mit ii) dem Typus einer physikalischen Entität höherer Ordnung, die in einer bestimmten Relation zu den physikalischen Basisentitäten steht, ersetzt und dadurch eliminiert wird,

und dass jeder Begriff einer mentalen Entität 
d) mit i) dem Begriff einer physikalischen Basisentität oder mit ii) dem Begriff einer physikalischen Entität höherer Ordnung, die in einer bestimmten Relation zu den physikalischen Basisentitäten steht, ersetzt und dadurch eliminiert wird.

In dieser Definition sind alle Erkenntnisse der letzten drei Kapitel miteingeflossen. Es ist entweder der Fall, dass eine Typen-Identität zwischen dem Mentalen und Physikalischen gegeben ist und mentale Begriffe mit physikalischen Begriffen bedeutungsgleich sind, oder das Mentale wird auf ontologischer und begrifflicher Ebene durch das Physikalische ersetzt. In beiden Fällen handelt es sich um eine Relation, die a priori und analytisch gilt. Ebenso ist die Identität zwischen dem Physikalischen und dem Mentalen kontingent, weil alles Physikalische von der Beschaffenheit unserer aktualen Welt abhängt, und diese hätte auch anders sein können.

\subsubsection{Der Physikalismus expliziert}

Die ursprünglichen, intuitiven Definitionen des Physikalismus, die den Ausgangspunkt der Analyse darstellten, waren die folgenden beiden:

(P1a) Alles Existierende ist nichts anderes als etwas Physikalisches.

(P1b) Alles ist physikalisch.

Um in eine fruchtende Diskussion innerhalb der Philosophie des Geistes zu kommen, muss zuerst festgelegt werden, was konkret unter dem Physikalismus verstanden wird. Die vorigen Analysen lassen nun eine genaue Definition der These des Physikalismus zu, in der vor allem die Definition des Physikalischen aus Kapitel 2 und die Definition des Ausdruckes "nichts anderes als" aus den Kapiteln 3 bis 5 in eine gemeinsame Darlegung des Physikalismus zusammengeführt werden:

(P1c) Der Physikalismus ist die kontingente These, dass a priori und analytisch gilt, dass in einer finalen Theorie über Ursachen und Wirkungen der objektiven/subjektunabhängigen Welt jeder Typus einer mentalen Entität

a) mit i) dem Typus einer Basisentität oder mit ii) dem Typus einer Entität höherer Ordnung, die in einer bestimmten Relation zu den Basisentitäten steht, ontologisch identisch ist, und dass jeder Begriff einer mentalen Entität 
b) mit dem Begriff i) einer Basisentität oder mit ii) dem Begriff einer Entität höherer Ordnung, die in einer bestimmten Relation zu den Basisentitäten steht, identisch und damit synonym ist,

oder dass a priori und analytisch gilt, dass in einer finalen Theorie über Ursachen und Wirkungen der objektiven/subjektunabhängigen Welt jeder Typus einer mentalen Entität

c) mit i) dem Typus einer Basisentität oder mit ii) dem Typus einer Entität höherer Ordnung, die in einer bestimmten Relation zu den Basisentitäten steht, ersetzt und dadurch eliminiert wird,

und dass jeder Begriff einer mentalen Entität

d) mit i) dem Begriff einer Basisentität oder mit ii) dem Begriff einer Entität höherer Ordnung, die in einer bestimmten Relation zu den Basisentitäten steht, ersetzt und dadurch eliminiert wird.

Diese These des Physikalismus ist auf Basis der vorhergehenden Kapiteln so zu verstehen, dass falls der Physikalismus wahr ist, dann ist die obige Explikation des Physikalismus wahr. So wurde insbesondere in den Kapiteln 3 bis 5 argumentiert, dass (P1c) die beste Version einer physikalistischen Position ist im Vergleich zu allen anderen physikalistischen Positionen. Entweder ist eine Typen-Identität zwischen dem Mentalen und Physikalischen gegeben und mentale Begriffe sind synonym mit physikalischen Begriffen, oder das Mentale wird auf ontologischer und begrifflicher Ebene durch das Physikalische ersetzt und dadurch eliminiert. Als Basisentitäten einerseits und als Entitäten höherer Ordnung andererseits, die in einer bestimmten Relation zu den Basisentitäten stehen, stehen diejenigen Entitäten zur Verfügung, die in einer finalen physikalischen Theorie postuliert werden. Deshalb wird das Auslangen mit den Begriffen "Basisentität" und "Entität höherer Ordnung" gefunden, ohne explizit "physikalische Basisentitäten" oder "physikalische Entität höherer Ordnung" zu schreiben. Darüber hinaus wird der Begriff "physikalische Theorie" umgangen und mit einer "Theorie über Ursachen und Wirkungen der objektiven/subjektunabhängigen Welt" umschrieben. Die These des Physikalismus ist eine Wahrheit, die a priori und analytisch gilt, aber nichtsdestotrotz kontingent wahr ist. Die Kontingenz kommt daher, weil eine finale physikalische Theorie und deren Postulate davon abhängt, wie unsere aktuale Welt beschaffen ist. Die Explikation des Physikalismus ist als erster Schritt wichtig, um daraufhin zu prüfen, ob der Physikalismus auch tatsächlich wahr ist. 


\subsection{Zusammenfassung}

Dieses Kapitel hat damit begonnen, zwei Einwände aus der Literatur und einen Einwand im Kontext dieser Arbeit gegen den Eliminativismus zu diskutieren. Nach Entschärfung dieser Einwände habe ich die Altagspsychologie dargelegt und erarbeitet, dass Elimination und Reduktion sich gegenseitig ergänzen. Wie das Schicksal der Alltagspsychologie aussieht, bleibt eine offene Frage und hängt davon ab, inwieweit eine Entsprechung zwischen ihr und einer finalen Physik aussehen wird. Weiters habe ich die Voraussetzung einer deskriptivistischen Referenztheorie diskutiert und gezeigt, wie ein Deskriptivismus durch Einschränkung der Referenz auf die aktuale Welt, und allenfalls auf hinreichend ähnliche Welten, verteidigt werden kann. Ich habe in diesem Kapitel einen liberalen Eliminativismus entwickelt, der durch die Akzeptanz eines Fiktionalismus beides hat, sowohl die Brauchbarkeit leerer Begriffe im Alltag sowie in den Wissenschaften als auch die ontologische Elimination des vermeintlichen Referenten. Im Vergleich zur Typen-Identitätstheorie a priori zeigt sich der liberale Eliminativismus a priori als genauso ontologisch sparsam, jedoch begrifflich sparsamer und einfacher. Ich habe diesen Vergleich mit der Einsicht geschlossen, dass unter allen physikalistischen Bewusstseinstheorien bei Anwendung der Einfachheit und Sparsamkeit auch noch der Eliminativismus im Rennen ist und er die zweite Möglichkeit neben einer Identität zwischen dem Mentalen und Physikalischen als gangbare Option darstellt. Dabei sehe ich die Identitätstheorie und den Eliminativismus nicht als einander ausschließend, sondern als sich gegenseitig ergänzend, abhängig von der tatsächlichen Entwicklung der Alltagspsychologie in einer Ära der finalen Physik. Da die Entwicklung der Alltagspsychologie noch nicht abgeschlossen ist, kann vorweg keine Entscheidung zugunsten des Eliminativismus getroffen werden, trotz seiner theoretischen Vorzüge in Bezug auf die begriffliche Sparsamkeit und die Einfachheit verglichen mit der Identitätstheorie. Abschließend habe ich meine Definition des Physikalismus dargelegt, die alle Erkenntnisse aus den vorangehenden Kapiteln verwertet. 
So, so you think you can tell Heaven from hell?

Blue skies from pain?

Can you tell a green field

From a cold steel rail?

A smile from a veil?

Do you think you can tell?

Pink Floyd (1975) 


\section{Dualismus vs. Physikalismus}

Das erste Ziel dieses Kapitels ist es, die überzeugendste dualistische Position in der Philosophie des Geistes als Gegenposition zum Physikalismus zu identifizieren. Dabei werde ich zum einen zwischen einem Substanzdualismus sowie einem Eigenschaftsdualismus und zum anderen zwischen einem interaktionistischen sowie einem epiphänomenalistischen Dualismus unterscheiden. Im Zuge dieser Diskussion identifiziere ich einen epiphänomenalistischen Eigenschaftsdualismus als die überzeugendste Position in den Händen des Dualisten. Als zweites Ziel stelle ich die einflussreichsten antiphysikalistischen Argumente vor, nämlich das Argument des unvollständigen Wissens, das Erklärungslückenargument, das Fledermausargument, das Zombie-Argument und das Argument vom Begreifen phänomenaler Eigenschaften. Anschließend führe ich als drittes Ziel die einflussreichsten Argumente für den Physikalismus an und bereite so die Dialektik zwischen den rivalisierenden Positionen vor. Unter diese einflussreichsten Argumente fallen diejenigen, welche die Identität als beste und einfachste Erklärung sehen oder als Konklusion eines deduktiven Arguments oder als Konsequenz der kausalen Geschlossenheit oder als wahrscheinlichste Erklärung oder als anzunehmende Standardsicht.

\subsection{Das gewisse Extra}

Alle dualistischen Positionen teilen sich die Voraussetzung, dass neben allen physikalischen Entitäten noch etwas Mentales existiert. Wie dieses mentale Extra im Konkreten zu verstehen ist, variiert ja nach vertretener dualistischer Position. Eine Einteilung der dualistischen Positionen kann vielfältig erfolgen, so kann beispielsweise ein Substanzdualismus vertreten werden oder lediglich ein Eigenschaftsdualismus. Darüber hinaus kann hinsichtlich der kausalen Beziehungen zwischen dem Physikalischen und dem Mentalen entweder ein interaktionistischer Dualismus oder ein nicht-interaktionistischer Dualismus unterschieden werden. Diese beiden Beschreibungsdimensionen des Dualismus sind die häufigsten und tragen letztendlich zur Plausibilität des Dualismus bei und dazu, welche genaue Spielart des Dualismus zusammengenommen als die überzeugendste angenommen werden kann.

Als eigenschaftsdualistische Position genießt der Epiphänomenalismus große Bedeutung, der mentalen Eigenschaften keine kausale Rolle zuschreibt (z.B. Chalmers, 1996). Hingegen ist ein interaktionistischer Dualismus oftmals mit dem Substanzdualismus verbunden, obgleich auch Vertreter eines interaktionistischen Eigenschaftsdualismus ihre Argumente vorgebracht haben (z.B. Popper, 1994). Der interaktionistische Dualismus vertritt die These, dass das Mentale auf das Physikalische als essentiell verschiedene Entität kausal einwirken kann. 


\subsubsection{Der Substanz- und der Eigenschaftsdualismus}

Auf der Seite des Dualismus befindet sich der klassische Substanzdualismus nach Descartes $(1641)^{48}$, der in mehr oder weniger starken Veränderungen in der zeitgenössischen Literatur vor allem von Swinburne (1986, 2019), Hart (1988), Eccles (1989) und Foster (1991) vertreten wird. Das, was Descartes damals erreichen wollte, wird im Wesentlichen auch noch von gegenwärtigen Vertretern gewollt und betrifft das Mentale als etwas real Existierendes in der stärksten möglichen Form. Ich möchte mich deshalb auf Descartes' Beweggründe konzentrieren, der im Allgemeinen nach Gewissheit strebt, so auch in der Diskussion des Körper-Geist-Problems. In diesem Streben will er zumindest eine unerschütterliche Sache feststellen: "... bis ich etwas Gewisses erkenne, sollte es auch nur die Gewissheit sein, dass es nichts Gewisses gibt" (S. 77, Kap. 1). Er führt den Begriff eines Betrügers ein, der ständig seine Gedanken beeinflusst und der ihn mit Absicht täuscht. Demnach ist nichts gewiss, weil ja bloße Täuschung vorliegt. Somit kann man nur an allen Dingen zweifeln. Descartes will darauf hinaus, dass zwar alle Körper angezweifelt werden können, doch der Umstand, dass ich zweifle, kann nicht bezweifelt werden und somit existiere ich. Dieses Urteil ist nun notwendigerweise wahr und Descartes hat seine Gewissheit erreicht, und zwar in der Hinsicht, dass das Denken das Ausschlaggebende ist. Nur dies lässt er gelten und schließt, dass "... ich genaugenommen lediglich ein denkendes Ding [bin]" (S. 83, Kap. 6). Aus der Tatsache, dass ich etwas sehe, höre, fühle und Ähnliches tue, folgt, dass ich bin. Durch mein Bewusstsein dessen, was ich betrachte, folgt meine Existenz und diese Existenz ist für Descartes das denkende Ding.

Unter einem Körper versteht Descartes etwas, das räumlich begrenzt ist, räumlich umschrieben werden kann und durch unsere Sinne wahrgenommen werden kann. Er prägt dafür den Begriff res extensa (obwohl er in der Zweiten Meditation nie diesen Begriff verwendet, sondern bloß corporis). Weiters sind Körper etwas Ausgedehntes, Biegsames und Veränderliches. Er macht diesen Punkt unter anderem anhand einer Kerze klar, die aus Wachs besteht und nach dem Kontakt mit Feuer zerfließt, sich ausdehnt, heiß wird und dergleichen. Dem gegenüber steht der Geist oder die Seele oder der Verstand oder die Vernunft. Descartes führt dazu den Begriff res cogitans ein. Er charakterisiert es als ein Ding, "... das zweifelt, einsieht, bejaht, verneint, will, nicht will, das auch bildlich vorstellt und empfindet" (S. 87, Kap. 8). Somit schreibt er dieser res cogitans alle mentalen Eigenschaften zu.

Der cartesische Dualismus nimmt an, dass Körper und Geist zwei Substanzen darstellen, die sich gegenseitig ausschließen. Beide Substanzen werden jeweils durch notwendige Eigenschaften definiert, sodass eine notwendige Eigenschaft für die eine Substanz nicht auch zugleich eine notwendige Eigenschaft für die andere Substanz sein kann. Denn entweder ist es etwas Physikalisches, dann kommt dieser Substanz die spezifische Eigenschaft "ausgedehnt zu sein" zu, oder es ist etwas Mentales, dann kommt dieser Substanz die spezifische Eigenschaft

${ }^{48}$ Alle folgenden Seitenangaben beziehen sich auf die Ausgabe von 1986, herausgegeben von G. Schmidt. 
"denkend zu sein" zu. Beide Substanzen schließen sich deshalb einander aus, weil dem Physikalischen nie die Eigenschaft zukommt, denkend zu sein, und gleichsam dem Mentalen nie die Eigenschaft zugeschrieben werden kann, ausgedehnt zu sein. Dieses cartesische Erbe der jeweils verschiedenen Eigenschaftszuschreibung des Physikalischen und des Mentalen ist uns bereits im zweiten Kapitel begegnet, ebenso die Antwort der modernen Naturwissenschaft, dass es sich doch nicht so einfach darstellt. Man denke an Photonen, die masselos und nicht ausgedehnt sind, und trotzdem Teil der physikalischen Ontologie sind. Darüber hinaus wird durch diesen Standpunkt klar, dass eine physikalische Substanz nur physikalische

Eigenschaften aufweist und eine mentale Substanz nur mentale Eigenschaften. Dieser Punkt ist deshalb relevant, weil der Substanzdualismus die stärkste Form eines Dualismus darstellt und in einer bestimmten logischen Beziehung zum Eigenschaftsdualismus steht. Diese Beziehung ist derart, dass aus einem Substanzdualismus ein Eigenschaftsdualismus folgt, aber nicht umgekehrt.

Es ist im Laufe der Zeit in den Reihen der Dualisten Zweifel aufgetreten, ob solch eine starke Form des Dualismus auch tatsächlich notwendig ist. Ein Punkt in der Diskussion war eine gewisse Abschwächung eines Substanzdualismus und trotz alledem das Wahren des Mentalen als etwas Eigenständiges und Echtes. Daraus hat sich der Eigenschaftsdualismus entwickelt. Falls in der gegenwärtigen Literatur ein Dualismus vertreten wird, dann ist es vor allem diese Form des Dualismus (z.B. Chalmers, 1996). Indem man sich ontologisch lediglich auf zwei verschiedene Arten von Eigenschaften verpflichtet und nicht auf zwei verschiedene Arten von Substanzen, ist der Eigenschaftsdualismus zwar die logisch schwächere Form, doch gleichsam die am meisten akzeptierte dualistische Position. Hier existiert eine Art von Substanz, nämlich die physikalische, die eben zweierlei Klassen von Eigenschaften aufweist. Ob nun nach Abwägen der Vor- und Nachteile der Eigenschaftsdualismus tatsächlich deutlich besser ist als der Substanzdualismus, wird von einigen infrage gestellt. So schließt Lycan (2013, S. 541) nach einem systematischen Vergleich: "Verdict: PD [property dualism] is perhaps a little better off than SD [substance dualism]. But not so much better off that property dualists should go on boasting that they are not so crazy as to be Cartesians".

\subsubsection{Der interaktionistische und der epiphänomenale Dualismus}

Weil wir den Wunsch nach etwas Süßem haben, holen wir uns eine Tafel Schokolade. Und wir geben einen schmerzerfüllten Schrei von uns, weil wir uns das Bein auf dem Weg zur Süßigkeitenlade angestoßen haben. Unsere alltagspsychologischen Annahmen sagen uns, dass unser mentales Leben kausal wirksam ist. Der interaktionistische Dualismus, sei es nun in der Spielart eines Eigenschafts- oder eines Substanzdualismus, sagt genau dasselbe: Das Mentale als etwas real Existierendes und Nicht-Reduzierbares beeinflusst unsere Welt kausal. Doch wie beeinflussen sich zwei einander ausschließende Dinge? Dies stellt das größte 
Problem eines Interaktionismus dar, doch Dualisten haben Lösungsvorschläge erarbeitet. So sieht Popper (1994) den Interaktionismus als ein Ergebnis der Evolution und für Descartes (1649; sechste Meditation in 1641) findet die Körper-Geist-Interaktion in der Zirbeldrüse statt. Der große Vorteil des interaktionistischen Dualismus ist die Tatsache, dass er unseren altäglichen Erfahrungen entspricht und unsere Grunderwartung unterstreicht, wie das gemeinsame Auftreten eines Wunsches oder eines Gefühls mit bestimmten Verhaltensweisen und beobachtbaren Vorkommnissen zusammenhängt. Es hängt deshalb zusammen, weil beide Existenzbereiche schlichtweg miteinander in beiden Richtungen interagieren.

Wären da nicht Gegner, welche die Probleme der mentalen Kausalität als schwerwiegender einschätzen als sie die Vorzüge einer Lösung entlang unserer Intuitionen und Alltagserfahrungen hochleben lassen. Als Konkurrenzposition zum Interaktionismus ist der Epiphänomenalismus zu nennen, den Huxley (1874) in die Diskussion miteingebracht hat. Wenn man einen Epiphänomenalismus vertritt, dann vertritt man die These, dass alles Mentale wirkungslos ist und damit auch nicht in der physikalischen Welt kausal wirksam sein kann. Das Mentale sollte nach Huxley lediglich als der Rauch einer Lokomotive angesehen werden, der durch die Verbrennung von Kohle entsteht, aber selbst in keinster Weise Einfluss darauf nimmt, ob die Lokomotive fährt. Der große Vorteil des Epiphänomenalismus ist, dass er die konkreten einzelnen Schritte, wie zwei wesensverschiedene Dinge untereinander kausal wirksam sind, nicht explizieren muss. Wie auch beim Interaktionismus kann ein Epiphänomenalismus prinzipiell in einer substanz- oder einer eigenschaftsdualistischen Spielart vertreten werden. Man kann in gewisser Weise Malebranche (1997) als einen epiphänomenalistischen

Substanzdualisten verstehen, weil eine vermeintliche Kausalität zwischen dem Mentalen und dem Physikalischen eigentlich durch Gott verursacht wird. Wir Menschen hätten bloß den Schein, die Illusion, dass das Mentale kausal wirksam wäre, aber eigentlich war es Gott. Was nun konkret unter einer epiphänomenalen Eigenschaft am besten verstanden werden soll, wird zwar immer noch diskutiert (z.B. Baysan, 2018), trotz alledem zieht Gadenne (2006, S. 114) den Schluss, dass der Epiphänomenalismus immer noch die beste Option darstellt: "It follows from this considerations that QEP [qualia-epiphenomenalism] is at least as plausible as ist rivals. I even think QEP is more convincing, since it saves the most obvious assumption, namely, that Q-events [qualia] exist and have non-physical, intrinsic properties". Damit ist Gadenne in guter Gesellschaft, denn auch andere Autoren geben im Vergleich zwischen dem interaktionistischen Dualismus und dem Epiphänomenalismus Letzteren den Vorzug. So schreibt Jackson (1982, S. 135):

All right, there is no knockdown refutation of the existence of epiphenomenal qualia. But the fact remains that they are an excrescence. They do nothing, they explain nothing, they serve merely to soothe the intuitions of dualists, and 
it is left a total mystery how they fit into the world of science. In short we do not and cannot understand the how and why of them.

In ähnlicher Weise schreibt Chalmers (1996, S. 142), dass letztendlich der Epiphänomenalismus die beste dualistische Spielart darstellt und sich keine überzeugenden Gründe dagegen finden lassen:

\begin{abstract}
Any view that takes consciousness seriously will at least have to face up to a limited form of epiphenomenalism... The most common objection to epiphenomenalism is simply that it is counterintuitive or even "repugnant". Finding a conclusion counterintuitive or repugnant is not sufficient reason to reject the conclusion, however, especially if it is the conclusion of a strong argument. Epiphenomenalism may be counterintuitive, but it is not obviously false, so if a sound argument forces it on us, we should accept it.
\end{abstract}

\title{
6.2. Warum man ein Dualist sein soll
}

Falls nun jemand ein Dualist sein will, dann ist er gut beraten, ein epiphänomenaler Eigenschaftsdualist zu sein. Doch welche Gründe gibt es, einen epiphänomenalen Eigenschaftsdualismus zu vertreten? Das bekannteste und wohl meistdiskutierteste dualistische Argument ist das Argument des unvollständigen Wissens von Jackson (1982, 1986), denn es bringt die dualistischen Überzeugungen in einem lebhaften und intuitiv starken Gedankenexperiment zum Ausdruck. Genau deshalb stellt es, so der wahrgenommene Grundtenor innerhalb der Philosophie des Geistes, auch das überzeugendste Argument für den Dualismus dar. Denn wenn Jacksons Argument überzeugender ist als jedes andere dualistische Argument und der Physikalismus Jacksons Argument besiegt, dann besiegt der Physikalismus auch jedes andere dualistische Argument. Infolgedessen sehe ich Jacksons Argument auch als die letzte Verteidigungslinie für das dualistische Lager an. Auch andere dualistischen Argumente, die in vielerlei Hinsicht ähnlich zu Jacksons Argument sind, aber dennoch in gewissen Aspekten verschieden sind, werden nachfolgend angeführt. Viele Explikationen der Argumente stützen sich auf Gole (2008b).

Alle folgenden dualistischen Argumente haben gemeinsam, dass sie sich gegen jegliche Form des Eliminativismus richten, der immerhin einer von zwei Teilen meiner Explikation des Physikalismus darstellt. Genauso richten sie sich entweder ausschließlich gegen den A-prioriPhysikalismus oder gegen alle Spielarten des Physikalismus. Ich führe keine dualistischen Argumente auf, die sich ausschließlich gegen den A-posteriori-Physikalismus richten. Der Grund ist schlichtweg, dass ein Teil meiner Explikation des Physikalismus einen A-priori-Physikalismus 
umfasst und jedes dualistische Argument gegen den A-posteriori-Physikalismus nicht gegen meinen Standpunkt vorgebracht werden kann ${ }^{49}$.

\subsubsection{Die fast allwissende Mary}

Jackson $(1982,1986)$ stellt in seinem Gedankenexperiment Mary vor. Mary lebt in einem schwarz-weißen Raum und sie hat noch nie in ihrem Leben Farben gesehen. Nichtsdestotrotz ist Mary eine brillante Wissenschaftlerin mit Schwerpunkt in der Farbwahrnehmung und weiß alle physikalischen Fakten über Farbwahrnehmung, die es gibt. Sie weiß auch ganz genau, was im Gehirn dabei physiologisch vorgeht, und beschreibt in physikalischen Begriffen, was passiert, wenn man grünes Gras oder eine rote Rose betrachtet. Beispielsweise zeigt sie genau die Unterschiede auf zwischen den Wellenlängen der Farben Rot und Grün, sie beschreibt den neuronalen Weg von der Retina bis zum Okzipitallappen sowie all die anderen beteiligten physikalischen Prozesse. Plötzlich ist Mary die Möglichkeit geboten, ihr schwarz-weißes Zimmer zu verlassen. Sie verlässt ihren Raum und sieht zum ersten Mal in ihrem Leben grünes Gras und eine rote Rose. Nun die Frage, bei der sich die Meinungen und Intuitionen scheiden: Wird Mary neue Fakten lernen? Jackson bejaht diese Frage, denn es gibt nicht-physikalische Fakten in der Farbwahrnehmung ${ }^{50}$. Angelehnt an Nida-Rümelin (2019) ist die Struktur von Jacksons Argument wie folgt:

(1) Wenn der Physikalismus wahr ist, dann gibt es keine nicht-physikalischen Fakten hinsichtlich einer Farbwahrnehmung.

(2) Bevor Mary ihr schwarz-weißes Zimmer verlässt, weiß sie alle physikalischen Fakten, die es hinsichtlich einer Farbwahrnehmung gibt.

(3) Bevor Mary ihr schwarz-weißes Zimmer verlässt, gibt es bestimmte Fakten hinsichtlich einer Farbwahrnehmung, die sie nicht weiß.

(4) Ergo, es gibt nicht-physikalische Fakten hinsichtlich einer Farbwahrnehmung und der Physikalismus ist falsch.

Die Konklusion (4) soll zeigen, dass der Physikalismus nicht komplett ist, denn es wird etwas Wesentliches ausgelassen, nämlich das Wie-es-Ist eines bestimmten Erlebnisses. Der Physikalismus beschreibt demnach nicht alles, weil es Qualia als etwas Nicht-Physikalisches und Subjektives unserer Erlebnisse nicht beschreibt. Erst nachdem Mary ihr schwarz-weißes Zimmer verlässt, lernt sie dieses nicht-physikalische Faktum der Farbwahrnehmung und komplementiert damit ihr Wissen zu all den physikalischen Fakten der Farbwahrnehmung.

\footnotetext{
49 Die zwei bekanntesten Argumente ausschließlich gegen den A-posteriori-Physikalismus sind Whites Eigenschaftsdualismusargument (White, 1986, 2007) sowie Horgans und Tiensons Argument der Zerlegbarkeit (Horgan \& Tienson, 2001; Horgan, 2006).

50 Jackson lehnt erst in seinen späteren Arbeiten den Dualismus und sein Argument des unvollständigen Wissens ab und konviert zum Physikalisten (Jackson, 1998b).
} 
Im Allgemeinen ist die Frage diejenige, wie die Situation zu interpretieren ist, in der sich Mary nach Verlassen ihres schwarz-weißen Zimmers befindet. Jacksons Argument stellt gewissermaßen ein Dilemma dar. Räumt man ein, dass Mary ein neues Faktum lernt, dann ist das gesamte physikalische Wissen nicht genug, um alles zu wissen, das es zu wissen gibt. Infolgedessen ist der Wissenszuwachs aufgrund nicht-physikalischer bzw. mentaler Fakten zu erklären. Eine dualistische Philosophie des Geistes ist demnach wahr und der Physikalismus ist falsch. Sagt man aber, dass Mary keine neue Tatsache lernt, dann muss eine Erklärung innerhalb des physikalistischen Rahmens gegeben werden. Demnach bleibt die Intuition übrig, so Anhänger von Jacksons Argument, dass etwas Wesentliches ausgelassen wird, das die Naturwissenschaften nicht erklären können. Kritiken gegen Jacksons Argument, mit teilweise auch sehr technischen Einwänden, gibt es viele. Für eine Übersicht verweise ich an die Sammelbänder von Alter und Walter (2007) sowie Coleman (2019).

\subsubsection{Lückenhafte Erklärungen}

Levines (1983, 1993, 2018) Argument der Erklärungslücke fokussiert auf den vermeintlichen Graben, der zwischen physikalischen und mentalen Vorgängen existiert. Dieser Graben besteht in der empfundenen Asymmetrie zwischen der Erklärung physikalischer Vorgänge durch all unser physikalischen Wissen und der Erklärung mentaler Vorgänge durch all unser physikalisches Wissen. Ersteres stellt weitestgehend kein tieferes Problem dar, jedoch besteht im Letzteren eine Lücke. Unabhängig davon, wie entwickelt und elaboriert unsere physikalischen Theorien auch sind, sie bleiben uns dennoch eine Erklärung des Mentalen schuldig. Das Argument der Erklärungslücke lässt sich wie folgt explizieren:

(5) Wenn der Physikalismus wahr ist, dann erklärt er alle Vorgänge, die es gibt.

(6) Der Physikalismus erklärt nicht unsere mentalen Vorgänge.

(7) Es gibt mentale Vorgänge.

(8) Der Physikalismus ist falsch.

Betrachten wir Levines Argumentation anhand des klassischen Beispiels "Schmerz = C-FaserStimulation". Man kann alle Theorien und Erklärungen über eine C-Faser-Stimulation sammeln und versuchen, zu erklären, warum eine C-Faser-Stimulation genau die Eigenschaft hat, schmerzlich zu sein, oder wie ein Schmerz einen Ort einnehmen kann in derselben Art und Weise, wie eine C-Faser-Stimulation einen Ort einnimmt. Das Ergebnis ist, dass man es nicht genau erklären kann, warum physikalische Vorgänge schmerzlich sind oder wie ein Schmerz konkret verortet werden kann. Obwohl eine C-Faser-Stimulation einen Ort impliziert, ist es nicht der Fall bei Schmerzen, die aus intuitiven Gründen keinen Ort implizieren. Somit besteht hier eine Erklärungslücke. Betrachten wir zum Vergleich eine postulierte Identität zwischen zwei 
physikalischen Entitäten: "Wasser $=\mathrm{H}_{2} \mathrm{O}$ ". Hier findet sich keine Erklärungslücke, denn zu fragen, warum Wasser genau dieselben Eigenschaften aufweist wie $\mathrm{H}_{2} \mathrm{O}$, ist hier sinnlos. Denn überall, wo Wasser ist, ist auch $\mathrm{H}_{2} \mathrm{O}$. Beide Ausdrücke implizieren bereits intuitiv einen Ort. Der springende Punkt ist im Prinzip die Rolle der Intuition, welche die Identitätsaussage "Schmerz = C-Faser-Stimulation" infrage stellt, jedoch nicht die Identitätsaussage "Wasser $=\mathrm{H}_{2} \mathrm{O}$ ".

Es ist wichtig, zu betonen, dass Levine nicht die Absicht hatte, ein anti-physikalistisches Argument vorzulegen, denn eine Erklärungslücke alleine rechtfertigt nach ihm nicht, eine ontologische Konklusion wie in (8) zu ziehen. Levine hatte vielmehr im Auge, ein epistemisches Problem anzusprechen, für das es im physikalistischen Lager zurzeit keine adäquate Lösung gibt. Nichtsdestotrotz wurde im Laufe der Zeit auf dualistischer Seite argumentiert, dass sehr wohl aus einer Erklärungslücke auch ontologische Konklusionen gezogen werden können.

\subsubsection{Die Außenperspektive auf Fledermäuse}

Nagel (1974) ordnet seine Argumentation rund um die Unterscheidung zwischen Subjektivität und Objektivität an. Sein Fledermausargument beginnt damit, dass unsere Vorstellungskraft an unsere bisherigen Erlebnisse geknüpft ist. Jedes neue Erlebnis, das verschieden ist zu unseren bisherigen Erlebnissen, wie beispielsweise eine Fledermaus zu sein und die Beute durch ein Echolot zu orten, können wir uns nicht vollumfänglich vorstellen. Wir können beispielsweise aus der Dritte-Person-Perspektive beschreiben, wie ein Echolot einer Fledermaus funktioniert, welche Beute typischerweise geortet wird und dergleichen. Wir können uns aber nicht vorstellen, wie es ist, eine Fledermaus zu sein und die Beute durch ein Echolot zu orten. Ein FledermausErlebnis zu haben, ist völlig unterschiedlich zu den Erlebnissen, die wir als Mensch im Laufe unseres Lebens machen. Wir wissen, wie es ist, ein Mensch zu sein, aber wir wissen nicht aus der Erste-Person-Perspektive, wie es ist, eben genau diese Kreatur zu sein. Ein Wissen aus der Erste-Person-Perspektive wird nicht durch ein Wissen aus der Dritte-Person-Perspektive erfasst. Dadurch können wir auch nicht wissen, wie der Physikalismus, als etwas aus der objektiven, Dritte-Person-Perspektive, eine Erklärung für Qualia geben kann, die etwas aus der subjektiven, Erste-Person-Perspektive sind. Ein dualistisches Argument lässt sich nun wie folgt darlegen:

(9) Wenn der Physikalismus wahr ist, dann kann alles aus der Dritte-Person-Perspektive gewusst werden.

(10) Zu wissen, wie es ist, eine Fledermaus zu sein, kann nur aus der Erste-PersonPerspektive gewusst werden.

(11) Der Physikalismus ist falsch.

Nagels Argument zeigt, dass nur dann gewusst werden kann, wie es ist, eine Fledermaus zu sein, wenn man auch selbst eine Fledermaus ist. Es ist genau diese Subjektivität, die sich der 
physikalischen Geschichte der Welt entzieht. Der Physikalismus fordert jedoch, dass alles objektiv ist, denn alles Existierende ist physikalisch.

\subsubsection{Was Zombies nicht haben}

Chalmers (1996) hat das Zombie-Argument salonfähig gemacht, in dem der Dreh- und Angelpunkt unser Verständnis der modalen Begriffe "Vorstellbarkeit" und "Möglichkeit" ist. Ich möchte an dieser Stelle einen philosophischen Zombie einführen, der ein physikalisches Duplikat von mir ist, aber mit dem einen Unterschied. Mein Zombie hat keine Gefühle, keine Wünsche, kein mentales Leben wie auch immer ein mentales Leben aussehen könnte. Chalmers' dualistisches Argument geht davon aus, dass wir uns widerspruchsfrei einen Zombie vorstellen können. Weil wir uns einen Zombie vorstellen können, ist ein Zombie auch möglich. Die Möglichkeit von Zombies ist mit dem Physikalismus unvereinbar, weshalb dadurch die Falschheit des Physikalismus folgt. Hier ist mit "möglich" eine metaphysische Möglichkeit gemeint, sodass es Zombies in unserer aktualen Welt geben könnte. Angelehnt an Chalmers (2010) lässt sich das Argument folgendermaßen rekonstruieren:

(12) Wenn der Physikalismus wahr ist, dann ist es unmöglich, dass es Zombies gibt.

(13) Es ist vorstellbar, dass es Zombies gibt.

(14) Wenn Zombies vorstellbar sind, dann sind Zombies auch möglich.

(15) Zombies sind möglich und der Physikalismus ist falsch.

Vorläufer dieses Arguments sind bereits bei Descartes (1641) zu finden, der durch eine ähnliche Argumentation auf zwei Substanzen schließt. Für Descartes ist es vorstellbar, dass Körper und Geist als Substanzen getrennt voneinander existieren können, denn ich kann mich als mentales Subjekt ohne physische Eigenschaften denken. Wenn man sich solch ein Szenario vorstellen kann, dann ist es auch möglich. So wie weiter oben beschrieben, ist man nun im letzten Schritt dazu berechtigt, auf die Existenz von zwei Substanzen zu schließen. Ebenso überlegte in gleicher Manier Locke (1689), ob jemand anderes auch bei der Betrachtung von Gegenständen denselben Farbeindruck hat oder das Farbspektrum invertiert sein könnte. Ein invertiertes Farbspektrum würde bedeuten, dass ich die Farbe Blau bei Betrachtung des Himmels sehe, mein physikalisches Duplikat jedoch die Farbe Rot. Wenn dieses Szenario vorstellbar wäre, dann wäre es auch möglich und sohin wäre auch in dieser Argumentationslinie der Physikalismus falsch. In der gegenwärtigen Literatur hat insbesondere Kirk (1974a,b) die erste Version eines Zombie-Arguments vorgelegt und Kripke (1980) hat bereits gesagt, dass man sich vorstellen kann, dass Schmerzen nicht mit einer C-Faser-Stimulation einhergehen. Da das Zombie-Argument als ein Modalargument auf ein spezifisches Verständnis der Begriffe "Vorstellbarkeit" und "Möglichkeit" beruht, wurde es auch deshalb von einigen Dualisten als 
weniger attraktiv angesehen, sogar von Jackson selbst (1982, S. 131): "The trouble rather with the modal argument is that it rests on a disputable modal intuition. Disputable because it is disputed".

\subsubsection{Qualia begreifen können}

Nida-Rümelin (2007) entwickelte auf Basis des Verhältnisses zwischen mentalen Begriffen und den zugehörigen mentalen Eigenschaften einerseits sowie auf Basis des Verhältnisses zwischen mentalen und physikalischen Begriffen andererseits ein neues dualistisches Argument. Sie verwendet den Ausdruck "phänomenale Eigenschaft", um sich auf ein Quale als eine mentale Eigenschaft zu beziehen, und "phänomenaler Begriff", um sich auf einen mentalen Begriff zu beziehen, der ein Quale als Referenten hat. Ich werde mich zur Explikation ihres Arguments an ihre bevorzugten Ausdrücke halten.

Im Kern ihres Arguments liegt die Unterscheidung zwischen "eine Eigenschaft haben" (having a property) und "eine Eigenschaft begreifen" (grasping a property). Eine Eigenschaft zu begreifen bedeutet, zu verstehen, worin das Essentielle einer Eigenschaft besteht (S. 307): "To grasp a property is to understand what having that property essentially consists in". Das Essentielle einer Eigenschaft wird wiederum dann verstanden, wenn der Referent eines phänomenalen Begriffes in einer beliebigen möglichen Welt bekannt ist. Basierend auf NidaRümelin (2007) lässt sich das Argument wie folgt darstellen:

(16) Wenn der Physikalismus wahr ist, dann sind phänomenale Eigenschaften identisch mit physikalischen Eigenschaften.

(17) Phänomenaler Essentialismus: Phänomenale Eigenschaften werden durch phänomenale Begriffe begriffen.

(18) Prinzip der kognitiven Transparenz: Wenn eine Eigenschaft durch zwei verschiedene Begriffe begriffen wird, dann kann rational geurteilt werden, ob diese zwei Begriffe notwendigerweise koextensiv sind.

(19) Kognitive Unabhängigkeit phänomenaler und physikalischer Begriffe: Ein physikalisches Wissen alleine reicht nicht aus, um rational zu urteilen, ob phänomenale und physikalische Begriffe notwendigerweise koextensiv sind.

(20) Kognitive Zugänglichkeit zu physikalischen Eigenschaften: Physikalische Eigenschaften werden durch physikalische Begriffe begriffen.

(21) Phänomenale Eigenschaften sind nicht identisch mit physikalischen Eigenschaften und der Physikalismus ist falsch.

Nida-Rümelins Argument hat seinen Dreh- und Angelpunkt darin, dass die Identität zwischen phänomenalen und physikalischen Eigenschaften durch die unterschiedliche Art und Weise 
blockiert wird, wie phänomenale und physikalische Begriffe referieren. So wie ich ihr Argument verstehe, wird die Referenz physikalischer Begriffe durch das physikalische Wissen festgelegt. Hingegen wird die Referenz phänomenaler Begriffe nicht ausschließlich durch physikalisches Wissen festgelegt, weil noch das Erleben hinzukommen muss. Damit sind phänomenale und physikalische Begriffe nicht koextensiv und deshalb auch nicht identisch oder synonym. Auf Basis dessen zieht Nida-Rümelin die Konklusion, dass ebenfalls phänomenale und physikalische Eigenschaften nicht identisch sind.

\subsection{Warum man ein Physikalist sein soll}

Die These des Physikalismus ist auf Basis der vorhergehenden Kapitel so zu verstehen, dass, falls der Physikalismus wahr ist, dann die erarbeitete Explikation des Physikalismus in (P1C) wahr ist. So wurde insbesondere in den Kapiteln 3 bis 5 argumentiert, dass die erarbeitete Explikation des Physikalismus die beste Version einer physikalistischen Position ist im Vergleich zu allen anderen physikalistischen Positionen. Die konkurrierenden physikalistischen Positionen waren der Behaviorismus, der Repräsentationalismus/Intentionalismus, der Fundierungsphysikalismus und bestimmte Spielarten des Funktionalismus. Das ist als erster Schritt wichtig, um zu einer bewertbaren These des Physikalismus zu kommen. Doch es kann nicht der letzte Schritt sein, weil es noch die Frage zu beantworten gilt, ob die erarbeitete Explikation des Physikalismus überhaupt wahr ist und sich damit auch gegen dualistische Positionen behaupten kann. Überraschenderweise ist die Auswahl an Argumenten für den Physikalismus sehr bescheiden, lediglich eine Handvoll klassischer Argumente, großteils noch aus der Gründungszeit des Physikalismus in der zweiten Hälfte des 20. Jahrhunderts, werden typischerweise vorgebracht.

\subsubsection{Die Identität als beste und einfachste Erklärung}

Vor allem Smart (1959), Feigl (1958/1967) und später auch Hill (1991), Block und Stalnaker (1999) sowie McLaughlin (2001) argumentieren aufgrund der Korrelation zwischen mentalen und physikalischen Vorgängen, dass die beste und einfachste Erklärung deren Identität ist. Der Ausgangspunkt von Smart war lediglich die Postulierung, dass es die einfachste Erklärung sei, ohne jedoch diesen Punkt viel weiter zu entwickeln. Hingegen brachten Nachfolger von Smart den weiteren Aspekt hinzu, dass die Identität nicht nur die einfachste Erklärung ist, sondern ebenso die beste Erklärung für den Zusammenhang des Mentalen und des Physikalischen liefert. Was als beste Erklärung gilt, nämlich "M = P", ist im Grunde das Ergebnis eines Vergleiches mit allen anderen Erklärungen. Anschließend wird abduktiv auf die Wahrheit von "M = P" geschlossen. Denn wenn die Annahme " $M$ = P" die Korrelation besser als andere Lösungsvorschläge erklärt, dann sollten wir auch die Wahrheit dieses Satzes annehmen. 
Diese Argumentationslinie blieb nicht unkommentiert und wurde stark von Kim (2005) kritisiert. Kim meint, dass eine Identität zwischen zwei Dingen eigentlich nichts erklärt, sondern lediglich zu einem Umschreiben einer Prämisse führt. Betrachten wir folgenden Schluss analog zu Kims Prämissen (S. 132):

(A) Stephen King ist ein erfolgreicher Autor.

(B) Stephen King = Richard Bachman.

(C) Ergo, Richard Bachman ist ein erfolgreicher Autor.

Die Konklusion $(C)$ schreibt lediglich Prämisse $(A)$ um und fügt nichts Neues hinzu. Doch Kim argumentiert, dass bei einer Erklärung etwas Neues hinzugefügt werden muss, um als Erklärung zu gelten. Somit schlussfolgert Kim, dass die Annahme einer Identität nicht durch den Rekurs auf dessen Erklärungsgehalt erfolgreich sein kann.

In Kims Argument steckt eine große intuitive Kraft. Glücklicherweise geht der Kelch, eine Replik gegen Kims Argument geben zu müssen, bei einem A-priori-Physikalisten vorüber. Der Grund ist, dass dieses Argument klassisch mit dem A-posteriori-Physikalismus und der Rechtfertigung von Identitätsaussage a posteriori verbunden ist. Wie ich in Kapitel 4 in der Diskussion über Argumente für den A-priori-Physikalismus gezeigt habe, folgt für Vertreter dieser Spielart des Physikalismus die Identität zwischen $\mathrm{M}$ und $\mathrm{P}$ deduktiv aus $\mathrm{P}$. Es muss somit nicht auf den erklärenden Charakter der Identitätsaussage im Sinne einer Abduktion zurückgegriffen werden, um sie zu rechtfertigen. Es muss stattdessen lediglich auf die deduktive Ableitung der Identitätsaussage aus dem gesamten physikalischen Wissen zurückgegriffen werden. Zwar habe ich ebenso in Kapitel 4 argumentiert, dass die Identitätsthese den Zusammenhang zwischen $\mathrm{M}$ und $\mathrm{P}$ am besten erklärt, doch wurde der Erklärungsgehalt der Aussage " $\mathrm{M}=\mathrm{P}$ " in der weiteren Diskussion als etwas verstanden, dass im Vergleich zu den anderen physikalistischen Positionen die beste Erklärung im Sinne der Einfachheit und der Sparsamkeit liefert. Es wird unter diesem Blickwinkel klar, dass der Begriff der besten Erklärung mehrdeutig ist: Kim versteht darunter etwas anderes als ich. Deshalb greift Kims Argument nicht für die Version eines A-priori-Physikalismus, wie ich inn eingeführt habe.

Hinsichtlich Smarts Sichtweise, dass eine Identitätstheorie einfacher ist, lässt sich jedoch sehr wohl ein Konnex zum hier vertretenen A-priori-Physikalismus herstellen. Es ist unumgehbar, dass näher beschrieben werden muss, was letztendlich "einfacher" bedeutet. Ich habe das Merkmal der Einfachheit als ein Kriterium wissenschaftlicher Theorien eingeführt, wobei "einfacher zu sein" insbesondere bedeutet, "kognitiv zugänglicher zu sein", "rascher verstehbar zu sein", "übersichtlicher dargestellt zu sein" oder auch "eindeutiger erfassbar zu sein". Dies macht es möglich, nun auch die Identitätstheorie mit dualistischen Positionen zu vergleichen, so wie ursprünglich die Identitätstheorie mit den konkurrierenden physikalistischen Positionen verglichen wurde. 


\subsubsection{Die Identität als Konklusion eines deduktiven Arguments}

In diese Kategorie fallen vor allem die Arbeiten von Lewis (1966) und Armstrong (1968). Beide argumentieren, dass Schmerzen eine gewisse kausale Rolle einnehmen und diese kausale Rolle wiederum durch Gehirnvorgänge eingenommen wird. Dadurch ist Schmerz nichts anderes als ein Gehirnvorgang. Hier folgt die Identität " $M=P$ " deduktiv aus ihren Prämissen:

(D) Schmerz = derjenige Zustand, der die Schmerz-Rolle einnimmt.

(E) C-Faser-Erregung = derjenige Zustand, der die Schmerz-Rolle einnimmt.

(F) Ergo, Schmerz = C-Faser-Erregung.

Dieses Argument ist uns bereits in Kapitel 3 in der Diskussion des Funktionalismus begegnet, deshalb werde ich nachfolgend nicht den Funktionalismus nochmals diskutieren, sondern die Aufmerksamkeit auf die Art und Weise lenken, wie formal zur Konklusion (F) gelangt wird. In Prämisse (D) wird a priori festgestellt, dass Schmerzen zu haben bedeutet, die Rolle von Schmerzen einzunehmen. Die Schmerz-Rolle besteht aus der verbale Äußerung von "Es schmerzt!", dem Wegziehen der Hand von der heißen Herdplatte und dergleichen. Dieser Schritt ist uns aus dem analytischen Funktionalismus bereits sehr gut bekannt und ist dadurch gerechtfertigt, weil wir bereits den Begriff "Schmerz" hinreichend verstehen. In Prämisse (E) zeigen uns die Erkenntnisse der physikalischen Wissenschaften, dass eine C-Faser-Erregung genau das auslöst, was Schmerzen ausmacht, nämlich die verbale Äußerung von "Es schmerzt!", dem Wegziehen der Hand von der heißen Herdplatte und dergleichen. Als Konklusion folgt $(F)$ deduktiv aus den beiden Prämissen.

Trotz der Stärke dieses Arguments und der Tatsache, dass es sich um ein gültiges, deduktives Argument handelt, wurde dieser Ansatz von Lewis und Armstrong insbesondere aufgrund der ersten Prämisse (D) angegriffen. Die Einwände dagegen stellen die Synonymie zwischen dem M-Begriff und dem P-Begriff infrage, woraus sich alternative physikalistische Positionen entwickelt haben, unter denen die häufigsten in Kapitel 3 dargestellt sind. Aber auch außerhalb des physikalistischen Lagers kam weitere Kritik (z.B. Chalmers, 1996).

\subsubsection{Die Identität als Konklusion des Arguments der kausalen Geschlossenheit}

Ein weiterer Spezialfall eines Arguments, das die Identität von $\mathrm{M}$ und $\mathrm{P}$ deduktiv als Konklusion hat, ist Papineaus (2002) Argument der kausalen Geschlossenheit des Physikalischen, das nur den Schluss auf physikalische Ursachen zulässt.

(G) Mentale Vorgänge haben physikalische Wirkungen. 
(H) Alle physikalischen Wirkungen haben notwendige und hinreichende physikalische Ursachen.

(I) Alle physikalischen Wirkungen sind nicht systematisch überdeterminiert.

(J) Ergo, mentale Vorgänge sind physikalische Vorgänge.

In der ersten Prämisse $(G)$ wird aufgrund unserer alltäglichen Erfahrung festgestellt, dass unser Dursterlebnis uns dazu bringt, etwas zu trinken, oder dass unser Gefühl von Liebe uns dazu bringt, die geliebte Person zum Essen auszuführen. Die zweite Prämisse $(H)$ enthält die Kernaussage des Prinzips der kausalen Geschlossenheit, nämlich die, dass jede physikalische Wirkung eine notwendige hinreichende physikalische Ursache hat. Die dritte Prämisse (I) schließt eine systematische Überdeterminiertheit aus. Ist ein Ereignis überdeterminiert, dann verursachen zwei hinreichende Gründe dasselbe Ereignis. Ein Beispiel verwendend bedeutet das, dass der Tod eines Tieres von zwei hinreichenden Gründen gleichzeitig herbeigeführt wird, wenn ein Jäger mit seiner Schrotflinte darauf schießt und ein Teil der Schrotkugeln den Kopf und der andere Teil der Schrotkugeln das Herz des Tieres trifft. Beide Verletzungen sind für sich genommen hinreichend für das Erlegen des Tieres. Das Problem dabei ist, diese Ausnahme als die Regel anzunehmen. Solch ein Szenario kann prinzipiell vorkommen, aber anzunehmen, dass in gleicher Weise die Kausalität funktioniert, ist keine vernünftige Option. Es ist deswegen unvernünftig, weil damit eingeräumt werden würde, dass eine physikalische Wirkung immer mehr als eine hinreichende Ursache hat, und zwar eine physikalische und eine mentale Ursache. Schließlich folgt aus diesen Prämissen die Konklusion $(\mathrm{J})$ und damit die Identität "M = P".

Kim (2005) selbst und andere Autoren (z.B. Tiehen, 2018) sehen im Argument der kausalen Geschlossenheit das vielversprechendste und einflussreichste Argument für den Physikalismus, andere wiederum meinen, dass es bei näherer Betrachtung etwas an Glanz verliert (Stoljar, 2010), und wiederum andere meinen, dass es ein zirkuläres Argument sei (Bishop, 2006). Ich möchte nachfolgend insbesondere Bishops Einwand näher beleuchten, der eine versteckte Prämisse (VP) im Argument der kausalen Geschlossenheit sieht:

(VP) Nur physikalische Ursachen können Ursachen sein.

Nur dann, wenn auch (VP) wahr ist, kann Papineaus Argument angenommen werden. Bishop argumentiert jedoch, falls nun (VP) angenommen wird, dann wird Papineaus Argument zirkulär, weil die Wahrheit des Physikalismus und die Wahrheit von (VP) nicht unabhängig voneinander akzeptiert werden können. Somit setzt (VP) bereits die Wahrheit des Physikalismus voraus, doch (VP) ist als weitere Prämisse notwendig, um die Wahrheit des Physikalismus überhaupt zu begründen. 
Alles in allem denke ich jedoch nicht, dass Bishop zur Gänze Recht hat. Denn die Wahrheit von (VP) kann ganz leicht unabhängig von der Wahrheit des Physikalismus akzeptiert werden. So akzeptieren beispielsweise Epiphänomenalisten problemlos (VP) und erweitern unsere Ontologie um kausal impotente, nicht-reduzierbare mentale Eigenschaften. Papineau selbst hat dieses Problem mit seiner ersten Prämisse $(G)$ bereits gesehen. Hier kommt nun der Teil, mit dem Bishop Recht behalten hat. Papineaus Argument ist trotzdem zirkulär, weil ein Epiphänomenalismus und jeglicher andere nicht-interaktive Dualismus ausgeschlossen werden muss, um die erste Prämisse $(G)$ annehmen zu können, doch das ist wiederum nur dann möglich, wenn der Physikalismus wahr ist. Genau dieser Punkt soll ja vom Argument der kausalen Geschlossenheit gezeigt werden. Somit setzt die Wahrheit von Prämisse (G) bereits die Konklusion (J) voraus (siehe zu diesem Punkt auch Lycan, 2009).

\subsubsection{Die Identität als die wahrscheinlichste Erklärung}

Ein neues Argument für die Identitätstheorie legt Aranyosi (2011) vor, der probabilistisch argumentiert, sodass die Identitätstheorie die wahrscheinlichste Erklärung ist. Aranyosi geht von folgender Disjunktion aus, die den Zusammenhang zwischen $\mathrm{M}$ und $\mathrm{P}$ darlegt: Entweder treten $\mathrm{M}$ und $\mathrm{P}$ als ontologisch verschiedene Entitäten immer gesetzesartig gemeinsam auf oder $\mathrm{M}$ und $P$ sind ein und dasselbe und treten deshalb immer gesetzesartig gemeinsam auf. Die erste Option postuliert psychophysische Gesetzmäßigkeiten, die notwendigerweise zwischen verschiedenen Entitäten gelten und sich aus unserer Introspektion ergeben. Die zweite Option postuliert eine Identitätsrelation zwischen $M$ und $P$. Aranyosi argumentiert sodann für die zweite Option, indem er die erste Option ausschließt:

(K) Entweder ist eine notwendige psychophysische Relation zwischen den verschiedenartigen Entitäten $\mathrm{M}$ und $\mathrm{P}$ gegeben oder es ist die Identitätstheorie wahr.

(L) Es ist keine notwendige psychophysische Relation zwischen den verschiedenartigen Entitäten $\mathrm{M}$ und $\mathrm{P}$ gegeben, falls Humes Diktum gilt oder Ockhams Rasiermesser gilt oder keine unerklärbaren und primitiven Notwendigkeiten postuliert werden.

(M) Es gilt zumindest eine der folgenden Überlegungen, sodass (Ma) Humes Diktum gilt oder (Mb) Ockhams Rasiermesser gilt oder (Mc) keine unerklärbaren und primitiven Notwendigkeiten postuliert werden.

(N) Ergo, die Identitätstheorie ist wahr.

Hier ist die dritte Prämisse (M) interessant. Aranyosi schließt in seiner Argumentation keines der Überlegungen (Ma) bis (Mc) ein für alle Mal aus, sondern er geht vorsichtiger vor und sagt, dass die Wahrscheinlichkeit der Gültigkeit von (Ma) bis (Mc) höher ist als deren Nicht-Gültigkeit. 
Humes Diktum in (Ma) besagt, dass es keine notwendige Verbindung zwischen komplett verschiedenen Entitäten gibt. Dieses Diktum ist beliebt innerhalb der Metaphysik, obwohl klare Argumente dafür typischerweise fehlen (Wilson, 2010), dennoch ordnet Aranyosi dessen Wahrheit eine höhere Wahrscheinlichkeit zu als dessen Falschheit. Ockhams Rasiermesser in $(\mathrm{Mb})$ ist uns bereits in Kapitel 4 begegnet und deckt die ontologische Sparsamkeit ab. Für Aranyosi ist es wahrscheinlicher, dass eine ontologisch einfachere Theorie angenommen wird als eine ontologisch komplexere Theorie.

Die Forderung in (Mc), keine unerklärbaren und primitiven Notwendigkeiten zu postulieren, ist uns ebenfalls in einem etwas anderen Gewand in Kapitel 4 begegnet. Es wurde in Kapitel 4 im Zuge der Diskussion zwischen dem A-priori-Physikalismus und dem A-posterioriPhysikalismus angesprochen und zeigt, wie in beiden Lagern zur Identitätsaussage " $M=P$ " gelangt wird. Der A-posteriori-Physikalismus postuliert eine primitive Identität aufgrund einer Abduktion, wohingegen der A-priori-Physikalismus eine Identität aufgrund einer Deduktion postuliert. Ähnliches spielt sich auch in Aranyosis Kontext ab, der eine höhere Wahrscheinlichkeit bestimmten Notwendigkeiten zuordnet, die erklärbar und deduktiv ableitbar sind, als Notwendigkeiten, die nicht erklärbar und primitiv sind. Schlussendlich folgt deduktiv aus der Falschheit des ersten Gliedes der Disjunktion in Prämisse (K) die Wahrheit der Identitätstheorie in $(\mathrm{N})$.

\subsubsection{Der Physikalismus als Standardsicht}

Die letzte Argumentationslinie für die Wahrheit des Physikalismus ist im Grunde kein Argument. Es ist vielmehr eine Umkehr der Beweislast zulasten des Dualisten und der Ausdruck einer tiefen Überzeugung. Physikalisten in diesem Lager sagen, dass der Physikalismus als die Standardsicht angenommen werden soll und dadurch auch gleichzeitig die Wahrheit dessen akzeptiert werden soll. Es liegt primär an den Gegnern des Physikalismus, argumentativ zu zeigen, warum physikalistische Positionen falsch sein sollen. Ohne solche Argumente sei man getrost berechtigt, bei seiner Position zu verweilen.

Hier wird die Bürde des Beweises einfach an den Dualisten übergeben. Bei argumentativem Erfolg des Dualisten käme es dem Widerlegen einer eingefahrenen Sicht auf die Welt, einer Weltanschauung, nahe. Gillett und Loewer (2001, S. IX) eröffnen im Vorwort ihres Sammelbandes die Diskussion durch die Betonung des Aspektes der Weltanschauung, wenn vom Physikalismus die Rede ist: "Every era has its weltanschauung and in much contemporary philosophy the doctrine of 'physicalism' plays this role". Der Begriff der Weltanschauung ist stark und legitimiert beinahe schon alleine die Akzeptanz des Physikalismus ohne weitere positive Argumente. Ähnlich sichert Dennett (2016) den Status für seinen Eliminativismus, der als Standardtheorie gelten sollte und nur durch starke Gegenargumente für eine rivalisierende Theorie aufgegeben werden sollte. 
Zu dieser Verteidigungsstrategie lässt sich sagen, dass sie sehr wohl von beiden Lagern angewandt werden kann. So könnte beispielsweise der Dualist sagen, dass durch die alltägliche introspektive Erfahrung das Erleben von Gefühlen, Gedanken und allen weiteren mentalen Zuständen selbstevident ist und wir im Sinne von Descartes eine denkende Substanz sind oder im Sinne von Eigenschaftsdualisten fundamental mentale Eigenschaften aufweisen. Aus diesem Grunde, so könnte der Dualist weiter ausführen, ist eine dualistische Theorie die gültige Standardtheorie in der Philosophie des Geistes und der Physikalist hat die Bürde des Beweises, das Gegenteil zu zeigen. An diesem Punkt wird klar, dass ein Verharren auf dieser Position zu einer Patt-Stellung führt und keines der beiden Lager einen Fortschritt verzeichnen kann.

\subsection{Zusammenfassung}

Ich habe in diesem Kapitel die zwei Kontrahenten um eine adäquate Bewusstseinstheorie gegenübergestellt, indem ich die einflussreichsten und gängigsten Argumente für den Dualismus auf der einen Seite und für den Physikalismus auf der anderen Seite expliziert habe. Diese Gegenüberstellung soll dazu dienen, die Argumente für die jeweiligen Positionen besser zu verstehen. In der ersten Annäherung an den Dualismus habe ich begonnen, zwischen einem Substanzdualismus und einem Eigenschaftsdualismus zu unterscheiden. Die erste Spielart ist die logisch stärkere Form, doch die zweite Spielart hat sich bei den meisten zeitgenössischen Dualisten durchgesetzt. Sohin habe ich den interaktionistischen und epiphänomenalen Dualismus verglichen, wobei ein Interaktionismus den alltäglichen Intuitionen von kausal wirksamen mentalen Entitäten entspricht. Ein Epiphänomenalismus hingegen geht von kausal impotenten mentalen Entitäten aus und geht so dem Problem aus dem Wege, in ganz konkreter Art und Weise darzulegen, wie eine Interaktion überhaupt zustande kommen kann. Insgesamt scheint sich ein epiphänomenalistischer Dualismus am ehesten durchzusetzen. Diese Diskussion zusammennehmend stellt damit der epiphänomenalistische Eigenschaftsdualismus die überzeugendste dualistische Position dar. Im Anschluss an diese Erkenntnis habe ich die fünf bekanntesten Argumente für den Dualismus gebracht, unter denen Jacksons Argument des unvollständigen Wissens sich als das am meisten diskutierte herausstellt. Schließlich habe ich dieses Kapitel damit geschlossen, die fünf bekanntesten Argumente für den Physikalismus herauszuarbeiten. 


\section{Let's get physical, physical I wanna get physical}

Olivia Newton-John (1981) 


\section{Die Wahrheit des Physikalismus}

Ich werde in diesem Kapitel als vorrangiges Ziel ein neues Argument für den Physikalismus vorstellen, das die (vorläufige) Wahrheit des Physikalismus deduktiv aus drei Prämissen folgert. Jede der drei Prämissen wird aus dem gegenwärtigen Diskussionsstand hergeleitet und erfolgreich verteidigt. Dieses Kapitel schließt mit einem möglichen Einwand gegen das generalistische Argument für den Physikalismus und dem Vorwurf, dass sich das Argument lediglich gegen dualistische Positionen richtet, worunter der epiphänomenalistische Eigenschaftsdualismus die plausibelste Position darstellt. Mein Physikalismusargument ist jedoch nicht gegen alternative monistische Positionen wie den Idealismus, den neutralen Monismus und den Panprotopsychismus wirksam. Ich kontere zum einen durch das Vorbringen einer Familie von Kombinationsproblemen, die gegen diese alternativen monistischen Lager sprechen. Zum anderen entwickle ich ein neues Argument durch die Anwendung des Satzes von Bayes (Bayes, 1764), das die Wahrscheinlichkeit für die Wahrheit des Physikalismus höher einschätzt als die Wahrheit der rivalisierenden monistischen Positionen.

\subsection{Das generalistische Argument für den Physikalismus}

Ich möchte hier ein neues deduktives Argument für den Physikalismus vorstellen, das ich das generalistische Argument für den Physikalismus nenne. Das Argument ist aus zweierlei Gründen generalistisch. Erstens, es ist deshalb ein generalistisches Argument, weil es sowohl den A-priori-Physikalismus als auch den Eliminativismus stützt, denn eine Disjunktion aus beiden Position ist die Explikation des Physikalismus, wie ich inn vertrete. Immer dann, wenn ich von "Physikalismus" spreche und ich für dessen Wahrheit argumentiere, meine ich damit die spezielle Form des Physikalismus, wie er in den Kapiteln 2 bis 5 erarbeitet und in (P1c) zusammengefasst wurde. Zweitens, es fasst alle bisherigen empirischen und philosophischen Erkenntnisse in einem einzigen Argument zusammen. Dabei stütze ich mich unter anderem auf viele der bisherigen positiven Argumente für den Physikalismus und verbinde sie in einer neuen Art und Weise miteinander. Ich argumentiere darauffolgend auf Basis der Überzeugungskraft des Physikalismus für dessen Wahrheit. Das generalistische Argument für den Physikalismus ist folgendes:

(1) Wenn der Physikalismus die überzeugendste Position in der Philosophie des Geistes ist, dann soll der Physikalismus als wahr akzeptiert werden.

(2) Wenn der Physikalismus

(2a) die Kriterien wissenschaftlicher Theorien besser als der Dualismus erfüllt und 
(2b) die klassischen dualistischen Gedankenexperimente besser als der Dualismus erklärt und

(2c) durch wissenschaftliche Erkenntnisse besser als der Dualismus gestützt wird,

dann ist der Physikalismus die überzeugendste Position in der Philosophie des Geistes.

(3) Der Physikalismus

(3a) erfüllt die Kriterien wissenschaftlicher Theorien besser als der Dualismus und

(3b) erklärt klassische dualistische Gedankenexperimente besser als der Dualismus und

(3c) wird durch wissenschaftliche Erkenntnisse besser als der Dualismus gestützt.

(4) Der Physikalismus soll als wahr akzeptiert werden. | Folgt aus (1) bis (3).

Bei Gültigkeit der Prämissen des generalistischen Arguments für den Physikalismus folgt die Konklusion deduktiv. Nachfolgend möchte ich Gründe liefern, warum die Prämissen (1) bis (3) angenommen werden sollen. Als würdigen Hauptgegner gegen den Physikalismus werde ich die überzeugendste dualistische Position wählen, nämlich den epiphänomenalistischen

Eigenschaftsdualismus. Wenn ich also nachfolgend allgemein von einem Dualismus spreche, dann meine ich genau diesen, außer ich beziehe mich explizit auf andere Spielarten.

\subsubsection{Warum das Überzeugendste wahr sein soll - Prämisse (1)}

Hier geht es um die Verbindung zwischen der Aussage, die überzeugendste Position in der Philosophie des Geistes zu sein, und der Annahme, dass eben genau diese überzeugendste Position auch wahr sein soll. Dass der Physikalismus wahr ist, ist grundsätzlich eine metaphysische Aussage, weil der Physikalismus die intrinsische Natur alles Existierenden als physikalisch festmacht. Diese intrinsische physikalische Natur von Dingen lässt sich jedoch nicht direkt beobachten, es kann nur durch Beobachtung indirekt darauf geschlossen werden. Dafür muss eine Bedingung geliefert werden, unter deren Gültigkeit auf die Wahrheit des Physikalismus geschlossen werden kann. Dabei ist ebenso wichtig, dass diese Bedingung von Anhängern beider Lager, also von Physikalisten und Dualisten, akzeptiert werden kann. Es geht also darum, wann etwas als wahr angenommen werden soll. Wenn man vom Begriff der Wahrheit spricht, dann gibt es dafür bereits gute Definitionen, beispielsweise die Entsprechung von gedanklichen Vorstellungen mit der Wirklichkeit im Sinne der Korrespondenztheorie der Wahrheit oder darauf aufbauend die Entsprechung einer Aussage mit der Wirklichkeit im Sinne der semantischen Theorie der Wahrheit (Tarski, 1936).

Die Akzeptanz eines korrespondenztheoretischen Ansatzes birgt Konsequenzen für die Beurteilung der Prämisse (1), denn in diesem Fall ist die erste Prämisse keine logisch-analytische Wahrheit. Es kommt vielmehr darauf an, inwieweit eine überzeugendste 
Theorie auch tatsächlich den Beobachtungen entspricht, und damit wird es dem ersten Anscheine nach zu einer empirischen Wahrheit. Doch auch das ist nicht ganz korrekt. Sehen wir uns hierfür Beispiele aus der Geschichte der Naturwissenschaften an. So war beispielsweise vor Galileo die überzeugendste astronomische Theorie, dass die Erde im Mittelpunkt des Universums stehe, und vor Einsteins Relativitätstheorie war die überzeugendste Theorie, dass die Zeit eine lineare Konstante sei, völlig unabhängig von jedwedem Raum. Beide waren jedoch falsch und entsprachen nicht der Wirklichkeit, denn sie wurden von anderen, noch besseren Theorien abgelöst. Diese Beispiele zeigen, dass auch noch so überzeugende Theorien nicht notwendigerweise wahr sind. Gleichzeitig zeigen diese Beispiele auch, dass sie ganz im Popper'schen Sinne zu einer bestimmten Zeit rationalerweise und vorläufig als wahr angenommen worden sind. Hierfür möchte ich zwei Gründe anführen, warum die überzeugendste Position auch als wahr akzeptiert werden soll.

Erstens, wenn ich zwischen mehreren Positionen wählen muss, dann muss eine zumindest als vorläufig wahr angenommen werden und üblicherweise gewinnt die überzeugendste Position das Rennen um die (vorläufige) Wahrheit. So wie es auch bei einem Pferderennen einen Gewinner geben muss und üblicherweise das schnellste Pferd der Gewinner ist. Es ist weder das schönste noch das größte Pferd, auch ist es nicht das schnellste Pferd in jeder möglichen Welt zu jedem beliebigen Zeitpunkt, welches das Rennen macht, sondern schlichtweg das schnellste im Vergleich zu allen anderen Pferden, die am Rennen teilgenommen haben. Auf unsere gegenwärtige Situation übertragen, ist es gleichsam weder die bekannteste Position noch die Position mit dem kürzesten Namen, sondern schlichtweg die überzeugendste zum momentanen Zeitpunkt im Vergleich zu allen anderen Positionen. Wenn man so will, kommt dadurch auch eine Art eines methodologischen Prinzips zum Ausdruck: Es reicht für einen gegebenen Zweck zu einem gegebenen Zeitpunkt, eine Theorie, die mehr mit der Wirklichkeit übereinstimmt als ihre Konkurrenten, als wahr zu akzeptieren.

Zweitens, es ist auch das Ziel einer Diskussion, Anhänger des gegnerischen Lagers zum Überlaufen in das eigene Lager zu bewegen. Es reicht nicht bloß aus, seine Meinung nochmals kundzutun und die Meinung der Anhänger des eigenen Lagers nochmals zu bestärken. Es soll in der Diskussion eine Veränderung der Sichtweise und des - metaphysischen - Standpunktes geschehen. Die Akzeptanz einer neuen Position, die man zuvor noch nicht akzeptiert hat, ist dann gegeben, wenn man diese entsprechende Position auch für überzeugend hält. Damit ist der Weg frei, die neue Position als (vorläufig) wahr zu akzeptieren. Diese Diskussion trifft natürlich auch die Konklusion, welche die Wahrheit des Physikalismus als deren Inhalt hat: Er ist rationalerweise und zumindest vorläufig für wahr zu halten.

\subsubsection{Wann etwas überzeugend ist - Prämisse (2)}

Nun geht es um die Konkretisierung der Bedingungen, welche die Implikation zur überzeugendsten Position in der Philosophie des Geistes zulassen. Ich möchte damit beginnen, 
die wesentlichsten Argumentationsbereiche zu nennen, die typischerweise von beiden Lagern zur Rechtfertigung derer Positionen angesprochen werden. Die beiden Lager stellen einerseits die Physikalisten und andererseits die Dualisten dar. Einige von diesen Argumentationsbereichen sind uns in vorigen Kapiteln begegnet. Als Erstes sind es Kriterien wissenschaftlicher Theorien, bei denen beispielsweise die Einfachheit oder die Sparsamkeit als Vergleichsparameter herangezogen werden. Als Zweites sind es die Antworten, die auf einschlägige dualistische Gedankenexperimente gegeben werden. Als Drittes sind es Erkenntnisse aus den Naturwissenschaften, die nach und nach unser Wissen über den Zusammenhang zwischen unseren mentalen und physikalischen Vorgängen erweitern.

Jeder dieser drei Bereiche füllt Laufmeter philosophischer Bibliotheken. Jeder dieser drei Bereiche wird immer wieder in der gegenwärtigen Diskussion in der Philosophie des Geistes ins Treffen geführt, wenn für die eine oder andere Position argumentiert wird. Und jeder dieser drei Bereiche wird verwendet, um andere Philosophen für die eigene Position zu überzeugen und dadurch für das eigene Lager zu gewinnen. Üblicherweise wird in der Literatur vom jeweiligen Autor lediglich auf einen der drei Bereiche der Schwerpunkt gelegt und falls dieser Bereich den Ausschlag zugunsten des Physikalismus gibt, dann wird der Physikalismus als überzeugend und daraus folgend auch als wahr dargestellt. Ob nun die Überlegenheit in einem der drei Bereiche auf die anderen beiden Bereiche generalisierbar ist, ist jedoch eine offene Frage. Es wäre ganz klar eine gravierende Erhöhung der Überzeugungskraft des Physikalismus, wenn nicht nur in einem der klassischen Bereiche eine Überlegenheit gezeigt werden könnte, sondern in allen drei Bereichen. Aus diesem Grunde bildet die zweite Prämisse eine Konjunktion eben aus genau diesen drei klassischen Bereichen. Wenn nun alle drei Bereiche den Physikalismus gegenüber den konkurrierenden Positionen favorisierten, dann wäre der Physikalismus die überzeugendste Position in der Philosophie des Geistes.

\subsubsection{Die theoretische Überlegenheit des Physikalismus - Prämisse (3a)}

Die Prämisse (3a) sagt, dass der Physikalismus die Kriterien wissenschaftlicher Theorien besser erfüllt als der Dualismus. Um diese Prämisse plausibel zu machen, ziehe ich die Kriterien der Einfachheit und der Sparsamkeit heran, wie ich sie in Kapitel 4 definiert habe. Einfachheit bedeutet, dass eine gegebene Theorie T1 kognitiv zugänglicher oder rascher verstehbar oder übersichtlicher dargestellt oder eindeutiger erfassbar ist als eine Vergleichstheorie T2.

Sparsamkeit bedeutet, dass eine gegebene Theorie T1 i) weniger Entitäten, Eigenschaften, Relationen und dergleichen beinhaltet (= ontologische Sparsamkeit) und/oder ii) weniger Sätze, Wörter, Ausdrücke und dergleichen beinhaltet (= begriffliche Sparsamkeit) als eine Vergleichstheorie T2. Verglichen wird somit der Physikalismus als T1 mit dem Dualismus als T2. Ich möchte mit der Sparsamkeit beginnen, weil es der augenscheinlichste Unterschied zwischen beiden Lagern ist und im Grunde keine große Diskussion braucht. Der Physikalismus 
ist ontologisch sparsamer, weil er ohne die Existenz von fundamental mentalen Entitäten auskommt. Der Physikalismus ist auch begrifflich sparsamer, weil er eine Reduktion der mentalen Begriffe auf physikalische Begriffe beinhaltet oder die mentalen Begriffe durch physikalische Begriffe ersetzt werden. Hingegen benötigt der Dualismus weiterhin mentale Begriffe, um auf die nicht-reduzierbaren mentalen Entitäten zu referieren.

Hinsichtlich der Einfachheit möchte ich eine Reihe von Punkten bringen, die den Ausschlag für den Physikalismus geben und den Dualismus bei wohlwollender Interpretation anhält, weitere fragwürdige Annahmen vorzubringen. Der erste Punkt handelt davon, wie wir eigentlich Wissen über mentale Vorgänge erwerben bei Gültigkeit des Epiphänomenalismus, wenn die mentalen Entitäten eigentlich kausal impotent sind. Woher weiß ich, dass ich Schmerzen habe, und wie kann ich Wünsche und Absichten äußern, wenn ich davon eigentlich nichts wissen kann? Lösungsansätze gibt es dazu in der Literatur einige, alle haben gemeinsam, dass sie eine alternative Theorie zur standardmäßigen kausalen Theorie des Wissens vorlegen müssen. Bei der kausalen Theorie des Wissens weiß das Subjekt aufgrund des verursachenden Objektes selbst, sei es durch die Wahrnehmung eines Gegenstandes vor mir oder eines introspektiv zugänglichen Gefühlserlebnisses, dass es sich um genau dieses Objekt bzw. dieses Erlebnis handelt.

Chalmers $(1996,2003)$ beispielsweise löst dieses Problem so auf, indem er eine Bekanntschaftsthese anwendet (acquaintance theory). Chalmers ist nicht primär darauf gerichtet, über phänomenales Wissen zu sprechen, sondern legt seinen Fokus auf das Bilden von Glaubensannahmen über Qualia. Für ihn reicht das qualitative Erleben selbst, um auch Glaubensannahmen darüber bilden zu können:

[W]hat is it that justifies our beliefs about our experiences, if it is not a causal link to those experiences, and if it is not the mechanisms by which the beliefs are formed? I think the answer to this is clear: it is having the experiences that justifies the beliefs. (Chalmers, 1996, S. 182)

Chalmers (2003, S. 255-256) führt in späteren Arbeiten aus, dass Qualia für die Glaubensannahmen konstituierend sind und diese Art der Verbindung zwischen Qualia und Glaubensannahmen stärker ist als eine kausale Verbindung: "[l]f what I have said is correct, the connection between experience and phenomenal belief is tighter than any causal connection: it is constitution".

Nagasawas (2010, S. 50) Lösungsvorschlag hingegen ist die Einführung einer besonderen Identitätsthese, welche die Identität zwischen dem Wissen über das Roterlebnis und dem Roterlebnis selbst postuliert, wodurch das Subjekt S Wissen erwirbt: "The Identity Thesis: S's having qualia $q$ is identical to S's having relevant phenomenal knowledge about $q$ ". 
Nagasawas Ansatz hat die Konsequenz, dass jedes Quale im Prinzip ein kleines Paket an Wissen ist, dass es nur abzuholen gilt.

Vielleicht mag Chalmers es schaffen, dass ein bestimmtes Wissen über Qualia auf diesem Wege generiert werden kann, oder vielleicht mag Nagasawa es schaffen, dass sein Ansatz die Identität zwischen einem Quale und dem Wissen über dieses Quale herstellt. Jedenfalls wird eine Theorieentwicklung des Dualismus stetig voranschreiten. Welche Lösung auch immer schlagend wird, ich nehme es zum Zwecke des Arguments an, dass eine der beiden Lösungen schlagend wird, dann muss der Dualist sowohl in Chalmers' als auch in Nagasawas Fall eine nicht-standardmäßige Wissenstheorie annehmen. Es muss sozusagen ein besonderer Fall des Wissenserwerbes postuliert werden. Im Vergleich dazu kommt der Physikalist mit der weitläufig akzeptierten kausalen Wissenstheorie aus, weil das Wissen über ein Farberlebnis, das eigentlich nichts anderes als etwas Physikalisches ist, eben auch genau dadurch verursacht wird. Der Physikalismus ist somit einfacher als der Dualismus, weil er auf Altbekanntes zurückgreift. Darüber hinaus müssen Anhänger einer nicht-kausalen Wissenstheorie eine neue Art des Wissens, nämlich ein nicht-kausales Wissen, postulieren. Im Falle von Chalmers' Lösung muss zusätzlich eine neue fundamentale und gleichzeitig nichtkausale Relation angenommen werden. Von dieser Warte aus spricht die dualistische Lösung wiederum gegen eine ontologische Sparsamkeit und gibt nochmals den Ausschlag für den Physikalismus, weil dieser weder eine neue Wissensart noch eine neue fundamentale Relation in seine Ontologie aufnehmen muss ${ }^{51}$.

Der zweite Punkt betrifft viele weitere offene Fragen, denen sich der Dualismus stellen muss und die er womöglich auch lösen kann. Eine offene Frage ist beispielsweise diese: Wie kommt das Mentale in die Existenz und wie generiert etwas Gegebenes, nämlich das Physikalische, etwas komplett Verschiedenes zu sich selbst, nämlich das Mentale? Das Problem ist, dass eine Sache etwas intrinsisch Verschiedenes von sich aus produziert. Ich möchte dazu ein vereinfachtes Beispiel geben. Ein Licht einer bestimmten Wellenlänge fällt auf die Retina, der Reiz wird über den optischen Nerv zum visuellen Kortex weitergeleitet und das Subjekt hat ein Roterlebnis. Der Dualist muss behaupten, dass irgendwo auf diesem Wege das intrinsisch mentale Roterlebnis sich aus den intrinsisch physikalischen Reizübertragungen von der Retina bis zum visuellen Kortex ergibt. Der Dualist postuliert womöglich eine bestimmte psychophysische Gesetzmäßigkeit, zu deren Kenntnis er durch Introspektion gelangt ist, um

\footnotetext{
${ }^{51}$ Falls nun doch, aus irgendwelchen Gründen auch immer, ein interaktionistischer Dualismus vertreten wird, ergibt sich ein anderes Problem. Es ist das Problem der Verletzung der kausalen Geschlossenheit des Physikalischen. Karl Popper löst das Interaktionsproblem mit der Einführung seiner Theorie der drei Welten (Popper, 1994). In Welt 1 sind alle physikalischen Entitäten (z.B. das Gehirn), in Welt 2 alle psychischen Entitäten (z.B. Qualia) und in Welt 3 die Produkte des menschlichen Geistes (z.B. wissenschaftliche Theorien). Die klassische Körper-Geist-Interaktion geschieht nun zwischen Welt 1 und Welt 2. Doch was uns Popper schuldig bleibt, ist der genaue Mechanismus, wie die Interaktion von verschiedenen Welten konkret geschieht. Ähnlich der Ansatz von Eccles (1989), in dem das Mentale im synaptischen Spalt durch quantenmechanische Gesetzmäßigkeiten eine Interaktion mit der physischen Welt eingeht. Auch hier fehlen die konkreten Details, wie solch eine Interaktion von zwei wesensverschiedenen Entitäten möglich sein kann.
} 
diesen Vorgang des Entstehens noch genauer zu erklären. Vielleicht beruft sich der Dualist aber auch auf die Intuition, dass eben das Entstehen des Mentalen einfach passiert und der genaue Prozess nicht konkreter beschrieben werden kann.

Dies führt zur nächsten Frage: Wo und in welcher Art und Weise existiert das Mentale, sobald es entstanden ist? Die Standardsicht des Dualisten ist, dass das Mentale räumlich nicht lokalisiert ist und somit auch streng genommen weder im Gehirn als Gesamtes oder spezifisch am Entstehungsort am Wege der Reizübertragungskette ist. Der Dualist könnte beispielsweise postulieren, dass das Mentale außerhalb des Raumes existiert, seit Einstein eher außerhalb der Raumzeit, und mit einer bestimmten psychophysischen Relation mit dem Subjekt verbunden ist. Das führt natürlich wieder zu weiteren Fragen, wie dieser: Wohin geht das konkrete Vorkommnis des Mentalen, wenn wir den konkreten Gedanken oder das konkrete Gefühl nicht mehr haben? Der Dualist könnte hier erwidern, dass sich das Mentale entweder auflöst oder sich einfach wieder in seine Existenzweise außerhalb von Raum und Zeit zurückzieht und als existierende Entität weiterbesteht. Hier ist zu erwarten, dass der Dualismus immer mehr Fortschritte in seiner Theorienbildung erfahren wird und die obigen offenen Fragen vielleicht sogar beantwortet werden. Der Preis dieser Fragebeantwortung, so fürchte ich, wird sein, dass sie die dualistischen Theorien immer komplexer und weniger einfacher machen ${ }^{52}$.

Es geht hier also nicht darum, dass das Mentale beispielsweise nicht-räumlich ist und sich deswegen der Physikalist daran stößt. Physikalisten akzeptieren typischerweise nichträumliche Entitäten wie beispielsweise Sachverhalte oder Zahlen. Es geht vielmehr darum, dass durch eine Nicht-Räumlichkeit des Mentalen ein hoher Komplexitätsgrad an dualistischen Erklärungsmodelle einhergeht, auf das der Physikalist entgegnet, dass es eigentlich nicht notwendig sei. Es ist deshalb nicht notwendig, weil es weniger komplexe Erklärungsmodelle in den Händen des Physikalisten gibt. Der springende Punkt ist hier auch nicht, ob der Dualismus diese Fragen tatsächlich beantworten kann, sondern ob die dualistische Lösung einfacher ist als die physikalische Lösung. Zum Zwecke des Arguments wird angenommen, dass eine befriedigende dualistische Lösung vorgelegt werden kann, doch diese Lösung wird mit vielen zusätzlichen Annahmen zurechtkommen müssen. Mögliche dualistische Antworten auf die obigen Fragen sollen lediglich verdeutlichen, wie vorgegangen werden könnte und welche Konsequenzen dieses Vorgehen in sich birgt. Vergleichen wir die potentiellen dualistischen Lösungen mit der Antwort eines Physikalisten. Der Physikalist behauptet, dass es nur die Reizübertragung gibt. Das Farberlebnis ist genau an einem Punkt dieser Reizübertragungskette festzumachen oder systemisch in Bezug auf mehrere Gehirnstrukturen festzumachen. Das Farberlebnis ist damit auch eindeutig räumlich lokalisiert, entweder in einer Gehirnstruktur oder

\footnotetext{
${ }^{52}$ Falls ein interaktionstischer Dualismus vertreten wird, ergeben sich auch hier dieselben Probleme. Einzig allein das Problem der räumlichen Lokalisierung stellt sich etwas verschieden dar, da im konkreten Moment der Körper-GeistInteraktion sinnvollerweise so etwas wie ein räumlicher Kontaktpunkt zwischen dem Physikalischen und dem Mentalen vorhanden ist. Doch zu den Zeiten, wo das Mentale nicht mit dem Physikalischen interagiert, ist das Gesagte mutatis mutandis auf den interaktionistischen Dualismus übertragbar.
} 
im systemischen Bezug mehrerer Gehirnstrukturen. Das Farberlebnis verschwindet zeitlich auch wieder, sobald etwas anderes ins Bewusstsein tritt, genauso wie diese bestimmte Reizübertragung nach einer gewissen Zeit abbricht, sobald eine andere Gehirnstruktur aktiviert wird. Mit diesen beiden Versionen der Geschichte eines Roterlebnisses ist die physikalistische Geschichte einfacher als die dualistische Geschichte. Zusammengefasst erfüllt der Physikalismus die Kriterien wissenschaftlicher Theorien, so wie ich sie in dieser Arbeit eingeführt habe, besser als der Dualismus.

\subsubsection{Apathische Mary - Prämisse (3b)}

Die Prämisse (3b) sagt, dass der Physikalismus dualistische Gedankenexperimente besser erklärt als der Dualismus selbst. Um diese Prämisse plausibel zu machen, möchte ich den Physikalismus und den Dualismus an klassischen dualistischen Gedankenexperimenten testen, welche die Falschheit des Physikalismus als deren Konklusion haben. Dabei handelt es sich im großen Maße um eine praktische und konkrete Anwendung der Erkenntnisse, die zuvor in der Diskussion der Prämisse (3a) erreicht wurden. Das bekannteste und wohl meistdiskutierteste ist das Argument des unvollständigen Wissens mit der Hauptprotagonistin Mary (Jackson, 1982, 1986). Jacksons Argument ist natürlich nicht das einzige dualistische Argument, doch ich diskutiere in diesem Kapitel ausschließlich Jacksons Argument und der Grund ist lediglich ein dialektischer. Das Argument des unvollständigen Wissens ist so aufgebaut, dass es eine immense intuitive Kraft hat. Es ist ein Beitrag, der packend und lebhaft ist. Dadurch ist Jacksons Argument die überzeugendste Version eines dualistischen Arguments. Aus diesem Grunde sehe ich Jacksons Argument nicht nur als das überzeugendste dualistische Argument an, sondern auch als die letzte Verteidigungslinie für das dualistische Lager. Denn wenn Jacksons Argument überzeugender ist als jedes andere dualistische Argument und der Physikalismus Jacksons Argument besser erklärt als der Dualismus, dann erklärt auch der Physikalismus jedes andere dualistische Argument besser als der Dualismus selbst ${ }^{53}$.

Im Allgemeinen ist die Frage diejenige, wie die Situation zu interpretieren ist, in der sich Mary nach Verlassen ihres schwarz-weißen Zimmers befindet. Die dualistische Interpretation ist überraschenderweise spärlich im konkreten Inhalt, wie die Situation über die Postulierung hinaus, dass das Mentale fundamental sei, erklärt wird. Oftmals wird durch die scheinbare

\footnotetext{
${ }^{53}$ An dieser Stelle sei angemerkt, dass viele dualistische Gedankenexperimente mit aller Wahrscheinlichkeit miteinander zusammenhängen. Beispielsweise argumentiert Stoljar (2006), dass Jacksons Mary-Argument und Chalmers' Zombie-Argument eigentlich dieselbe logische Struktur haben und ineinander überführt werden können. Darüber hinaus weise ich darauf hin, dass mit großer intuitiver Kraft auch argumentiert werden könnte, dass im Argument des unvollständigen Wissens eine Erklärungslücke zwischen dem physikalischen und dem phänomenalen Wissen impliziert wird (vgl. Levine), genauso wie die Erste-Person-Perspektive von Mary nach dem Verlassen ihres schwarz-weißen Zimmers ein Dreh- und Angelpunkt darstellt (vgl. Nagel). Zu guter Letzt bildet Mary beim ersten Anblick einer roten Rose einen neuen Begriff, nämlich einen phänomenalen Rot-Begriff, der ohne das vorangehende Erlebnis nicht gebildet werden könnte (vgl. Nida-Rümelin). Für den vorliegenden Zweck habe ich bloß auf die möglichen Zusammenhänge zwischen Jacksons Argument und den restlichen dualistischen Argumenten hingedeutet, um die zentrale Rolle von Jacksons Gedankenexperiment von diesem Blickwinkel aus zu unterstreichen. Vielleicht ist es am Ende des Tages so, dass alle dualistischen Argumente so etwas wie eine Fläche eines Würfels darstellen. Im Grunde sagen alle dasselbe aus, lediglich in einem anderen verbalen Gewand.
} 
Widerlegung des Physikalismus bloß der Dualismus hergestellt, doch was dann? Es erinnert an einen Liebesfilm, bei dem in den letzten Spielminuten doch noch das Paar zusammenkommt und dann ist der Film zu Ende. Aber was geschieht danach im Alltag des Liebespaares, wie wird der erste Streit überwunden, wer trägt den Müll raus? Pointiert dargestellt ist so auch die Situation in der dualistischen Literatur. Glücklicherweise wurden einige Lücken seit Veröffentlichung von Jacksons Argument mit Inhalt aufgefüllt und der Dualismus gewann immer mehr an Elaboriertheit, Überzeugungskraft und deckte verschiedene Themenbereiche detailreicher ab. Diese Erhöhung des Entwicklungsgrades des Dualismus führt schließlich zu einer Erklärung, die Marys Wissenszuwachs erklärt: Sie ist überrascht nach dem Verlassen ihres schwarz-weißen Zimmers. Durch das erste Sehen einer roten Rose tritt die Eigenschaft eines Rot-Quales in die Existenz, was mit fundamentalen psychophysischen Gesetzen mit den physikalischen Vorgängen einer Rotwahrnehmung zusammenhängt. Indem das Rot-Quale selbst das neue faktische Wissen darstellt (Nagasawas Ansatz) oder indem durch die Bekanntschaft mit dem Rot-Quale alleine ein nicht-kausales Wissen gebildet wurde (Chalmers' Ansatz), ist die epiphänomenale Natur bewahrt. Anschließend bildet Mary eine Erinnerung an dieses bewusste Erlebnis und kann durch ihr Erinnern daran auch immer wieder das Rot-Quale neuerlich in die Existenz treten lassen.

So weit, so gut, die grundlegenden Pfeiler der Erklärung sind aufgestellt. Doch wie hoch ist die Erklärungskraft des dualistischen Ansatzes? Um das genauer abzuschätzen, will ich diejenigen Punkte bringen, die üblicherweise nicht in solche Erklärungen aufgenommen werden. Der Dualist muss nämlich noch weiter die Situation beschreiben, indem er beispielsweise postulieren muss, dass das Mentale als etwas real Existierendes nie aus einer Dritte-PersonPerspektive wahrgenommen werden kann und sogar aus der Erste-Person-Perspektive im herkömmlichen Sinne weder gesehen, angegriffen oder örtlich festgemacht werden kann. Weiters muss der Dualist sagen, dass die psychophysischen Gesetze im herkömmlichen Sinne weder überprüfbar oder falsifizierbar noch explizierbar sind. Nichtsdestotrotz muss der Dualist zur Erkenntnis kommen, dass das Mentale als eine Eigenschaft zum Physikalischen hinzukommt. Die konkreten Details, wie zu dieser Erkenntnis gelangt wird, sind wiederum der Introspektion jedes einzelnen Menschen überlassen. Doch welche Regeln einer guten wissenschaftlichen Introspektionspraxis gelten und welche Qualitätsstandards müssen dabei eingehalten werden, um das nächste Audit zu überstehen? Oder als letztes Beispiel muss der Dualist behaupten, dass das Mentale entweder in einer besonderen Sphäre innerhalb unserer raumzeitlichen physikalischen Welt oder in einer Sphäre außerhalb unserer Raumzeit existiert und eben durch die psychophysischen Gesetze, die zwei völlig verschiedene Sphären miteinander verbinden, kurzzeitig in unsere gewöhnliche physikalische Sphäre geholt wird. Anschließend wird sozusagen eine Standleitung aus unserem Hippocampus, der für unser 
Gedächtnis zuständig ist, in diese mentale Sphäre gelegt, wenn ein Roterlebnis wieder erinnert wird $^{54}$.

In dieser Art und Weise muss der Dualist die Lücken füllen, um den Sachverhalt des Wissenserwerbes von Mary gut erklären zu können. Von einer anfänglichen dualistischen Intuition sind wir bei genauerer Betrachtung zum Ergebnis gelangt, dass das zu Erklärende nur durch weitere zu erklärende Postulate ersetzt wird. Hier ist auch zu erwarten, dass mit Fortschritt der dualistischen Theoriebildung noch weitere Annahmen, Postulate und Erklärungen hinzugefügt werden, doch ich fürchte, dass die prinzipielle Aussage gleich bleibt: Dualistische Theorien werfen immer mehr zu erklärende Dinge auf, die nicht durch noch so komplexe Annahmen und Theorienbestandteile vollständig beantwortet werden. Vergleichen wir die dualistische mit der physikalistischen Interpretation des Mary-Falles. Auch hier gewann im Zuge des Fortschrittes der Naturwissenschaften der Physikalismus an Elaboriertheit, Überzeugungskraft und deckte verschiedene Themenbereiche detailreicher ab. Dies wird bis zur vollendeten Physik auch so weitergehen und zu folgender Lösung führen. Mary wird teilnahmslos und abgestumpft, schlichtweg apathisch, auf das Wahrnehmen ihrer ersten roten Rose reagieren und sagen: "Genau so habe ich mir vorgestellt, wie es sein wird, Rot zu sehen. Ich habe bereits in meinem schwarz-weißen Zimmer aus den physikalischen Fakten abgeleitet, wie es ist, Rot zu sehen. Durch das letztendliche Sehen einer roten Rose habe ich nichts dazugelernt". Die einzige Geschichte, die Mary wissen muss, ist die Geschichte des Auftretens einer bestimmten Wellenlänge auf die Retina und die Reizweiterleitung über den optischen Nerv, bis zum visuellen Zentrum des Gehirns. Das Rot-Quale ist nichts anderes als eben genau dieser physikalische Prozess. Eine Erinnerung an ein Rot-Quale in diesem bestimmten Moment wird anschließend aufgrund der neuronalen Plastizität des Hippocampus gebildet.

Alles, was es zur Erklärung braucht, sind altbekannte und bewährte Erkenntnisse. Es sind physikalische Gesetze der Reizweiterleitung visueller Eindrücke, es sind beobachtbare physikalische Prozesse in unseren Gehirnen, die prinzipiell falsifizierbar sind und einschlägigen Qualitätsstandards einer guten wissenschaftlichen Praxis entsprechen, und es ist das Verorten der vermeintlichen mentalen Sphäre in unsere herkömmliche physikalische Sphäre. Bei genauerem Hinschauen ergeben sich auch hier noch Fragen, doch diese Fragen sind prinzipiell beantwortbar und werden sich auflösen, wie sich vergleichbare Fragen in der Vergangenheit aufgelöst haben. Es ist die naturwissenschaftliche Methodik, welche diese Erkenntnisse hervorbringt und zum Zeitpunkt einer vollendeten Naturwissenschaft auch alles hervorgebracht haben wird, was es zum Hervorbringen gibt. Eine hohe Erklärungskraft ist somit nicht bloß dem ersten Anscheine gegeben, sondern auch bei genauerem Nachhaken, denn der Physikalist behandelt den Mary-Fall bei jeder Stufe nachvollziehbar und völlig explizierbar.

${ }^{54}$ Auch für Anhänger eines interaktionistischen Dualismus ergeben sich dieselben Probleme. 
Zusammengefasst erklärt der Physikalismus Jacksons Argument, und per Transitivität klassische dualistische Gedankenexperimente insgesamt, besser als der Dualismus selbst.

\subsubsection{Die empirische Unterstützung des Physikalismus - Prämisse (3c)}

Die Prämisse (3c) sagt, dass der Physikalismus durch wissenschaftliche Erkenntnisse besser gestützt wird als der Dualismus. Um diese Prämisse plausibel zu machen, möchte ich den gegenwärtigen Stand der Naturwissenschaften kurz darlegen und zeigen, wie der subjektive Erlebnisinhalt von Emotionen bereits jetzt in vielerlei Hinsicht erfasst werden kann. Wenn man so will, ist dieser Teil der dritten Prämisse im großen Maße eine wissenschaftliche Fundierung der Erkenntnisse aus der Diskussion der Prämisse (3b). Hier geht es nicht darum, aufgrund der empirischen Ergebnisse auf die Identität zwischen dem Mentalen und dem Physikalischen abduktiv zu schließen, sondern darum, aufgrund der Ergebnisse den Vorzug in die Richtung des Physikalismus zu geben. Bisherige psychologische Ergebnisse zeigen, dass wir Menschen immer noch an das Spezielle des Menschseins glauben wollen, trotz vieler gegenläufiger Erkenntnisse aus den Naturwissenschaften (Mogi, 2013; Gottlieb \& Lombrozo, 2018). Dass diese letzte Bastion der Besonderheit dennoch durch die Naturwissenschaften gestürmt werden kann (Dennett, 1988), möchte ich nachfolgend zeigen.

Vielleicht möge man mir an dieser Stelle bereits vorwerfen, dass die Naturwissenschaften sowieso immer für den Physikalismus sprechen und nie für den Dualismus, weil die Naturwissenschaften nie nach mentalen Entitäten suchen, und aufgrund dessen das Ergebnis der Analyse bereits vorweggenommen ist. Dazu möchte ich mit einer Unterscheidung zwischen dem methodologischen und dem ontologischen Naturalismus entgegnen. Der methodologische Naturalismus hat die Aufgabe, zu zeigen, wie die physikalischen Entitäten gesetzesmäßig und kausal miteinander verwoben sind. Der ontologische Naturalismus hingegen hat die Aufgabe, zu zeigen, was tatsächlich als etwas Physikalisches existiert. Beide Arten des Naturalismus sind nicht äquivalent. So ist es möglich, dass die Naturwissenschaften methodisch nicht explizit nach etwas fundamental Mentalem suchen, obwohl es ontologisch tatsächlich existiert. Ob etwas Mentales tatsächlich existiert, ist letztendlich eine ontologische Frage und keine methodische Frage.

Es kann sehr wohl sein, wie unwahrscheinlich es auch sein möge, dass zur Erklärung einer physikalischen Beobachtung keine physikalischen Gründe gefunden werden können. Falls nun alle empirischen Ergebnisse in diejenige Richtung gehen, dass jegliche physikalischen Entitäten ausgeschlossen werden und mentale Entitäten für die Erklärung der Beobachtungen angenommen werden müssen, warum auch immer das der Fall ist, dann würde das für den Dualismus sprechen. Ich nehme an dieser Stelle den Hinweis von Bird (2007), um die Existenz von mentalen Gründen auf Basis des Schlusses zur einzigen Erklärung (inference to the only explanation) zu begründen. Genauso wie Sherlock Holmes sagen würde: "Eliminate the 
impossible, and whatever remains, however improbable, must be the truth" (Bird, 2007, S. 425). Alles in allem können in dieser Art und Weise die Naturwissenschaften, völlig zufällig und unbeabsichtigt, fundamental Mentales in unserer Welt indirekt und durch einen Schluss zur einzigen Erklärung aufzeigen.

Ich möchte nachfolgend das Erlebnis von Furcht heranziehen, um meinen Punkt zu erläutern. Ein ähnliches Konstrukt ist das der Angst, das sich von dem der Furcht geringfügig, aber wesentlich, unterscheidet. Beide Ausdrücke werden umgangssprachlich als synonym aufgefasst, doch ist der wesentliche Unterschied einerseits der, dass sich "Angst" auf eine eher ungewisse Befürchtung von potentialen Gefahren richtet (z.B. Angst, dass bald etwas Schreckliches passieren wird) und "Furcht" sich auf eine identifizierbare und konkrete Gefahr bezieht (z.B. Furcht vor einer Schlange direkt vor mir), und andererseits der, dass beide emotionalen Qualitäten auch eine andere neurobiologische Basis haben (Knight \& Depue, 2019). Furcht ist ein emotionales Erlebnis, das wir alle kennen und das ebenso in der Tierwelt vorhanden ist. So wie Tiere in Furchtsituationen typischerweise mit zweierlei Reaktion kontern, nämlich entweder mit Flucht oder mit Angriff, reagieren auch Menschen in gleicher Art und Weise, obgleich das Verhaltensrepertoire deutlich breiter gefächert ist. Auf der Verhaltensebene gibt es für uns Menschen bereits eine Vielzahl weiterer Korrelate als zu flüchten oder anzugreifen, wie beispielsweise eine robuste selektive Aufmerksamkeitszuwendung auf die bedrohlichen Reize bei Furcht (Bar-Haim, 2007; MacLeod et al., 2019) und verwandten Emotionen wie bei Sorgen (Gole et al., 2012). Auf physiologischer Ebene hängt Furcht mit körperlichen Reaktionen über unser unwillkürliches, autonomes Nervensystem zusammen. Darunter finden sich beispielsweise eine Verminderung in der Herzratenvariabilität während des Ruhezustandes (Chalmers et al., 2016), eine Erhöhung der Hautleitfähigkeit (Rosebrock et al., 2016), eine Erhöhung des Muskeltonus (Pluess et al., 2009), eine Erhöhung der Atemfrequenz (Gomez et al., 2005) und eine Erhöhung des Blutdruckes (Sacchetti et al., 2017). Eine Aktivierung der Stressachse (Hypothalamus-Hypophyse-Nebennierenrinde-Achse) ist jedoch nur bei einer antizipatorischen und generalisierten Angst zu finden, jedoch nicht bei Furcht (Graeff \& Junior, 2010).

Diese Bereiche des Verhaltens und des autonomen Nervensystems sind zentralnervös durch unser Gehirn gesteuert und so ist es nicht verwunderlich, dass auch auf neurobiologischer Ebene Furcht beschreibbar ist. Beispielsweise wissen wir von Studien aus dem Bereich der Elektroenzephalographie (EEG), dass bei Betrachtung von aversiven Bildern Furcht mit einer deutlichen Erhöhung der Aktivität um die 300 ms und auch danach bis ca.1500 ms nach der Bildbetrachtung einhergeht, wobei dieser Effekt deutlicher bei Personen mit Angsterkrankungen ist und durch eine Verhaltenstherapie normalisiert werden kann (Leutgeb, 2009). Ähnliche Ergebnisse liefern Studien zu einer differenziellen Modulierung von noch früheren EEGKomponenten zwischen 70 ms und 200 ms, die ebenso durch eine gezielte Verhaltenstherapie auf ein normales Niveau gebracht werden kann (Cao et al., 2017). Als Grundlage des EEGs 
dient die elektrische Aktivität in unserem Gehirn, bei dem je nach Neuronentyp das elektrische Signal direkt von einer Synapse zur nächsten übertragen wird (gap junctions) oder mit einem bestimmten Austausch von lonen zwischen dem intrazellulären und dem extrazellulären Raum des Neurons sowie einer damit verbundenen Änderung der Ladung der Zellmembran das elektrische Signal an der Synapse in ein chemisches Signal umgewandelt wird (Zschoke, 2002; Olejniczak, 2006). Im letzteren Fall besteht das chemische Signal aus Neurotransmittern, die an der nächsten Synapse an Rezeptoren andocken und dadurch wiederum durch einen bestimmten Austausch von lonen zwischen dem intrazellulären und dem extrazellulären Raum eine Ladungsänderung der Zellmembran auslösen und das chemische Signal wieder in ein elektrisches Signal umgewandelt wird.

Aus dem bildgebenden Bereich, vor allem der funktionalen Magnetresonanztomographie (fMRT) und der Positronenemissionstomographie (PET) wissen wir weiters, dass Furcht sowohl subkortikale als auch kortikale Areale involviert. Während die PET seit Mitte der 90er-Jahre in der Bildgebung immer mehr an Bedeutung verliert, gewinnt die fMRT mehr an Bedeutung. Die Grundlage einer fMRT ist die Registrierung einer Veränderung des Sauerstoffgehaltes im Blut, denn bei einer erhöhten Aktivität in einem Gehirnareal erhöht sich auch die Blutzufuhr, was wiederum das Level an paramagnetischem, desoxygeniertem Hämoglobin im Blut reduziert (sog. BOLD-Kontrast: blood ogygenation level dependent; Logothetis, 2002; Buxton, 2013). Diese Veränderung wird durch eine fMRT in einem starken Magnetfeld aufgezeichnet und erlaubt eine genaue Lokalisierung der Gehirnaktivität, die mit mentalen Vorgängen einhergehen.

Es zeigte sich sowohl bei Angststörungspatienten als auch bei gesunden Kontrollprobanden eine höhere Aktivität in der Amygdala (subkortikaler Bereich) und der Insula (kortikaler Bereich), wobei dieser Effekt noch stärker in der Gruppe der Angststörungspatienten ist (Etkin \& Wager, 2007). Insgesamt zeigt sich in der neurowissenschaftlichen Literatur konsistent eine Aktivierung der Amygdala (subkortikaler Bereich), der Insula (kortikaler Bereich), des anteriorien zingulären Gyrus (ACC; subkortikaler Bereich) und von Teilen des präfrontalen Kortex (PFC; kortikaler Bereich) bei Furcht (Taylor \& Whalen, 2015). Dabei betonen einige Autoren die Wichtigkeit einer bestimmten Art von Neuronen mit nicht-myelinisierten Axonen, die in der Amygdala und der Insula vorkommen, und eine Rolle im Entstehen von Bewusstsein spielen würden (Damasio \& Carvalho, 2013). Andere wiederum kehren ausschließlich die Rolle subkortikaler Bereiche hervor (Panksepp et al. 2017). Doch die Bedeutung dieser empirischen Befunde ist nicht in allen Bereichen eindeutig, dadurch verwundert es nicht, dass es verschiedene Interpretationen der konkreten Rollen dieser neuronalen Korrelate gibt.

So ist unter anderem die konkrete Rolle des PFC umstritten (Knotts et al., 2018). Es könnte sein, dass der PFC indirekt für subjektive Erlebnisse zuständig ist, beispielsweise durch seine Verbindungen mit unspezifischen Kernen im Thalamus (León-Domínguez \& León-Carrión, 2019) und durch die Möglichkeit, aufgrund der funktionalen Koppelung an die Amygdala deren Aktivität zu attenuieren (Kim et al., 2011). Andere Autoren meinen, dass weder dem PFC noch 
subkortikalen Arealen eine direkte Rolle zukommt, sondern Bewusstsein in einer posterior heißen Zone (posterior hot zone) entsteht, nämlich durch ein Zusammenwirken von parietalen, okzipitalen und temporalen Regionen (Koch et al., 2016). Ähnlich argumentieren LeDoux und Brown (2017), die für ein Furchterlebnis ähnliche kortikale Areale verantwortlich sehen, ohne direkte Involvierung subkortikaler Strukturen. Hinsichtlich der Rolle der Amygdala zeigt sich auch in anderen Bereichen, dass sie nicht notwendig für Furchterlebnisse ist (z.B. Anderson \& Phelps, 2002; Feinstein et al., 2013).

Weitere Hinweise zur neuronalen Basis von subjektiven Erlebnissen abseits der Furcht kommen unter anderem von Mazzi et al. (2017). Mazzi und Kollegen haben gezeigt, dass ein Erzeugen eines Magnetfeldes (sog. transkraniale Magnetstimulation, TMS) über okzipitalen und parietalen Regionen ca. 70 ms nach Applikation der TMS ein visuelles Erlebnis bei einem Probanden mit beidseitiger Schädigung des visuellen Kortex hervorgerufen werden kann. Yih et al. (2019) konnten hingegen durch eine intrakraniale Stimulation von Neuronenpopulationen in der Insula, im ACC und im orbitofrontalen Kortex (OFC, kortikaler Bereich) subjektive Erlebnisinhalte generieren. Dabei wurde das subjektive Erlebnis umso stärker empfunden, je stärker auch die elektrische Stimulation war. Schließlich gibt es bereits erste Erfolge, den konkreten Erlebnisinhalt auf Basis der neuronalen Aktivitäten durch einen Algorithmus vorherzusagen (Kassam et al., 2013).

Die wesentlichste Erkenntnis daraus für das Bewusstseinsproblem ist die, dass im Zuge der vielen funktionalen und strukturellen Verbindungen im menschlichen Gehirn, aber auch den elektrischen, chemischen und physikalischen Gegebenheiten, keine fundamental mentalen Entitäten auftauchen. Eine dualistische Interpretation der Erkenntnislage wird immer schwieriger durchzuführen, denn wie passen Qualia in das Bild hinein? In den gesamten Naturwissenschaften bedarf es ausschließlich der Geschichte der empirischen Ergebnisse, um ein Verständnis unseres vermeintlich mentalen Lebens zu erlangen. Insgesamt ist es das Ergebnis unserer Entwicklungsgeschichte, dass sich das Gehirn genau so entwickelt hat, mit all den Strukturen und Funktionen, die wir immer genauer messen können und dabei subjektive Erlebnisse nicht vergessen oder gar auslassen. Die berühmte Erklärungslücke von Levine schließt sich und Chalmers' schwieriges Problem des Bewusstseins kollabiert in die einfachen Probleme: Physikalische Erkenntnisse sind ein und dasselbe wie mentale Erkenntnisse. Die genaue Reduktionsbasis für das Bewusstsein ist zum gegenwärtigen Stand der Forschung noch nicht eindeutig identifiziert, obgleich bereits einige Kandidatentheorien vorhanden sind, sei es nun die Theorie des globalen Arbeitsspeichers (Baars, 2019) oder auch die Theorie der integrierten Information (Tononi et al., 2016), die jede für sich genommen weitere Forschungsvorhaben generieren, um das konkrete Bild der naturwissenschaftlichen Fundierung immer besser ausmalen zu können. In der Entwicklung der gegenwärtigen Physik zu einer vollendeten Physik wird das Bild kräftig gemalt sein, mit satten Farben und einer meisterhaften 
Pinselführung. Zusammengefasst wird der Physikalismus durch wissenschaftliche Erkenntnisse besser als der Dualismus gestützt ${ }^{55}$.

\subsection{Der Einwand monistischer Gegenpositionen}

Der Lieblingsgegner des Physikalisten ist der Dualist. Durch die erfolgreiche Verteidigung des generalistischen Arguments für den Physikalismus ist der Dualismus besiegt, doch leider scheint der Weg für den Physikalismus noch nicht vollständig geebnet zu sein. Wir sind noch nicht vollständig am Ziel angelangt, so könnte ein Einwand gegen mich lauten, ohne auch die letzte Hürde zu überwinden. Dabei handelt es sich um konkurrierende monistische Positionen, die etwas anderes als das Physikalische als grundlegende Entität allen Seienden annehmen. Aus der Falschheit des Physikalismus folgt nicht notwendigerweise der Dualismus, genauso wie der Dualist nicht den einzigen Gegner des Physikalismus darstellt. Ich möchte nachfolgend den Idealismus, den neutralen Monismus und als überschneidende Position zwischen den beiden den Panprotopsychismus behandeln. Alle drei monistischen Positionen sind sich mit dem Physikalismus einig, dass es nur eine einzige grundlegende, natürliche Art von etwas Seiendem gibt. Alle drei sind sich jedoch auch mit dem Dualismus einig, dass es nicht das Physikalische ist.

Der Grund, warum diese alternativen monistischen Positionen noch eine Hürde darstellen, so geht der Einwand weiter, spiegelt sich in den Prämissen (3a) bis (3c) wider. Hinsichtlich der Prämisse (3a) erfüllt der Physikalismus die Kriterien wissenschaftlicher Theorien nicht besser als die alternativen monistischen Positionen und ist deshalb nicht aufgrund theoretischer Überlegungen vorzuziehen. In Bezug auf die Kriterien der Sparsamkeit und der Einfachheit sind alle monistischen Positionen als gleichrangig zu bewerten, weil es nur eine ontologische Kategorie mit dazugehörigen Begriffsgattungen gibt und weil sie alle im selben Maß kognitiv zugänglich oder rasch verstehbar oder übersichtlich dargestellt oder eindeutig erfassbar sind. Hinsichtlich der Prämisse (3b) erklärt der Physikalismus klassische dualistische Gedankenexperimente gleich gut wie seine monistischen Rivalen, denn alle klassischen vermeintlich physikalischen Gesetze halten und der Wissenserwerb von Jacksons Mary kann zufriedenstellend erklärt werden. Hinsichtlich der Prämisse (3c) ergibt sich ein ähnliches Bild, nämlich jenes, dass die Gesamtheit der Naturwissenschaften sowohl den Physikalismus als auch die anderen monistischen Positionen gleich gut unterstützt, denn auch hier bleiben alle vermeintlich physikalischen Erkenntnisse aufrecht.

Indem ein Monismus vertreten wird, ist damit auch gesichert, dass das Mentale und das Physikalische Teil derselben Welt sind und sich viele Probleme des klassischen Leib-SeeleProblems erst gar nicht ergeben. Das trifft sowohl den Physikalismus als auch die drei anderen

55 Diese Erläuterungen treffen in gleicher Stärke ebenfalls auch den interaktionistischen Dualismus zu. 
monistischen Rivalen. Beispielsweise wäre es hinsichtlich des Problems der mentalen Kausalität lediglich die Kausalität von etwas Mentalem zu etwas anderem Mentalen (bei Gültigkeit des Idealismus) oder von etwas Neutralem wieder zu etwas anderem Neutralen (bei Gültigkeit des neutralen Monismus) oder auch von etwas Panprotopsychischem zu etwas anderem Panprotopsychischen (bei Gültigkeit des Panprotopsychismus). Bevor ich diese letzte Hürde nehme und gegen die drei Konkurrenten argumentiere, betrachten wir diese zunächst etwas mehr im Detail.

\subsubsection{Der Idealismus}

Der Idealismus ist uns bereits in Kapitel 2 begegnet, in dem ich inn als "starken Mentalismus" bezeichnet habe. Die Grundthese des Idealismus ist, dass alles mental sei. Bäume, Steine, Elektronen und Quarks scheinen lediglich physikalisch zu sein, doch lassen sie sich ohne Rest auf etwas Mentales reduzieren. Damit ist der Idealismus alles das, was auch der Physikalismus ist, nur mit einem anderen Vorzeichen. Was es nun bedeutet, wenn etwas mental ist, wird durch Bezug auf Intentionalität und Qualia definiert. Klassischerweise wurde eine radikale Form des Idealismus von Berkeley (1734) vertreten, bei der lediglich das eigene Bewusstsein und Gott existieren, aber nichts anderes. Diese strenge Spielart des Idealismus hat nicht viel fruchtbaren Boden in der gegenwärtigen metaphysischen Diskussion gefunden und der Idealismus wurde fälschlicherweise mit der Berkeley'schen Spielart gleichgesetzt und dadurch vorschnell nicht mehr weiter beachtet. Taylor (2018, erster Absatz) hat in seiner Rezension von Goldschmidt und Pearce (2017) den momentanen Stand des Idealismus so beschrieben:

Nowadays, however, the bulwarks of idealism are largely abandoned. Few defend this view, and fewer still are willing to take the time to consider its claims seriously. Materialism and dualism dominate the philosophical landscape.

Die spezifischen Details idealistischer Strömungen variieren (siehe die Übersicht von Chalmers, 2019) und seit Berkeley hat sich vieles getan, denn neue Vertreter eines modernen Idealismus sind hervorgetreten (siehe den Sammelband von Goldschmidt \& Pearce, 2017; aber auch Autoren abseits dieses Sammelbandes wie Kastrup, 2007; Foster, 2008; Goff, 2017). Insgesamt ist der Idealismus wohl der bekannteste Monismus neben dem Physikalismus.

\subsubsection{Der neutrale Monismus}

Der neutrale Monismus ist unbekannter als der Idealismus, doch auch er wurde historisch von bekannten Persönlichkeiten vertreten (v.a. Mach, 1886/2019; James, 1904a, 1904b; Russell, 1927) und wird gegenwärtig beispielsweise von Banks (2014) oder Coleman (2014) repräsentiert. Auch hier gibt es wieder eine Vielzahl an Spielarten, insbesondere wie das 
Neutrale im neutralen Monismus verstanden werden kann (Stubenberg, 2016). Im Regelfall wird unter "neutral" dasjenige verstanden, welches weder fundamental mental noch fundamental physikalisch ist. Das ist auch die Grundthese des neutralen Monismus, nämlich, dass alles neutral sei. Bei Akzeptanz des neutralen Monismus ist eine weitere metaphysische These vorhanden, die es erlaubt, sich nicht auf die Seite des Physikalisten schlagen zu müssen. Es ist die intrinsisch neutrale Natur, die schlussendlich allen mentalen und physikalischen Entitäten innewohnt.

Die am meisten diskutierteste Version eines neutralen Monismus ist der Russell'sche Monismus (siehe den Sammelband von Alter \& Nagasawa, 2015). Nach Russell liefern uns die Naturwissenschaften bloß Strukturen des Gegebenen, Zusammenhänge zwischen verschiedenen Objekten, Gesetzmäßigkeiten zwischen Ereignissen und dergleichen. Was sie uns niemals liefern, ist jedoch die intrinsische Natur des Seienden. Nichtsdestotrotz muss etwas Intrinsisches dem Physikalischen zugrunde liegen, das sicherstellt, was das Physikalische letztendlich tatsächlich ist. Hingegen beschreiben die Naturwissenschaften lediglich, wie sich das Physikalische messbar verhält. Weiters, so Russell, ist diese grundlegende, intrinsische Eigenschaft des Physikalischen auch die grundlegende, intrinsische Eigenschaft des Mentalen. Das Neutrale hat so zwei Erscheinungsweisen, wobei auf einer Seite der Medaille das Physikalische zu sehen ist und auf der anderen Seite das Mentale.

\subsubsection{Der Panprotopsychismus}

Die letzte monistische Position, der Panprotopsychismus, vertritt die Grundthese, dass alles panprotopsychistisch sei. Diese Bezeichnung entstammt Chalmers (1996), der weiterfolgend dieses Lager beschrieben hat (Chalmers, 2015), obwohl er selbst nicht als Vertreter des Panprotopsychismus verstanden werden will. Eine Argumentation, warum der Panprotopsychismus der Monismus der Wahl sein soll, findet sich beispielsweise bei Schneider (2017) und womöglich bei Coleman (2012). Chalmers versteht unter "panprotopsychistisch", dass es ein Vorläufer für etwas Mentales sei und dadurch eine spezielle Nähe zum Mentalen habe, wobei das Panprotopsychische selbst aber noch nicht mental sei. Gleichzeitig ist für Chalmers und für Schneider das Panprotopsychistische auch die intrinsische Eigenschaft von allem vermeintlich Physikalischen oder Mentalen. Der Ansatz von Coleman ist möglicherweise eine Version des Panprotopsychismus, zumindest so wie inn Chalmers interpretiert. Coleman nimmt als fundamentales Level intrinsische Entitäten an, die selbst nicht erlebende Subjekte sind wie beispielsweise typische Außenobjekte, aber die in einem weiteren Schritt prinzipiell durch ein erlebendes Subjekt wahrgenommen werden können.

Die konkrete Einordnung des Panprotopsychismus im Vergleich zu den anderen metaphysischen Positionen ist fraglich. So ordnet inn Stubenberg (2016) dem neutralen Monismus als eine Spielart des Russell'schen Monismus zu, weil panprotopsychistisch zu sein bedeutet, weder physikalisch noch mental zu sein. Chalmers scheint hingegen bewusst 
zwischen dem neutralen Monismus und dem Idealismus zu verweilen, wahrscheinlich mit einem Überhang zum Idealismus am Ende des Tages, weil er in seinen Ausführungen das Psychische im Panprotopsychismus deutlich hervorhebt. Im Vergleich dazu ordnet Schneider den Panprotopsychismus knapp außerhalb das Spektrums des Idealismus ein, weil panprotopsychistisch zu sein bedeutet, fast, aber noch nicht ganz, mental zu sein. Coleman hingegen sieht sich selbst als Panpsychist und durch die panprotopsychistische ReInterpretation von Chalmers verliert Colemans Position auch nicht die Nähe zum Panpsychismus. Ob der Panprotopsychismus als eigene monistische Position oder als eine spezifische Spielart des neutralen Monismus oder des Idealismus aufgefasst werden soll, macht für meine vorliegenden Zwecke keinen Unterschied. Es ist eine interessante Frage für sich genommen, doch ich kann sie hier nicht beantworten.

\subsection{Warum der physikalische Monismus gesichert ist}

Wenn nun der Physikalismus und seine drei konkurrierenden monistischen Positionen in allen relevanten Merkmalen vergleichbar sind, für welche Position soll man sich am Ende des Tages entscheiden? Ich bringe nachfolgend zwei Repliken zum Einwand der monistischen Gegenpositionen, hierbei ist die erste Replik das bekannte Kombinationsproblem mit einer Erweiterung auf die konkurrierenden Positionen und die zweite Replik ist ein neues Argument für den physikalischen Monismus auf Basis wahrscheinlichkeitstheoretischer Überlegungen.

\subsubsection{Das erweiterte Kombinationsproblem}

Das klassische Kombinationsproblem wurde erstmals klar in James (1890) formuliert und als solches von Seager (1995) benannt. Das Kombinationsproblem wird in der Literatur oftmals als Einwand gegen den Panpsychismus gebracht, dessen Grundthese ist, dass alles Existierende auch in einem gewissen Maße fundamental mental sei. Natürlich kann der Panpsychismus in seinen Einzelheiten noch weiter entwickelt werden, doch diese Details sind für meine Argumentation nicht wesentlich. Jedenfalls sagt das klassische Kombinationsproblem Folgendes, zumindest soweit, wie ich das Problem verstehe. Dabei ziehe ich Qualia als Beispiele heran, obwohl das Kombinationsproblem getrost auf intentionale Zustände und weitere mentale Entitäten ausgedehnt werden kann. Eben auf all diejenigen mentalen Entitäten, die unser Denken, unser Fühlen und unser gesamtes mentales Leben ausmachen:

(K klassisch) Wie können sich einzelne fundamental panpsychistische Elemente zu einem Quale kombinieren? 
Der Einwand gegen den Panpsychismus ist also der, dass der Panpsychist nicht erklären kann, wie einzelne panpsychistische Grundbausteine unserer Welt zu etwas ganzheitlichem Psychischen kombiniert werden können. Der Panpsychist muss jedoch eine Erklärung liefern, um seine Position explizit darlegen zu können.

So weit so gut zum klassischen Kombinationsproblem. Hinsichtlich der drei monistischen Rivalen möchte ich zeigen, dass auch eine abgeleitete Version des Kombinationsproblems auf sie angewandt werden kann. Ich möchte mit dem Idealismus beginnen, weil dieser ohne viele Umwege einem abgeleiteten Kombinationsproblem zum Opfer fällt:

(K idealistisch) Wie können sich einzelne fundamental mentale Elemente zu einem Quale kombinieren?

Wenn das Kombinationsproblem in einer Welt zutrifft, in der einige fundamentale Entitäten mental sind, dann trifft auch das Kombinationsproblem in einer Welt zu, in der alle fundamentalen Entitäten mental sind. Der Idealist ist mit dem Problem konfrontiert, wie aus mehreren mentalen Teilen etwas ganzheitlich Mentales wird. Er kann keine explizite Erklärung geben, wie der Mechanismus des Entstehens aussieht. Ich habe deshalb den Begriff der "Erklärung" hervorgehoben, weil diese Situation an die Erklärungslücke zwischen dem Physikalischen und Mentalen von Levine (1983) erinnert. Anstelle der Frage, wie das Mentale durch unser physikalisches Wissen erklärt werden kann, ist nun die Frage, wie das Mentale durch unser idealistisches Wissen erklärt werden kann, getreten. Vielleicht ist das Kombinationsproblem nur eine Seite der Medaille und das Problem der Erklärungslücke die andere in einem anderen verbalen Gewand. Wie auch immer die genaue Relation dieser beiden Probleme aussieht, die Lösungswahrscheinlichkeit innerhalb des Idealismus ist deutlich geringer als die innerhalb des Physikalismus. Dazu möchte ich folgende Überlegung vorbringen.

Es wäre ein physikalisches Kombinationsproblem, zu fragen, wie sich einzelne fundamentale physikalische Elemente zu einem Quale kombinieren. Oder derselbe Kern des Problems kann im Sinne der Erklärungslücke dargestellt werden, wie nämlich ein Quale durch Rekurs auf die Physik erklärt werden kann. Die Diskussionen um die dritte Prämisse des generalistischen Arguments für den Physikalismus zeigen, dass die Erklärungslücke zwischen dem Mentalen und dem Physikalischen zum Zeitpunkt der finalen Physik geschlossen werden wird. Damit ist gleichzeitig auch erreicht, dass die These des Physikalismus genau erklären kann, wie aus einzelnen fundamentalen physikalischen Elementen unser vermeintlich mentales Leben hervorgeht. Bei Betrachtung von ( $\mathrm{K}$ idealistisch) und einer möglichen idealistischen Erklärungslücke, wissen wir momentan nicht, wie solch eine vergleichbare Lösung aussehen kann. Das ist deshalb der Fall, weil keine ausgereiften idealistischen Gesetze gegeben sind, die vergleichbar sind mit den physikalischen Gesetzen. Auf Basis dieser Argumentation ist die 
Lösungswahrscheinlichkeit von (K idealistisch) geringer als parallele Probleme für den Physikalismus.

Als zweites möchte mit dem neutralen Monismus fortfahren, gegen den folgendes Kombinationsproblem vorgebracht werden kann:

(K neutral) Wie können sich einzelne fundamental neutrale Elemente zu einem Quale kombinieren?

Der neutrale Monist ist mit dem Problem konfrontiert, wie aus mehreren neutralen Teilen etwas ganzheitlich Mentales wird. Auch er kann keine explizite Erklärung geben, wie der Mechanismus des Entstehens aussieht, weshalb auch das Problem der Erklärungslücke deutlich mitschwingt. Denn wie wird die Lücke zwischen dem Mentalen und all unserem neutral-monistischen Wissen überbrückt? Bei Vergleich des neutralen Monismus mit dem Physikalismus ist wiederum die Lösungswahrscheinlichkeit innerhalb des neutralen Monismus deutlich geringer als innerhalb des Physikalismus. Der Grund ist derselbe wie weiter oben in der Diskussion des Idealismus. Wir wissen bereits sehr gut, wie sich die Erklärungslücke zwischen dem Mentalen und dem Physikalischen zum Zeitpunkt der finalen Physik schließen kann. Gleichzeitig wissen wir jedoch nicht in gleichem Maße, wie eine Lösung von ( $\mathrm{K}$ neutral) und einer möglichen neutralmonistischen Erklärungslücke aussehen kann. Der Haken für den neutralen Monisten ist, dass keine ausgereiften neutral-monistischen Gesetze gegeben sind, die vergleichbar mit den physikalischen Gesetzen sind. Auf Basis dessen ist die Lösungswahrscheinlichkeit von (K neutral) geringer als parallele Probleme für den Physikalismus.

Als drittes möchte das Kombinationsproblem für den Panprotopsychismus verdeutlichen:

(K panprotopsychistisch) Wie können sich einzelne fundamental panprotopsychistische Elemente zu einem Quale kombinieren?

Durch die starke Nähe des Panprotopsychismus zum Panpsychismus (Chalmers, 2016) bzw. zum Idealismus (Schneider, 2017) stellt sich auch hier im besonderen Maße das ähnliche Problem. Der Panprotopsychist ist mit dem Problem konfrontiert, wie aus mehreren panprotopsychistischen Teilen etwas ganzheitlich Mentales wird. Auch hier ist keine explizite Erklärung geben, wie der Mechanismus des Entstehens aussieht, weshalb auch das Problem der Erklärungslücke wieder ins Treffen geführt werden kann. Denn wie wird eine Brücke zwischen dem Mentalen und all unserem panprotopsychistischen Wissen gebaut? Bei Vergleich des Panprotopsychismus mit dem Physikalismus ist nochmals die Lösungswahrscheinlichkeit innerhalb des Panprotopsychismus deutlich geringer als innerhalb des Physikalismus. Der Grund ist ein und derselbe wie in der Diskussion des Idealismus und des neutralen Monismus. Wir können sehr gut sagen, wie sich die Erklärungslücke zwischen dem Mentalen und dem 
Physikalischen in der Ära einer vollendeten Physik schließen kann. Wir wissen hingegen nicht, wie eine Lösung von (K panprotopsychistisch) und einer möglichen panprotopsychistischmonistischen Erklärungslücke aussehen kann. Das ist vorrangig deshalb der Fall, weil wir keine ausgereiften panprotopsychistischen Gesetze haben, die vergleichbar sind mit den physikalischen Gesetzen. Deswegen ist die Lösungswahrscheinlichkeit von (K panprotopsychistisch) geringer als parallele Probleme für den Physikalismus.

\subsubsection{Bayes und das Primat des Physikalischen}

Ich möchte ein Argument vorlegen, dass sich gegen alle drei alternativen monistischen Positionen zusammen richtet. Dabei werde ich nicht einen Widerspruch in den konkurrierenden Positionen erzeugen oder die Grundannahmen ins Absurde führen. Ich kann auch keinen Beweis für ein und allemal vorlegen, dass sie falsch liegen. Was ich tun kann, ist ein Argument für das Primat des Physikalischen vorzulegen, das sich wahrscheinlichkeitstheoretisch gegen den Idealismus, den neutralen Monismus und den Panprotopsychismus durchsetzt. Den formalen Rahmen gieße ich in eine Bayes'sche Argumentationsstruktur, die sich verschiedenen A-priori-Wahrscheinlichkeiten bedient, um daraus in Verbindung mit all dem Wissen, das wir bisher in der Debatte zum Physikalismus gesammelt haben, eine A-posterioriWahrscheinlichkeit für die Wahrheit des Physikalismus bilden zu können im Vergleich zur Wahrheit der anderen drei alternativen monistischen Positionen.

Bayes war ein Mathematiker und Wahrscheinlichkeitstheoretiker im 18. Jahrhundert, der eine Formel zum Umgang mit bedingten Wahrscheinlichkeiten entwickelt hat, der später als Satz von Bayes bekannt wurde (Bayes, 1764). In der Statistik steht ein Bayes'scher Ansatz in Kontrast zu einem frequentistischen Ansatz, bei dem über bestimmte Verteilungen durch hinreichend große Beobachtungen Wahrscheinlichkeitsaussagen getroffen werden. So wird beispielsweise durch das Ziehen einer Stichprobe in der frequentistischen Statistik die Durchschnittsgröße aller Österreicher durch den Mittelwert bestimmt. Dieser Vorgang des Stichprobenziehens ist unzählige Male möglich und die Verteilungsform der Körpergröße folgt in der Bevölkerung einer Normalverteilung mit klaren, fixen Parametern wie beispielsweise der entsprechenden Standardabweichung. Hier hängt alles von den Häufigkeiten von wiederholbaren Vorgängen ab. Bei einem Bayes'schen Ansatz wird berechnet, welche Durchschnittsgröße aller Österreicher am wahrscheinlichsten ist mit der Hinzunahme von bestimmten A-priori-Annahmen. Anhand des Beispiels der Körpergröße könnte eines dieser Vorannahmen sein, dass die durchschnittliche Körpergröße so gut wie nicht über $2 \mathrm{~m}$ und auch nicht unter $1.5 \mathrm{~m}$ liegen wird. Auf Basis dieser Vorannahmen wird auf die Wahrscheinlichkeit des Eintretens eines bestimmten Falles geschätzt. Eine Verteilungsform mit klaren, fixen Parametern existiert nicht, sondern die Parameter selbst werden als Zufallsvariablen behandelt, die je nach Verteilungsform schwanken. Als fix gegeben werden die beobachteten Vorgänge angenommen. 
Hier hängt alles von unserer Überzeugtheit einer gewissen a priori zugeteilten Wahrscheinlichkeit von Vorgängen ab.

Bei metaphysischen Fragen handelt es sich nicht um Fragen, die immer einer bestimmten Verteilungsform folgen und deren Wahrscheinlichkeit, wahr zu sein, auch genau davon abhängt. Es lassen sich auch keine repräsentativen Stichproben aus der Grundgesamt ziehen. Es handelt sich bei metaphysischen Fragen vielmehr um Fragen, die von der Wertung von Argumenten und deren Prämissen abhängen. Ebenso sind metaphysische Fragen selten eindeutig und irrtumssicher zu beantworten, sondern sind in einem gewissen Maße, eben mit einer höheren oder niedrigeren Wahrscheinlichkeit, gegeben. Aus diesem Grunde bietet sich der Satz von Bayes an, um die Frage nach der Wahrheit des Physikalismus im Vergleich zu den anderen drei monistischen Alternativen besser beantworten zu können. Der Satz von Bayes ist der folgende:

$$
\text { (Bayes) } P(H \mid E)=\frac{P(H) \times P(E \mid H)}{P(H) \times P(E \mid H)+P(\neg H) \times P(E \mid \neg H)}
$$

Die einzelnen Ausdrücke werden so verstanden:

$$
\begin{aligned}
& \text { H steht für die Hypothese, in unserem Fall für den Physikalismus. } \\
& \text { E steht für die Evidenzlage. } \\
& \mathbf{P}(\mathbf{H} \mid \mathbf{E}) \text { bezeichnet die Wahrscheinlichkeit von } \mathrm{H} \text { unter der Bedingung von } \\
& \mathrm{E} \text {, also wie wahrscheinlich der Physikalismus wahr ist unter } \\
& \text { Gültigkeit der gegenwärtigen Evidenzlage. } \\
& \mathbf{P}(\mathbf{H}) \quad \text { bezeichnet die Wahrscheinlichkeit, dass } \mathrm{H} \text { wahr ist, also wie } \\
& \text { wahrscheinlich der Physikalismus wahr ist, ohne die gegenwärtige } \\
& \text { Evidenzlage einzubeziehen. }
\end{aligned}
$$

Um den Satz von Bayes auflösen zu können, müssen wir die interessierenden Parameter $P(H)$ und $\mathrm{P}(\mathrm{E} \mid \mathrm{H})$ schätzen. Der Wert von $\mathrm{P}(\neg \mathrm{H})$ ergibt sich aus der Gegenwahrscheinlichkeit $1-\mathrm{P}(\mathrm{H})$ 
und der Wert von $P(E \mid \neg H)$ ergibt sich aus der Gegenwahrscheinlichkeit $1-P(E \mid H)$. Ich will mit dem einfacheren der beiden zu schätzenden Parameter anfangen, nämlich mit $P(H)$. In diesem Fall wissen wir bereits die A-priori-Wahrscheinlichkeit, in welchem Ausmaß der Physikalismus wahr ist. Zur Auswahl stehen lediglich vier monistische Positionen: der Physikalismus, der Idealismus, der neutrale Monismus und der Panprotopsychismus. Ohne irgendwelche Evidenz miteinzubeziehen, ist jede dieser vier Möglichkeiten gleich wahrscheinlich, nämlich zu $25 \%$. Also nimmt der Wert von $\mathrm{P}(\mathrm{H})$ die Zahl 0.25 an:

$$
\mathbf{P}(\mathbf{H})=0.25
$$

Der zweite der zu schätzenden Parameter, nämlich $\mathrm{P}(\mathrm{E} \mid \mathrm{H})$ ist etwas schwieriger. Wie können wir die Wahrscheinlichkeit beziffern, dass die gegenwärtige Evidenzlage unter der Bedingung der Gültigkeit des Physikalismus wahr ist? Der Vorteil des Bayes'schen Ansatz ist, dass wir das gesamte Wissen zur Evidenzlage hier integrieren können. Hier möchte ich vor allem an die bisherigen Erkenntnisse aus den Naturwissenschaften anknüpfen, an die Evidenzlage aus Verhaltensstudien, physiologischen Studien und neurowissenschaftlichen Studien. Wir haben gesehen, dass die Erklärungslücke zwischen dem Physikalischen und dem vermeintlich Mentalen sich bereits weitestgehend geschlossen hat, eine vollendete Naturwissenschaft wird diese Lücke letztendlich komplett schließen. Dass die gegenwärtige Evidenzlage auch tatsächlich so ist, wie sie ist, falls der Physikalismus wahr ist, ist sehr hoch. Die Annahme einer intrinsischen physikalischen Natur von physikalischen Entitäten ist durchwegs eine naheliegende und intuitive Angelegenheit. Aufgrund der schwindend geringen Erklärungslücke und der naheliegenden intrinsischen physikalischen Natur des Physikalischen, ist auch die intrinsisch physikalische Natur von vermeintlich mentalen Entitäten nur einen Steinwurf entfernt.

Beachte, dass dieselbe Analyse nicht bei den anderen drei alternativen monistischen Rivalen gegeben werden kann. Denn die Annahme einer mentalen, neutralen oder panprotopsychischen intrinsischen Natur des Physikalischen liegt weiter weg und ist weniger intuitiv. Das zeigt auch die Diskussion der erweiterten Kombinationsprobleme dieser konkurrierenden Positionen. Das ist vor allem deshalb der Fall, weil die ursprüngliche Erklärungslücke zwischen dem vermeintlich Mentalen und dem Physikalischen sich im Schließen befindet und eine parallele Erklärungslücke sich weit weg vom Schließen befindet. Damit meine ich die drei Erklärungslücken unter der Gültigkeit der anderen monistischen Rivalen, nämlich zwischen Qualia und dem Mentalen/Idealistischen oder dem Neutralen oder dem Panprotopsychischen. Hier existiert noch keine Evidenzlage, wie diese Erklärungslücken geschlossen werden könnten. Was ich damit sage, ist nicht, dass die monistischen Rivalen komplett falsch liegen, sondern dass sie einfach schlechter liegen als der Physikalismus. Damit ist die Wahrscheinlichkeit der gegenwärtigen Evidenzlage unter Falschheit des Physikalismus, und damit Gültigkeit einer der drei Rivalen, äußerst gering. Aus diesem Grunde schätze ich die 
Wahrheit von $\mathrm{E}$ unter der Gültigkeit von $\mathrm{H}$ auf $99.99 \%$ ein und $\mathrm{P}(\mathrm{E} \mid \mathrm{H})$ nimmt den Wert 0.9999 an.

$$
\mathbf{P}(\mathbf{E} \mid \mathrm{H})=0.9999
$$

Nun können wir in den Satz von Bayes einsetzen:

$$
\text { (Bayes) } P(H \mid E)=\frac{0.25 \times 0.9999}{0.25 \times 0.9999+0.75 \times 0.0001}
$$

In nächsten Schritt können wir ausmultiplizieren:

$$
\text { (Bayes) } P(H \mid E)=\frac{0.249975}{0.249975+0.000075}
$$

Anschließend werden die Terme im Nenner addiert:

$$
\text { (Bayes) } P(H \mid E)=\frac{0.249975}{0.25005}
$$

Schlussendlich gelangen wir zur A-posteriori-Wahrscheinlichkeit der Wahrheit des Physikalismus unter Gültigkeit der gegenwärtigen Evidenzlage, indem wir die Division auflösen:

$$
\text { (Bayes) } P(H \mid E)=0.9997000599880024
$$

Die gerundete Wahrscheinlichkeit, dass der Physikalismus unter der gegenwärtigen Evidenzlage wahr ist, beträgt $99.97 \%$. Die Gegenwahrscheinlichkeit davon beträgt $0.03 \%$ und beziffert so die Wahrscheinlichkeit der Wahrheit aller drei alternativen monistischen Positionen zusammengenommen. Selbstverständlich hängt dieses Ergebnis von den Schätzungen der einzelnen Terme ab, doch ich habe Gründe gegeben, warum genau diejenigen Werte verwendet werden sollten, wie ich sie verwendet habe. Dieses Ergebnis ist keine letztgültige Widerlegung der drei monistischen Rivalen und vielleicht muss die Wahrscheinlichkeit, dass der Physikalismus unter der gegenwärtigen Evidenzlage wahr ist, nach unten korrigiert werden. Wie weit auch immer die Korrektur nach unten passiert, ausschlaggebend am Ende des Tages ist, dass $\mathrm{P}(\mathrm{H} \mid \mathrm{E})$ größer ist als die Wahrscheinlichkeit, dass der Idealismus, der neutrale Monismus oder der Panprotopsychismus unter der Gültigkeit der gegewärtigen Evidenzlage wahr ist ${ }^{56}$.

56 Falls nun für $\mathrm{P}(\mathrm{E} \mid \mathrm{H})$ nicht der Wert 0.9999 angenommen wird, sondern der Wert 0.5001 , so ergibt sich schlussendlich für $\mathrm{P}(\mathrm{H} \mid \mathrm{E})$ die Wahrscheinlichkeit von $25.01 \%$. Dieser Wert besagt, dass die Wahrscheinlichkeit, dass der Physikalismus unter der gegenwärtigen Evidenzlage wahr ist, $25.01 \%$ beträgt. Damit beträgt auch die 


\subsection{Zusammenfassung}

Ich habe in diesem Kapitel damit begonnen, das neue generalistische Argument für den Physikalismus vorzustellen. Die erste Prämisse sagt, falls der Physikalismus die überzeugendste Position in der Philosophie des Geistes ist, dann soll er als wahr akzeptiert werden. Die zweite Prämisse definiert drei Merkmale, wann der Physikalismus als überzeugend gilt: Wenn er im Vergleich zum Dualismus die Kriterien wissenschaftlicher Theorien besser erfüllt, wenn er die klassischen dualistischen Gedankenexperimente besser erklärt und wenn er durch wissenschaftliche Erkenntnisse besser gestützt wird. Die dritte und wichtigste Prämisse stellt fest, dass der Physikalismus genau diese drei Merkmale erfüllt. Aus diesen drei Prämissen folgt schließlich deduktiv die (vorläufige) Wahrheit des Physikalismus und der Dualismus in all seinen Ausprägungen hat das Nachsehen. Nach Verteidigung des generalistischen Arguments für den Physikalismus evaluiere ich einen möglichen Einwand, der von Vertretern alternativer monistischer Positionen in der Philosophie des Geistes vorgebracht werden könnte. Es ist der Einwand, dass mein Argument lediglich zeigt, dass der Dualismus falsch ist, jedoch nicht zeigt, dass die monistischen Rivalen falsch sind im Sinne des Idealismus, neutralen Monismus und Panprotopsychismus. Ich kontere diesem Einwand, indem ich zuerst des Arguments willen die Vergleichbarkeit zwischen dem Physikalismus und den anderen drei monistischen Rivalen in Bezug auf die Kriterien wissenschaftlicher Theorien, der Erklärung dualistischer Gedankenexperimente und der Unterstützung durch wissenschaftliche Erkenntnisse akzeptiere. Daraufhin diskutiere ich eine Familie von Kombinationsproblemen für die Konkurrenten des Physikalismus und zeige, dass die Lösungswahrscheinlichkeit der aufgebrachten Probleme geringer ist für den Idealismus, den neutralen Monismus und den Panprotopsychismus im Vergleich zu parallelen Problemen des Physikalismus. Im Anschluss darauf bringe ich ein Argument für das Primat des Physikalischen und stütze mich dabei auf probabilistische Überlegungen anhand des Satzes von Bayes. Diese wahrscheinlichkeitstheoretische Analyse zeigt, dass der Physikalismus eine sehr hohe Wahrscheinlichkeit hat, wahr zu sein, die anderen monistischen Rivalen jedoch eine sehr geringe Wahrscheinlichkeit haben, wahr zu sein. Das übergeordnete Ziel dieses Kapitels war es, die (vorläufige) Wahrheit des Physikalismus zu zeigen. Zusammengefasst habe ich durch das generalistische Argument für den Physikalismus und durch das Argument für das Primat des Physikalischen genau dieses Ziel erreicht: Der Physikalismus ist wahr, der Dualismus und andere monistische Rivalen sind falsch.

Wahrscheinlichkeit, dass eine der drei monistischen Rivalen unter der gegenwärtigen Evidenzlage wahr ist (1 - 0.2501) / 3, nämlich knapp 25.00\%, und damit ist dieser Wert unter der A-posteriori-Wahrscheinlichkeit der Wahrheit des Physikalismus. 


\section{It's OK, it's OK \\ You don't have to run and hide away}

Dead Moon (1994) 


\section{Konklusion}

Es ist morgens und wir befinden uns vor einer tiefen Schlucht, die unglaublich weit in das Erdreich nach unten führt und genau so breit ist, dass ein einfaches Überwinden durch einen weiten Sprung nicht möglich erscheint. Uns fält auf, dass diese Schlucht mit morgendlichem Nebel gefüllt ist, der etwas auf der einen und etwas auf der anderen Seite der Schlucht übertritt und sich dort an den Übertrittsstellen langsam in den umgebenen Raum auflöst. Der Rest der Schlucht ist nach wie vor aufgefüllt mit Nebel. Wenn wir auf der linken Seite der Schlucht stehen, empfinden wir Liebe, Hass, Freude und Trauer: Die gesamte Palette aller möglichen Gefühle steht uns offen, mehr oder weniger intensiv, einmal positiv gefärbt und ein anderes Mal negativ gefärbt. Wir kennen diese Gefühle aus unserer alltäglichen Erfahrung und sie zu erleben ist etwas Besonderes. Wenn wir auf der rechten Seite der Schlucht stehen, haben wir Zahlen, mathematische Formeln und Aktivitätsmuster in Gehirnen zu unserer Verfügung. Wir kennen diese Fakten aus unserer wissenschaftlichen Erfahrung und sie zu generieren ist auch etwas Besonderes. Nachdem wir auf beiden Seiten gestanden sind, verlassen wir die Schlucht und werden von einer Person aufgehalten, die sagt, dass alle Gefühle auf der linken Seite ein und dasselbe seien wie die Fakten auf der rechten Seite. Es muss sich bloß der Nebel in der Schlucht vollständig lichten und wir könnten von einer Seite auf die andere wechseln und würden so diese enge Verbindung beider ehemalig getrennten Dinge, nämlich subjektive Gefühle und naturwissenschaftliche Fakten, verstehen. Wir entgegnen dieser Person mit der Aussage, dass wir das nicht für möglich halten würden. Die Person kontert mit: "Lass es mich zeigen".

Diese Metapher der Schlucht verbildlicht die gegenwärtige Debatte in der Philosophie des Geistes zum Bewusstseinsproblem. Die fremde Person plädiert für eine physikalistische Lösung des Bewusstseinsproblems und ihr Konter, diese Lösung zeigen zu wollen, fasst die vorliegende Arbeit zusammen. Der erste wesentlichste Teil dieser Lösung umfasste eine Explizierung der These des Physikalismus als eine Typen-Identitätsthese a priori mit disjunktiver Erweiterung um den Eliminativismus. Auf dem Weg dorthin wurden neue und eigenständige Argumente entwickelt, die als essentielle Meilensteine fungierten. So wurde unter anderem gezeigt, dass Definitionsversuche des Mentalen im Rahmen der Via Negativa Hempels Dilemma neuerlich heraufbeschwören, der Physikalismus gegen das bisher unbeantwortete BlockerProblem verteidigt werden kann, das Argument der multiplen Realisierbarkeit durch ein adäquates Verständnis eines Typus entkräftet wird und der Physikalismus auch immer sowohl eine metaphysische als auch eine begriffliche These ist. Der zweite wesentliche Teil umfasste die Entwicklung eines neuen Arguments, das sogenannte generalistische Argument für den Physikalismus, welches die Wahrheit des Physikalismus deduktiv aus dessen Prämissen folgerte und sich gegen den Dualismus richtete. Ein anschließendes probabilistisches Argument 
zeigte eine höhere Wahrscheinlichkeit für die Wahrheit des Physikalismus im Vergleich zu konkurrierenden monistischen Theorien.

Um bei unserer Metapher der Schlucht zu bleiben, war am Morgen Nebel zu sehen, doch am Ende des Tages lichtet sich der Nebel immer mehr und es kommt eine solide Gesteinsschicht hervor. Das Überqueren von einer Seite auf die andere ist nun möglich, das Naheverhältnis zwischen subjektiven Gefühlen und naturwissenschaftlichen Fakten wird dadurch hinreichend erfasst. Durch ein weiteres Verflüchtigen des Nebels wird die Gesteinsverbindung zwischen den beiden Seiten immer klarer zu sehen. Es ist der Zeitpunkt einer finalen und endgültigen Physik, zu dem sich der Nebel vollständig gelichtet hat und sich das Naheverhältnis zwischen subjektiven Gefühlen und naturwissenschaftlichen Fakten auch vollständig erfassen lässt. Der Physikalismus ist eine solide, substantielle und wahre These, so wie die Gesteinsverbindung in der vermeintlichen Schlucht solide, substantiell und wahrhaftig gegeben ist. 


\section{Literatur}

Alter, T. (2007). Does representationalism undermine the knowledge argument? In T. Alter \& S. Walter (Eds.), Phenomenal concepts and phenomenal knowledge: new essays on consciousness and physicalism (S. 65-76). Oxford: Oxford University Press.

Alter, T. \& Nagasawa, Y. (2015) (Eds.). Consciousness in the physical world: perspectives on Russellian monism. Oxford: Oxford University Press.

Alter, T. \& Walter, S. (2007) (Eds.). Phenomenal concepts and phenomenal knowledge: new essays on consciousness and physicalism. Oxford: Oxford University Press.

American Psychiatric Association (2013). Diagnostic and Statistical Manual of Mental Disorders. Fifth Edition (DSM-5). Washington DC: American Psychiatric Press.

Anderson, A. K. \& Phelps, E. A. (2002). Is the human amygdala critical for the subjective experience of emotion? Evidence of intact dispositional affect in patients with amygdala lesions. Journal of Cognitive Neuroscience, 14, 709-720.

Aranyosi, I. (2011). A new argument for mind-brain identity. British Journal of Philosophy of Science, 62, 489-517.

Armstrong, D. (1968). A materialist theory of the mind. London: Routledge and Kegan Paul.

Baars, B. J. (2019). On consciousness: science and subjectivity. New York: Nautilus Press.

Baker, A. (2011). Simplicity. In E. N. Zalta (Ed.), The Stanford Encyclopedia of Philosophy. Online: https://plato.stanford.edu/entries/mind-identity/ [05.07.2021].

Banks, E. C. (2014). The realistic empiricism of Mach, James, and Russell. Neutral monism reconsidered. Cambridge: Cambridge University Press.

Bar-Haim, Y., Lamy, D., Pergamin, L., Bakermans-Kranenburg, M. J. \& van ljzendoorn, M. H. (2007). Threat-related attentional bias in anxious and nonanxious individuals: a metaanalytic study. Psychological Bulletin, 133, 1-24.

Barwich, A. (2019). The value of failure in science: the story of grandmother cells in neuroscience. Frontiers in Neuroscience, 13: 1121.

Online: https://www.frontiersin.org/articles/10.3389/fnins.2019.01121/full [05.07.2021].

Barrett, L. F. (2017). How emotions are made: the secret life of the brain. Boston: Houghton Mifflin Harcourt.

Barrett, L. F., Mesquita, B., Ochsner, K. N. \& Gross, J. J. (2007). The experience of emotion. Annual Review of Psychology, 58, 373-403.

Bayes, T. (1764). An essay toward solving a problem in the doctrine of chances. Philosophical Transactions of the Royal Society of London, 53, 370-418.

Baysan, U. (2018). Epiphenomenal properties. Australasian Journal of Philosophy, 96, 419-431.

Beauregard, M. (2007). Mind does really matter: evidence from neuroimaging studies of emotion self-regulation, psychotherapy, and placebo effects. Progress in Neurobiology, 81, 218236. 
Bechtel, W. (2012). Identity, reduction, and conserved mechanism: perspectives from circadian rhythm research. In S. Gozzano \& C. Hill (Eds.), The mental, the physical: new perspectives on type identity (S. 37-48). Pittsburgh: University of Pittsburgh Press.

Bechtel, W. \& Mundale, J. (1999). Multiple realizability revisited: linking cognitive and neural states. Philosophy of Science, 66, 175-207.

Berkeley, G. (1734). A treatise concerning the principles of human knowledge. Dublin: Aaron Rhames.

Bickle, J. (2006). Reducing mind to molecular pathways: explicating the reductionism implicit in current cellular and molecular neuroscience. Synthese, 151, 411-434.

Bird, A. (2007). Inference to the only explanation. Philosophy and Phenomenological Research, 74, 424-432.

Bishop, R. C. (2006). The hidden premise in the causal argument for physicalism. Analysis, 66, 44-52.

Block, N. (1978). Troubles with functionalism. Reprinted (revised version) in N. Block (Ed.), Readings in the philosophy of psychology, Vol. 1, 1980, (S. 268-305). Cambridge: Harvard University Press.

Block, N. (1980). Introduction: What is functionalism? In N. Block (Ed.), Readings in the philosophy of psychology, Vol. 1, (S. 171-184). Cambridge: Harvard University Press.

Block, N. \& Foder, J. (1972). What psychological states are not. The Philosophical Review, 81, 159-181.

Block, N. \& Stalnaker, R. (1999). Conceptual analysis, dualism, and the explanatory gap. Philosophical Review, 108, 1-46.

Blumson, B. \& Tang, W. H. (2015). A note on the definition of physicalism. Thought, 4, 10-18.

Boghossian, P. (1990). The status of content. Philosophical Review, 99, 157-184.

Bradley, D. (2018). Philosophers should prefer simpler theories. Philosophical Studies, 175, 3049-3067.

Brenner, A. (2017). Simplicity as a criterion of theory choice in metaphysics. Philosophical Studies, 174, 2687-2707.

Brentano, F. (1874/2008). Psychologie vom empirischen Standpunkt. Von der Klassifikation psychischer Phänomene (Franz Brentano - Sämtliche veröffentlichte Schriften, Band I). Frankfurt am Main: Ontos Verlag.

Bunge, M. (1963). The myth of simplicity. New Jersey: Prentice-Hall, Englewood Cliffs.

Buxton, R. B. (2013). The physics of functional magenetic resonance imaging (fMRI). Reports on Progress in Physics.

Online: https://iopscience.iop.org/article/10.1088/0034-4885/76/9/096601 [05.07.2021].

Cao, J., Liu, Q., Li, Y., Yang, J., Gu, R., Liang,J., Qi, Y., Wu, H. \& Liu, X. (2017). Cognitive behavioural therapy attenuates the enhanced early facial stimuli processing in social 
anxiety disorders: an ERP investigation. Behavioral and Brain Functions, 13:12.

Online: https://europepmc.org/article/med/28754179\#free-full-text [05.07.2021].

Carnap, R. (1931). Die physikalische Sprache als Universalsprache der Wissenschaft.

Erkenntnis, 2, 432-465.

Carnap, R. (1932). Psychologie in physikalischer Sprache. Erkenntnis, 3, 107-142.

Carruthers, P. (2000). Phenomenal consciousness: a naturalistic theory. Cambridge, MA: Cambridge University Press.

Chalmers, D. J. (1996). The conscious mind: in search of a fundamental theory. New York:

Oxford University Press.

Chalmers, D. J. (2000). What is a neural correlate of consciousness? In T. Metzinger (Ed.), Neural correlates of consciousness: empirical and conceptual questions (S. 17-40). Cambridge: MIT Press.

Chalmers, D. J. (2003). The content and epistemology of phenomenal belief. In Q. Smith \& A. Jokic. (Eds.), Consciousness: new philosophical perspectives (S. 220-272). Oxford: Oxford University Press.

Chalmers, D. J. (2004). The representational character of experience. In B. Leiter (Ed.), The future of philosophy (S. 153-181). New York: Oxford University Press.

Chalmers, D. J. (2007). Phenomenal concept and the explanatory gap. In T. Alter \& S. Walter (Eds.), Phenomenal concepts and phenomenal knowledge: new essays on consciousness and physicalism (S. 167-194). Oxford: Oxford University Press.

Chalmers, D. J. (2010). The two-dimensional argument against materialism. In D. J. Chalmers, The character of consciousness (S. 141-191). Oxford: Oxford University Press.

Chalmers, D. J. (2011). Revisability and conceptual change in "Two dogmas of empiricism". The Journal of Philosophy, 108, 387-415.

Chalmers, D. J. (2012). Constructing the world. Oxford: Oxford University Press.

Chalmers, D. J. (2015). Panpsychism and panprotopsychism. In T. Alter \& Y. Nagasawa (Eds.), Consciousness in the physical world: perspectives on Russellian monism (S. 246-276). Oxford: Oxford University Press.

Chalmers, D. J. (2016). The combination problem for panpsychism. In L. Jaskolla \& G. Bruntrup (Eds.), Panpsychism (S. 179-214). Oxford: Oxford University Press.

Chalmers, D. J. (2018). The meta-problem of consciousness. Journal of Consciousness Studies, $25,6-61$.

Chalmers, D. J. (2019). Idealism and the mind-body problem. In W. Seager (Ed.), The Routledge Companion to panpsychism (S. 353-373). Oxford: Oxford University Press.

Chalmers, D. J. \& Jackson, F. (2001). Conceptual analysis and reductive explanation. The Philosophical Review, 110, 315-360.

Chalmers, J. A., Heathers, J. A. J., Abbott, M. J., Kemp, A. H. \& Quintana, D. S. (2016). Worry is associated with robust reductions in heart rate variability: a transdiagnostic study of 
anxiety psychopathology. BioMed Central Psychology, 4:32.

Online: https://bmcpsychology.biomedcentral.com/articles/10.1186/s40359-016-0138-z [05.07.2021].

Chisholm, R. M. (1957). Perceiving. Ithaca: Cornell.

Chomsky, N. (2006). Language and mind. Third edition. Cambridge: Cambridge University Press.

Churchland, P. M. (1981). Eliminative materialism and the propositional attitudes. Journal of Philosophy, 78, 67-90.

Churchland, P. M. (2007). The evolving fortunes of eliminative materialism. In B. P. McLaughlin \& J. C. Malden (Eds.), Contemporary debates in philosophy of mind (S. 160-181). Cambridge: Blackwell.

Churchland, P. S. (1995). Die Neurobiologie des Bewußtseins. Was können wir von ihr lernen? In T. Metzinger (Ed.), Bewußtsein - Beiträge aus der Gegenwartsphilosophie (S. 463-490). Paderborn: mentis.

Churchland, P. S. (1989). Neurophilosophy: toward a unified science of the mind-brain. Cambridge, MA: MIT Press.

Churchland, P. S. (2005). A neurophilosophical slant on consciousness research. Progress in Brain Research, 149, 285-293.

Churchland, P. S. (2008). The impact of neuroscience on philosophy. Neuron, 60, 409-411.

Coleman, S. (2012). Mental chemistry: combination for panpsychists. Dialectica, 66, 137-166.

Coleman, S. (2014). The real combination problem: panpsychism, micro-subjects, and emergence. Erkenntnis, 79, 19-44.

Coleman, S. (2019) (Ed.). The knowledge argument. Cambridge: Cambridge University Press. Corns, J. (2016). Pain eliminativism: scientific and traditional. Synthese, 193, 2949-2971.

Crane, T. (1998). Intentionality as the mark of the mental. In A. O'Hear (Ed.), Contemporary issues in the philosophy of mind (S. 229-251). Cambridge: Cambridge University Press.

Crane, T. \& Mellor, D. H. (1990). There is no question of physicalism. Mind, 99, 185-206.

Crick, F. \& Koch, C. (1990). Towards a neurobiological theory of consciousness. Seminars in the Neurosciences, 2, 263-275.

Crook, S., \& Gillett, C. (2001). Why physics alone cannot define the 'physical': materialism, metaphysics, and the formulation of physicalism. Canadian Journal of Philosophy, 31, 333-360.

Daly, C. (2013). Psychology and indispensability. The Monist, 96, 561-581.

Damasio, A. R. (1994). Descartes's error: emotion, reason, and the human brain. New York: Avon Books Inc.

Damasio, A. \& Carvalho, G. B. (2013). The nature of feelings: evolutionary and neurobiological origins. Nature Reviews Neuroscience, 14, 143-152. 
Dasgupta, S. (2014). The possibility of physicalism. Journal of Philosophy, 111, 557-592.

Davidson, D. (1970). Mental events. Reprinted in D. J. Chalmers (Ed.), Philosophy of mind: classical and contemporary readings, 2002, (S. 116-125). Oxford: Oxford University Press.

Dennett, D. C. (1988). Quining qualia. Reprinted in W. Lycan (Ed.), Mind and cognition, 1990, (S. 519-548). Oxford: Blackwells.

Dennett, D. C. (2016). Illusionism as the obvious default theory of consciousness. Journal of Consciousness Studies, 23, 65-72.

Descartes, R. (1641). Meditationes de prima philosophia. In G. Schmidt (Ed.), Meditationen über die Erste Philosophie, 1986. Stuttgart: Reclam.

Descartes, R. (1649). Die Passionen der Seele. In C. Wohlers (Ed.), Die Passion der Seele, 2014. Stuttgart: Meiner.

D'oro, G., Giladi, P. \& Papazoglou, A. (2019). Non-reductivism and the metaphilosophy of mind. Inquiry, 62, 477-503.

Dove, G. (2018). Redefining physicalism. Topoi, 37, 513-522.

Dowell, J. L. (2006). The physical: empirical, not metaphysical. Philosophical Studies, 131, 2560.

Dretske, F. (1995). Naturalizing the mind. Cambridge: MIT Press.

Duhem, P. (1998). Ziel und Struktur der physikalischen Theorien. Hamburg: Felix Meiner Verlag.

Eccles, J. C. (1989). Evolution of the brain: creation of the self. New York: Routledge.

Ekman, P. (1999). Basic emotions. In T. Dalgleish \& T. Power (Eds.), The handbook of cognition and emotion (S. 45-60). New York: John Wiley \& Sons.

Elpidorou, A. \& Dove, G. (2018). Consciousness and physicalism. A defense of a research program. New York: Routledge.

Etkin, A. \& Wager, T D, (2007). Functional neuroimaging of anxiety: a meta-analysis of emotional precessing in PTSD, social anxiety disorder, and specific phobia. American Journal of Psychiatry, 164, 1476-1488.

Farrell, B. A. (1950). Experience. Mind, 59, 170-198.

Feigl, H. (1958/1967). The "mental" and the "physical", in H. Feigl, M. Scriven \& G. Maswell (Eds.), 1958, Concepts, theories and the mind-body problem. Minnesota studies in the philosophy of science Vol. 2 (S. 370-497). Minneapolis: University of Minnesota Press. Reprinted in Feigl, H., 1967, The "mental" and the "physical". The essay and a postscript. Minneapolis: University of Minnesota Press.

Feinstein, J. S., Buzza, C., Hurlemann, R., Follmer, R. L., Dahdeleh, N. S., Coryell, W. H., Welsh, M. J., Tranel, D. \& Wemmie, J. A. (2013). Fear and panic in humans with bilateral amygdala damage. Nature Neuroscience, 16, 270-273.

Feyerabend, P. (1963). Comment: "Mental events and the brain". Journal of Philosophy, 40, 295-296. 
Fine, K. (2001). The question of realism. Philosophers' Imprint, 1, 1-30.

Fiorese, R. (2016). Stoljar's dilemma and three conception of the physical: a defence of the via negativa. Erkenntnis, 81, 201-229.

Fodor, J. A. (1974). Special sciences, or the disunity of science as a working hypthesis. Synthese, 28, 97-115.

Foster, J. (1991). The immaterial self. London: Routledge.

Foster, J. (2008). A world for us: the case for phenomenalistic idealism. Oxford: Oxford University Press.

Francescotti, F. (2014). The problem of extras and the contingency of physicalism. Philosophical Exploration, 17, 241-254.

Frankish, K. (2016). Illusionism as a theory of consciousness. Journal of Consciousness Studies, 23, 11-39.

Frege, G. W. (1892). Über Sinn und Bedeutung. Zeitschrift für Philosophie und Philosophische Kritik, 100, 25-50.

Gadenne, V. (2006). In defence of qualia-epiphenomenalism. Journal of Consciousness Studies, 13, $101-114$.

Gillett, C. \& Witmer, G. (2001). A 'physical' need: physicalism and the via negativa. Analysis, 61, 302-309.

Gillett, C. \& Loewer, B. (2001) (Eds.). Physicalism and its discontents. Cambridge: Cambridge University Press.

Goff, P. (2017). Consciousness and fundamental reality. Oxford: Oxford University Press.

Goldschmidt, T. \& Pearce, K. L. (2017) (Eds.). Idealism: new essays in metaphysics. Oxford: Oxford University Press.

Gole, M. (2008a). Reduction revisited: the ontological level, the conceptual level, and the tenets of physicalism. In A. Hieke \& H. Leitgeb (Eds.), Reduction and elimination in philosophy and the sciences (S. 125-127). Frankfurt am Main: Ontos Press.

Gole, M. (2008b). Das Argument des unvollständigen Wissens: Physikalismus versus Dualismus. Unveröffentlichte Diplomarbeit, Karl-Franzens-Universität Graz.

Gole, M., Köchel, A., Schäfer, A. \& Schienle, A. (2012). Threat engagement, disengagement, and sensitivity bias in worry-prone individuals as measured by an emotional go/no-go task. Journal of Behavior Therapy and Experimental Psychiatry, 43, 532-539.

Gomez, P., Zimmermann, P., Guttormsen-Schär, S. \& Danuser, B. (2005). Respiratory response associated with affective processing of film stimuli. Biological Psychology, 68, 223-235.

Gottlieb, S. \& Lomrozo, T. (2018). Can science explain the human mind? Intuitive judgements about the limits of science. Psychological Science, 29, 121-130.

Graeff, F. G. \& Junior, H. Z. (2010). The hypothalamic-pituitary-andrenal axis in anxiety and panic. Psychology \& Neuroscience, 3, 3-8. 
Grindeland, J. (2015). Blockers: a reply to Hawthorne. Epistemology \& Philosophy of Science, $44,112-124$.

Harman, G. (1990). The intrinsic quality of experience. Philosophical Perspectives, 4, 31-52.

Hart, W. D. (1988). The engines of the soul. Cambridge: Cambridge University Press.

Hawthorne, J. P. (2002). Blocking definitions of materialism. Philosophical Studies, 110, 103113.

Hellman, G. (1985). Determination and logical truth. Journal of Philosophy, 82, 607-616.

Hempel, C. (1935). Analyse logique de la psychologie. Revue de Synthèse, 9-10, 27-42.

Reprinted in N. Block (Ed.), 1980, Readings in the Philosophy of Psychology, Volume 1

(S. 14-23). Cambridge: Harvard University Press.

Hempel, C. (1969). Reduction: ontological and linguistic facets. In S. Morgenbesser, P. Suppes

\& M. White (Eds.), Philosophy, science, and method: essays in honor of Ernest Nagel

(S. 179-99). New York: St. Martin's Press.

Hempel, C. (1980). Comments on Goodman's ways of worldmaking. Synthese, 45, 193-199.

Hill, C. (1991). Sensations: a defense of type physicalism. Cambridge: Cambridge University Press.

Horgan, T. (1993). From supervenience to superdupervenience: meeting the demands of a material world. Mind, 102, 555-586.

Horgan, T. (2006). Materialism: matters of definition, defense, and deconstruction. Philosophical Studies, 131, 157-157.

Horgan, T. \& Tienson, J. (2001). Deconstructing new wave materialism. In C. Gillett \& B. Loewer (Eds.), Physicalism and its discontents (S. 307-318). Cambridge: Cambridge University Press.

Huemer, M. (2009). When is parsimony a virtue? The Philosophical Quarterly, 59, 216-236.

Huxley, T. H. (1874). On the hypothesis that animals are automata, and its history. The Fortnightly Review, 16, 555-580.

Jackson, F. (1982). Epiphenomenal qualia. Philosophical Quarterly, 32, 127-136.

Jackson, F. (1986). What Mary didn't know. Journal of Philosophy, 83, 291-295.

Jackson, F. (1998a). From metaphysics to ethics: a defense of conceptual analysis. Oxford: Clarendon.

Jackson, F. (1998b). Postscript on qualia. In F. Jackson, Mind, method and conditionals: selected essays (S. 76-79). London: Routledge.

Jackson, F. (2007). A priori physicalism. In B. P. McLaughlin \& J. C. Malden (Eds.), Contemporary debates in philosophy of mind (S. 185-199). Cambridge: Blackwell.

Jackson, F. (2012). In defence of the identity theory mark I. In C. Hill \& S. Gazzano (Eds.), New perspectives on type identity: the mental and the physical (S. 150-166). Cambridge: Cambridge University Press.

James, W. (1890). The principles of psychology. New York: Dover Publications. 
James, W. (1904a). A world of pure experience. Journal of Philosophy, Psychology and Scientific Methods, 1, 533-543, 561-570.

James, W. (1904b). Does 'consiousness' exist? Journal of Philosophy, Psychology and Scientific Methods, 1, 477-491.

Judisch, N. (2008). Why 'non-mental' won't work: on Hempel's dilemma and the characterization of the 'physical'. Philosophical Studies, 140, 299-318.

Kant, I. (1787/1929). Critique of pure reason. Übersetzt von N. Kemp Smith. New York: St. Martin's Press.

Kassam, K. S., Markey, A. R., Cherkassky, V. L., Loewenstein, G. \& Just, M. A. (2013). Identifying emotions on the basis of neural activation. PLoS One, 8.

Online: https://journals.plos.org/plosone/article?id=10.1371/journal.pone.0066032 [05.07.2021].

Kastrup, B. (2017). An ontological solution to the mind-body problem. Philosophies, 2:10. Online: https://www.mdpi.com/2409-9287/2/2/10 [05.07.2021].

Kendler, K. S. (2005). Toward a philosophical structure for psychiatry. American Journal of Psychiatry, 162, 433-440.

Kim, J. (1984). Concepts of supervenience. Philosophy and Phenomenological Research, 45, 153-176.

Kim, J. (1987). "Strong" and "global" supervenience revisited. Philosophy and Phenomenological Research, 48, 315-326.

Kim, J. (1989). The myth of nonreductive materialism. Proceedings and Addresses of the American Philosophical Association, 63, 31-47.

Kim, J. (1993). Supervenience and mind: selected philosophical essays. Cambridge: Cambridge University Press.

Kim, J. (2005). Physicalism, or something near enough. Princeton: Princeton University Press.

Kim, J. (2012). The very idea of token physicalism. In C. Hill \& S. Gozzano (Eds.), New perspectives on type identity: the mental and the physical (S. 167-185). Cambridge: Cambridge University Press.

Kim, M. J., Gee, D. G., Loucks, R. A., Davis, F. C. \& Whalen, P. J. (2011). Anxiety dissociates dorsal and ventral medial prefrontal cortex functional connectivity with the amygdala at rest. Cerebral Cortex, 21, 1667-1673.

Kirk, R. (1974a). Sentience and behaviour. Mind, 83, 43-60.

Kirk, R. (1974a). Zombies v. materialists. Proceedings of the Aristotelian Society, 48 (Supplementary), 135-152.

Knight, L. K. \& Depue, B. E. (2019). New frontiers in anxiety research: the translational potential of the bed nucleus of the stria terminalis. Frontiers in Psychiatry, 10: 510.

Online: https://www.frontiersin.org/articles/10.3389/fpsyt.2019.00510/full [05.07.2021]. 
Knotts, J. D., Odegaard, B. \& Lau, H. (2018). Neuroscience: the key to consciousness may not be under streetlight. Current Biology, 28, R749-R752.

Koch, C., Massimini, M., Boly, M. \& Tononi, G. (2016). Neural correlates of consciousness: progress and problems. Nature Reviews Neuroscience, 17, 307-321.

Kripke, S. (1980). Naming and necessity. Cambridge: Harvard University Press.

Kuhn, T. (1967). Die Struktur wissenschaftlicher Revolutionen. Frankfurt: Suhrkamp Verlag.

Lakatos, I. (1982). Die Methodologie der wissenschaftlichen Forschungsprogramme. Berlin: Springer Verlag.

Langsam, H. (2020). Why intentionalism cannot explain phenomenal character. Erkenntnis, 85, 375-389.

LeDoux, J. E. \& Brown, R. (2017). A higher-order theory of emotional consciousness. PNAS, E2016-E2025.

León-Domínguez, U. \& León-Carrión, J. (2019). Prefrontal neural dynamics in consciousness. Neuropsychologia, 131, 25-41.

Leuenberger, S. (2008). Ceteris absentibus physicalism. In D. Zimmerman (Ed.), Oxford Studies in Metaphysics (S. 145-170). Oxford: Oxford University Press.

Leutgeb, V., Schäfer, A. \& Schienle, A. (2006). An event-related potential study on exposure therapy for patients suffering from spider phobia. Biological Psychology, 82, 293-300.

Levin, J. (2018). Functionalism. In E. N. Zalta (Ed.), The Stanford Encyclopedia of Philosophy. Online: https://plato.stanford.edu/entries/functionalism/ [26.10.2020].

Levine, J. (1983). Materialism and qualia: the explanatory gap. Pacific Philosophical Quarterly, 64, 354-361.

Levine, J. (1993). On leaving out what it's like. In M. Davies \& G. Humphreys (Eds.), Consciousness: psychological and philosophical essays (S. 121-136). Oxford: Blackwell.

Levine, J. (2003). Experience and representation. In Q. Smith \& A. Jokic. (Eds.), Consciousness: new philosophical perspectives (S. 57-76). Oxford: Oxford University Press.

Levine, J. (2018). Quality and content: essays on consciousness, representation, and modality. Oxford: Oxford University Press.

Lewis, C. I. (1929). Mind and the world order. New York: Charles Sribner's Sons.

Lewis, D. (1966). An argument for the identity theory. Journal of Philosophy, 63, 17-25.

Lewis, D. (1983). New work for a theory of universals. Australasian Journal of Philosophy, 61, 343-377.

Lewis, D. (1994). Reduction of mind. In. S. Guttenplan (Ed.), A companion to the philosophy of mind (S. 412-431). Oxford: Blackwell.

Libet, B. (2006). Reflections on the interaction of the mind and brain. Progress in Neurobiology, 78, 322-326.

Loar, B. (1997). Phenomenal states. Revised version in N. Block, O. Flanagan \& G.

Güzeldere (Eds.), The nature of consciousness (S. 597-616). Cambridge: MIT Press. 
Locke, J. (1689). Essay concerning human understanding. London: Eliz. Holt.

Logothetis, N. K. (2002). The neural basis of the blood-oxygen-level-dependent functional magnetic resonance imaging signal. Philosophical Transactions of the Royal Society of London B, 357, 1003-1037.

Lycan, W. G. (2009). Giving dualism its due. Australasian Journal of Philosophy, 87, 551-563.

Lycan, W. G. (2013). Is property dualism better off than substance dualism? Philosophical Studies, 164, 533-542.

Lycan, W. G. (2019). Representational theories of consciousness. In E. N. Zalta (Ed.), The Stanford Encyclopedia of Philosophy. Online:

https://plato.stanford.edu/entries/consciousness-representational/ [26.10.2020].

Mach, E. (1886/2019). Die Analyse der Empfindungen und das Verhältnis des Physischen zum Psychischen. Norderstedt: Vero Verlag.

Machery, E. (2009). Doing without concepts. Oxford: Oxford University Press.

MacLeod, C., Grafton, B. \& Notebaert, L. (2019). Anxiety-linked attentional bias: is it reliable? Annual Review of Clinical Psychology, 15, 529-554.

MacPherson, F. (2000). Representational theories of phenomenal character. Ph.D. Thesis, University of Stirling.

Malebranche, N. (1997). Dialogues on metaphysics and on religion. In N. Jolley \& D. Scott (Eds.). Cambridge: Cambridge University Press.

Mazzi,C., Mazzeo, G. \& Savazzi, S. (2017). Markers of TMS-evoked visual conscious experience in a patient with altitudinal hemianopia. Consciousness and Cognition, 54, 143-154.

McLaughlin, B. P. (2001). In defense of new wave materialism: a response to Horgan and Tienson. In C. Gillett \& B. Loewer (Eds.), Physicalism and its discontents (S. 319-330). Cambridge: Cambridge University Press.

McLaughlin, B. P. (2007). On the limits of a priori physicalism. In B. P. McLaughlin \& J. C. Malden (Eds.), Contemporary debates in philosophy of mind (S. 200-223). Cambridge: Blackwell.

Melnyk, A. (1997). How to keep the "physical" in physicalism. Journal of Philosophy, 94, 622637.

Melnyk, A. (2003). A physicalist manifesto: thoroughly modern materialism. Cambridge: Cambridge University Press.

Melnyk, A. (2016). Grounding and the formulation of physicalism. In K. Aizawa \& C. Gillett (Eds.), Scientific composition and metaphysical ground. New directions in the philosophy of science (S. 249-269). London: Palgrave Macmillan.

Mogi, K. (2013). Cognitive factors correlating with the metacognition of the phenomenal properties of experience. Scientific Reports, 3: 3354. Online:

https://www.nature.com/articles/srep03354 [05.07.2021]. 
Montero, B. (1999). The body problem. Nous, 33, 183-200.

Montero, B. (2001). Post-Physicalism. Journal of Consciousness Studies, 8, 61-80.

Montero, B. (2006). Physicalism in an infinitely decomposable world. Erkenntnis, 64, 177-191.

Montero, B. (2009). What is the physical? In B. Mclaughlin, A. Beckermann \& S. Walter (Eds.), The Oxford Handbook of philosophy of mind (S. 173-188). Oxford: Oxford University Press.

Montero, B. (2013). Must physicalism imply the supervenience of the mental on the physical? The Journal of Philosophy, 110, 93-110.

Montero, B. \& Papineau, D. (2005). A defence of the via negativa argument for physicalism. Analysis, 65, 233-237.

Mulligan, K. (2004). Brentano on the mind. In D. Jacquette (Ed.), The Cambridge Companion to Brentano (S. 66-97). Cambridge: Cambridge University Press.

Nagasawa, Y. (2010). The knowledge argument and epiphenomenalism. Erkenntnis, 72, 37-56. Nagel, T. (1974). What is it like to be a bat? Philosophical Review, 83, 435-450.

Neurath, O. (1931). Soziologie im Physikalismus. Erkenntnis, 2, 393-431.

Ney, A. (2008). Physicalism as an attitude. Philosophical Studies, 138, 1-15.

Nida-Rümelin, M. (2007). Grasping phenomenal properties. In T. Alter \& S. Walter (Eds.),

Phenomenal concepts and phenomenal knowledge: new essays on consciousness and physicalism (S. 307-338). Oxford: Oxford University Press.

Nida-Rümelin, M. (2019). Qualia: the knowledge argument. In E. N. Zalta (Ed.), The Stanford Encyclopedia of Philosophy. Online: https://plato.stanford.edu/entries/qualia-knowledge/ [05.07.2021].

Olejniczak, P. (2006). Neurophysiological basis of EEG. Journal of Clinical Neurophysiology, 23, 186-189.

Panksepp, J., Lane, R. D., Solms, M. \& Smith, R. (2017). Reconciling cognitive and affective neuroscience perspectives on the brain basis of emotional experience. Neuroscience and Biobehavioral Reviews, 76, 187-215.

Papineau, D. (2002). Thinking about consciousness. New York: Oxford University Press.

Papineau, D. (2007). Phenomenal and perceptual concepts. In T. Alter \& S. Walter (Eds.), Phenomenal concepts and phenomenal knowledge: new essays on consciousness and physicalism (S. 111-144). Oxford: Oxford University Press.

Pereboom, D. \& Kornblith, H. (1991). The metaphysics of irreducibility. Philosophical Studies, 63, 125-145.

Perry, J. (1979). The problem of the essential indexical. Nous, 13, 3-21.

Perry, J. (2001). Knowledge, possibility, and consciousness. Cambridge, MA: MIT Press.

Place, U. T. (1956). Is consciousness a brain process? British Journal of Psychology, 47, 4450. 
Pluess, M., Conrad, A. \& Wilhelm, F. H. (2009). Muscle tension in generalized anxiety disorder: a critical review the literature. Journal of Anxiety Disorders, 23, 1-11.

Popper, K. (1934). Logik der Forschung. Wien: Springer Verlag.

Popper, K. (1994). Knowledge and the body-mind problem. In defence of interaction. London: Routledge.

Poslajko, K. (2019). Eliminativism: the problem of representation and Carnapian metametaphysics. Acta Analytica, 34, 181-195.

Prelevic, D. (2017). Hempel's dilemma and research programmes: why adding stances is not a boon. Organon F, 24, 487-510.

Putnam, H. (1967). Psychological predicates. In W. H. Capitan \& D. D. Merrill (Eds.), Art, mind, and religion (S. 37-48). Pittsburgh: University of Pittsburgh Press.

Putnam, H. (1975). The meaning of "meaning". Language, mind, and knowledge. Minnesota studies in the philosophy of science, 7, 131-193.

Quine, W. V. O. (1948). On what there is. Review of Metaphysics, 2, 21-38.

Quine, W. V. O. (1951). Two dogmas of empiricism. The Philosophical Review, 60, 20-43.

Quine, W. V. O. (1960). Word and object. Cambridge: MIT Press.

Quine, W. V. O. \& Ullian, J. S. (1970). The web of belief. New York: Random House.

Rabin, G. O. (2020). Fundamentality physicalism. Inquiry, DOI: 10.1080/0020174X.2019.1688177.

Rorty, R. (1965). Mind-body identity, privacy, and categories. Review of Metaphysics, 19, 24-54.

Rorty, R. (1970). In defense of eliminative materialism. Review of Metaphysics, 24, 112-121.

Rosebrock, L. E., Hoxha, D., Norris, C., Cacioppo, J. T. \& Gollan, J. K. (2016). Skin conductance and subjective arousal in anxiety, depression, and comorbidity. Journal of Psychophysiology, 31, 145-157.

Rosen, G. (2010). Metaphysical dependence: grounding and reduction. In B. Hale \& A. Hoffmann (Eds.), Modality: metaphysics, logic, and epistemology (S. 109-136). Oxford: Oxford University Press.

Russell, B. (1905). On denoting. Mind, 14, 479-493.

Russell, B. (1927). The analysis of matter. London: George Allen \& Unwin.

Ryle, G. (1949). The concept of mind. London: Hutchinson.

Sacchetti, A., Mattei, G, Bursi, S., Padula, M. S., Rioli, G. \& Ferrari, S. (2017). Association of blood pressure with anxiety and depression in a sample of primary care patients. European Psychiatry, 41, Supplement, S496.

Schaffer, J. (2009). On what grounds what. In D. Chalmers, D. Manley \& R. Wasserman (Eds.), Metametaphysics: new essays on the foundations of ontology (S. 347-383). Oxford: Oxford University Press. 
Schneider, S. (2017). Idealism, or something near enough. In T. Goldschmidt \& K. L. Pearce (Eds.), Idealism: new essays in metaphysics (S. 275-290). Oxford: Oxford University Press.

Searle, J. R. (1983). Intentionality. Cambridge: Cambridge University Press.

Searle, J. R. (2002). Why I am not a property dualist. Journal of Consciousness Studies, 9, 5764.

Seager, W. (1995). Consciousness, information, and panpsychism. Journal of Consciousness Studies, 2, 272-88.

Seager, W. (2019) (Ed.). The Routledge Companion to panpsychism. Oxford: Oxford University Press.

Sejnowsky, T. J., Churchland, P. S. \& Movshon, J. A. (2014). Putting big data to good use in neuroscience. Nature Neuroscience, 17, 1440-1441.

Shoemaker, S. (2007). Physical realization. Oxford: Oxford University Press.

Smart, J. J. C. (1959). Sensation and brain processes. Reprinted in P. K. Moser \& J. D. Trout (Eds.), Contemporary materialism: a reader, 1995, (S. 93-106). London: Routledge.

Smart, J. J. C. (2007). The mind/brain identity theory. In E. N. Zalta (Ed.), The Stanford Encyclopedia of Philosophy. Online: https://plato.stanford.edu/entries/mind-identity/ [05.07.2021].

Sober, E. (1998). Simplicity (in scientific theories). In E. Craig (Ed.), Routledge Encyclopedia of Philosophy (S. 780-783). London: Routledge.

Sober, E. (2001). What is the problem of simplicity? In A. Zellner, H. Keuzenkamp \& M. McAleer (Eds.), Simplicity, inference, and modeling: keeping it sophisticatedly simple (S. 13-31). Cambridge: Cambridge University Press.

Spurrett, D. \& Papineau, D. (1999). A note on the completeness of 'physics'. Analysis, 59, 25-29.

Stich, S. (1996). Deconstructing the mind. New York: Oxford University Press.

Stoljar, D. (2001). Two conceptions of the physical. Philosophy and Phenomenological Research, 62, 253-281.

Stoljar, D. (2005). Physicalism and phenomenal concepts. Mind and Language, 20, 469494.

Stoljar, D. (2006). Ignorance and imagination. Oxford: Oxford University Press.

Stoljar, D. (2010). Physicalism. London: Routledge.

Stoljar, D. (2015). Physicalism. In E. N. Zalta (Ed.), The Stanford Encyclopedia of Philosophy. Online: http://plato.stanford.edu/entries/physicalism/ [05.07.2021].

Stoljar, D. (2016). The semantic of "what it's like" and the nature of consciousness. Mind, 125, 1161-1198.

Stubenberg, L. (2016). Neutral Monism. In E. N. Zalta (Ed.), The Stanford Encyclopedia of Philosophy. Online: https://plato.stanford.edu/entries/neutral-monism/ [05.07.2021].

Swinburne, R. (1986). The evolution of the soul. Oxford: Oxford University Press. 
Swinburne, R. (2019). Are we bodies or souls? Oxford: Oxford University Press.

Tarski, A. (1936). Der Wahrheitsbegriff in den formalisierten Sprachen. Studia Philosophica, 1, 261-405.

Taylor, A. P. (2018). Review of "Idealism: new essays in metaphysics". Notre Dame Philosophical Reviews. Online: https://ndpr.nd.edu/news/idealism-new-essays-in-metaphysics/ [05.07.2021].

Taylor, J. M. \& Whalen, P. J. (2015). Neuroimaging and anxiety: the neural substrates of pathological and non-pathological anxiety. Current Psychiatry Reports, 17:49.

Tiehen, J. (2016). Physicalism requires functionalism: a new formulation and defense of the via negativa. Philosophy and Phenomenological Research, 93, 3-24.

Tiehen, J. (2018). Physicalism. Analysis, 78, 537-551.

Tononi, G., Boly, M., Massimini, M. \& Koch, C. (2016). Integrated information theory: from consciousness to its physical substrate. Nature Reviews Neuroscience, 17, 450-461.

Tye, M. (1995). Ten problems of consciousness. Cambridge: MIT Press.

Van Fraassen, B. (2002). The empirical stance. New Haven: Yale University Press.

Wallace, M. (2016). Saving mental fictionalism from cognitive collapse. Res Philosophica, 93, 1-20.

White, S. L. (1986). Curse of qualia. Synthese, 68, 333-368.

White, S. L. (2007). Property dualism, phenomenal concepts, and the semantic premise. In T. Alter \& S. Walter (Eds.), Phenomenal concepts and phenomenal knowledge: new essays on consciousness and physicalism (S. 210-248). Oxford: Oxford University Press.

Wilson, J. (2005). Supervenience-based characterizations of physicalism. Nous, 39, 426-459.

Wilson, J. (2006). On characterizing the physical. Philosophical Studies, 131, 61-99.

Wilson, J. (2010). What is Hume's dictum, and why believe it? Philosophy and Phenomenological Research, 80, 595-637.

Wilson, J. (2014). No work for a theory of grounding. Inquiry: An Interdisciplinary Journal of Philosophy, 57, 535-579.

Wilson, J. (2016). Grounding-based formulations of physicalism. Topoi, 37, 495-512.

Yih J., Beam, D. E. Fox, K. C. R. \& Parvizi, J. (2019). Intensity of affective experience is modulated by magnitude of intracranial electrical stimulation in human orbitofrontal, cingulate and insular cortex. Social Cognitive and Affective Neuroscience, 14, 339-351.

Zschocke, S. (2002). Klinische Elektroenzephalographie. Berlin: Springer. 